\title{
TIP60 acetylation of BMAL 1 links positive and negative arms of the molecular circadian clock
}

\author{
Dissertation \\ for the award of the degree \\ "Doctor rerum naturalium" (Dr. rer. Nat.) \\ of the Georg-August-Universität Göttingen \\ within the doctoral program Biology \\ of the Georg-August University School of Science (GAUSS) \\ submitted by \\ Nikolai Petkau \\ from \\ Jangiabad, Uzbekistan
}

Göttingen 2019 
Thesis Committee

Prof. Dr. Gregor Eichele, Genes and Behavior, Max-Planck-Institute for Biophysical Chemistry, Göttingen (Mentor and Reviewer)

Prof. Dr. Henning Urlaub, Bioanalytical Mass Spectrometry, Max-Planck-Institute for Biophysical Chemistry, Göttingen (Co-reviewer)

Members of the Extended Examination Board

Prof. Dr. Ahmed Mansouri, Molecular Developmental Genetics, Max-Planck-Institute for Biophysical Chemistry, Göttingen

Prof. Dr. André Fiala, Molecular Neurobiology of Behaviour, Schwann-Schleiden Research Centre, Göttingen

Prof. Dr. Detlef Doenecke, Molecular Biology, University Medical Center Göttingen

Dr. Alex Faesen, Biochemistry of Signal Dynamics, Max-Planck-Institute for Biophysical Chemistry, Göttingen

Date of oral examination: 04.12.2019 


\section{Affidavit}

I hereby declare that the dissertation entitled "TIP60 acetylation of BMAL1 links positive and negative arms of the molecular circadian clock" is submitted in support of my application for the degree of Doctor rerum naturalium to Georg-AugustUniversität Göttingen within the doctoral program Biology of the Georg-August University School of Science (GAUSS). This thesis has been written independently and with no sources and aids other than quoted within texts, references and acknowledgments. This thesis has not been submitted in any previous application for any degree, elsewhere.

Göttingen, October 2019

(Signature) 


\section{Table of Contents}

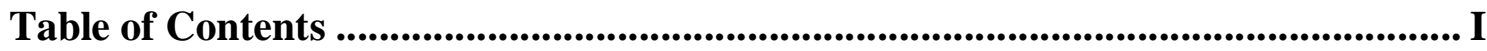

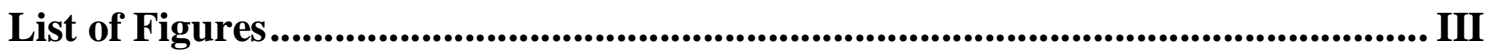

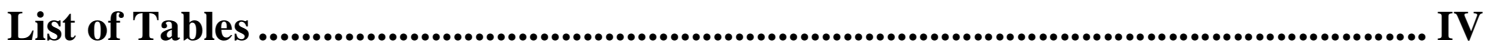

Summary V

Abbreviations n......................................................................................................... VII

Chapter 1: Introduction ..........................................................................................1

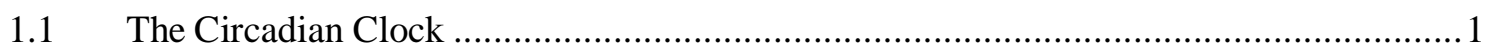

1.1.1 The Hierarchical Organization of the Mammalian Circadian Clock................................2

1.1.2 Molecular Organization of the Mammalian (Core) Circadian Clock ................................ 4

1.1.3 The Transcriptional Regulation of Circadian Genes ................................................. 10

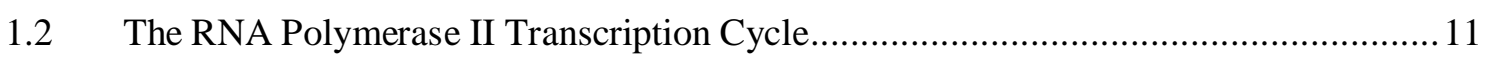

1.2.1 Transcription Initiation and Promoter Clearance ........................................................ 13

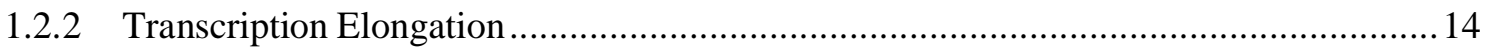

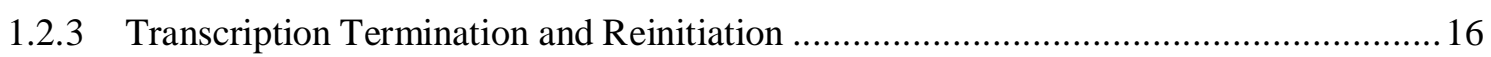

1.2.4 Role of TIP60 in Transcription Control ................................................................... 17

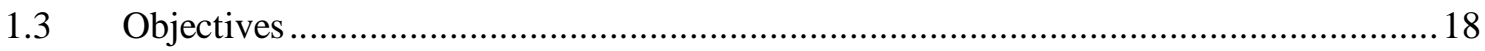

Chapter 2: Material and Methods ......................................................................20

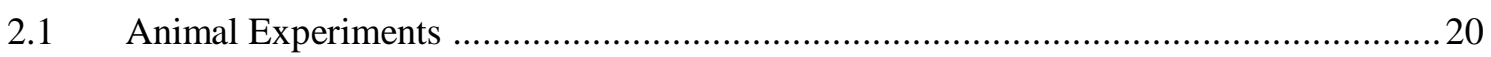

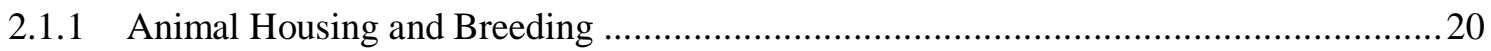

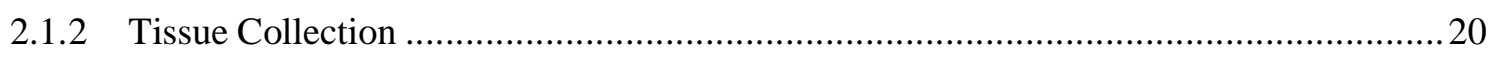

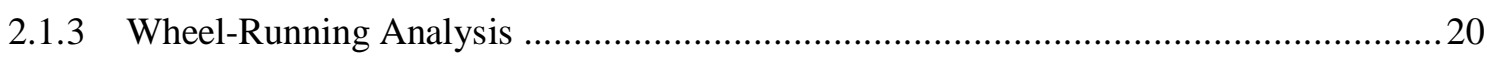

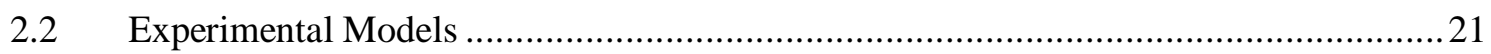

2.2.1 Tip60 Targeting Vector and Generation of Experimental Animals ...............................22

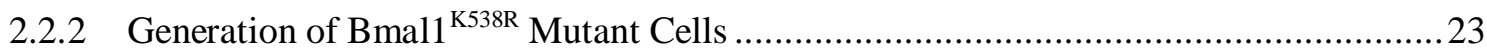

2.2.3 Generation of loxP-STOP-loxP-Tip60-3xFLAG BAC and Experimental Animals ........ 25

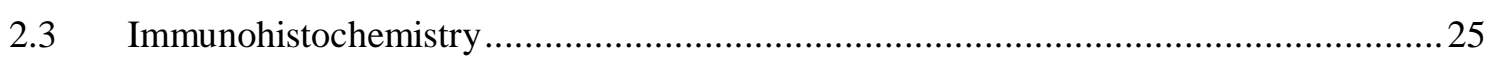

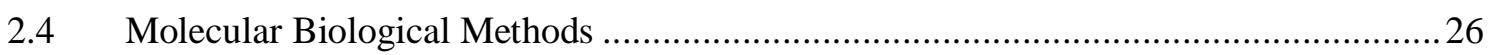

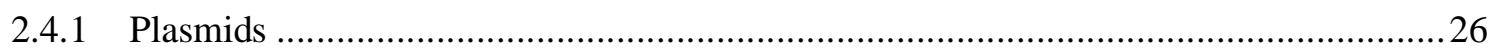

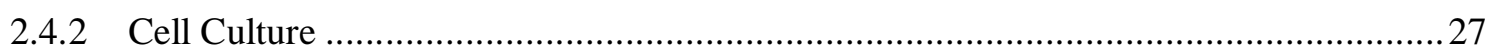

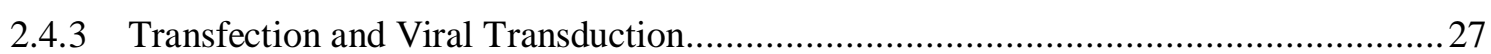

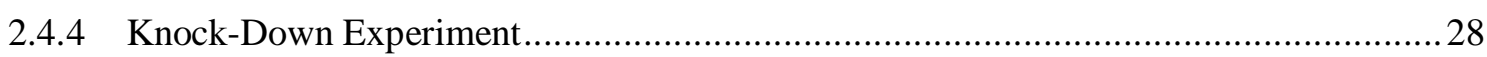

2.4.5 Preparation of Cell Extracts and Nuclear Extracts...................................................... 28

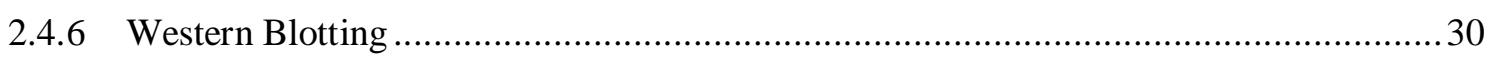

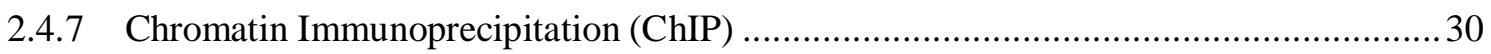


2.4.8 RNA Analysis by Quantitative Real-Time PCR (qPCR) ............................................. 34

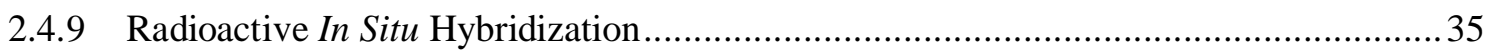

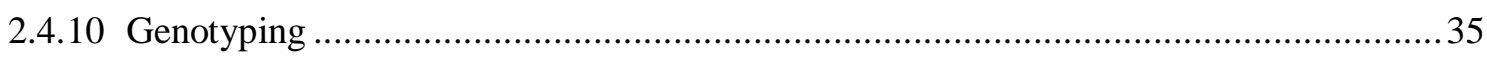

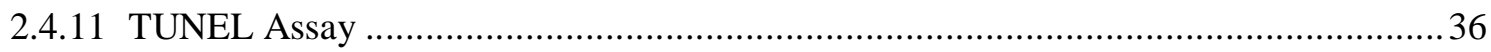

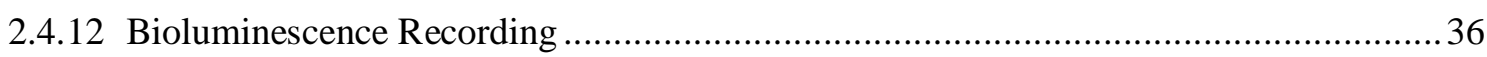

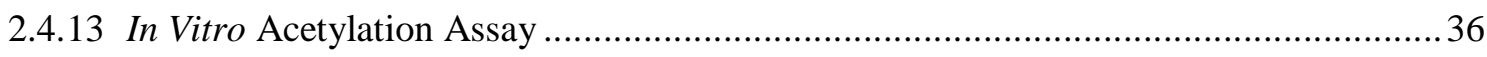

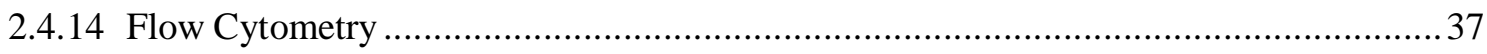

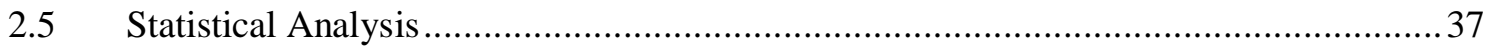

Chapter 3: Results ...............................................................................................................38

3.1 Inhibition of CDK9 and BRD4 Abolishes Circadian Oscillations..................................38

3.2 Acetylation of BMAL1 at Lysine 538 is Required to Initiate Circadian Productive

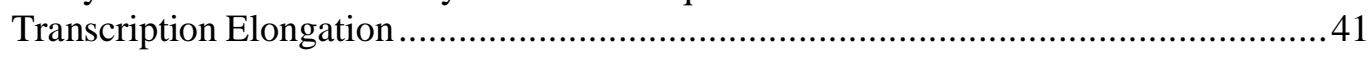

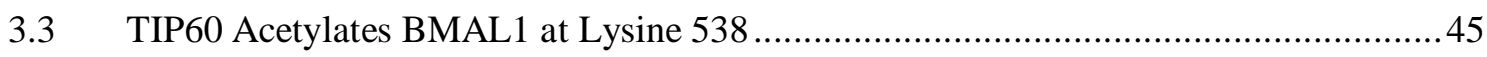

3.4 TIP60 Is Essential for a Functional Circadian Clock in the Mouse ...............................48

3.5 TIP60 Controls Productive Elongation of Circadian Transcripts .................................51

3.6 CRYs Oppose TIP60 and Repress Productive Elongation of Circadian Transcripts......53

3.7 Rhythmicity of Productive Elongation of Circadian Transcripts...................................58

Chapter 4: Discussion .....................................................................................61

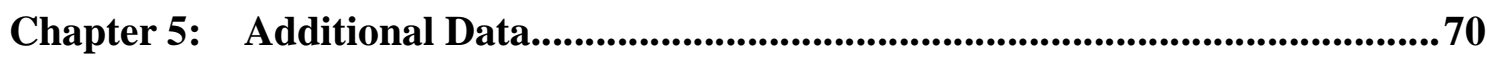

Chapter 6: References ....................................................................................71

Chapter 7: Publication .............................................................................................88

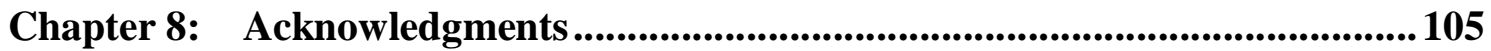




\section{List of Figures}

Figure 1: Representative Double-Plotted Actogram of a Mouse .................................................2

Figure 2: The Hierarchical Organization of the Mammalian Circadian Clock............................. 3

Figure 3: Molecular Organization of the Mammalian Circadian Clock.......................................5

Figure 4: The RNA Polymerase II Transcription Cycle .............................................................. 12

Figure 5: Transcription Regulation by the RNA Pol II CTD ................................................. 13

Figure 6: The Acetyltransferase TIP60 is Part of a Multiprotein Complex................................ 17

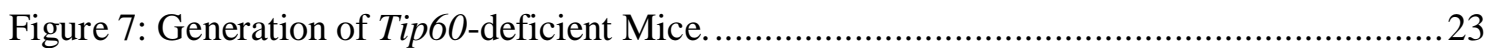

Figure 8: CRISPR/Cas9-mediated Generation of BMAL1 ${ }^{\text {K538R }}$ Mutant Cells. ...........................2 24

Figure 9: The loxP-STOP-loxP-Tip60-3xFLAG BAC Transgene. …........................................25

Figure 10: Validation of Antibodies used for ChIP Studies. ................................................... 33

Figure 11: RNA Pol II Pause Release Is a Regulatory Step of Clock Gene Expression.............. 38

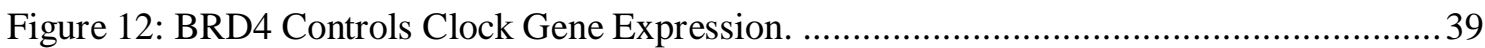

Figure 13: BRD4 Inhibition Does Not Directly Affect Bmal1 Expression.................................40

Figure 14: CRISPR/Cas9-mediated Generation and Characterization of BMAL1 ${ }^{\mathrm{K} 538 \mathrm{R}}$

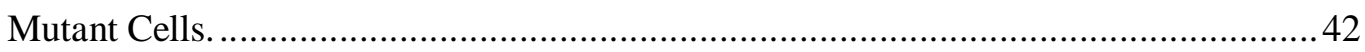

Figure 15: Lys538 Acetylation of BMAL1 Is Essential for Transcription Elongation. ...............43

Figure 16: Lys538 Acetylation of BMAL1 Controls Clock Gene Expression. ...........................44

Figure 17: Acetylation of BMAL1 Is Unlikely to Affect Transcription Initiation. ......................4 44

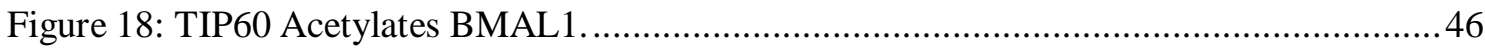

Figure 19: BMAL1 Is the Only Clock Protein Acetylated by TIP60...........................................47

Figure 20: TIP60-deficiency Evokes a Circadian Phenotype in Mice and Disrupts

Rhythmic Clock Gene Expression in the SCN...........................................................49

Figure 21: TIP60-deficiency Disrupts Rhythmic Clock Gene Expression in MEFs. ..................50

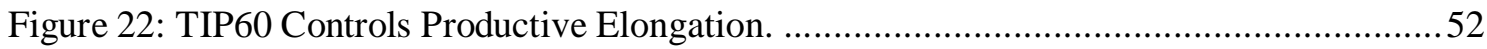

Figure 23: CRYs Compete with TIP60 for BMAL1 Binding. ....................................................54

Figure 24: CRYs Control Transcription Elongation.............................................................. 56

Figure 25: CRYs Control Lys538 Acetylation of BMAL1 in the Liver. ...................................57

Figure 26: Rhythmic Profile of Productive Elongation...............................................................59

Figure 27: Rhythmic Profile of BMAL1 Acetylation in the Liver. ........................................... 60

Figure 28: Model of RNA Pol II Pause Release Control at E-box-containing Clock Genes....... 62

Figure 29: Transcription factors can control initiation and/or RNA Pol II pause release. ...........64 


\section{List of Tables}

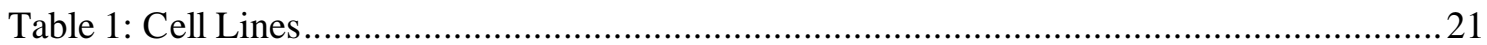

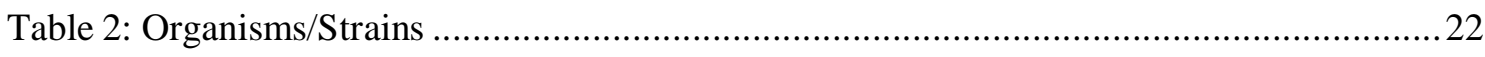

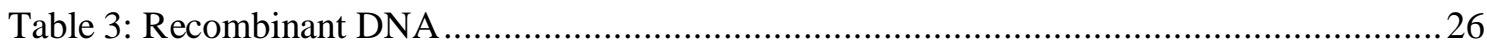

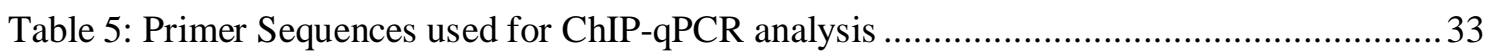

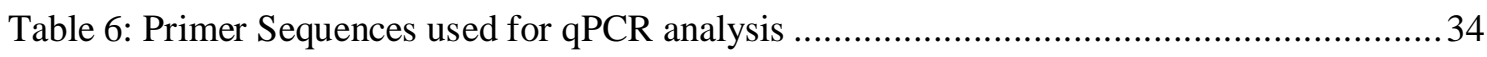

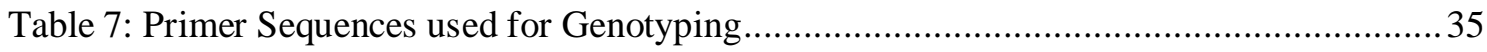

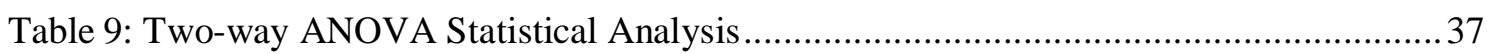




\section{Summary}

Many physiological processes exhibit circadian rhythms driven by cellular clocks composed of interlinked activating and repressing elements. The far-reaching influence of the circadian organization is illustrated by the fact that in each tissue $\sim 10 \%$ of the transcriptome is under the control of the circadian clock. Positive components are heterodimers of CLOCK and BMAL1 that bind to E-box promoter elements of Period and Cryptochome genes that encode repressor proteins CRY and PER. However, important issues that the transcriptional/translational feedback loop leave unresolved are (1) how binding of CLOCK-BMAL1 heterodimers to E-box promoter elements results in RNA Pol II-mediated transcription and (2) how exactly CRY proteins interfere with this process. Transcription is a highly complex process that is regulated at multiple levels. Well-recognized, rate-limiting step is the RNA Pol II release from promoter-proximal pause sites, which serves as a checkpoint, allowing rapid and synchronous expression of genes. This pause release of RNA Pol II and the transition to productive elongation requires the activity of the $\mathrm{P}-\mathrm{TEFb}$. $\mathrm{P}-\mathrm{TEFb}$ is recruited by interaction with specific factors such as BRD4 to gene promoters. BRD4 binds through its bromo-domains to acetylated lysines of histones and of transcription factors present at the promoters, thereby bringing P-TEFb to the site of action. Acetyltransferases that acetylate BRD4 binding partners play an important role in regulating RNA Pol II pause release. Of particular relevance for the present study is the lysine acetyltransferase TIP60. TIP60 is an essential protein and is involved in a multitude of cellular processes that depend on lysine acetylation of histone and non-histone proteins.

To investigate the temporal regulation of CLOCK-BMAL1-mediated transcription, mouse genetic approaches and analyses of interactions of key circadian proteins with each other and with E-box-containing clock gene promoters were combined. This approach provided evidence that the positive and negative phases of the circadian oscillator are linked through the acetyltransferase TIP60. The circadian clock cycle is initiated by BMAL1 binding to E-boxes of clock genes followed by a TIP60-mediated acetylation of chromatin bound BMAL1. This acetylation triggers recruitment of the BRD4-P-TEFb complex to E-box-containing circadian promoters leading to RNA Pol II pause release and productive elongation of circadian transcripts, including those encoding CRY and PER proteins. The study further shows that CRY repressors compete with TIP60 for BMAL1 to prevent TIP60 from acetylating BMAL1, thereby abolishing the binding of BRD4-P-TEFb complexes to circadian promoters and thus inhibiting productive 
transcript elongation. This competition characterizes the repressive limb of the clock cycle and eventually culminates in a complete cessation of BMAL1 acetylation and, thus, to the termination of clock gene transcription. Taken together, the experiments suggest that control of BRD4-P-TEFb recruitment to E-box-containing circadian promoters is a novel temporal checkpoint in the circadian clock cycle. 


\section{Abbreviations}

\begin{tabular}{|c|c|}
\hline AdCre & Adenovirus-expressing Cre recombinase \\
\hline AdGFP & Adenovirus-expressing green fluorescence protein \\
\hline ANOVA & analysis of variance \\
\hline $\mathrm{BAC}$ & Bacterial artificial chromosome \\
\hline BMAL1 & Brain and muscle ARNT-like protein 1 \\
\hline $\mathrm{bp}$ & base pair \\
\hline BRD4 & Bromodomain-containing protein 4 \\
\hline BSA & Bovine Serum Albumin \\
\hline CCGs & Clock-controlled output genes \\
\hline CDK9 & Cyclin-dependent kinase 9 \\
\hline ChIP & Chromatin immunoprecipitation \\
\hline CLOCK & Circadian locomotor output cycles kaput \\
\hline CMV & Cytomegalovirus \\
\hline Cre & Cre recombinase \\
\hline Cry & Cryptochrome \\
\hline $\mathrm{CT}$ & Circadian time \\
\hline DAPI & 4',6-diamidino-2-phenylindole \\
\hline Dbp & D-site albumin promoter binding protein \\
\hline DD & Constant darkness \\
\hline $\mathrm{ddH}_{2} \mathrm{O}$ & Double-distilled water \\
\hline ddNTP & Dideoxynucleotide triphosphate \\
\hline Dex & Dexamethasone \\
\hline DMEM & Dulbecco's Modified Eagle Medium \\
\hline DNA & Deoxyribonucleic acid \\
\hline DTT & Dithiothreitol \\
\hline ECL & Enhanced chemoluminescence \\
\hline EDTA & Ethylenediaminetetraacetic acid \\
\hline EGTA & Ethylene glycol tetraacetic acid \\
\hline et al. & et alii \\
\hline $\mathrm{EtOH}$ & Ethanol \\
\hline FBS & Fetal Bovine Serum \\
\hline FP & Flavopiridol \\
\hline HDAC & Histone deacetylase \\
\hline IP & Immunoprecipitation \\
\hline JQ1 & Triazolothienodiazepine \\
\hline $\mathrm{LD}$ & Light-dark cycle \\
\hline LoxP & locus of X-over P1 \\
\hline $\mathrm{P}-\mathrm{TEFb}$ & Positive transcription elongation factor $b$ \\
\hline PBS & Phosphate buffered saline \\
\hline PCR & Polymerase chain reaction \\
\hline
\end{tabular}


Per

qPCR

Rev-Erb

RNA Pol II

$\mathrm{SCN}$

SDS

SEM

Syt10

Tip60

ZT
Period

quantitative PCR

Reverse erythroblastosis virus

RNA Polymerase II

Suprachiasmatic nucleus

Sodium dodecyl sulfate

Standard error of the mean

Synaptotagmin 10

$60 \mathrm{kDa}$ Tat-interactive protein

Zeitgeber time 


\section{Chapter 1: Introduction}

\subsection{The Circadian Clock}

As a result of the Earth's rotation, living organisms developed an internal timing system called circadian clock that allows them to anticipate daily changes in their environment such as light and dark, food availability or temperature fluctuations (Pittendrigh, 1993). The term circadian derives from the Latin words circa (about) and diem (day) and describes the approximately 24-hour oscillation of biological processes. In mammals, the circadian oscillator controls daily rhythms of sleep and wakefulness, food and water consumption, and cognitive and physical performance (Beersma and Gordijn, 2007; Challet, 2019; Gerstner and Yin, 2010). Apart from behavior the circadian clock also regulates a variety of physiological parameters such as body temperature, hormone production and secretion, blood pressure, and numerous metabolic processes (Bass and Takahashi, 2010; Green et al., 2008; Panda, 2016).

Anticipating recurrent events is not the only function of the circadian oscillator. It provides additional important benefits for the organism, such as the temporal separation of chemically incompatible biological processes and the restriction of potentially harmful, but essential, chemical reactions to the times when they are required (Schibler et al., 2015).

A remarkable feature of the circadian clock is that it persists in the absence of rhythmic external time cues (zeitgebers) such as light or temperature. For example, mice kept under constant darkness show robust locomotor activity rhythms with a free-running period slightly shorter than 24-hours (Figure 1). This persistence of rhythmicity illustrates an internally driven and self-sustained timekeeping system. The discovery of the selfsustained nature of circadian rhythms is quite old and began with the study of the plant Mimosa pudica. In the 1700s, the French astronomer d'Ortous de Mairan observed diurnal leaf movements not only under standard light-dark conditions but also under constant darkness. Another important feature of the circadian system is that it is dynamic and can adapt to different external cues. For instance, the circadian clock can be synchronized, or entrained, by exogenous zeitgebers like light or temperature. This allows the organism to re-adjust to the new environment (Refinetti, 2015). 


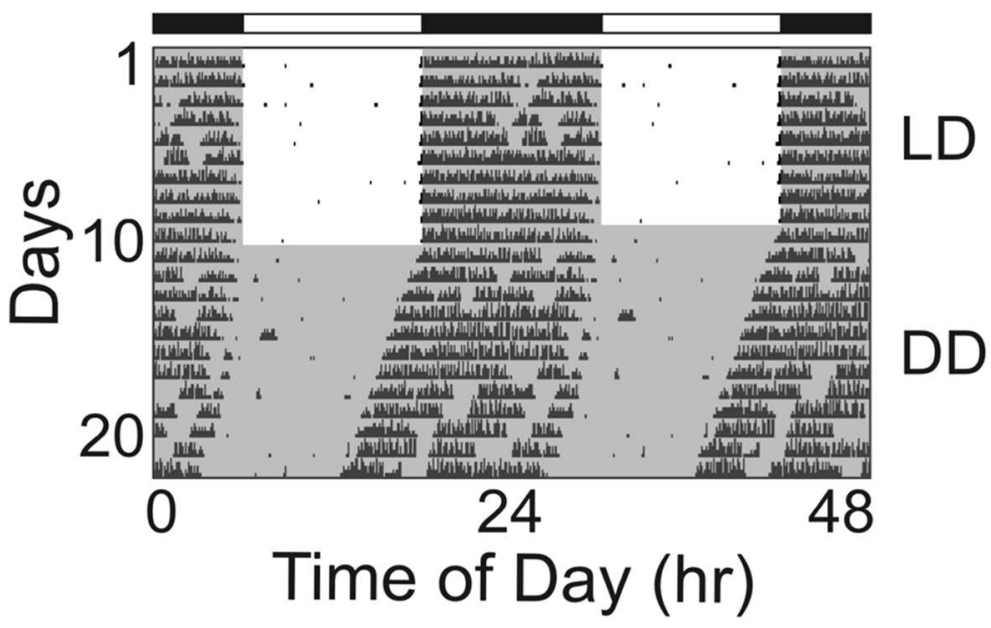

Figure 1: Representative Double-Plotted Actogram of a Mouse.

Wheel-running activity of a mouse is plotted as an actogram, where horizontal lines represent individual days and black vertical bars illustrate the activity (number of wheel revolutions). The mouse kept in constant darkness (DD) shows a robust free-running rhythm that is slightly shorter than 24-hours. The black and white bars at the top of the diagram show darkness (12-h) and light (12-h), respectively. For better visualization, the actogram is displayed twice by horizontally aligning two consecutive days. LD, lightdark cycle; DD, constant darkness

Disruption of the circadian clock either by mutations in key circadian genes or through misalignment between the internal clock and the environmental rhythms have a major impact on the well-being of the organism (Bedrosian et al., 2015; Musiek and Holtzman, 2016; Reid and Zee, 2009). Familial advanced sleep phase syndrome (FASPS) is an example of genetic mutation affecting the human molecular circadian machinery (Mendlewicz, 2009). Circadian desynchrony, a characteristic of shift work and sleep disruption in humans, can impair metabolic homeostasis resulting in insulin resistance and obesity. Furthermore, desynchrony of the circadian clock results in an increased risk of heart diseases, type 2 diabetes, and cancer (Verlande and Masri, 2019).

\subsubsection{The Hierarchical Organization of the Mammalian Circadian Clock}

In mammals, practically all cells harbor a cell-autonomous and self-sustained circadian oscillator with a similar molecular composition (see below). These numerous clocks are synchronized by a central pacemaker residing in the suprachiasmatic nucleus (SCN) of the ventral hypothalamus (Figure 2) (Dibner et al., 2010). The SCN, a bilateral structure of about 20,000 neurons located above the optic chiasma, receives light inputs from specialized photoreceptors of the retina through the retinohypothalamic tract and maintains phase coherence with the external world. 
A

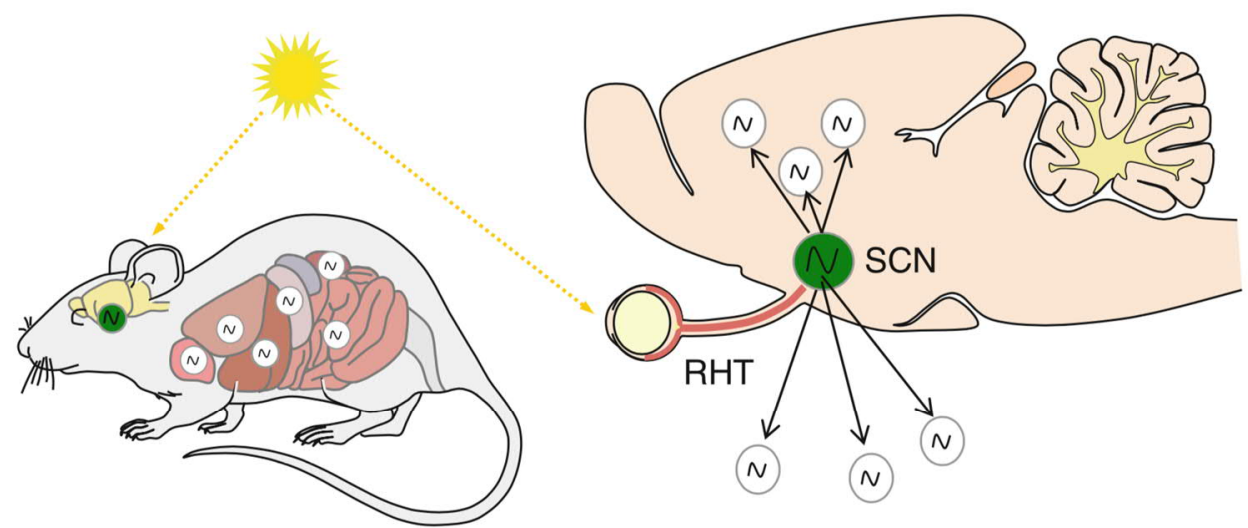

B

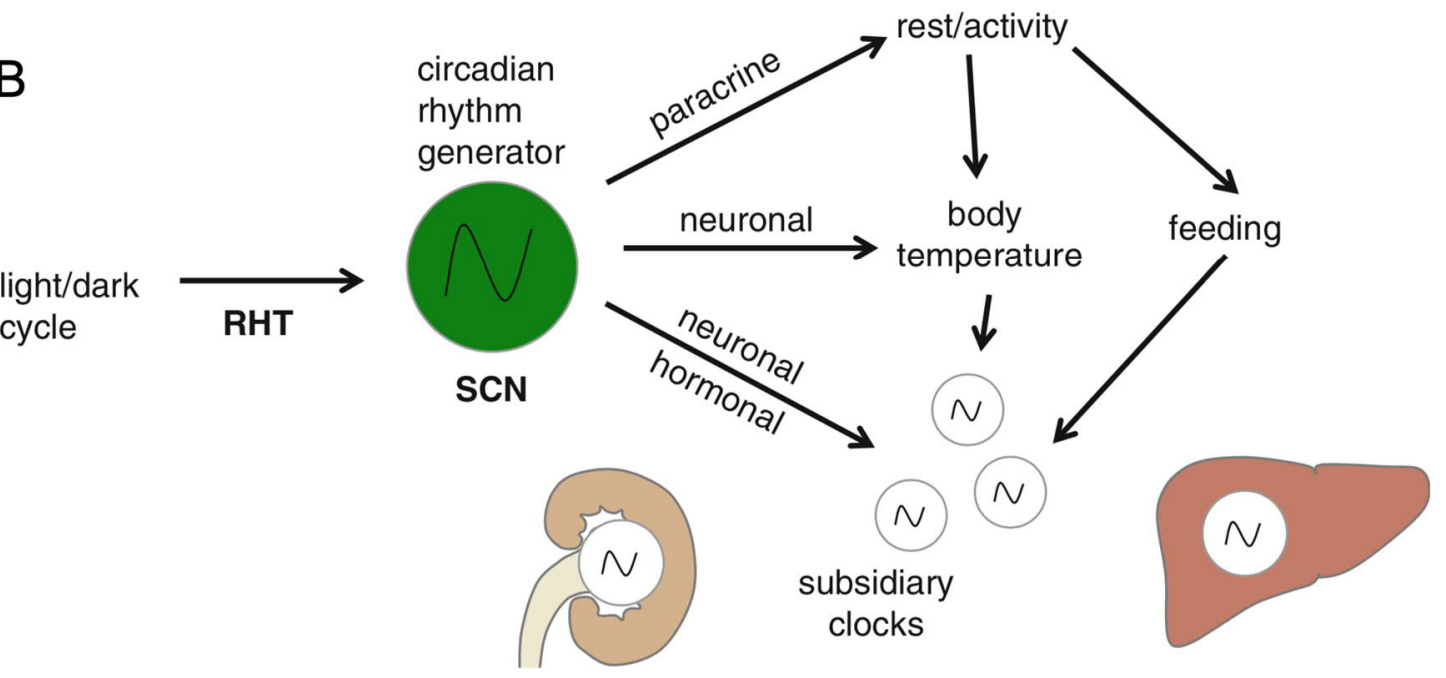

Figure 2: The Hierarchical Organization of the Mammalian Circadian Clock.

(A and B) The central pacemaker resides in the suprachiasmatic nucleus ( $\mathrm{SCN}$ ) and receives light inputs from specialized photoreceptors of the retina through the retinohypothalamic tract (RHT) and maintains phase coherence with the external world. This central pacemaker in turn synchronizes peripheral clocks located throughout the body and maintains rhythmic behavior and physiology at the organismic level. (B) Synchronicity is achieved through a multitude of signaling pathways. Neuronal and hormonal outputs are direct pathways of the SCN to coordinate peripheral clocks. The feeding-fasting rhythm on the other hand is indirectly driven through the sleep-wake cycle, that is controlled by paracrine signals of the central pacemaker. Feeding-fasting rhythms represent the most potent synchronization signals for peripheral organs, like the liver. Finally, body temperature rhythms are another important signal to synchronize peripheral clocks. These rhythms are generated by the SCN either directly through neuronal signals or indirectly through the control of the sleep-wake cycle. The illustration is adapted from Korf and von Gall (2013).

The SCN synchronizes the oscillators in peripheral tissues, on the one hand, through direct systemic cues such as hormones, metabolites, temperature or the sympathetic nervous system (Dibner et al., 2010). On the other hand, the SCN is able to synchronize peripheral tissues through indirect zeitgebers like feeding-fasting rhythms, that are driven 
by the SCN through the sleep-wake cycle (Dibner et al., 2010; Schibler et al., 2015). Feeding-fasting rhythms represent the most potent synchronization signals for circadian oscillators of peripheral organs, like the liver (Plautz et al., 1997; Schibler et al., 2015). The synchronization cues are apparently diverse in SCN and peripheral cells. Noteworthy, the SCN is mainly unresponsive to the synchronization signals it uses to entrain peripheral oscillators (Balsalobre et al., 2000; Buhr et al., 2010; Damiola et al., 2000).

Surgical ablation or genetic disruption of the circadian clock in the SCN leads to loss of phase coherence between the various peripheral tissues illustrating the essential role of the SCN for orchestration of consistent rhythms at the organism level (Husse et al., 2011; Moore and Eichler, 1972; Stephan and Zucker, 1972).

\subsubsection{Molecular Organization of the Mammalian (Core) Circadian Clock}

Although the central and peripheral oscillators show certain differences, the clocks in these tissues are based on two interlocked transcriptional-translational feedback loops (Figure 3). At the heart of these oscillators is the basic helix-loop-helix (bHLH) domaincontaining transcription factor brain and muscle ARNT-like protein 1 (BMAL1) (encoded by Aryl hydrocarbon receptor nuclear translocator-like protein 1 (Arntll). BMAL1 forms a heterodimer complex with either circadian locomotor output cycles kaput (CLOCK) or neuronal Per-Arnt-Sim (PAS) domain protein 2 (NPAS2), two bHLH domain-containing transcription factors (Gekakis et al., 1998; King et al., 1997; Wu and Rastinejad, 2017). Subsequently, the complexes bind to cis-regulatory elements harboring E-boxes of target genes such as Period (encoded by Per1, Per2, and Per3) and Cryptochrome (encoded by Cryl and Cry2) during the activation phase (Gekakis et al., 1998; Kume et al., 1999; Shearman et al., 2000). PER and CRY proteins form large heterocomplexes with over 30 additional polypeptide subunits (Aryal et al., 2017), translocate into the nucleus, and inhibit their own expression during the repression phase (Lee et al., 2001; Takahashi, 2017). As repression progresses, repressor complexes are ultimately degraded by the proteasome (Gallego and Virshup, 2007; Preußner and Heyd, 2016) and a new cycle of Per and Cry transcription can start. 


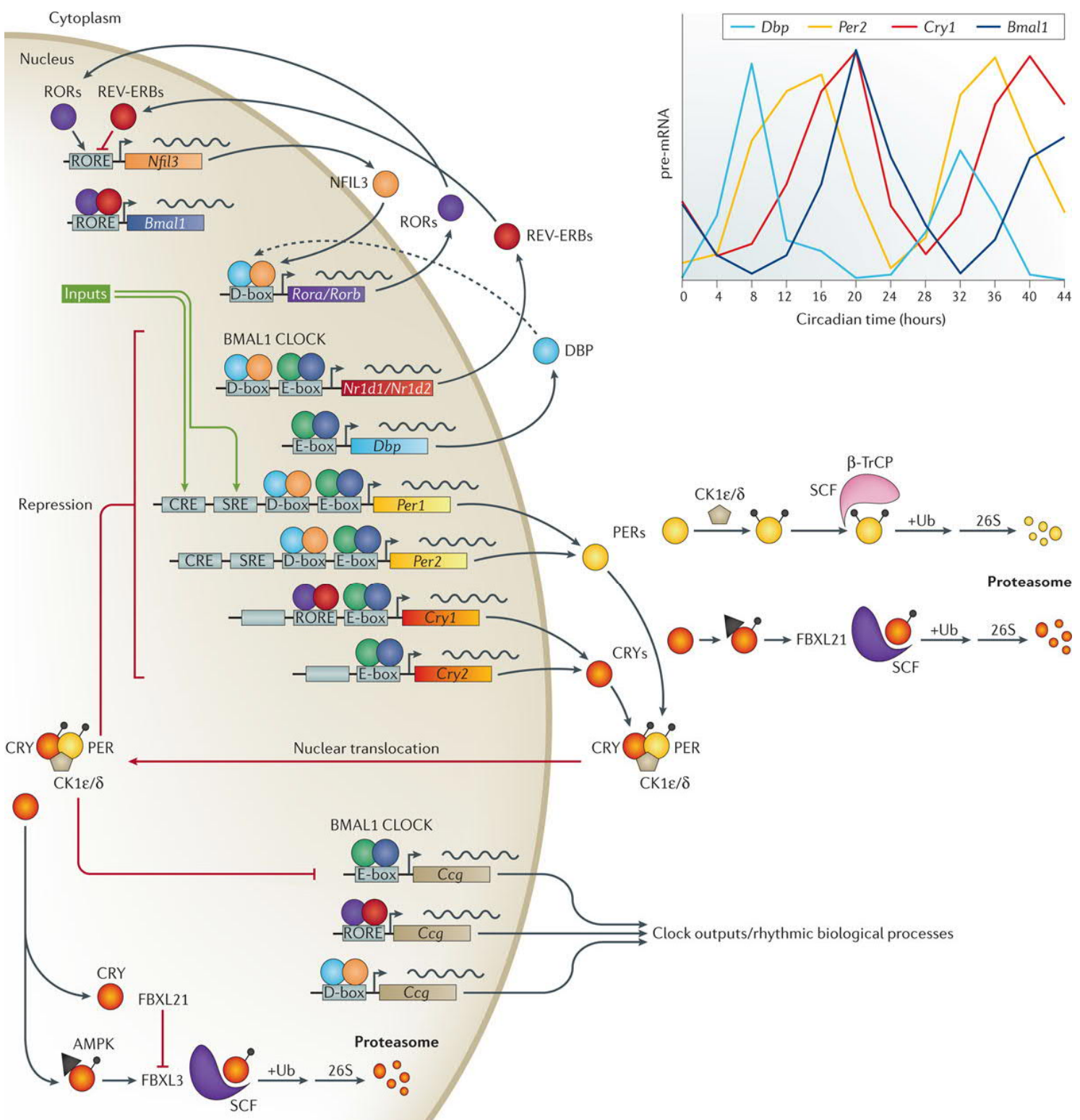

Figure 3: Molecular Organization of the Mammalian Circadian Clock.

At the heart of the circadian clock, transcription factors CLOCK and BMAL1 induce the expression of Per 1, Per2, Cryl and Cry2 genes. PER and CRY proteins translocate into the nucleus and repress their own transcription. Stability of the repressor proteins plays an important role in this scenario and is regulated by the ubiquitin-proteasome system. CLOCK-BMAL1 also induce the rhythmic expression of the nuclear receptors REV$\mathrm{ERB} \alpha$ and REV-ERB $\beta$, which in turn repress the transcription of Bmall and Nfil3 that are driven by the two activators $\operatorname{ROR} \alpha$ and $\operatorname{ROR} \beta$. The protein product of $N f i l 3$ itself is a repressor that counteracts the transcriptional activity of DBP and thereby drives the rhythmic expression of Ror genes. The interplay between these three interlocked transcriptional feedback loops drives the transcriptional output of the circadian clock. Various combinations of these loops generate different phases of rhythmic gene expression as illustrated by the graph (top right) showing the mRNA accumulation of different clock genes in the liver. The same principles apply to clock-controlled output genes (CCGs) as well where the regulatory regions of the target genes determine which 
of the three loops drives their rhythmic gene expression. AMPK, 5' AMP-activated protein kinase; CK1, casein kinase 1; CRE, cAMP response element; FBX, F-box protein; RORE, ROR-binding element; SCF, SKP1-cullin-F-box protein; SRE, serum response element; Ub, ubiquitin. The illustration is adapted from Takahashi (2017).

Recent genome-wide studies mapping the binding of core transcription factors and RNA Polymerase II (RNA Pol II) in the mouse liver revealed the presence of at least three distinct phases of the circadian cycle (Koike et al., 2012): (1) a transcriptionally active phase marked by the presence of the CLOCK-BMAL1 heterodimer along with the coactivators cAMP response element-binding protein (CREB)-binding protein (CBP) and histone acetyltransferase p300 (p300) (2) an early repression phase during which PER1, PER2, and CRY2 occupancy peaks and transcription declines and (3) a late repression phase characterized by a poised but transcriptionally silent, E-box-bound CLOCKBMAL1-CRY1 complex (Koike et al., 2012).

In addition to generating and maintaining self-sustained circadian rhythms, the circadian clock drives the rhythmic expression of thousands of clock-controlled output genes in various tissues. However, only a small proportion of these genes exhibit rhythmic expression in all tissues (Storch et al., 2002; Zhang et al., 2014). The circadian clock has rather the function to drive tissue-specific rhythmic expression of genes involved in various cellular processes such as metabolism (Bass and Takahashi, 2010; Green et al., 2008; Panda, 2016), immune function (Scheiermann et al., 2013), or cell proliferation (Gaucher et al., 2018). Transcriptome analysis of mice and a primate have shown that more than half and more than 80 percent of all genes cycle at the mRNA level in at least one tissue, respectively (Mure et al., 2018). Thereby, roughly 5-20 percent of genes expressed in any particular cell or tissue undergo circadian oscillation, thus the main task of the mammalian clock seems to be transcription control of clock target genes.

\subsubsection{The Positive Feedback Loop}

The CLOCK-BMAL1 heterodimer forms an $\sim 750 \mathrm{kDa}$ complex during the activation phase (Aryal et al., 2017) and is believed to orchestrate some of its role as transcription activator through recruitment of histone-modifying enzymes (DiTacchio et al., 2011; Katada and Sassone-Corsi, 2010; Menet et al., 2014; Siepka et al., 2007), opening up the chromatin allowing transcription to start. Involvement of additional transcription factors and epigenetic regulation was also supported by the observation that binding of the 
CLOCK-BMAL1 complex to its cognate DNA binding elements is insufficient to induce transcription (Etchegaray et al., 2003; Koike et al., 2012; Lee et al., 2001; Menet et al., 2012; Ripperger and Schibler, 2006; Stratmann et al., 2012).

At the beginning of the circadian transcription cycle, CLOCK-BMAL1 heterodimer directly interacts with the transcriptional coactivators p300 and CBP. This interaction is believed to result in acetylation of histones surrounding E-boxes providing an accessible chromatin state resulting in recruitment of the general transcription machinery (Curtis et al., 2004; Etchegaray et al., 2003; Hosoda et al., 2009; Koike et al., 2012; Lee et al., 2010; Takahata et al., 2000). CLOCK was also shown to harbor intrinsic acetyltransferase activity and to contribute to loci specific acetylation of histones (Doi et al., 2006). CLOCK, p300, and CBP share similar histone targets. Therefore, it was proposed that they could function at different phases of circadian transcription activation (Papazyan et al., 2016). CLOCK could acetylate and evict histones early in the activation phase and p300/CBP could interact with CLOCK-BMAL1 and acetylate neighboring histones later in activation, thereby further opening chromatin and stimulating transcription (Curtis et al., 2004; Doi et al., 2006; Lee et al., 2010; Menet et al., 2014; Papazyan et al., 2016). It was also reported that CLOCK could acetylate its own heterodimeric partner BMAL1 at lysine 538 (Lys538) (Hirayama et al., 2007). CLOCK-mediated acetylation of BMAL1 is supposed to take place during the repression phase and mediate CRY1 recruitment to BMAL1 (Hirayama et al., 2007). Another cofactor that interacts with CLOCK-BMAL1 and stimulates histone acetylation is lysine-specific demethylase 5A (KDM5A) (DiTacchio et al., 2011). KDM5A functions as an antagonist of histone deacetylase 1 (HDAC1), thereby enhance the acetylation of histones at clock gene promoters. CLOCKBMAL1 does not only recruit cofactors that are involved in histone acetylation but also recruits various isoforms of mixed-lineage leukemia (MLL) family of methyltransferases (Katada and Sassone-Corsi, 2010; Valekunja et al., 2013), thus contributing to transcription activation. An additional cofactor that is recruited by CLOCK-BMAL1 is thyroid hormone receptor-associated protein-150 (TRAP150) (Lande-Diner et al., 2013). TRAP150 was reported to link the general transcription machinery to circadian target gene promoters by association with mediator complex subunit 1 (MED1). CLOCKBMAL1 heterodimer itself was also shown to function as a pioneer transcription factor that can bind nucleosomes to facilitate rhythmic chromatin remodeling and incorporation of the histone variant H2A.Z (Menet et al., 2014). This rhythmic chromatin opening is believed to promote binding of the general transcription machinery and of additional 
transcription factors. Thus, the CLOCK-BMAL1 heterodimer is clearly involved in RNA polymerase II (RNA Pol II) recruitment and initiation (Koike et al., 2012; Le Martelot et al., 2012). However, further steps such as promoter proximal pausing, pause release, and productive elongation were proposed also to play a role in clock gene regulation (for details about transcription cycle, see below) (Takahashi, 2017; Westermark, 2016; Zhu et al., 2018).

\subsubsection{The Negative Feedback Loop}

Repressive complexes containing PER and CRY proteins are driving the negative feedback loop of the circadian clock. The stability and subcellular localization of these complexes are essential factors to maintain precision and set the 24-h period length of the circadian clock (Busino et al., 2007; Eide et al., 2005; Godinho et al., 2007; Hirano et al., 2013; Hirota et al., 2008; Isojima et al., 2009; Lamia et al., 2009; Lee et al., 2009; Partch et al., 2006; Reischl et al., 2007; Shirogane et al., 2005; Siepka et al., 2007; Yoo et al., 2013). Modulation of the balance between kinases, phosphatases, and ubiquitin E3 ligases that post-translationally modify the PER and CRY proteins, either by gene mutations or pharmacological inhibition, generates periods ranging from $\sim 20$ to 44 hours (Chen et al., 2012; Hirota et al., 2010; Toh et al., 2001; Xu et al., 2005). Thus, post-transcriptional regulation of the repressor complexes represents a key element of the negative feedback loop that helps to establish the essential delays in feedback regulation.

Genome-wide analysis of the core circadian regulators revealed a well-orchestrated chromatin association of circadian activators and repressors in the course of the circadian day (Koike et al., 2012; Menet et al., 2012; Rey et al., 2011). It was found that at the onset of the circadian cycle (late night/early morning) CRY1 is associated with chromatinbound CLOCK-BMAL1-RNA Pol II complexes without PER and is implicated in preventing E-box-mediated transcription. The existence of such 'late repressive complex' is supported by the observation that PER proteins are negligible for CRY1-mediated on DNA repression of CLOCK-BMAL1 transcriptional activation (Chiou et al., 2016; Ye et al., 2011, 2014) and is consistent with the unique ability of CRY1 to drive rhythms in minimal cellular oscillators (Khan et al., 2012; Liu et al., 2007; Ukai-Tadenuma et al., 2011). The prominent role of CRY1 is also reflected in the fact that CRY1 is more essential than CRY2 for rhythm generation and that CRY2 is a weaker repressor of the CLOCK-BMAL1 heterodimer (Rosensweig and Green, 2018). Upon degradation of chromatin-associated CRY1, coactivators are recruited followed by Per and Cry 
transcription. During the early repression phase of the circadian cycle (early evening), newly synthesized CRY2 and PER repressors associate with CLOCK-BMAL1, thereby shutting down E-box-mediated transcription. However, the mechanism of PER-mediated repression of the 'early repressive complex' seems to be different to that of CRY1 and relies on attenuation of CLOCK-BMAL1 binding to the DNA (Chiou et al., 2016; Ye et al., 2014). The role of PER proteins was proposed to be that of scaffolding proteins that assemble a stable and several megadaltons big repressive complex (Aryal et al., 2017; Kim et al., 2015; Rosensweig and Green, 2018). Biochemical analysis revealed that at least 25 additional protein components are part of this complex that are capable to give rise to transcriptional termination and epigenetic rearrangement at the transcription start sites of clock-controlled genes (Brown et al., 2005; Duong and Weitz, 2014; Duong et al., 2011; Kim et al., 2014; Padmanabhan et al., 2012). One of the components is the Mi2-nucleosome remodeling and deacetylase (NuRD) transcriptional co-repressor with both ATP-dependent chromatin remodeling and histone deacetylase activity (Kim et al., 2014). Other components are the transcriptional repressor complex SWI-independent-3 (SIN3)histone deacetylase (HDAC) and the histone methyltransferase complex heterochromatin protein 1 gamma (HP1 $\gamma$ )-suppressor of variegation 3-9 homolog (SUV39H) that contribute to chromatin condensation (Duong and Weitz, 2014; Duong et al., 2011). Apart from chromatin modifiers the repressive complex also recruits the helicases DEAD-box helicase 5 (DDX5), DExH-box helicase 9 (DHX9), and senataxin (SETX) to termination sites on clock-controlled genes, where they prevent transcriptional termination (Padmanabhan et al., 2012).

Not only the circadian activators and repressors show temporally restricted chromatin associations but also RNA Pol II illustrates a rhythmic abundance at promoters and gene bodies of rhythmically expressed genes (Koike et al., 2012; Le Martelot et al., 2012). Thus, RNA Pol II recruitment and initiation are obviously under circadian control. RNA Pol II pausing seems to be another step of the transcription cycle (for more information see below) that is controlled by the clock. Rhythmic accumulation of Ser5 phosphorylation of RNA Pol II during the late CRY1-containing repression phase indicates that RNA Pol II is recruited to circadian promoters but is transcriptionally inactive (Koike et al., 2012) and is waiting for an additional regulatory step to be released. Thus, binding of CRY1 to CLOCK-BMAL1 may prevent productive transcription. 


\subsubsection{The Transcriptional Regulation of Circadian Genes}

Besides the Per and Cry genes, the CLOCK-BMAL1 transcription factor complex also drives the expression of the reverse erythroblastosis virus- $\alpha,-\beta(\operatorname{Rev}-E r b \alpha, \operatorname{Rev}-\operatorname{Erb} \beta)$ genes (encoded by nuclear receptor subfamily 1, group D, member-1, -2 (Nrld1 and Nrld2)) (Preitner et al., 2002; Sato et al., 2006) (Figure 3). The protein products of these genes compete at retinoic acid receptor-related orphan receptor (ROR)-binding elements (ROREs) with the transcription activators $\operatorname{ROR} \alpha, \operatorname{ROR} \beta$, and $\operatorname{ROR} \gamma$ and thereby repress transcription of core lock genes, such as Bmal1, Npas2, and Clock (Guillaumond et al., 2005; Takeda et al., 2012). The inhibitory function of REV-ERB $\alpha$ and REV-ERB $\beta$ is not just accomplished by restricting the access of ROR activators to target gene promoters. In addition, REV-ERBs recruit nuclear receptor co-repressor 1 (NCoR) and histone deacetylase 3 (HDAC3) to target gene promoters and establish a repressive chromatin state for long-term repression (Feng et al., 2011). The CLOCK-BMAL1-driven diurnal expression of Rev-Erb $\alpha$ and $\operatorname{Rev}-E r b \beta$ is essential for the maintenance of robust circadian expression of this interlocked accessory loop (Papazyan et al., 2016). A third E-boxdriven transcriptional loop involves the proline- and acidic amino acid-rich basic leucine zipper (PAR-bZip) transcription factors such as D-site albumin promoter binding protein (DBP), thyrotroph embryonic factor (TEF), and hepatic leukemia factor (HLF) (Takahashi, 2017) (Figure 3). These proteins bind to D-box elements, present in the promoters of clock output genes, rather than the core clock genes and show high functional redundancy (Gachon et al., 2004). Transcriptional activity of these factors is repressed by nuclear factor, interleukin-3-regulated (NFIL3) also known as E4BP4, that is driven by the REV-ERB loop (Gachon et al., 2004; Mitsui et al., 2001). In summary, these three interlocked transcriptional feedback loops generate rhythmic expression with a wide range of phases (Ueda et al., 2005). Combination of the three cis-elements ( $E$ boxes, ROREs, and D-boxes) in the promoters of clock genes coordinate clock transcription factor binding and gene transcription (Figure 3). For example, Cryl expression, which shows delayed accumulation relative to other direct CLOCK-BMAL1 target genes, is regulated by both E-boxes and ROREs cis-regulatory elements (UkaiTadenuma et al., 2011) and might explain its time restricted chromatin abundance (see above).

Although the composition of the circadian oscillator is quite similar throughout the body, there are several interesting differences in how they function in the SCN and peripheral tissues, such as the liver. In the SCN, CLOCK and BMAL1 are absolutely essential for 
the rhythmic expression of the repressors Perl and Per2 (Bunger et al., 2000; Schibler et al., 2015). However, in mice livers Perl and Per2 still show rhythmic expression in the absence of CLOCK and BMAL1, as long as they receive direct or indirect systemic cues from the SCN (DeBruyne et al., 2006; Kornmann et al., 2007a, 2007b). Cry1 and Cry2 transcripts lose their rhythmic expression in the same livers of CLOCK-deficient animals but are highly upregulated, which is in contrast to the function of CLOCK as a transcriptional activator (DeBruyne et al., 2006). On the contrary, expression of the two CLOCK-BMAL1 target genes $D b p$ and Rev-Erbo, that drive the rhythmic output of the interlocked accessary loops, is as expected strongly downregulated in both CLOCK- and BMAL1-deficient livers (Bunger et al., 2000; Damiola et al., 2000; DeBruyne et al., 2006; Kornmann et al., 2007b). Thus, the main function of CLOCK-BMAL1 in peripheral tissues is likely to control rhythmic expression of the two interlocked accessory loops, rather than that of Per and Cry genes (Schibler et al., 2015). This is in contrast to the function of CLOCK-BMAL1 in the SCN, where the heterodimer strictly regulates Per and Cry gene transcription and thereby produces self-sustained cycles of clock gene expression. Thus, analysis of core clock gene transcription in a tissue context are rather complex, as expression can be orchestrated by both the local oscillator and systemic cues (Figure 2). Ex vivo experiments with cells and tissue performed under steady state conditions (in the absence of rhythmic systemic cues) allowed a direct and simplified investigation of CLOCK-BMAL1 dependent transcription control (DeBruyne et al., 2007; Kornmann et al., 2007b). In this context, the E-box controlled regulators of the accessary loops represent robust readouts of the transcriptional activity of the CLOCKBMAL1 heterodimer independent of the cell context.

\subsection{The RNA Polymerase II Transcription Cycle}

As in the case of the circadian oscillator, the precise interplay between positive and negative regulatory factors determines the overall rate of transcription by RNA Pol II. The regulation of the eukaryotic transcription cycle occurs at multiple steps, starting with initiation, followed by elongation, and subsequently termination with release of RNA Pol II and the nascent transcript from the DNA template (Figure 4) (Core and Adelman, 2019; Jonkers and Lis, 2015; Liu et al., 2015; Porrua and Libri, 2015; Sainsbury et al., 2015). 


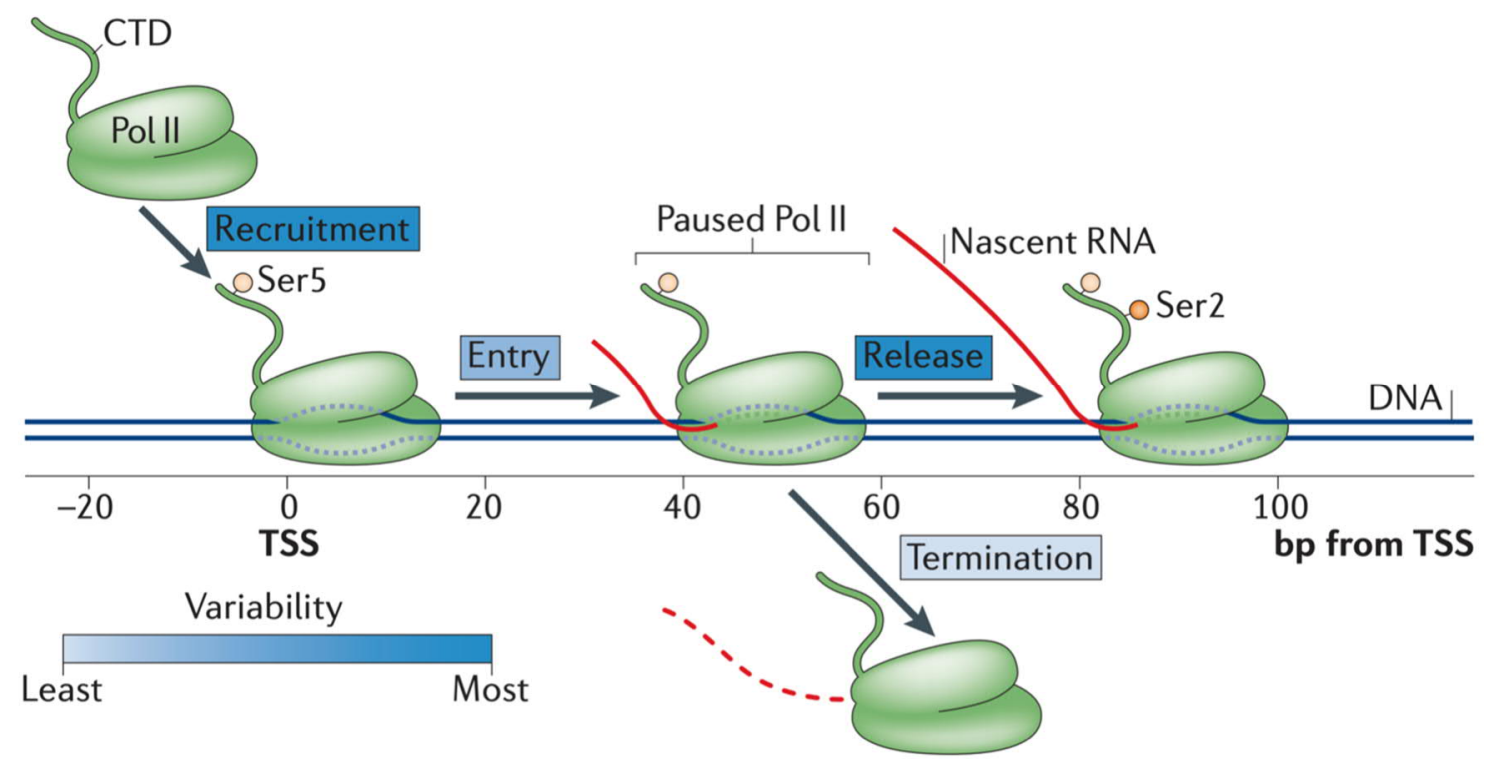

Figure 4: The RNA Polymerase II Transcription Cycle

Regulation of the transcription cycle at the transcription start sites (TSSs) takes place mainly at the level of promoter recruitment and release of RNA Pol II from promoterproximal pause sites. These two steps show the highest variability in terms of rate. Less variable and less regulated steps of the transcription are transcription entry to the pause site and transcription termination from the pause site. The illustration is adapted from Jonkers and Lis (2015).

The large subunit of RNA Pol II contains a carboxy-terminal domain (CTD) that is subjected to a large number of posttranslational modifications throughout the transcription cycle and acts as a binding platform for a multitude of transcriptionassociated factors involved in distinct stages of the transcription cycle. In addition, CTD serves as a scaffold for RNA maturation factors that process the RNA co-transcriptionally (Harlen and Churchman, 2017). The CTD of RNA Pol II is composed of 52 tandem heptapeptide repeats in mammals with a consensus sequence of Tyr1-Ser2-Pro3-Thr4Ser5-Pro6-Ser7 (Eick and Geyer, 2013; Harlen and Churchman, 2017). Phosphorylation of the serine residues at specific stages of the transcription cycle play an essential role in driving gene transcription, which will be discussed in more detail below (Figure 5). 


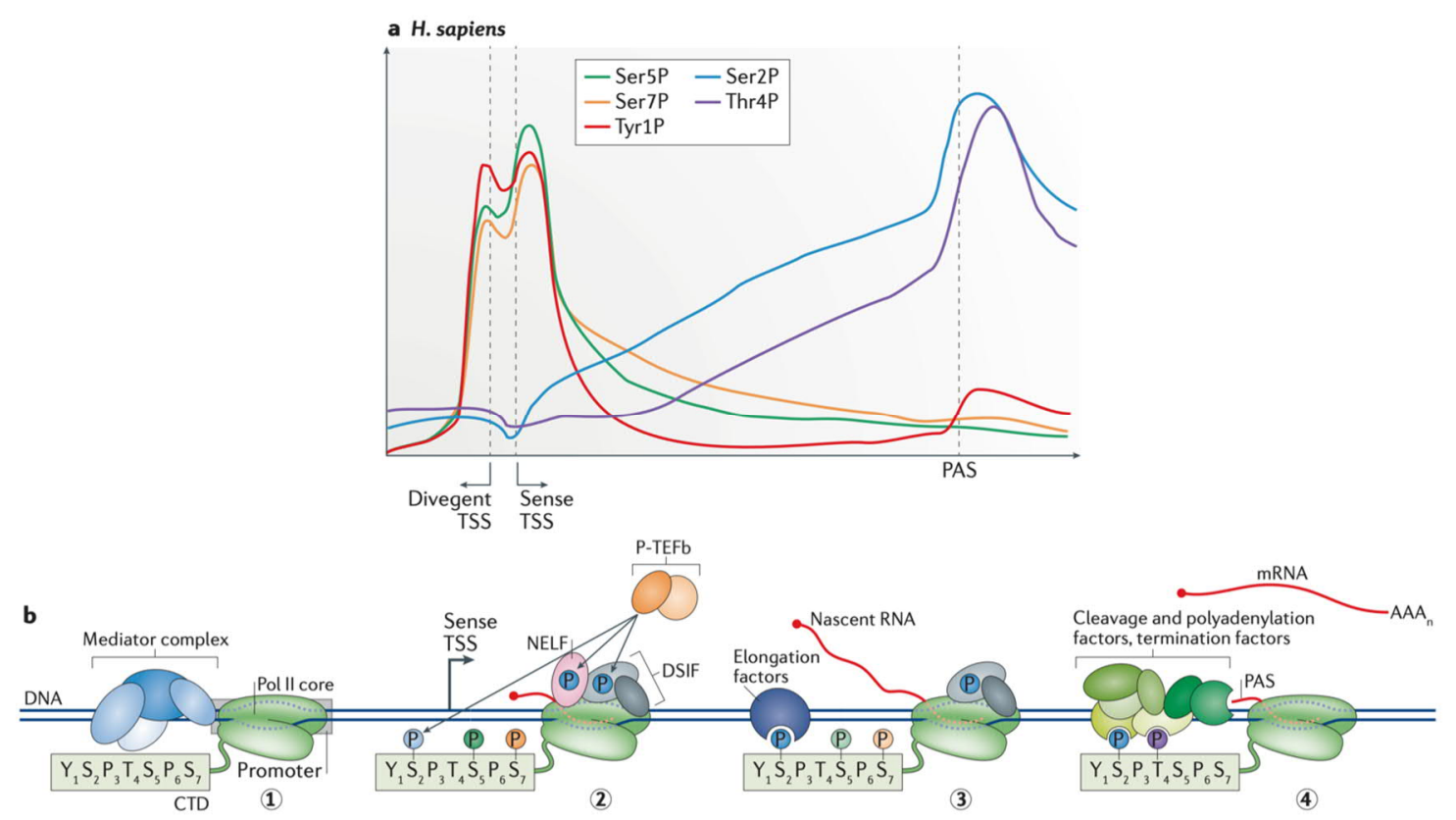

Figure 5: Transcription Regulation by the RNA Pol II CTD

(A) Chromatin occupancy profiles of phosphorylated residues of RNA Pol II CTD across protein-coding genes. (B) The distinct stages of the transcription cycle are accompanied by changes in the phosphorylation state of the CTD. (1) Hypoacetylated RNA Pol II is recruited to the promoter. After phosphorylation of Ser5 of the CTD by the CDK7 subunit of THIIH, Pol II escapes from the promoter. (2) RNA Pol II is paused downstream of TSSs, through interaction with NELF and DSIF, and is highly phosphorylated at Ser5 and Ser7 of the CTD. Recruitment of P-TEFb stimulates the transition into productive transcription elongation. P-TEFb phosphorylates NELF, DSIF and Ser2 of the CTD resulting in the release of NELF and productive transcription. (3) Phosphorylation of Ser2 promotes the recruitment of a multitude of transcription elongation, chromatinmodifying, and RNA-processing factors allowing co-transcriptional processes. As the RNA Pol II progresses toward the 3' end of the gene, dephosphorylation of Ser5 and Ser7 is catalyzed by phosphatases. (4) RNA Pol II reaches the polyadenylation site (PAS) and transitions from elongation to termination. Phosphorylation of Ser2 and Thr4 promotes the recruitment of cleavage, polyadenylation, and termination factors that release RNA Pol II from the DNA. The illustration is adapted from Harlen and Churchman (2017).

\subsubsection{Transcription Initiation and Promoter Clearance}

The first step of the transcription cycle is the formation of the preinitiation complex (PIC) at the promotor regions of genes. The stepwise PIC assembly starts with the binding of TFIID (transcription factor IID) or similar complexes to core promoter sequences. Subsequently, other general transcription factors (GTFs) like TFIIH (transcription factor IIH) and TFIIE (transcription factor IIE) together with the RNA Pol II are recruited to form the PIC. The ATP-dependent helicase activity of TFIIH leads to unwinding of the 
promoter DNA and formation of the transcriptionally competent open complex. This step provides access for RNA Pol II to the template strand and enables RNA synthesis. The emerging transcript enhances the stability of the transcribing complex and leads to promoter escape (Sainsbury et al., 2015).

Formation of PIC stimulates the kinase activity of the CDK7 subunit of THIIH, which then phosphorylates Ser5 and Ser7 of the CTD. Phosphorylation of Ser5 leads to the recruitment of RNA 5' capping enzymes that add a 7-methylguanosine $\left(\mathrm{m}^{7} \mathrm{G}\right)$ cap to the 5' end of nascent transcript. This protects the RNA from degradation by nuclear exoribonucleases and promotes their transport to the cytoplasm (Eick and Geyer, 2013; Harlen and Churchman, 2017) (Figure 5b-1).

\subsubsection{Transcription Elongation}

For a variety of genes, once RNA Pol II escapes the transcription start site (TSS), the elongating polymerase pauses 30-100 nucleotides downstream of the TSS and then experiences promoter-proximal pausing. Promoter-proximal pausing is believed to keep the promoter nucleosome-free and accessible to transcription factors (Gilchrist et al., 2010).

The pause release of RNA Pol II and the transition to productive elongation requires the activity of the positive transcription elongation factor $\mathrm{b}(\mathrm{P}-\mathrm{TEFb})$ complex, composed of T-type cyclins and cyclin-dependent kinase 9 (CDK9) (Jonkers and Lis, 2015; Liu et al., 2015). P-TEFb phosphorylates the pausing factors negative elongation factor (NELF) and DRB sensitivity inducing factor (DSIF). Upon phosphorylation, NELF is evicted from RNA Pol II and DSIF becomes a positive elongation factor. Accordingly, blocking efficient pause release by inhibition of CDK9 with flavopiridol augments promoterproximal pausing and downregulates global gene transcription (Jonkers et al., 2014; Rahl et al., 2010). Thus, promoter-proximal pausing seems to occur for nearly all RNA Pol IImediated transcription independent of whether paused RNA Pol II accumulates at the promoter-proximal sites or not. This checkpoint is believed to be important for the assembly of the elongation complex and for the recruitment of factors essential for elongation (Jonkers and Lis, 2015; Liu et al., 2015). Therefore, the release of RNA Pol II from promoter-proximal pausing sites serves as a well-recognized and rate-limiting step allowing for strong, rapid, and synchronous expression of genes (Boettiger and Levine, 2009; Gilchrist et al., 2012; Lagha et al., 2013; Lin et al., 2011; Liu et al., 2015). 
CDK9 also phosphorylates the CTD of RNA Pol II at Ser2 position and allows the transition to productive elongation. Phosphorylation of Ser2 promotes recruitment of transcription elongation factors, histone modifiers, chromatin remodelers, and the splicing machinery. As the RNA Pol II progresses toward the 3' end of the gene, dephosphorylation of Ser5 of the CTD is catalyzed by the phosphatase RNA Pol II subunit A C-terminal domain phosphatase (SSU72) (Eick and Geyer, 2013; Harlen and Churchman, 2017) (Figure 5b-2; 3).

\subsubsection{Recruitment of P-TEFb to Promoters}

$\mathrm{P}-\mathrm{TEFb}$ is rectuited to promoter-proximal sites through interaction with specific transcription factors and other proteins like bromodomain-containing protein 4 (BRD4) and super elongation complex (SEC) (Core and Adelman, 2019; Jonkers and Lis, 2015). BRD4 is a member of the BET (bromo-domain and extra terminal domain) protein family and harbors two amino-terminal bromodomains (BD1 and BD2), an extra-terminal domain and a carboxy-terminal P-TEFb interaction domain (PID) (Shi and Vakoc, 2014). Bromodomains are responsible to recognize acetylated lysine residues on histones and non-histone proteins. Complex formation between BRD4 and P-TEFb prevents P-TEFb from association with a ribonucleoprotein complex, 7SK/ hexamethylene bis-acetamide inducible protein 1 (HEXIM) that sequesters $\mathrm{P}-\mathrm{TEFb}$ in an inactive state (Core and Adelman, 2019; Jonkers and Lis, 2015).

Among the non-histone proteins, BRD4 was reported to interact with several specifically acetylated transcription factors. An example is the heterodimeric transcription factor complex twist family bHLH transcription factor 1 (TWIST1) - spermatogenic leucine zipper protein 1 (SPZ1) that uses BRD4 as a coactivator. Di-acetylation of SPZ1 at Lys369 and Lys374, and of TWIST1 at Lys73 and Lys76 leads to BRD4 recruitment and P-TEFb-mediated transcription activation (Shi et al., 2014; Wang et al., 2019). Another transcription factor whose acetylation facilitates BRD4 recruitment is the RELA/p65 subunit of nuclear factor kappa light chain gene enhancer in B cells (NF- $\kappa$ B). Lys310 acetylation of RELA/p65 leads to the recruitment of BRD4 and P-TEFb to NF- $\kappa \mathrm{B}$ target genes and their transcriptional activation (Huang et al., 2009; Zou et al., 2014).

Several small molecule inhibitors with high affinity towards BRD4 have been recently reported (Wang and Filippakopoulos, 2015). These inhibitors occupy the bromodomains and compete with acetylated lysine residues on histones and non-histone proteins. This, in turn, displaces BRD4 from chromatin and prevents its function in transcription 
activation. In accordance with this, treatment of cells with JQ1 disrupted the interaction between BRD4 and acetylated TWIST or acetylated RELA/p65, coinciding with the repression of TWIST- or NF-кB-induced transcriptional response (Shi et al., 2014; Zou et al., 2014).

Thus, BRD4 protein is a key player in the regulation of productive transcription elongation and is important for rapid and synchronous transcriptional induction, as observed in response to signal-dependent activation or during exit from mitosis (Shi and Vakoc, 2014). By implication, acetyltransferases that acetylate BRD4 binding partners would play an important role in regulating RNA Pol II pause release. Of particular relevance for the present study is the lysine acetyltransferase $60 \mathrm{kDa}$ Tat-interactive protein/K(lysine) acetyltransferase 5 (TIP60/KAT5). TIP60 is an essential protein (Hu et al., 2009), which is recruited to active promoters (Ravens et al., 2015) and was proposed to play a role in early steps of transcription elongation in mammals and flies (Kusch et al., 2004; Ravens et al., 2015; Shi et al., 2014; Wang et al., 2019).

\subsubsection{Transcription Termination and Reinitiation}

Once RNA Pol II transcribes the polyadenylation site, cleavage- and polyadenylation factors recognize this sequence element in the emerging transcript, and promote the cleavage and release of the nascent transcript. Ser2-phosphorylated CTD of RNA Pol II plays an essential role in this process and acts as a scaffold for the recruitment of these termination factors (Eick and Geyer, 2013; Porrua and Libri, 2015) (Figure 5b - 4).

The released RNA Pol II can enter subsequent a new round of transcription, however, it needs to go back to its original hypophosphorylated state. Several phosphatases were reported to catalyze the dephosphorylation of RNA Pol II and are essential for the recycling of RNA Pol II for another transcription cycle (Porrua and Libri, 2015; Shandilya and Roberts, 2012). Some of the general transcription factors remain bound with the promoter after RNA Pol II escapes from the PIC. This promoter associated GTFs act as a binding platform that allows reinitiation of RNA Pol II during successive rounds of transcription (Shandilya and Roberts, 2012). Factors associated with transcription termination also mediate formation of gene loops that brings the promoter bound GTFs and the 3'-ends of genes in close proximity and support efficient recycling and reinitition of RNA Pol II on the same DNA template (Hampsey et al., 2011). 


\subsubsection{Role of TIP60 in Transcription Control}

TIP60, is a member of the Moz, Ybf2/Sas3, Sas2, Tip60 (MYST) family of histone acetyltransferases and the catalytic subunit of the evolutionarily highly conserved nucleosome acetyltransferase of histone 4 (NuA4) complex (Jacquet et al., 2016). This complex, also known as TIP60 complex in mammals, is a large multiprotein complex consisting of at least 16 subunits (Figure 6B) (Doyon and Côté, 2004). Two distinct catalytic mechanisms have been proposed for TIP60 (Berndsen et al., 2007; Decker et al., 2008). The acetyltransferase either employs a ternary complex mechanism, where Glu403 serves as a general base and abstracts a proton from the substrate lysine (Berndsen et al., 2007), or a ping-pong mechanism via an acetyl-cysteine (Cys369) intermediate (Decker et al., 2008) (Figure 6B).
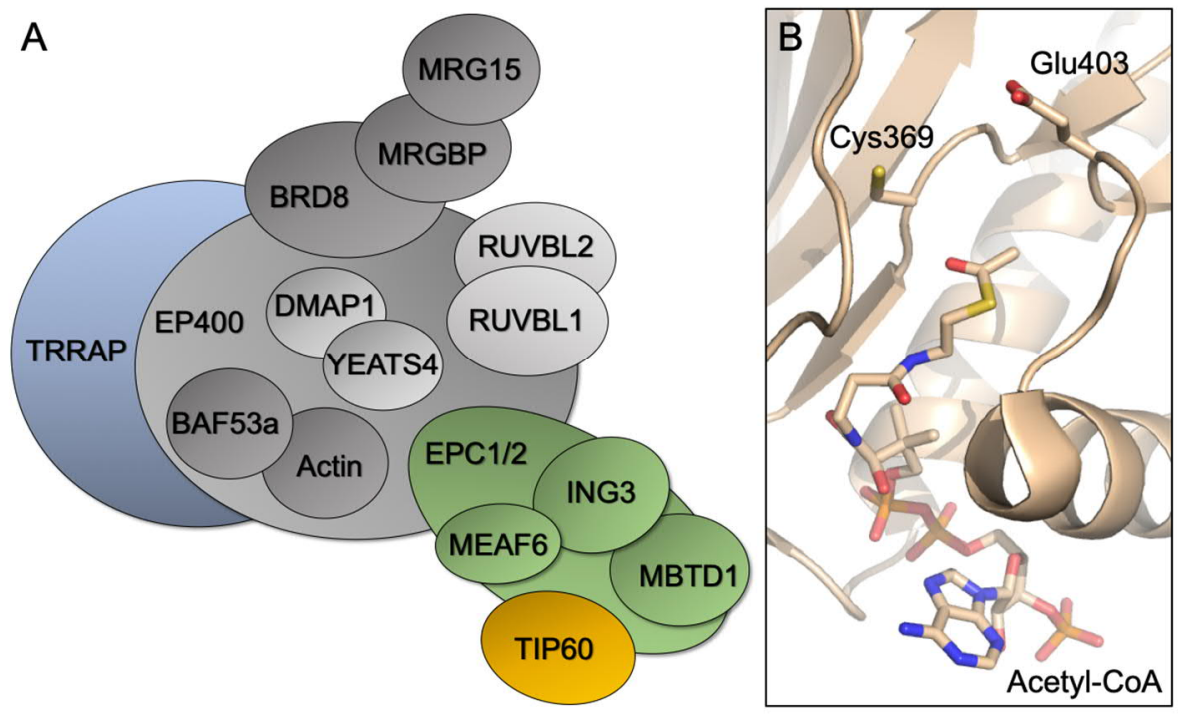

Figure 6: The Acetyltransferase TIP60 is Part of a Multiprotein Complex.

(A) Graphic showing the TIP60 complex composition. The illustration is adapted from Jacquet et al. (2016). (B) Close-up view of the active site of TIP60 co-crystallized with Acetyl-Coenzyme A (Acetyl-CoA). Catalytic residues are labeled. (PDB: 2OU2)

TIP60 is involved in a multitude of cellular processes such as DNA damage response, chromatin remodeling, apoptosis, and transcription regulation (Ghobashi and Kamel, 2018). In transcription, TIP60 acts as a coactivator through acetylation of histone and non-histone proteins and associates with a growing number of transcription factors (Ghobashi and Kamel, 2018; Judes et al., 2015). For example, TIP60 is known to acetylate the hinge region of androgen receptor (AR) augmenting its hormone-dependent transactivation potential (Clinckemalie et al., 2012). TIP60 also enhances transcriptional activity of NF- $\kappa$ B by physical interaction with the RELA/p65 subunit of NF- $\kappa B$ and maintaining Lys310 RELA/p65 acetylation (Kim et al., 2012). This leads to the 
recruitment of BRD4-P-TEFb and stimulates transcription of NF- $\kappa \mathrm{B}$ target genes (Huang et al., 2009; Zou et al., 2014). Another substrate of TIP60 is the transcription factor myelocytomatosis oncogene (c-MYC). Acetylation of c-MYC increases its protein stability (Patel et al., 2004). Furthermore, c-MYC recruits the TIP60 complex to c-MYC target genes, promoting histone $\mathrm{H} 4$ acetylation and target gene transcription (Frank et al., 2003). Similarly, E2F transcription factor 1 (E2F1) recruits the TIP60 complex on its target gene promoters in late $\mathrm{G} 1$ phase resulting in histone $\mathrm{H} 4$ acetylation and subsequent transcription (Taubert et al., 2004). Moreover, TIP60 coactivates the transcription factor $\mathrm{C} / \mathrm{EBP} \alpha(\mathrm{CCAAT} / \mathrm{enhancer}$ binding protein $\alpha)$ by physical interaction and acetylation of histones at $\mathrm{C} / \mathrm{EBP} \alpha$-target genes (Bararia et al., 2008). The best-studied transcription factor that is acetylated by TIP60 is tumor protein p53 (p53). TIP60 acetylates p53 at Lys120 within the DNA binding domain in response to DNA damage (Sykes et al., 2006; Tang et al., 2006). TIP60-mediated acetylation of p53 promotes expression of proapoptotic genes and initiates an apoptotic pathway to eliminate DNA-damaged cells (Charvet et al., 2011; Tang et al., 2006). Thus, TIP60 plays an important role in the decision between cell cycle arrest and apoptosis and maintains genome integrity. Two other transcription factors that are acetylated by TIP60 is TWIST1 and its heterodimeric partner SPZ1. TIP60 mediates di-acetylation of SPZ1 at Lys369 and Lys374, and of TWIST1 at Lys73 and Lys76, which are required for SPZ1-TWIST1 complex formation and BRD4 recruitment, thus enhancing RNA Pol II dependent transcription (Shi et al., 2014; Wang et al., 2019).

\subsection{Objectives}

Many physiological processes exhibit circadian rhythms driven by cellular clocks composed of interlinked activating and repressing elements. The far-reaching influence of circadian rhythmicity is highlighted by the fact that in each tissue $\sim 10 \%$ of the transcriptome is under the control of the circadian clock (Zhang et al., 2014) and that disruption of circadian rhythms is tied to the development of various diseases including metabolic disorders and cancer (Bedrosian et al., 2015; Musiek and Holtzman, 2016). Although significant progress was made in understanding the molecular principles of this oscillator and the field was even awarded with the Nobel Prize, still many open questions remain. One of them is: How does the CLOCK-BMAL1 heterodimer recruit the transcriptional machinery and thereby activate rhythmic gene transcription. Binding of 
CLOCK-BMAL1 to E-box elements alone is insufficient to induce transcription (Etchegaray et al., 2003; Koike et al., 2012; Lee et al., 2001; Menet et al., 2012; Ripperger and Schibler, 2006; Stratmann et al., 2012). Thus, additional factors are involved in the process of transcriptional activation of clock-controlled genes. In order to shed some light on this issue, a variety of different biochemical approaches are applied to identify several new components that regulate this process. In the center is a posttranslational modification of one of the heterodimeric partners that links CLOCK-BMAL1 with the transcriptional machinery. Subsequently, behavior analysis of mice that lack the enzyme mediating the posttranslational modification are presented to support the proposed model of transcriptional activation of E-box controlled clock genes.

Although CRY proteins were identified as key negative regulators of the mammalian circadian clock almost two decades ago (van der Horst et al., 1999), the mechanism by which the repressors fulfill their function is still not known. To fill up this gap, the repressive function of CRY-proteins is examined in the context of the new proposed model of transcriptional activation. To test this hypothesis interactions of CLOCKBMAL1 with the new identified components at clock gene promoters using CRY gainof-function and CRY loss-of-function cell models are presented. Finally, to converge the positive and negative limb of the circadian cycle changes in the interaction of the different components between each other and with clock gene promoters are presented over a course of 24-hours. 


\section{Chapter 2: Material and Methods}

Parts of this thesis have been published in:

Petkau N, Budak H, Zhou X, Oster H, Eichele G. 2019. Acetylation of BMAL1 by

TIP60 controls BRD4-P-TEFb recruitment to circadian promoters. Elife $\mathbf{8}$.

\subsection{Animal Experiments}

\subsubsection{Animal Housing and Breeding}

Animal experiments were carried out in compliance with the German Animal Welfare act (Tierschutzgesetz) and ethically approved and licensed by the Office for Consumer Protection and Food Safety of the State of Lower Saxony (Niedersächsisches Landesamt für Verbraucherschutz und Lebensmittelsicherheit). Mice were kept in transparent, individually ventilated cages under a 12 hours light and 12 hours dark (12:12 LD) cycle in a temperature- and humidity-controlled room at $21{ }^{\circ} \mathrm{C}$ and $55 \%$, respectively. Mice were fed standard chow ad libitum with free access to water.

\subsubsection{Tissue Collection}

Tissue collection was performed under constant darkness conditions (DD). Single-housed male mice were entrained to $12: 12 \mathrm{LD}$ cycle for at least 10 days and released into DD. For radioactive in situ hybridization analysis, mice were sacrificed at four time points on the second day in DD and brains were collected and frozen in O.C.T. medium (TissueTek). Tissue collection was mainly conducted by Dr. Harun Budak. For immunohistochemistry and TUNEL analysis brains were collected 42 hours after release into darkness and frozen in O.C.T. medium. Liver samples were collected at 6-hour intervals on the second day in DD and flash frozen in liquid nitrogen and stored at $-80^{\circ} \mathrm{C}$.

\subsubsection{Wheel-Running Analysis}

Wheel-running experiments were conducted as described previously (Jud et al., 2005). Male mice at the age of 2 to 6 months were single housed in cages equipped with a running 
wheel and revolutions of the wheel were detected via a magnetic switch connected to a computer system. Lighting conditions in the cages were adjusted by an external control device. Standard chow and water were provided ad libitum throughout the duration of the experiment. Wheel-running experiments were predominantly performed by Dr. Harun Budak.

Mice were entrained to an 12:12 LD cycle for at least 2 weeks and released into constant darkness. Free-running period $(\tau)$ of locomotor activity was calculated using $\chi 2-$ periodogram analysis by the ClockLab analysis software plug-in (Actimetrics) for MatLab (MathWorks).

\subsection{Experimental Models}

Cell lines and organisms used as experimental models in this study are listed in Table 1 and Table 2, respectively.

Table 1: Cell Lines

\begin{tabular}{|c|c|}
\hline Resource & Source \\
\hline NIH 3T3 Bmall-LUC & (Nagoshi et al., 2004) \\
\hline NIH 3 T3 Bmall-LUC; Bmal1 ${ }^{K 538 R}$ & This study \\
\hline Tip60fl/- MEFs & This study \\
\hline Tip60 fl/- Bmal1-LUC MEFs & This study \\
\hline Tip60 fl/- Bmal1-LUC; Tip60-V5 MEFs & This study \\
\hline Tip60 ${ }^{f l /-}$ Bmal1-LUC; Tip60 ${ }^{\mathrm{C} 369 \mathrm{~A} ; \mathrm{E} 403 \mathrm{Q}_{-} \text {V5 }}$ MEFs & This study \\
\hline Tip60 ${ }^{-1 /-}$ Bmall-LUC; Tip60-V5 MEFs & This study \\
\hline Cry $1^{+/+} ;$Cry $^{+/+}$fibroblasts & (Gauger and Sancar, 2005) \\
\hline Cry $1^{-/}$; Cry $2^{-/}$fibroblasts & (Gauger and Sancar, 2005) \\
\hline Clock $^{+/+}$MEFs & (Spengler et al., 2009) \\
\hline Clock $^{-/-}$MEFs & (Spengler et al., 2009) \\
\hline Bmall $^{+/+}$MEFs & (Spengler et al., 2009) \\
\hline Bmal1 $^{-/-}$MEFs & (Spengler et al., 2009) \\
\hline HEK239T & ATCC - CRL-3216 \\
\hline
\end{tabular}


Table 2: Organisms/Strains

\begin{tabular}{|c|c|}
\hline Resource & Source \\
\hline Rosa26 Flpe/Flpe: Gt(ROSA)26Sor ${ }^{\text {tm1(FLP1)Dym }}$ & JAX: 003946 \\
\hline CMV-Cre: $\operatorname{Tg}(\mathrm{CMV}$-cre $) 1 \mathrm{Cgn}$ & JAX: 006054 \\
\hline Syt10 $\mathrm{Cre/Cre}$ & (Husse et al., 2011) \\
\hline Syt10 $\mathrm{Cre/+}$ & (Husse et al., 2011) \\
\hline Tip60 flffl & This study \\
\hline Tip $60^{f / 1-}$ & This study \\
\hline Tip $60^{+/-}$ & This study \\
\hline Syt10 Crel+ Tip60 fll- & This study \\
\hline Syt10 Cre/Cre Tip60 fll- & This study \\
\hline LSL-Tip60-FLAG: Tg(loxP-STOP-loxP-Tip60-3xFLAG) & This study \\
\hline Tip60 fl/fl LSL-Tip60-FLAG & This study \\
\hline AlbCre: Speer6-ps1 $1^{\text {Tg(Alb-cre)21Mgn }}$ & JAX: 003574 \\
\hline Tip60 fllfl LSL-Tip60-FLAG AlbCre & This study \\
\hline Tip60 $0^{f l f l}$ AlbCre & This study \\
\hline
\end{tabular}

\subsubsection{Tip60 Targeting Vector and Generation of Experimental Animals}

The Tip60 flfl mouse line was generated by Dr. Xunlei Zhou using the following procedure: Bacteria (Strain EL350) and plasmids (pL451 and pL452) used for recombineering were obtained from the NCI (Biological Resources Branch). A 15.7-kb fragment of Tip60 genomic DNA was cloned from a BAC clone (bMQ-331N14, Sanger Institute) into a targeting vector carrying a Pol2-DTA cassette for negative selection. The first loxP site was inserted 637 bp upstream of the Tip60 ATG. The second loxP site together with a $F R T$-flanked $P G K$-neo cassette was inserted 3,065 bp downstream of the ATG. The 5' end of the homologous arm of the targeting vector was $4.5 \mathrm{~kb}$ and the 3' end of the homologous arm was $7.6 \mathrm{~kb}$. ES cell targeting and generation of Tip $60^{f / /+}$ founder mice was commissioned to PolyGene Transgenics (Switzerland). The FRTflanked PGK-neo selection cassette was removed by crossing mice with a Flippaseexpressing deleter line (Henrich et al., 2000). Targeting strategy for the Tip60 locus is provided in Figure 7. 
A $\begin{array}{lllllllll}1 & 2 & 3 & 4 & 5 & 6 & 7 & 8 & 9\end{array}$

1011

$1213 \quad 14$

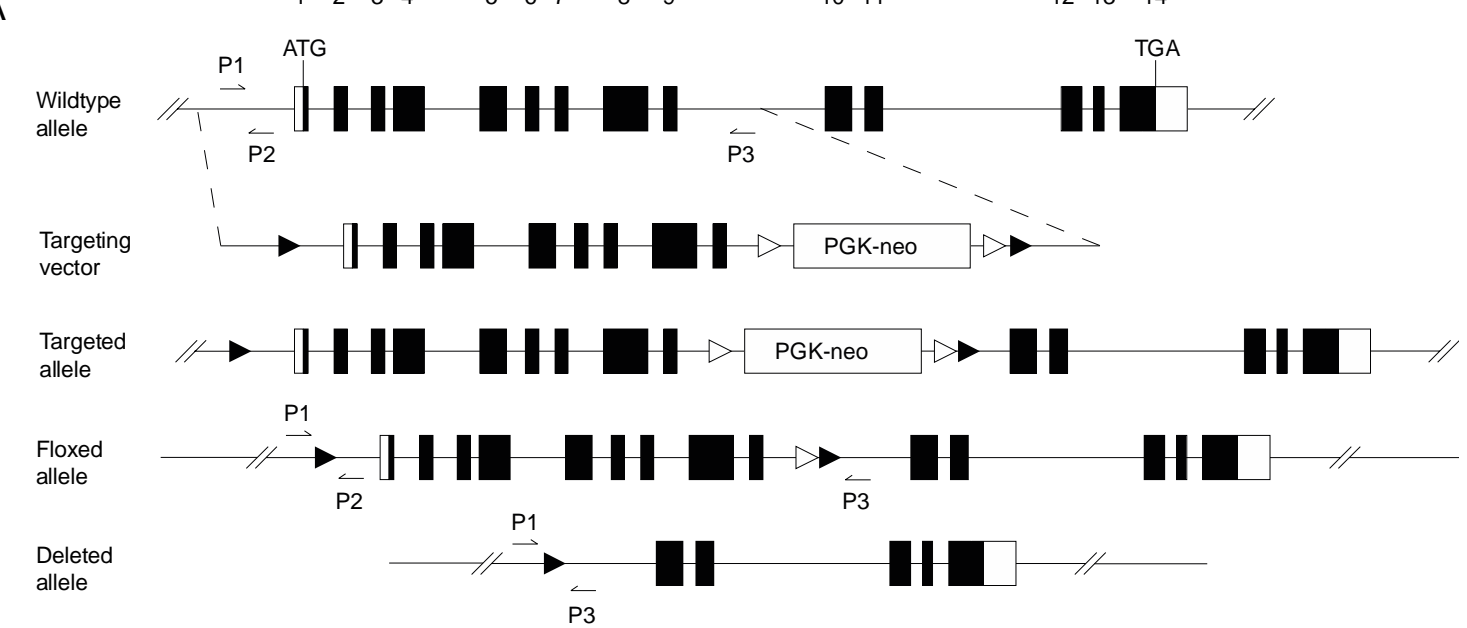

B

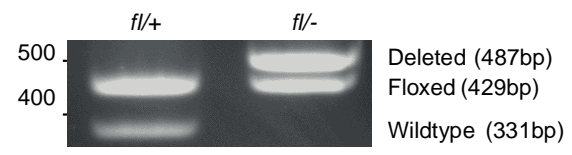

Figure 7: Generation of Tip60-deficient Mice.

(A) Targeting strategy for the Tip60 locus. Tip60 wildtype allele, targeting vector, targeted allele, floxed allele after removal of the PGK-Neo cassette, and the mutant Tip60 allele created by Cre-mediated recombination are shown. LoxP (black triangle) and FRT (open triangle) sites are marked. The arrows indicate the position of diagnostic primers P1-P3 (see Table 7). (B) PCR genotyping of Tip60 alleles. The wildtype allele produces a 331-bp amplicon (primers P1, P2) while the floxed allele produces a 429-bp amplicon (P1, P2). Deletion of the LoxP-flanked region of the Tip60 locus leads to a 487-bp fragment (P1, P3).

Mice deficient for TIP60 in the SCN were generated by Dr. Harun Budak and Dr. Xunlei Zhou. To generate these animals for locomotor activity recording, Tip60 flfl mice were crossed to a Syt10Cre driver mouse line resulting in Cre-mediated deletion of TIP60 predominately in the SCN (Husse et al., 2011). To generate Tip6 $60^{f /-}$ mice for subsequent MEF isolation, Tip60 flffl mice were crossed to a ubiquitous CMVCre driver mouse line (Schwenk et al., 1995) to generate Tip60 ${ }^{+/}$heterozygotes which were then used for breeding with Tip60 fl/fl mice to obtain Tip6 $0^{f l-}$ offspring. To generate animals for biochemical analysis, Tip $60^{f l f l}$ mice were crossed to a AlbCre driver mouse line resulting in TIP60 deletion exclusively in the liver (Postic et al., 1999). For all types of studies, mice were previously backcrossed to a C57BL/6 background for at least 10 generations.

\subsubsection{Generation of Bmal1 ${ }^{\mathrm{K} 538 \mathrm{R}}$ Mutant Cells}

Bmal1 ${ }^{\mathrm{K} 538}$ mutant cell lines were generated using CRISPR/Cas9-based genome editing. Fibroblasts stably expressing a clock-driven luciferase reporter (Bmall-LUC) (Nagoshi et al., 2004) were co-transfected with an all-in-one plasmid pSpCas9(BB)-2A-Puro 
(PX459) harboring CAS9, puromycin-resistance gene, and guide RNA (sgRNA), and a single-stranded oligodeoxynucleotide (ssODN) template comprising the point mutation. The sequence of ssODN was 5'-GCTCCAGCCCATTGAACATCACGA GTACGCCTCCCCCTGATGCCTCTTCTCCAGGcGGacAGAAGGTAAGACTGATG ATTCTTAGCCTAAGCTAGAGAACCTCTTGCCCAAGATCTGAAA-3' (lowercase letters indicate the mutation sites). The sgRNA was designed using the online tool (http://crispr.mit.edu/). The target sequence of sgRNA was 5'GGTCCTCCGTTCTTCCATTGTA-3' (PAM sequence is underlined). Transfected cells were selected with $1 \mu \mathrm{g} / \mathrm{ml}$ puromycin (Alfa Aesar) for two days and clonal cell lines were isolated by limiting dilution. Genomic DNA of these clones was extracted and subjected to sequencing. Targeting strategy for the Bmall locus is provided in Figure 8.

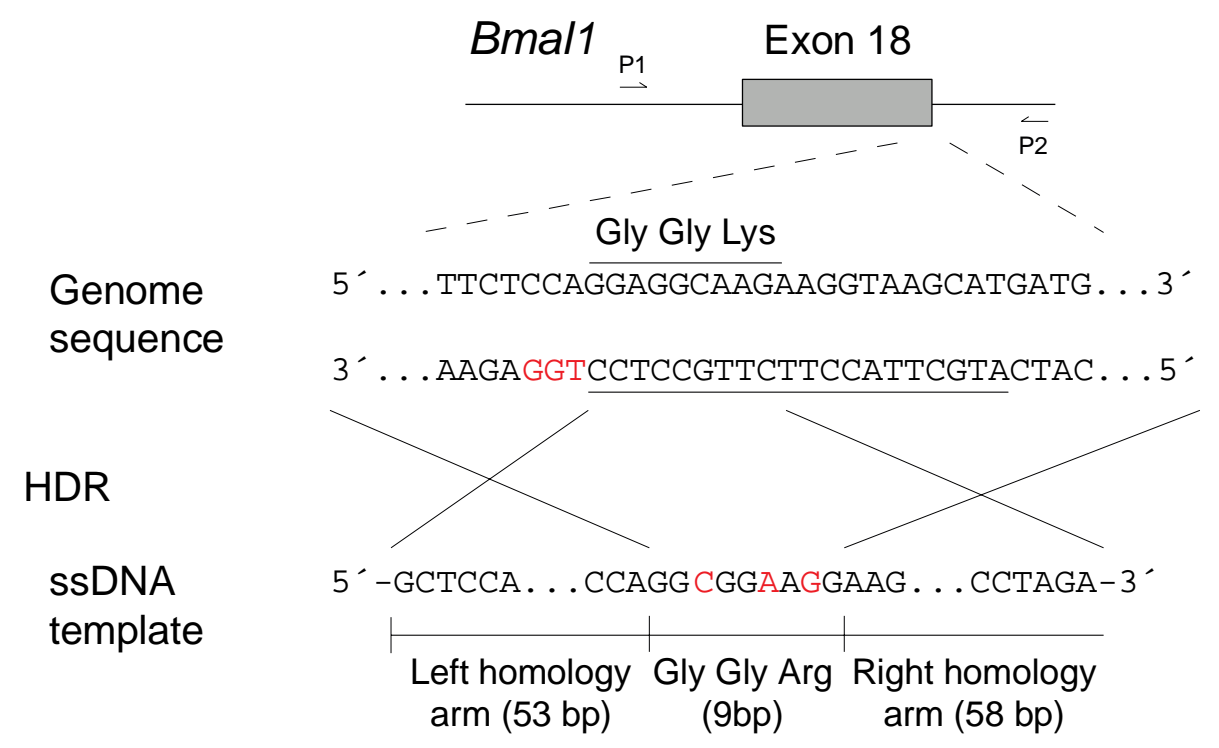

Figure 8: CRISPR/Cas9-mediated Generation of BMAL1 ${ }^{\text {K538R }}$ Mutant Cells.

Schematic representation of the gene targeting strategy for generating BMAL1 ${ }^{\mathrm{K} 538 \mathrm{R}}$ mutant cells using the CRISPR/Cas9 system. The gRNA-targeting sequence is underlined and the PAM sequence is indicated in red. The oligonucleotide donor (120 bp) is shown below the targeted site, with the Lys-to-Arg substitution indicated in red. Silent point mutations were introduced for the two adjacent Gly to prevent cutting of the donor DNA or re-cutting of the genome after homology-directed recombination (shown in red). The arrows indicate the position of primers used for sequencing (see Table 7). 


\subsubsection{Generation of loxP-STOP-loxP-Tip60-3xFLAG BAC and Experimental Animals}

The loxP-STOP-loxP-Tip60-3xFLAG BAC construct was generated by PolyGene Transgenics (Switzerland). A BAC clone (RP23-156A18, Children's Hospital Oakland Research Institute) containing the complete Tip60 locus was first modified by introducing a triple FLAG-tag at the C-terminus of Tip60 gene. Subsequently, a loxP-flanked STOP cassette was introduced downstream of the first exon. The construct was injected into pronuclei of fertilized eggs, and transgenic founders were screened for loxP-flanked STOP cassette and 3xFLAG integration. The final transgene construct is shown in Figure 9.
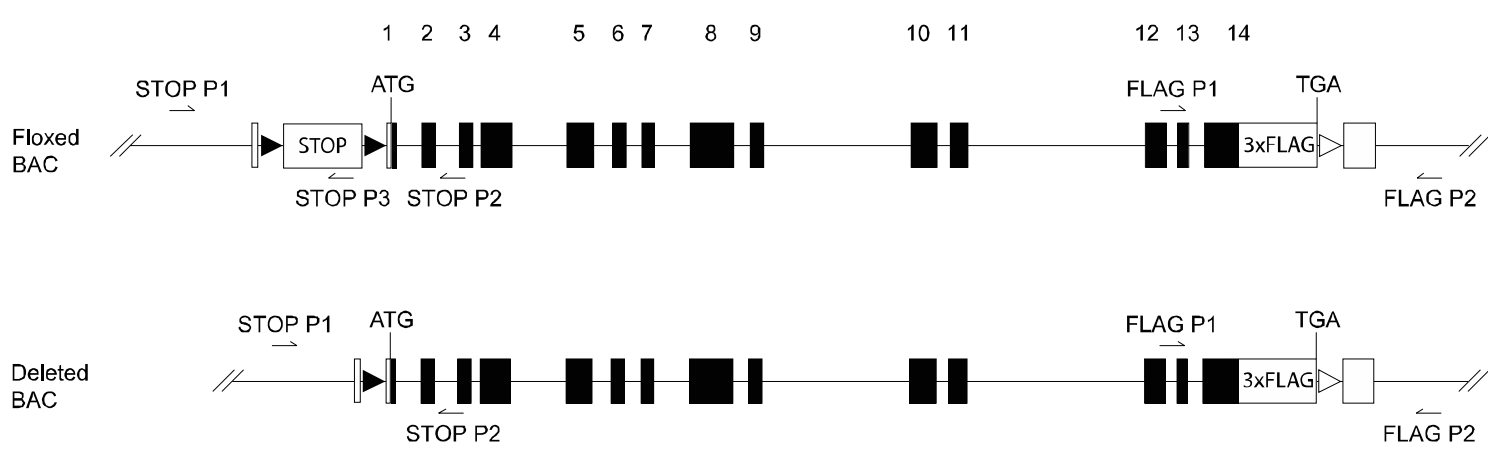

Figure 9: The loxP-STOP-loxP-Tip60-3xFLAG BAC Transgene.

The floxed transgene and the mutant transgene created by Cre-mediated recombination are shown. LoxP (black triangle) and FRT (open triangle) sites are marked. The arrows indicate the position of diagnostic primers STOP P1-P3 and FLAG P1-P2 (see Table 7).

To generate liver specific Tip60-3xFLAG mice for biochemical analysis, transgenic loxPSTOP-loxP-Tip60-3xFLAG (LSL-Tip60-FLAG)) mice were first crossed to Tip60 flffl mice. Transgene-positive Tip6 $60^{f l f l}$ mice were then used for breeding with a AlbCre driver mouse line (Postic et al., 1999) resulting in Cre-mediated deletion of TIP60 and the STOP cassette in the liver. Thus, endogenous TIP60 was replaced by a FLAG-tagged version of TIP60 exclusively in the liver.

\subsection{Immunohistochemistry}

Cryo-sectioning was carried out by technical staff and coronal sections of $10 \mu \mathrm{m}$ were produced. Cryo-sections through the SCN were fixed with $4 \%$ paraformaldehyde for 15 min at $4{ }^{\circ} \mathrm{C}$, boiled several times in $10 \mathrm{mM}$ sodium citrate $(\mathrm{pH} \mathrm{6.4)}$ for $5 \mathrm{~min}$, and permeabilized for 15 min with $0.5 \%$ Triton-X-100. Sections were blocked with $10 \%$ FBS in PBS followed by incubation with anti-TIP60 antibody (Frank et al., 2003) and a 
fluorescent secondary antibody (Thermo Fisher Scientific). Nuclei were stained with DAPI and slides were mounted with Vectashield mounting medium (Vector Laboratories).

\subsection{Molecular Biological Methods}

\subsubsection{Plasmids}

Cry2, Bmal1, Tip60 ORFs were amplified by PCR and cloned into pcDNA3.1 vector containing either C-terminal myc- or V5-tag. Cryl ORF was cloned in a $p C M V$-flag vector. Single site mutations were introduced by using the QuikChange II Site-directed Mutagenesis Kit (Agilent Technologies). Tip60 ${ }^{V 5}$ and Tip60 ${ }^{\text {C369A;E403Q;V5 were cloned }}$ into pLenti PGK Hygro DEST vector. Lentiviral Bmall-dLuc reporters were from S.A. Kay (USC Los Angeles). pBABE-puro SV40 LT (\#13970), pCL-Eco (\#12371), pMD2.G (\#12259), psPAX2 (\# 12260), and pLenti PGK Hygro DEST (\#19066) plasmids were from Addgene. Plasmids used in this study are listed in Table 3.

Table 3: Recombinant DNA

\begin{tabular}{|l|l|}
\hline Resource & Source \\
\hline pcDNA3.1/TIP60-V5 & This study \\
\hline pcDNA3.1/TIP60C369A;E403Q-V5 & This study \\
\hline pcDNA3.1/BMAL1-myc & This study \\
\hline pcDNA3.1/BMAL1K538R-myc & This study \\
\hline pCMV/CRY1-flag & This study \\
\hline BMAL1-flag & This study \\
\hline myc-CLOCK & Gift by M.J. Rossner (LMU Munich) \\
\hline myc-CLOCKmutA & Gift by P. Sassone-Corsi (UC Irvine) \\
\hline pLenti PGK Hygro DEST (w530-1) & Gift by P. Sassone-Corsi (UC Irvine) \\
\hline pLenti PGK Tip60-V5 & Addgene Plasmid \#19066 \\
\hline pLenti PGK TIP60C369A;E403Q-V5 & This study \\
\hline pLV6-Bmal1-dLuc & This study \\
\hline pBABE-puro SV40 LT & (Liu et al., 2008) \\
\hline pCL-Eco & Addgene Plasmid \#13970 \\
\hline pMD2.G & Addgene Plasmid \#12371 \\
\hline psPAX2 & Addgene Plasmid \#12259 \\
\hline pSpCas9(BB)-2A-Puro (PX459) & Addgene Plasmid \#12260 \\
\hline & Addgene Plasmid \#48139 \\
\hline
\end{tabular}




\begin{tabular}{|l|l|}
\hline pL451 & NCI at Frederick \\
\hline pL452 & NCI at Frederick \\
\hline TIP60 BAC (bMQ-331N14) & Wellcome Trust Sanger Institute \\
\hline TIP60 targeting construct & This study \\
\hline TIP60 BAC (RP23-156A18) & Children's Hospital Oakland Research Institute \\
\hline LSL-Tip60-FLAG BAC & This study \\
\hline
\end{tabular}

\subsubsection{Cell Culture}

Tip60 ${ }^{f /-}$ Mouse embryonic fibroblasts (MEFs) were isolated following standard procedures (Durkin et al., 2013) from day 13.5 embryos. MEFs, fibroblasts (NIH-3T3 Bmal1-LUC (Nagoshi et al., 2004) and CryWT/CryDKO (Gauger and Sancar, 2005), and HEK293T cells were cultured and passaged in standard medium (DMEM, high glucose, GlutaMAX supplement, pyruvate; $10 \%$ fetal bovine serum; $100 \mathrm{U} / \mathrm{ml}$ penicillin and 100 $\mu \mathrm{g} / \mathrm{ml}$ streptomycin [all Thermo Fisher Scientific]) at $37{ }^{\circ} \mathrm{C}$ in a humidified incubator with $5 \% \mathrm{CO}_{2}$. MEFs and fibroblasts were synchronized by treatment with $100 \mathrm{nM}$ dexamethasone (Sigma-Aldrich), a synthetic glucocorticoid that induces expression of Perl gene (Reddy et al., 2009, 2012). 500 nM JQ1 (Cayman Chemical), 300 nM flavopiridol (Santa Cruz Biotechnology) or vehicle (final 0.05\% DMSO) were added to standard medium after synchronization.

\subsubsection{Transfection and Viral Transduction}

HEK293T cells were transiently transfected using $1 \mathrm{mg} / \mathrm{ml}$ polyethylenimine solution (Sigma-Aldrich). After two days, cells were used for Western blotting and immunoprecipitations (IPs). Tip60 $0^{f /-}$ MEFs were immortalized with SV40 LT retrovirus. Immortalized MEFs were grown to confluence and transduced with AdGFP or AdCre adenoviruses (Vector Development Laboratory, Baylor College of Medicine, Houston, TX, USA) in standard medium containing 3\% fetal bovine serum (low-serum) and 8 $\mu \mathrm{g} / \mathrm{ml}$ polybrene (Sigma-Aldrich). After two days, medium was changed to low-serum medium and cells were cultured for another 4 - 6 days. For lentiviral transduction, viruses were produced according to standard protocol (Ramezani and Hawley, 2002) and concentrated using Lenti-X Concentrator (Clontech). MEFs were infected in low-serum medium supplemented with $8 \mu \mathrm{g} / \mathrm{ml}$ polybrene (Sigma-Aldrich) and transduced cells were selected with $10 \mu \mathrm{g} / \mathrm{ml}$ hygromycin B (Alfa Aesar) or $10 \mu \mathrm{g} / \mathrm{ml}$ blasticidin (Alfa Aesar) and further propagated. 


\subsubsection{Knock-Down Experiment}

Tip60 ${ }^{-/}$; Tip60 ${ }^{V 5}$ MEFs were reverse transfected with siRNA. AllStars Negative Control siRNA (Qiagen) was used as scrambled control. For depletion of Cry1 or Cry2, a mix of FlexiTube GeneSolution siRNAs (Qiagen) was used. Briefly, 1 x 106 cells were seeded in $10-\mathrm{cm}$ dishes in standard medium without antibiotics. siRNA mix was added to the OptiMEM, Lipofectamine 2000 solution (Thermo Fisher Scientific) to a final concentration of $20 \mathrm{nM}$, incubated for $20 \mathrm{~min}$ at room temperature, and added to the cells. On the next day medium was changed to standard medium and cells were allowed to grow for an additional 3 days before harvesting for ChIP.

\subsubsection{Preparation of Cell Extracts and Nuclear Extracts}

Transiently transfected HEK293T cells were lysed in $50 \mathrm{mM}$ Tris-HCl, pH 8.0; $150 \mathrm{mM}$ $\mathrm{NaCl} ; 5$ mM EDTA; 15 mM MgCl $2 ; 1 \%$ NP40; 1 mM DTT; 10 mM NaF; 10 mM NAM; Complete EDTA-free Protease Inhibitor Cocktail (Roche) for $30 \mathrm{~min}$ on ice and lysate was cleared by centrifugation $\left(20,000 \mathrm{x} \mathrm{g}, 4{ }^{\circ} \mathrm{C}, 10 \mathrm{~min}\right)$. Samples were boiled in SDS sample buffer or used for IP. Anti-Myc or anti-V5 conjugated agarose beads (SigmaAldrich) were used to precipitate myc- and V5-tagged proteins, respectively. The precipitates were washed three times with lysis buffer and bound proteins eluted by denaturation in SDS sample buffer.

Cells were fractionated as described (Méndez and Stillman, 2000). Briefly, cells were suspended in Buffer A (10 mM HEPES, pH 7.9; $10 \mathrm{mM} \mathrm{KCl} ; 1.5 \mathrm{mM} \mathrm{MgCl} 2 ; 0.34 \mathrm{M}$ sucrose; $10 \%$ glycerol; 1 mM DTT; Complete EDTA-free protease inhibitor cocktail [Roche]). Triton X-100 was added to a final concentration of $0.1 \%$ and cells were incubated for $8 \mathrm{~min}$ on ice. Nuclei were collected by centrifugation $\left(1,300 \mathrm{x} \mathrm{g}, 4{ }^{\circ} \mathrm{C}, 5\right.$ $\mathrm{min}$ ) and supernatant (fraction S1) was cleared by centrifugation $\left(20,000 \mathrm{x} \mathrm{g}, 4^{\circ} \mathrm{C}, 5 \mathrm{~min}\right.$ ) and designated as S2. Nuclei were washed with Buffer A and lysed in Buffer B (3 mM EDTA; 0.2 mM EGTA; 1 mM DTT; Complete EDTA-free Protease Inhibitor Cocktail [Roche]) for $30 \mathrm{~min}$ on ice. Chromatin was pelleted by centrifugation $\left(1,700 \mathrm{x} \mathrm{g}, 4^{\circ} \mathrm{C}, 5\right.$ min) and supernatant collected and designated as S3. Chromatin (P3) was washed once with Buffer B and denatured in SDS sample buffer. Samples were analyzed by Western blotting.

Nuclear extracts from HEK293T, fibroblasts and MEFs were prepared as described (Dimauro et al., 2012). Cells were washed twice with ice cold PBS and lysed with buffer STM (50 mM Tris-HCl, pH 7.4; 5 mM MgCl $2 ; 250$ mM sucrose; 10 mM NAM; Complete 
EDTA-free Protease Inhibitor Cocktail [Roche]) for $30 \mathrm{~min}$. Nuclei were collected by centrifugation ( $800 \mathrm{x} \mathrm{g}, 4{ }^{\circ} \mathrm{C}, 15 \mathrm{~min}$ ) and washed once with STM buffer. Nuclei were collected by centrifugation ( $500 \mathrm{x} \mathrm{g}, 4{ }^{\circ} \mathrm{C}, 15 \mathrm{~min}$ ) and resuspended in buffer NET (20 mM HEPES pH 7.9; 1.5 mM MgCl $2 ; 500$ mM NaCl; 0.2 mM EDTA; 20\% glycerol; $1 \%$ Triton-X-100; 10 mM NAM; Complete EDTA-free Protease Inhibitor Cocktail [Roche]). Samples were vortexed at maximum speed for $15 \mathrm{~s}$ and incubated on ice for $30 \mathrm{~min}$. Nuclei were lysed with 15 passages through an 18-gauge needle, sonicated using Bioruptor (30 sec ON / $30 \mathrm{sec} \mathrm{OFF})$ and cleared by centrifugation $\left(9,000 \mathrm{~g}, 4{ }^{\circ} \mathrm{C}, 30 \mathrm{~min}\right)$. For Western blotting samples were denatured in SDS sample buffer. For IPs samples were diluted 3-fold in dilution buffer (20 mM HEPES pH 7.9; $1.5 \mathrm{mM} \mathrm{MgCl}_{2} ; 0.2 \mathrm{mM}$ EDTA; 20\% glycerol; 10 mM NAM; Complete EDTA-free Protease Inhibitor Cocktail [Roche]) and incubated for 2 hours at $4{ }^{\circ} \mathrm{C}$ on a rotating wheel. Antibody was added and samples were put back on the rotating wheel. The next day, antibody-protein complexes were collected by Dynabead Protein G Beads (Thermo Fisher Scientific) and washed three times with wash buffer (20 mM HEPES pH 7.9; $1.5 \mathrm{mM} \mathrm{MgCl} 2 ; 166 \mathrm{mM} \mathrm{NaCl} ; 0.2 \mathrm{mM}$ EDTA; 20\% glycerol; 0,33\% Triton-X-100; 10 mM NAM; Complete EDTA-free Protease Inhibitor Cocktail [Roche]). Proteins were eluted by boiling in SDS sample buffer and analyzed by Western blotting.

Nuclear extracts from liver samples were prepared using NE-PER Nuclear and Cytoplasmic Extraction Reagents (Thermo Fisher Scientific) according to manufacturer's instructions. Briefly, samples were homogenized using a Dounce homogenizer in ice cold CRE I buffer and incubated on ice for $15 \mathrm{~min}$. Nuclei were collected by centrifugation $\left(16,000 \times \mathrm{g}, 4{ }^{\circ} \mathrm{C}, 5 \mathrm{~min}\right)$ and resuspended in NER buffer. Samples were vortexed at maximum speed for $15 \mathrm{~s}$ every $10 \mathrm{~min}$, for a total of $60 \mathrm{~min}$ and cleared by centrifugation $\left(16,000 \mathrm{~g}, 4{ }^{\circ} \mathrm{C}, 10 \mathrm{~min}\right)$. For Western blotting, samples were denatured in SDS sample buffer. For IPs antibody was added and samples were put on the rotating wheel. The next day, antibody-protein complexes were collected by Dynabead Protein G Beads (Thermo Fisher Scientific) and washed three times with wash buffer (10 mM Tris-HCl, pH 8.0; $150 \mathrm{mM} \mathrm{NaCl} ; 5 \mathrm{mM}$ EDTA; $15 \mathrm{mM} \mathrm{MgCl} 2 ; 1 \%$ NP40; $1 \mathrm{mM}$ DTT; $10 \mathrm{mM} \mathrm{NaF} ; 10$ mM NAM; Complete EDTA-free Protease Inhibitor Cocktail [Roche]). Proteins were eluted by boiling in SDS sample buffer and analyzed by Western blotting. 


\subsubsection{Western Blotting}

Samples were resolved on 4-15\% gradient TGX precast gels (Bio Rad) and proteins were transferred onto PVDF membranes using the Trans-Blot Turbo Transfer System (Bio Rad). Membranes were blocked with $5 \%$ non-fat milk or 3\% BSA and incubated with primary antibodies overnight at $4{ }^{\circ} \mathrm{C}$. Membranes were incubated for 2 hours at room temperature with appropriate HRP-conjugated secondary antibodies and developed with SuperSignal Chemiluminescent Substrate (Thermo Fisher Scientific). Signal intensities were determined on an ImageQuant LAS4010 imager (GE Healthcare) and quantified using ImageQuant software (GE Healthcare). Antibodies against CDK9 (sc-484) and Actin (sc-1616-HRP) were purchased from Santa Cruz Biotechnology, BRD4 (A301985A) from Bethyl, BMAL1 (NB100-2288) from Novus, acetylated BMAL1 (AB15396) from Millipore, TBP (ab197874-HRP) from Abcam, FLAG M2 (A8592-HRP) from Sigma-Aldrich, V5 (R961-25-HRP) and myc (R951-25-HRP) from Thermo Fisher Scientific, GST (RPN1236-HRP) from GE Healthcare, acetylaed Lysine (9441S), goat anti-rabbit HRP (7074) and mouse anti-rabbit (conformation specific) HRP (5127S) from Cell Signaling Technology, and goat anti-rat HRP (ab97180) and goat anti-guinea pig HRP (A18775) from Abcam. Antibodies against TIP60 were provided by Dr. J.W. Lough (Fisher et al., 2016) and Dr. B. Amati (Frank et al., 2003), against CRY1 by Dr. J.A. Ripperger (Stratmann et al., 2010), and against CRY2 by Dr. J.S. Takahashi (Koike et al., 2012).

\subsubsection{Chromatin Immunoprecipitation (ChIP)}

For BMAL1 (NB100-2288; Novus), acetylated BMAL1 (AB15396; Millipore), BRD4 (A301-985A; Bethyl), CDK9 (sc-484 and sc-13130; Santa Cruz Biotechnology), Pol II (MABI0601; BIOZOL), Ser2 phosphorylated Pol II (04-1571; Millipore) and TFIIE $\alpha$ (ab28177; Abcam) ChIP analysis were performed as described (Lin et al., 2012; Rahl et al., 2010) with minor modifications. Fibroblasts were crosslinked with $1 \%$ formaldehyde for $10 \mathrm{~min}$ at room temperature. The reaction was stopped by adding $125 \mathrm{mM}$ glycine and incubating for $5 \mathrm{~min}$. Cells were washed twice with PBS, scraped and frozen in liquid nitrogen. Dynabead Protein G Beads (Thermo Fisher Scientific) were blocked with $0.5 \%$ BSA (w/v) in PBS and bound to the indicated antibodies. Crosslinked cells were lysed in lysis buffer 1 (50 mM HEPES pH 7.3; 140 mM NaCl; 1 mM EDTA; 10\% glycerol; 0.5\% NP-40; $0.25 \%$ Titon-X-100; 10 mM NAM; Complete EDTA-free Protease Inhibitor Cocktail [Roche]; PhosSTOP Phosphatase Inhibitor Cocktail [Roche]) and washed with 
lysis buffer 2 (10 mM Tris- $\mathrm{HCl} \mathrm{pH} 8.0 ; 200$ mM NaCl; 1 mM EDTA; 0.5 mM EGTA; 10 mM NAM; Complete EDTA-free Protease Inhibitor Cocktail [Roche]; PhosSTOP Phosphatase Inhibitor Cocktail [Roche]). Pellets were resuspended and sonicated in lysis buffer 3 (50 mM Tris-HCl pH 7.5; 140 mM NaCl; 1 mM EDTA; 1 mM EGTA; $1 \%$ TritonX-100; 0.1\% SDS; 0.1\% Na-deoxycholate; 10 mM NAM; Complete EDTA-free Protease Inhibitor Cocktail [Roche]; PhosSTOP Phosphatase Inhibitor Cocktail [Roche]) (Bioruptor; $30 \mathrm{sec}$ ON / $30 \mathrm{sec}$ OFF) to obtain chromatin fragments of 150-600 bp length. Sonicated lysates were cleared and incubated overnight at $4{ }^{\circ} \mathrm{C}$ with beads bound with antibody for indicated factors. Beads were washed twice (CDK9 and Pol II Ser2P) or three times (BMAL1, AcBMAL1, Pol II, and BRD4) with lysis buffer 3, once with lysis buffer 3 with $500 \mathrm{mM} \mathrm{NaCl}$, one time with $\mathrm{LiCl}$ wash buffer $(10 \mathrm{mM}$ Tris- $\mathrm{HCl} \mathrm{pH}$ 8.0; $250 \mathrm{mM} \mathrm{LiCl} ; 1 \mathrm{mM}$ EDTA; 0.5\% NP-40; 0.5\% (50 mM Tris-HCl pH 7.5; $140 \mathrm{mM}$ $\mathrm{NaCl} ; 1$ mM EDTA; 1 mM EGTA; $1 \%$ Triton-X-100; 0.1\% SDS; 0.1\% Na-deoxycholate) and one time with TE plus $50 \mathrm{mM} \mathrm{NaCl}$. DNA was eluted in elution buffer (50 mM Tris$\mathrm{HCl} \mathrm{pH} 8.0 ; 10 \mathrm{mM}$ EDTA; 1\% SDS). The eluted complexes were de-crosslinked overnight and RNA and protein were digested using RNase A (Qiagen) and Proteinase K (Thermo Fisher Scientific). Immunoprecipitated DNA fragments were purified by phenol chloroform extraction and ethanol precipitation and used for qPCR analysis.

For TIP60 ${ }^{\mathrm{V} 5}$ (R960-25; Thermo Fisher Scientific), CRY1 (Ye et al., 2011) and CRY2 (Koike et al., 2012) in cells and BMAL1 (NB100-2288; Novus) and acetylated BMAL1 (AB15396; Millipore) in liver ChIP assays were performed as described (Adli and Bernstein, 2011; Nelson et al., 2006) with minor modifications. Cells and liver were cross-linked with $1 \%$ formaldehyde for $15 \mathrm{~min}$ at room temperature. For dual crosslinking (Zeng et al., 2006) Tip60 ${ }^{-/}$; Tip60 V5 MEFs were fixed with $1.5 \mathrm{mM}$ ethylene glycol bis(succinimidylsuccinate) (EGS) (Thermo Fisher Scientific) for $30 \mathrm{~min}$ followed by $1 \%$ formaldehyde for another $15 \mathrm{~min}$. After quenching the reaction by adding glycine to a final concentration of $125 \mathrm{mM}$, cells were lysed in SDS lysis buffer (50 mM Tris$\mathrm{HCl}$, pH 8.1; $100 \mathrm{mM} \mathrm{NaCl} ; 5$ mM EDTA; 1\% SDS; Complete EDTA-free Protease Inhibitor Cocktail [Roche]) on ice for $10 \mathrm{~min}$, with brief vortexing. Liver was homogenized using a Dounce homogenizer in SDS lysis buffer and incubated on ice for 15 min. Samples were diluted 10-fold in ChIP dilution buffer $(16.7 \mathrm{mM}$ Tris-HCl, pH 8.1; 167 mM NaCl, 1.2 mM EDTA; 1.1\% Triton X-100; 0.01\% SDS; Complete EDTAfree Protease Inhibitor Cocktail [Roche]) and sonicated (Bioruptor; $30 \mathrm{sec}$ ON / $30 \mathrm{sec}$ OFF) to obtain chromatin fragments of 150 - 600 bp length. Appropriate antibodies were 
added to the chromatin solution and incubated overnight at $4{ }^{\circ} \mathrm{C}$ on a rotating wheel. For anti-CRY2 antibody Dynabead Protein A Beads (Thermo Fisher Scientific) were used, for anti-V5, BMAL1, and acetylated BMAL1 antibodies Dynabead Protein G Beads (Thermo Fisher Scientific) were used, and for anti-CRY1 antibody Protein L Magnetic Beads (Pierce) were used. Immunoprecipitated chromatin complexes were washed twice with ice cold low-salt wash buffer $(20 \mathrm{mM}$ Tris- $\mathrm{HCl}, \mathrm{pH} 8.1 ; 150 \mathrm{mM} \mathrm{NaCl} ; 2 \mathrm{mM}$ EDTA; $1 \%$ Triton X-100; 0.1\% SDS), twice with ice cold lithium chloride wash buffer (10 mM Tris-HCl, pH 8.1; 250 mM LiCl; 1 mM EDTA; 1\% NP40; 1\% deoxycholate), and twice with ice cold TE buffer (10 mM Tris-HCl, pH 8; 1 mM EDTA). $100 \mu 1$ 10\% Chelex 100 Slurry (Bio Rad) was added directly to the washed beads, briefly vortexed, and boiled for $10 \mathrm{~min}$. Input DNA samples were ethanol precipitated and washed with $70 \%$ ethanol. DNA pellets were dissolved in $100 \mu 1$ 10\% Chelex 100 suspension and boiled for $10 \mathrm{~min}$. Samples were proteinase K (Thermo Fisher Scientific) treated and boiled for $10 \mathrm{~min}$. Supernatant was transferred to a new tube and used for qPCR analysis. For validation of the antibodies, the $D b p$ gene was used and deficient cells or antibody isotype controls were applied to evaluate the ChIP-grade quality of the antisera (Figure 10). Primer pair that targets an unbound region was used as internal control (Duong et al., 2011) and fold enrichment was calculated using the $2^{\wedge}(-\Delta \Delta C T)$ method. Experiments were performed with biological independent triplicates and data normalized to the mean value obtained from the locus with the strongest enrichment. Primer sequences for ChIPqPCR are listed in Table 4. 

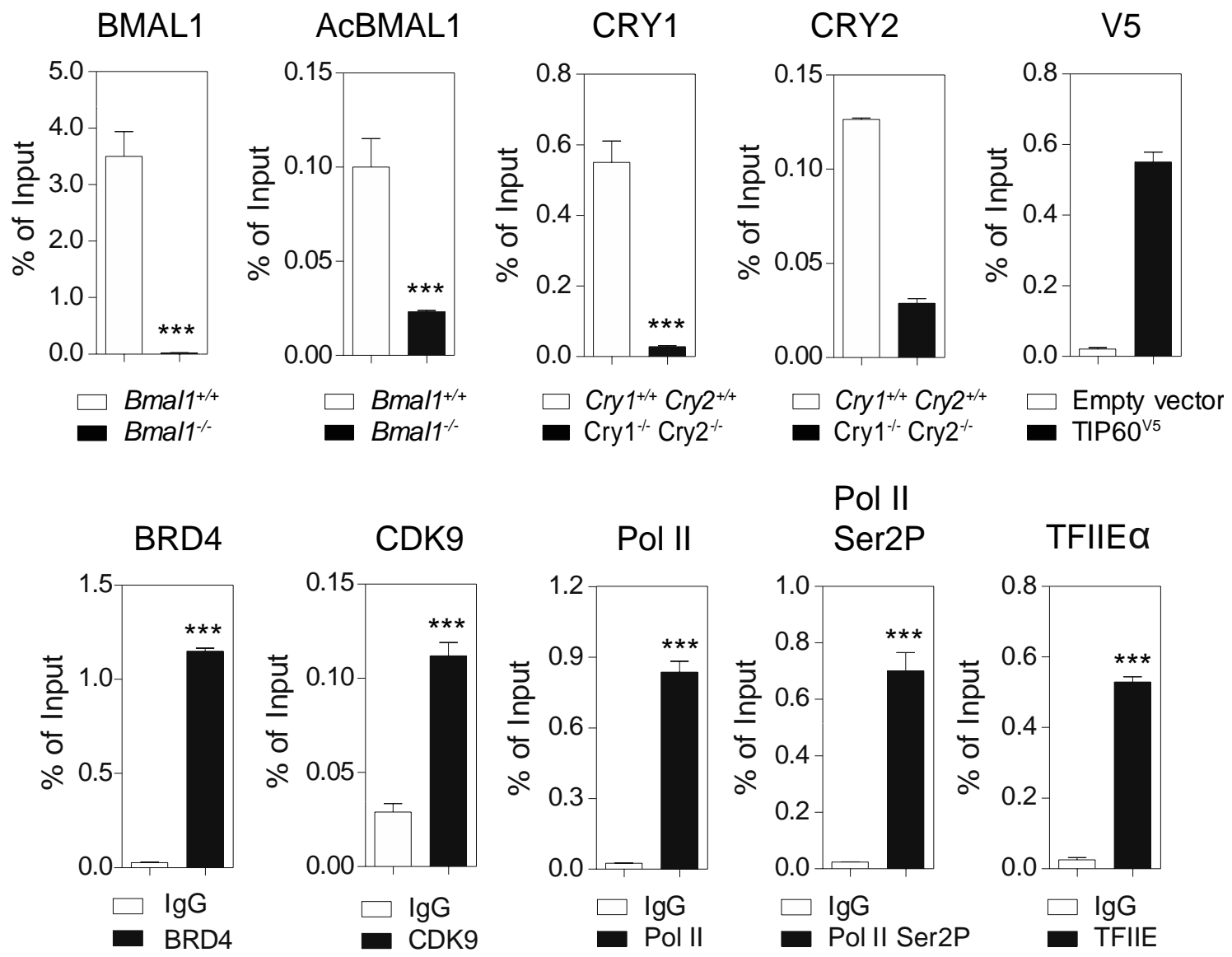

Pol II

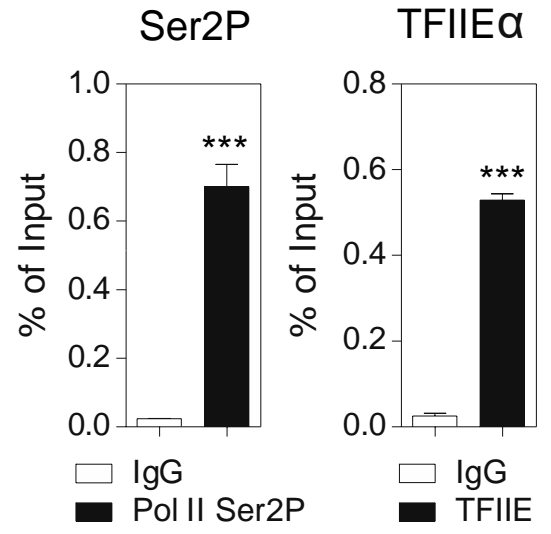

Figure 10: Validation of Antibodies used for ChIP Studies.

Deficient cells when possible or antibody isotype controls were used to validate the ChIPgrade quality of the antisera. The test gene used was $\mathrm{Dbp}$. Data are shown as mean $\pm \mathrm{SEM}$ ( $\mathrm{n}=3 ; * * \mathrm{p}<0.01$, and $* * * \mathrm{p}<0.001$ Student's t-test).

Table 5: Primer Sequences used for ChIP-qPCR analysis

\begin{tabular}{|c|c|c|}
\hline Target & Forward (5' - 3') & Reverse (5' - 3') \\
\hline$D b p-0.4$ & ACACCCGCATCCGATAG & CCACTTCGGGCCAATGAG \\
\hline Dbp - -0.2 & ACACAAGTTCAGCCCCTCAC & GGCAAGAACCAATCACGTCT \\
\hline Dbp TSS & AAGCTCCTTTCTTTGCGAGA & TCAAGCAGCTGTCTCTTTGC \\
\hline$D b p+0.2$ & TCTGCAGAGCAGACTGGTTGA & GCGTGCAAACCTCCAGGAT \\
\hline$D b p+0.8$ & ATGCTCACACGGTGCAGACA & CTGCTCAGGCACATTCCTCAT \\
\hline$D b p+2.4$ & TGGGACGCCTGGGTACAC & GGGAATGTGCAGCACTGGTT \\
\hline$D b p+4.4$ & AAGAACAATGAAGCAGCCAAGAG & GGCAGCCCGCACAGATAT \\
\hline$D b p+5.2$ & GGCACCGGAGTAGGCAAGA & GCCTGGAATGTATGAGCTAGCA \\
\hline Perl - 1.5 & CACCATGCCCAGCTTAGAA & CACCTTTGGAAGAGCAGTCA \\
\hline Perl -0.5 & GGCACCAGAAACCTCTTGTA & CCTTGACGACACTTACCCAATA \\
\hline Per1 TSS & GTGTGAGGGATCATGCAAATG & CTAGAGTTGAACTTGTGGTCTCT \\
\hline Perl +0.5 & GGATTCTGAAAGGGAGGATAAGG & CAGAGCAGTCCTTTGGTAACT \\
\hline
\end{tabular}




\begin{tabular}{|c|c|c|}
\hline Perl +4.9 & CCGAGGCCTCAGAGTCCCA & GGCCATCTCTTACACCCCTT \\
\hline Perl +14.3 & TGGACAGACCACTTCAGCAG & GAAGTTCTCAGCCCCATTTG \\
\hline $\operatorname{Rev}-E r b \alpha-2.1$ & AGCTAGTGAGAAGAGGGAACTA & GCTTCATACCTCACACAGACTAC \\
\hline Rev-Erb $\alpha-0.5$ & ACAAGCTGGGAGGAGAGAT & GGGTTAGGGTGAAGAAGAGAAAG \\
\hline Rev-Erb $\alpha$ TSS & AGGCACACTCCACCTACATTGTCA & TGGAGCAGGTACCATGTGATTCCA \\
\hline $\operatorname{Rev}-\operatorname{Erb} \alpha+0.1$ & CGCTCGGAACTGTGAGTAG & GGCATTTGTTCTGCAGCTAAT \\
\hline$R e v-E r b \alpha+2.0$ & TCATGCCCTCTTTCAGGATT & TTACCCGGCTATGGTTTCAC \\
\hline Rev-Erb $\alpha+6.8$ & СTCCTTCTCCTTTCCCGTTG & CCTGAGCTCCTCCTCTGACA \\
\hline Control & TGCTAGATGCTGCGGAAGAACTGA & TCTCTATGCTCCCAGCCAAGGTAT \\
\hline
\end{tabular}

\subsubsection{RNA Analysis by Quantitative Real-Time PCR (qPCR)}

Total RNA was extracted using RNeasy Plus Mini Kit (Qiagen). Cells were directly lysed in the vessel and liver samples were homogenized using the Bead Ruptor (Biolabs). cDNA was synthesized using Maxima H Minus First Strand cDNA Synthesis Kit (Thermo Fisher Scientific). Real-time PCR reactions were performed on a CFX96 RealTime PCR Detection System (Bio-Rad) with IQ SYBR Green Supermix (Bio-Rad). Eefla or Gapdh expression was used for normalization. Primers used for qPCR analysis are listed in Table 6.

Table 6: Primer Sequences used for qPCR analysis

\begin{tabular}{|l|l|l|}
\hline Target & Forward $\left(\mathbf{5},-\mathbf{3}^{\prime}\right)$ & Reverse $(\mathbf{5}, \mathbf{- 3})$ \\
\hline Eefla & TGCCCCAGGACACAGAGACTTCA & AATTCACCAACACCAGCAGCAA \\
\hline Gapdh & CATGGCCTTCCGTGTTCCTA & CCTGCTTCACCACCTTCTTGA \\
\hline $18 S$ & AGTCCCTGCCCTTTGTACACA & GATCCGAGGGCCTCACTAAAC \\
\hline ActB & TTGTCCCCCCAACTTGATGT & CCTGGCTGCCTCAACACCT \\
\hline $36 B 4$ & AGATGCAGCAGATCCGCAT & GTTCTTGCCCATCAGCACC \\
\hline D $p p$ & AATGACCTTTGAACCTGATCCCGCT & GCTCCAGTACTTCTCATCCTTCTGT \\
\hline Per 1 & AGTTCCTGACCAAGCCTCGTTAG & CCTGCCCTCTGCTTGTCATC \\
\hline Per 2 & GCCAAGTTTGTGGAGTTCCTG & CTTGCACCTTGACCAGGTAGG \\
\hline Rev-Erb $\alpha$ & AGCTCAACTCCCTGGCACTTAC & CTTCCTCGGAATGCATGTTGTTC \\
\hline Bmall & CCTAATTCTCAGGGCAGCAGAT & TCCAGTCTTGGCATCAATGAGT \\
\hline Tip 60 & GCCTGGACGGAAGCGGAAAT & CAATGTCGTGGCTCCGGT \\
\hline c-Fos & TTCCTGGCAATAGCGTGTTC & TTCAGACCACCTCGACAATG \\
\hline$c$-Jun & GAAGTGACGGACCGTTCTATGAC & GGAGGAACGAGGCGTTGAG \\
\hline
\end{tabular}




\subsubsection{Radioactive In Situ Hybridization}

In situ hybridization with ${ }^{35} \mathrm{~S}$-labeled antisense RNA probes was carried out on frozen sections through the SCN by Dr. Henrik Oster. Relative quantification of expression strength was performed by densitometric analysis of autoradiograph films. Templates for Dbp, Perl, and Bmall were as described (Oster et al., 2003, 2006).

\subsubsection{Genotyping}

Ear biopsies or cells were lysed in $10 \mathrm{mM}$ Tris- $\mathrm{HCl} \mathrm{pH} 8.3 ; 50 \mathrm{mM} \mathrm{KCl} ; 2.5 \mathrm{mM} \mathrm{MgCl}$; $0.1 \mathrm{mg} / \mathrm{ml}$ gelatin; $0.45 \% \mathrm{NP} 40 ; 0.45 \%$ Tween-20; $0.015 \mu \mathrm{g} / \mu 1$ Proteinase K (Thermo Fisher Scientific) over night or for $2 \mathrm{~h}$ at $55^{\circ} \mathrm{C}$ followed by Proteinase $\mathrm{K}$ inactivation at $85^{\circ} \mathrm{C}$ for $1 \mathrm{~h}$.

Genotyping PCR was performed using GoTaq Flexi DNA Polymerase (Promega) according to manufacturer's instructions and resolved by agarose gel electrophoresis. Both steps were mainly done by the technical staff. Primer sequences and PCR product sizes are listed in Table 7.

\section{Table 7: Primer Sequences used for Genotyping}

\begin{tabular}{|c|c|c|c|}
\hline PCR & Primer & Sequence $\left(5^{\prime}-3^{\prime}\right)$ & PCR product \\
\hline \multirow[t]{3}{*}{ Tip60 } & Tip60 Pl & TCAGAAGATGCACCTTCTGCTGG & WT: $331 \mathrm{bp}$ \\
\hline & Tip60 P2 & GGAAGGTTCAAAATTCCAGTAGGC & Floxed: 429 bp \\
\hline & Tip60 P3 & TGCTTCCGCTTCCTGAATGCTG & Deleted: 487 bp \\
\hline \multirow[t]{3}{*}{ Syt10 } & Syt10 fw & AGACCTGGCAGCAGCGTCCGTTGG & WT: 420 bp \\
\hline & Syt10 rev & AAGATAAGCTCCAGCCAGGAAGTC & KI: $550 \mathrm{bp}$ \\
\hline & $\begin{array}{l}\text { Syt10 KI } \\
\text { rev }\end{array}$ & & \\
\hline \multirow[t]{2}{*}{ Bmall } & Bmall P1 & TGGAGCACAGCTGCACTTAT & $438 \mathrm{bp}$ \\
\hline & Bmall P2 & AAGTCCCAGCTGCACACAAG & \\
\hline \multirow[t]{3}{*}{ Clock } & Clock P1 & AGCTGGGGTCTATGCTTCCT & WT:280 bp \\
\hline & Clock P2 & CGCTGAGAGCCAAGACAAT & Floxed: $380 \mathrm{bp}$ \\
\hline & Clock P3 & CAGCTTCATTTGAAATCTGCAT & Deleted: 450 bp \\
\hline \multirow[t]{2}{*}{$B A C-F L A G$} & FLAG P1 & AGTGAGATCAGTGAAATCACTAG & WT: 573 bp \\
\hline & $F L A G P 2$ & CTGTGTACATTAAGATGGGTGG & FLAG: 726 bp \\
\hline \multirow[t]{3}{*}{$B A C-S T O P$} & STOP P1 & CGTTCCTCCAATCGCTGTGCG & WT: 403 \\
\hline & STOP P2 & CGGCGCAACCAGGACAACGAA & Floxed: 649 bp \\
\hline & STOP P3 & CATCAATGTATCTTATCATGTCTG & Deleted: 479 bp \\
\hline Tip60-BAC & $B A C P 1$ & GTTGACAAGTCCGCTCCTTC & Tip60 WT: $652 \mathrm{~b}$ \\
\hline
\end{tabular}




\begin{tabular}{|c|c|c|c|}
\hline & $\mid \begin{array}{l}B A C P 2 \\
B A C P 3 \\
B A C P 4\end{array}$ & $\begin{array}{l}\text { GAACTGAGCGAACAAGTGCA } \\
\text { САTCTTCССТСССТCСАCTG } \\
\text { ССТGCАTGTCTGAACССАAC }\end{array}$ & $\begin{array}{l}\text { Tip60 floxed: } 750 \mathrm{bp} \\
\text { Tip60 deleted: } 434 \mathrm{bp} \\
\text { BAC floxed: } 1008 \mathrm{bp} \\
\text { BAC deleted: } 728 \mathrm{bp}\end{array}$ \\
\hline AlbCre & $\begin{array}{l}\text { Cre } P 1 \\
\text { Cre } P 2\end{array}$ & $\begin{array}{l}\text { CGATGCAACGAGTGATGAGGTTCG } \\
\text { AGCATTGCTGTCACTTGGTCGTGG }\end{array}$ & $\begin{array}{l}\text { pos: } 300 \mathrm{bp} \\
\text { neg: none }\end{array}$ \\
\hline
\end{tabular}

\subsubsection{TUNEL Assay}

TUNEL assay on sections through the SCN using an In Situ Cell Death Detection Kit (Roche) was performed according to manufacturer's instructions. As a positive control, sections were treated with DNaseI (New England Biolabs) for 10 min to induce DNA fragments and single strand breaks. Nuclei were stained with DAPI and slides were mounted with Vectashield mounting medium (Vector Laboratories).

\subsubsection{Bioluminescence Recording}

After synchronization of cells with dexamethasone medium was changed to recording medium (DMEM with high glucose, without glutamine and phenol red; $2 \mathrm{mM}$ GlutaMAX; 10 mM HEPES; 5\% FBS; $100 \mathrm{U} / \mathrm{ml}$ penicillin and $100 \mu \mathrm{g} / \mathrm{ml}$ streptomycin [all Thermo Fisher Scientific] and 0.1 mM D-luciferin [Synchem]). Petri dishes were sealed with transparent plastic film and bioluminescence was recorded in a LumiCycle luminometer (Actimetrics).

For JQ1 and flavopiridol dose dependence experiments, NIH-3T3 Bmall-LUC fibroblasts were plated on 35-mm dishes and cultured for 2 days to reach confluency. Cells were synchronized and medium was replaced with the recording medium containing various concentrations of JQ1 (Cayman Chemical) and flavopiridol (Santa Cruz Biotechnology) or vehicle (final $0.08 \%$ DMSO). For calculation of period, luminescence intensity, and damping rate parameters the curve fitting program LumiCycle (Actimetrics) was used and data obtained from the first day were excluded from analysis because of transient luminescence changes upon the medium change.

\subsubsection{In Vitro Acetylation Assay}

Recombinant TIP60 (SignalChem) and BMAL1 (Abnova) proteins were mixed in acetyltransferase assay buffer (50 mM Tris-HCl, $\mathrm{pH}$ 8.0; 0.1 mM EDTA; 0.4 mM DTT; $25 \mathrm{ng} / \mu \mathrm{l} \mathrm{BSA} ; 5 \%$ glycerol) in the presence or absence of $10 \mu \mathrm{M}$ acetyl-CoA (Sigma- 
Aldrich) and incubated for $1 \mathrm{~h}$ at $30^{\circ} \mathrm{C}$. The reaction was stopped by denaturation in SDS sample buffer and proteins were analyzed by Western blotting.

\subsubsection{Flow Cytometry}

Cells were stained using the SYTOX AADvanced Dead Cell Stain Kit (Thermo Fisher Scientific) according to the manufacturer's instructions and samples were analyzed using the Accuri C6 Flow Cytometer (BD). To provide a positive control cells were boiled for 3 min.

\subsection{Statistical Analysis}

Statistical analyses were performed using GraphPad Prism 5 software (GraphPad Software) and all data are presented as mean +/- SEM. For comparison between two groups a two-tailed student's t-test was applied. Significant differences between multiple groups were evaluated using two-way ANOVA with Bonferroni post-tests and results are listed in Table 8.

Table 9: Two-way ANOVA Statistical Analysis

\begin{tabular}{|c|c|c|c|c|c|}
\hline & \multirow[b]{2}{*}{ Gene } & \multicolumn{3}{|c|}{ two-way ANOVA main effects } & \multirow{2}{*}{\begin{tabular}{|l|} 
Bonferroni post-test \\
p-values below 0.05 are shown
\end{tabular}} \\
\hline & & Time (p) & Genotype (p) & Interaction (p) & \\
\hline \multirow[t]{3}{*}{ Figure 16} & $D b p$ & $<0.0001$ & $<0.0001$ & $<0.0001$ & Clone $1 / 2 / 3: 24 / 28 / 32 / 36 / 40 / 44:<0.001$ \\
\hline & Perl & $<0.0001$ & $<0.0001$ & $<0.0001$ & $\begin{array}{c}\text { Clone } 1: 24 / 28 / 32 / 36 / 44:<0.001 ; 40<0.01 \\
\text { Clone } 2: 24 / 28 / 44:<0.001 ; 32<0.05 \\
\text { Clone 3: } 24 / 28 / 40 / 44:<0.001 ; 32 / 36:< \\
0.05\end{array}$ \\
\hline & $\operatorname{Rev}-E r b \alpha$ & $<0.0001$ & $<0.0001$ & $<0.0001$ & $\begin{array}{c}\text { Clone } 1: 24 / 28 / 40 / 44:<0.001 ; 32<0.01 \\
\text { Clone } 2: 24 / 28 / 32 / 36 / 40 / 44:<0.001 \\
\text { Clone } 3: 24 / 28 / 32 / 36:<0.001\end{array}$ \\
\hline \multirow[t]{3}{*}{ Figure 20} & Perl & $<0.0001$ & $<0.0001$ & $<0.0001$ & CT 0/24: <0.01; CT 6: <0.0001 \\
\hline & Bmall & 0.0002 & 0.0005 & 0.2175 & CT 18: $<0.01$ \\
\hline & $D b p$ & $<0.0001$ & 0.0002 & 0.0006 & CT 6: $<0.0001 ;$ CT12: $<0.05$ \\
\hline Figure 21D & $D b p$ & $<0.0001$ & $<0.0001$ & $<0.0001$ & $24 / 28 / 32 / 36 / 40 / 44:<0.0001$ \\
\hline \multirow[t]{7}{*}{ Figure $21 \mathrm{~F}$} & $D b p$ & $<0.0001$ & $<0.0001$ & $<0.0001$ & 24/36/40: $<0.001 ; 28 / 32 / 44:<0.0001$ \\
\hline & Nrldl & $<0.0001$ & 0.0012 & $<0.0001$ & $24:<0.001 ; 28:<0.0001$ \\
\hline & Perl & 0.0002 & $<0.0001$ & 0.0002 & 24/28/32/36/40/44: $<0.0001$ \\
\hline & Per2 & $<0.0001$ & $<0.0001$ & $<0.0001$ & $24 / 28 / 32 / 36 / 44:<0.0001 ; 40:<0.01$ \\
\hline & Bmall & $<0.0001$ & $<0.0001$ & 0.0007 & $36 / 40:<0.0001 ; 44:<0.01$ \\
\hline & Gapdh & 0.2918 & 0.0079 & 0.5568 & - \\
\hline & Gene & Time (p) & Treatment (p) & Interaction (p) & p-values below 0.05 are shown \\
\hline Figure 12 & $D b p$ & $<0.0001$ & $<0.0001$ & $<0.0001$ & $24 / 28 / 32 / 36 / 40 / 44:<0.001$ \\
\hline
\end{tabular}




\section{Chapter 3: Results}

\subsection{Inhibition of CDK9 and BRD4 Abolishes Circadian Oscillations}

Synchronized fibroblasts stably expressing the clock-driven luciferase reporter BmallLUC (Nagoshi et al., 2004) show sustained reporter expression with a period of $\sim 26 \mathrm{~h}$. In the presence of flavopiridol (FP), a potent inhibitor of CDK9 subunit of P-TEFb (Chao and Price, 2001; Chao et al., 2000; Rahl et al., 2010), the amplitude of luciferase activity rhythms was suppressed in a dose dependent manner concomitant with an increased period length until, at high doses, the rhythm was fully lost (Figure 11 A and B). Treatment of fibroblasts with FP also strongly reduced endogenous mRNA expression levels of $\mathrm{Dbp}$, Perl, and Rev-Erb $\alpha$ (Figure 11C) that are direct CLOCK-BMAL1 targets (Stratmann et al., 2012). These findings suggest that circadian clock gene regulation not only occurs at
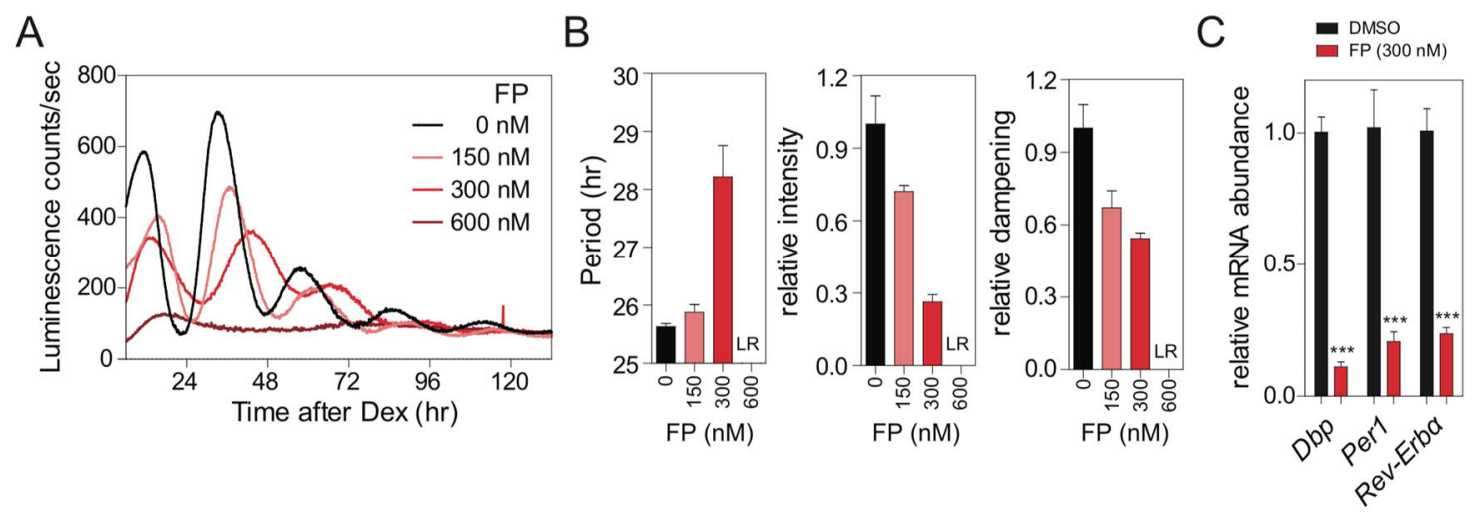

Figure 11: RNA Pol II Pause Release Is a Regulatory Step of Clock Gene Expression. (A) Bioluminescence recordings of dexamethasone (Dex) synchronized Bmall-LUC fibroblasts treated with increasing concentrations of flavoperidol (FP) $(n=4)$. (B) Change of period, luminescence intensity, and damping rate relative to DMSO control $(n=4)$. LR: loss of rhythm. (C) mRNA expression analysis of fibroblasts treated with either DMSO or flavopiridol (300 nM) carried out 24 hours after Dex synchronization. Data are shown as mean \pm SEM $(\mathrm{n}=3 ; * * * \mathrm{p}<0.001$, Student's t-test $)$.

the initiation of transcription (Koike et al., 2012; Le Martelot et al., 2012) but also at the level of RNA Pol II pause release and productive elongation. Triazolothienodiazepine (JQ1) is a potent and selective inhibitor of BRD4 (Shi and Vakoc, 2014). JQ1 treatment of Bmal1-LUC reporter fibroblasts dampened luciferase activity rhythms in a dosedependent manner and evoked a lengthening of the period (Figure 12A and B). This effect was reversible upon washout of JQ1 (Figure 12C). Thus, perturbation of circadian rhythms is due to dysfunction within the circadian system and not to cell toxicity or apoptosis. JQ1 also resulted in a strong dampening of the rhythm of endogenous $D b p$ 
expression in these cells (Figure 12D). In the presence of JQ1, enrichment of BRD4 and CDK9 at the TSS of Dbp was markedly reduced (Figure 12E). Ser2 phosphorylation of Pol II, at the 3'-end of $D b p$, was strongly diminished (Figure 12F) suggesting that RNA

A

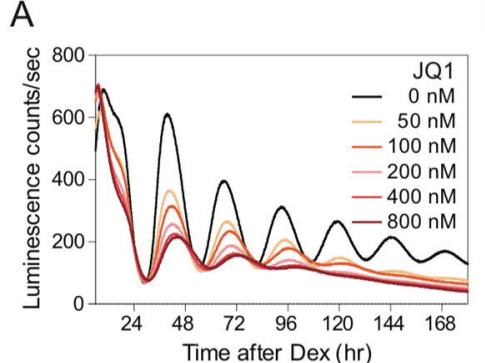

$\mathrm{D}$
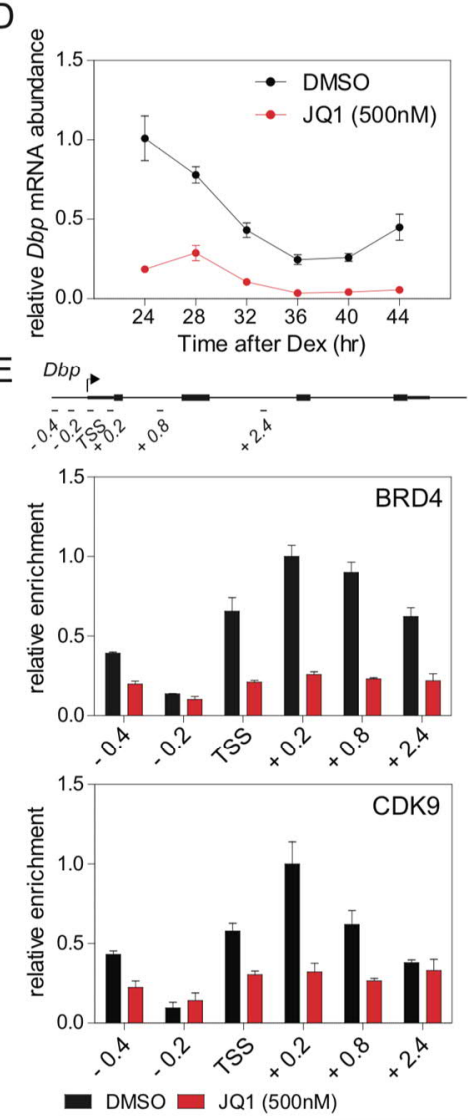

B

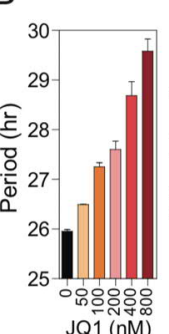

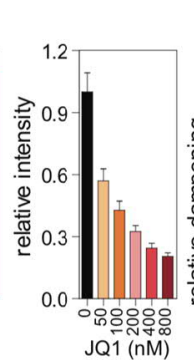

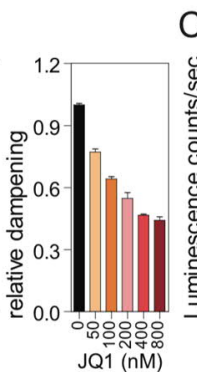

C

F Dbp
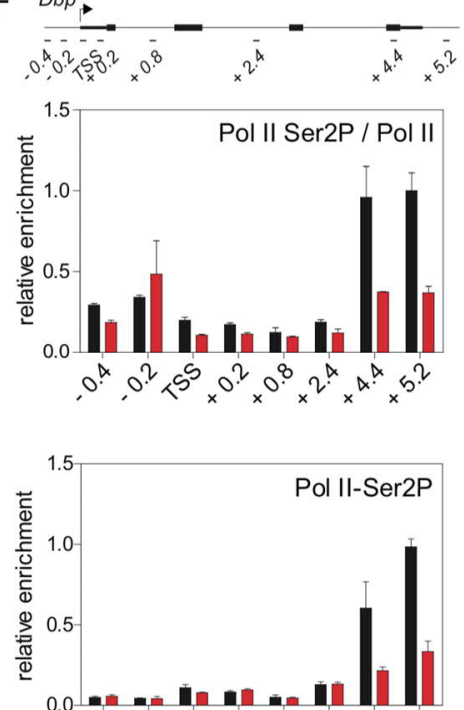

$0^{x}+0^{2} \times 5^{5} \times 0^{2} \times 0^{8} \times 2^{2} \times 0^{x} \times 0^{2}$

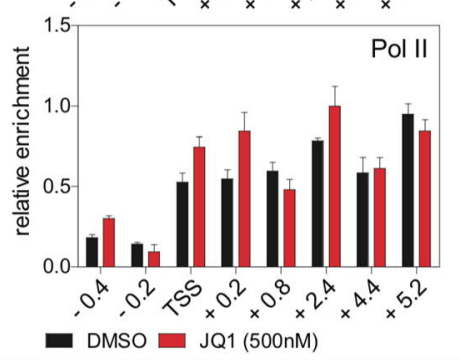

Figure 12: BRD4 Controls Clock Gene Expression.

(A) Bioluminescence recordings of dexamethasone (Dex) synchronized Bmall-LUC fibroblasts treated with increasing concentrations of JQ1 $(n=4)$. (B) Change of period, luminescence intensity, and damping rate relative to DMSO control $(n=4)$. (C) Bioluminescence recording of Dex-synchronized Bmall-LUC fibroblasts before and after wash out of $500 \mathrm{nM}$ JQ1 ( $=4)$. (D) Dbp mRNA dampening in Dex-synchronized fibroblasts by $500 \mathrm{nM}$ JQ1 ( $\mathrm{n}=3$; two-way ANOVA, see Table 10. (E and F) ChIP analysis of BRD4 and CDK9 (E) and Ser2-phosphorylated RNA Pol II (normalized to total RNA Pol II) (F) for the Dbp gene carried out 24 hours after Dex synchronization of the cells $(\mathrm{n}=3)$. Schematic diagrams show the genomic structure of the $D b p$ locus. Horizontal lines represent PCR-amplified genomic regions. All data are shown as mean \pm SEM. Note that $D b p$ has several additional intronic RNA Pol II pausing sites (Sobel et al., 2017). For validation of ChIP-grade quality of antibodies see Figure 10. 
Pol II pause release was suppressed (Harlen and Churchman, 2017). Reduced Dbp expression could be due to lower levels of BMAL1 and not abrogated BRD4-P-TEFb recruitment. To show that this is not the case, constitutively ectopic $C M V$ promoter-driven Bmal1-myc was expressed in BMAL1-deficient cells. BRD4 inhibition by JQ1 strongly reduced endogenous expression levels of $D b p$ mRNA but had no effect on mRNA or protein levels of Bmall-myc (Figure 13A). Furthermore, BRD4 inhibition neither affected endogenous mRNA nor protein levels of Bmall (Figure 13B) in Bmall-LUC fibroblasts at 24 hours after Dex synchronization. Finally, the binding of BMAL1 and of acetylated BMAL1 (AcBMAL1) protein to the Dbp promoter was not affected by BRD4 inhibition in these cells (Figure 13C). Together, our inhibitor studies suggest that BRD4-P-TEFb is recruited to the $E$-box-containing clock-controlled gene $D b p$, which subsequently allows RNA Pol II pause release and transcription elongation. Thus, for this circadian clockcontrolled gene, RNA Pol II pause release may serve as an important regulatory step of its transcription.
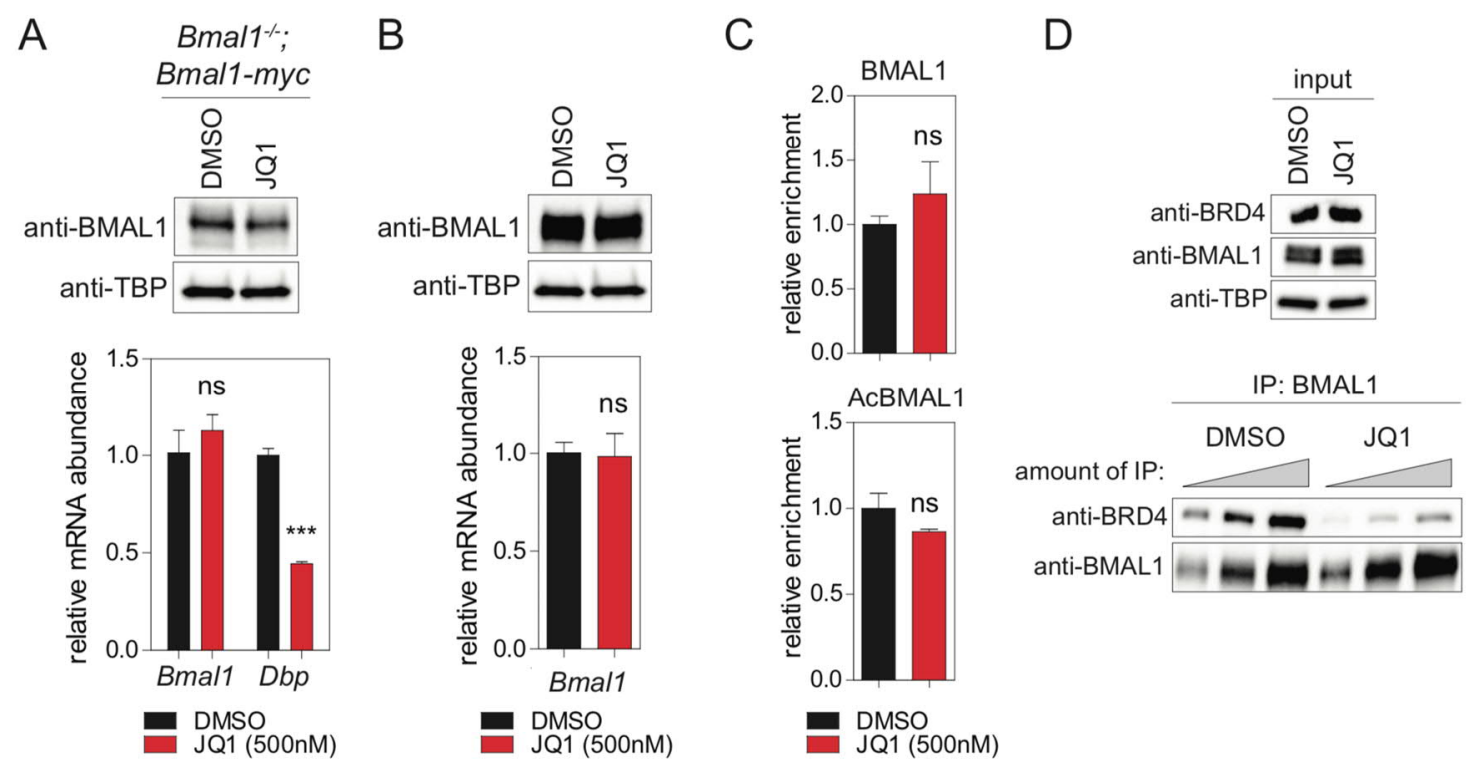

Figure 13: BRD4 Inhibition Does Not Directly Affect Bmal1 Expression.

(A) Immunoblot and mRNA expression analysis of Dex-synchronized (24 hours after) Bmal1 $^{-/}$MEFs transfected with empty vector or Bmall-myc treated with either DMSO or JQ1 $(500 \mathrm{nM})(\mathrm{n}=3)$. (B) BMAL1 protein abundance and Bmal1 mRNA expression in DMSO or JQ1 $(500 \mathrm{nM})$ treated cells isolated 24 hours after Dex synchronization $(\mathrm{n}=3)$. (C) ChIP analysis of BMAL1 and acetylated BMAL1 for the Dbp gene carried out $24 \mathrm{~h}$ after Dex synchronization of MEFs (n=3). (D) Interaction of BMAL1 with BRD4 in fibroblasts treated with either DMSO or JQ1 $(500 \mathrm{nM})$. Immunoblots show results with increasing equivalents of IPs (two-fold dilutions) from nuclear extracts isolated from cells 24 hours after Dex synchronization. All data are shown as mean $\pm \operatorname{SEM}(n=3 ; n s>0.05$ and $* * * p<0.001$, Student's t-test). 


\subsection{Acetylation of BMAL1 at Lysine $\mathbf{5 3 8}$ is Required to Initiate Circadian Productive Transcription Elongation}

BRD4 binds to acetylated lysines of transcription factors/histones at the promoter of paused genes (Shi and Vakoc, 2014). The observed strong effect of JQ1 on the cellular circadian rhythm raises the possibility that among BRD4 targets there are core clock components, such as BMAL1, which is acetylated at Lys538 (Hirayama et al., 2007) and could therefore provide a binding site for BRD4. This particular lysine was mutated to an arginine (BMAL1 ${ }^{\mathrm{K} 538 \mathrm{R}}$ ) using the CRISPR/Cas9 technique (Figure 8 and Figure 14A and B). BMAL1 ${ }^{\mathrm{K} 538 \mathrm{R}}$ protein was as stable as wildtype BMAL1 (Figure 15A), and subcellular localization and phosphorylation status of both forms were similar (Hirayama et al., 2007). Enrichment of both BMAL1 variants to the promoters of the Dbp, Perl, and RevErbo genes was also found to be unchanged (Figure 14C). Next, the interaction of BRD4 and CDK9 with BMAL1 was examined in control fibroblasts and fibroblasts containing engineered BMAL1 ${ }^{\mathrm{K} 538 \mathrm{R}}$. Immunoprecipitation experiments showed that in BMAL1 ${ }^{\mathrm{K} 538 \mathrm{R}}$ expressing cells this interaction was markedly reduced (Figure 15A). When wildtype cells were JQ1-treated, this interaction was substantially dampened (Figure 13D). ChIP experiments demonstrated that BMAL1 ${ }^{\mathrm{K} 538 \mathrm{R}}$ mutant cells showed a diminished recruitment of BRD4 and of the P-TEFb subunit CDK9 to the TSS of Dbp, Perl, and Rev-Erb $\alpha$ (Figure 15B). In addition, enrichment of Ser2-phosphorylated RNA Pol II was markedly reduced (Figure 15B). Since both, control fibroblasts and BMAL1 ${ }^{\text {K538R }}$ mutant cells stably expressed a clock-driven luciferase reporter, the effect of the K538R mutation on luminescence rhythms was assessed. In BMAL1 ${ }^{\mathrm{K} 538 \mathrm{R}}$ cells virtually no rhythmic luciferase reporter expression was recorded for any of the 3 clones (Figure 16A). In addition, in these cells there was a pronounced reduction of peak expression of endogenous Dbp, Perl, and Rev-Erba mRNAs (Figure 16B-D). It is possible that BMAL1 acetylation could additionally promote transcription initiation. However, the occupancy of the general transcription factor TFIIE $\alpha$ (part of the RNA Pol II transcription initiation complex (Sainsbury et al., 2015)) at the TSS of Dbp, Perl, and Rev-Erbo genes was not affected in mutant cells (Figure 17). This favors the idea that transcription elongation, and not initiation, is the process that is primarily regulated by acetylation of BMAL1. These data support a mechanism in which acetylated BMAL1 recruits BRD4 and $\mathrm{P}-\mathrm{TEFb}$ to $E$-box-containing circadian clock genes which then results in a release of RNA Pol II from its paused state, thereby allowing productive elongation. 


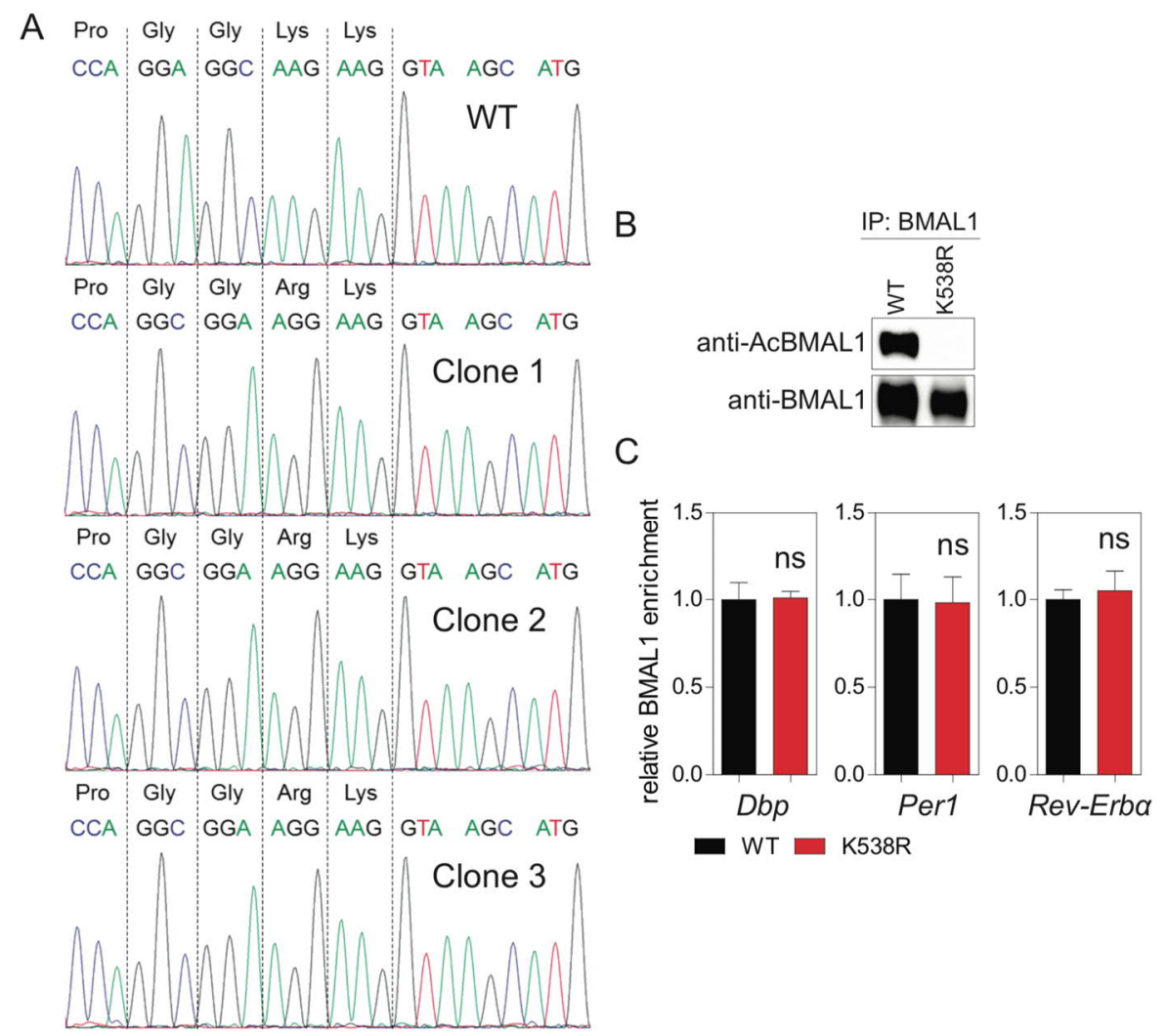

Figure 14: CRISPR/Cas9-mediated Generation and Characterization of BMAL1 ${ }^{\text {K538R }}$ Mutant Cells.

(A) Schematic representation of the gene targeting strategy for generating BMAL1 ${ }^{\mathrm{K} 538 \mathrm{R}}$ mutant cells using the CRISPR/Cas9 system. The gRNA-targeting sequence is underlined and the PAM sequence is indicated in red. The oligonucleotide donor (120 bp) is shown below the targeted site, with the Lys-to-Arg substitution indicated in red. Silent point mutations were introduced for the two adjacent Gly to prevent cutting of the donor DNA or re-cutting of the genome after homology-directed recombination (shown in red). (B) Representative sequencing results from PCR amplicons of the Lys538 region from three individual clones that were subsequently used for experimental work. Primer sequences are specified in Table 11. (C) BMAL1 IPs from wildtype or BMAL1 ${ }^{\mathrm{K} 538 \mathrm{R}}$ fibroblast nuclear extracts carried out 24 hours after Dex synchronization of fibroblasts were immunoblotted with anti-AcBMAL1 and anti-BMAL1 antibodies. (D) ChIP analysis of BMAL1 binding to Dbp, Perl, and Rev-Erbo genes in wildtype and BMAL1 ${ }^{\text {K538R }}$ synchronized fibroblasts 24 hours after Dex synchronization. Data are shown as mean \pm SEM ( $=3 ; n s>0.05$ Student's t-test). 


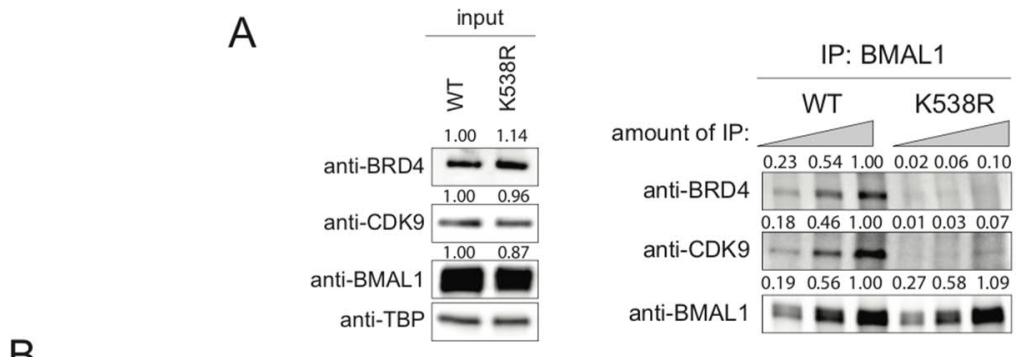

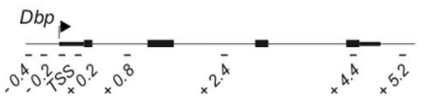

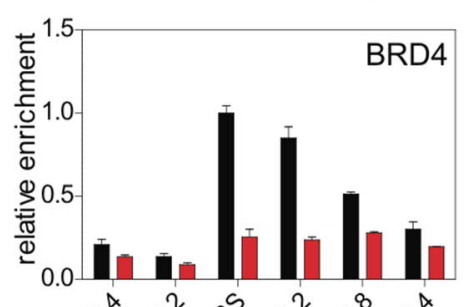

$0^{x}, 0^{2} \times 5^{5} \times 0^{2} \times 0^{8} \times 2^{x}$
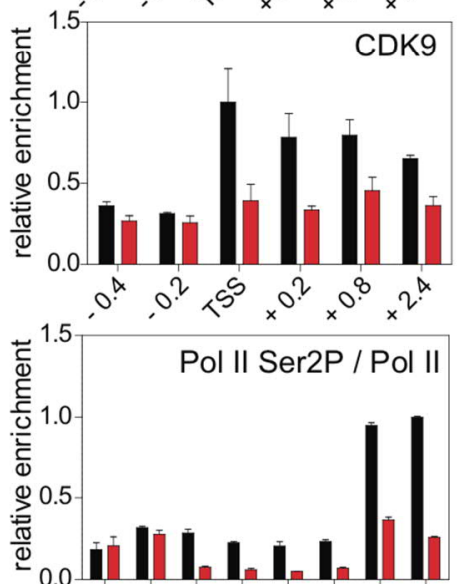

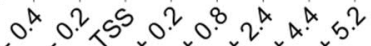
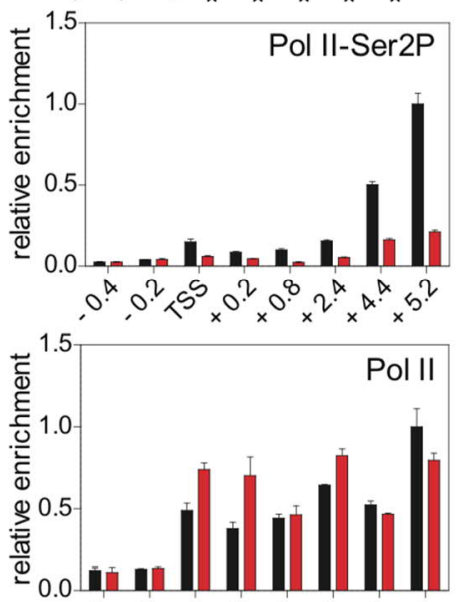

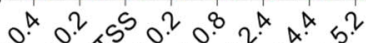

- WT $\square 538 \mathrm{R}$
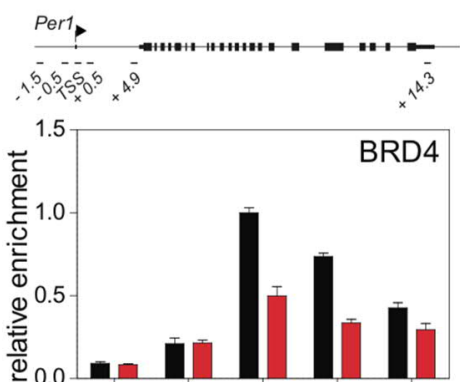

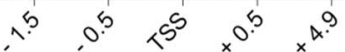
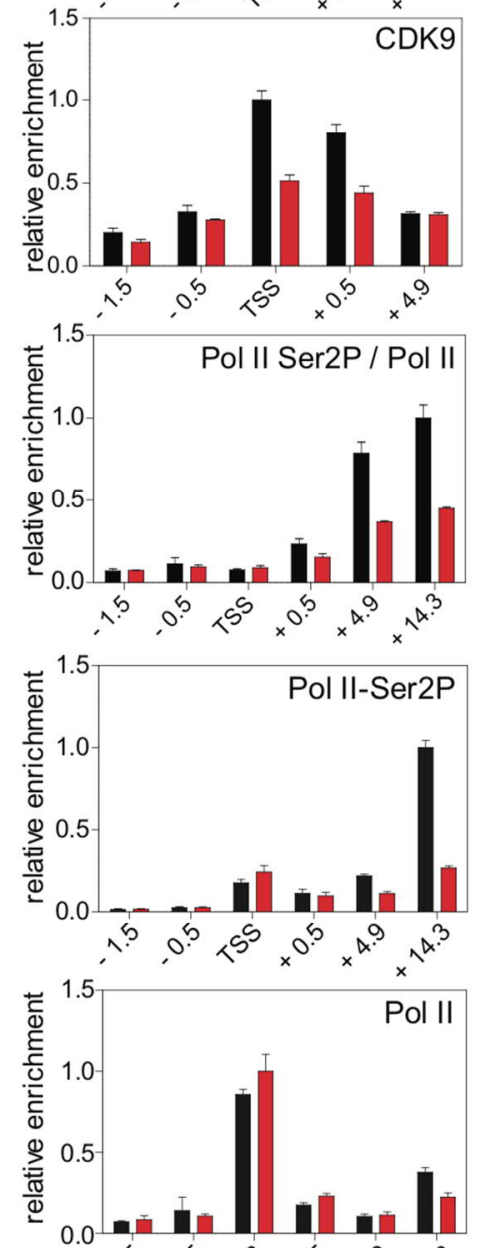

$x^{5} 0^{5}<^{5} \times x^{50} \times x^{9.9} \times x^{3^{3}}$
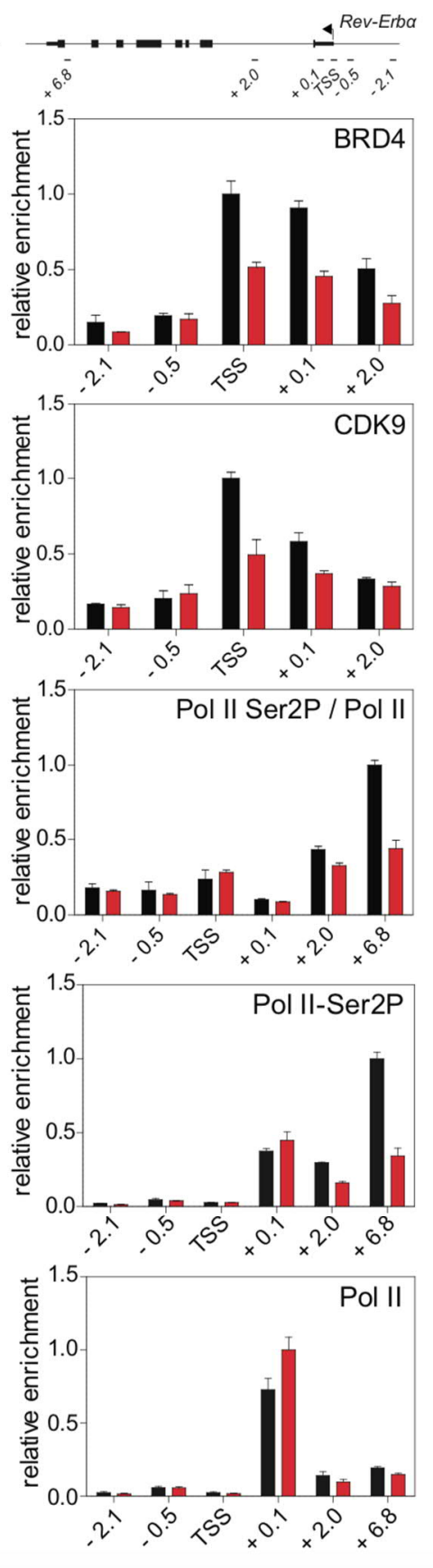

Figure 15: Lys538 Acetylation of BMAL1 Is Essential for Transcription Elongation. (A) Interaction of BMAL1 or BMAL1 ${ }^{\mathrm{K} 538 \mathrm{R}}$ with BRD4 and CDK9 as shown by immunoblotting of BMAL1 IPs from nuclear extracts of synchronized fibroblast 24 hours after Dex treatment (two-fold dilutions). TBP (TATA-binding protein) was used as loading control. Numerical values represent intensities of chemiluminescence signals of 
individual bands, normalized to wildtype and loading control for input samples. (B) ChIP analysis of BRD4, CDK9 and Ser2P-RNA Pol II (normalized to total RNA Pol II) binding to the Dbp, Perl, and Rev-Erbo genes in wildtype and BMAL1 ${ }^{\mathrm{K} 538 \mathrm{R}}$ synchronized fibroblasts as shown in (A). Data are shown as mean $\pm \operatorname{SEM}(n=3)$.
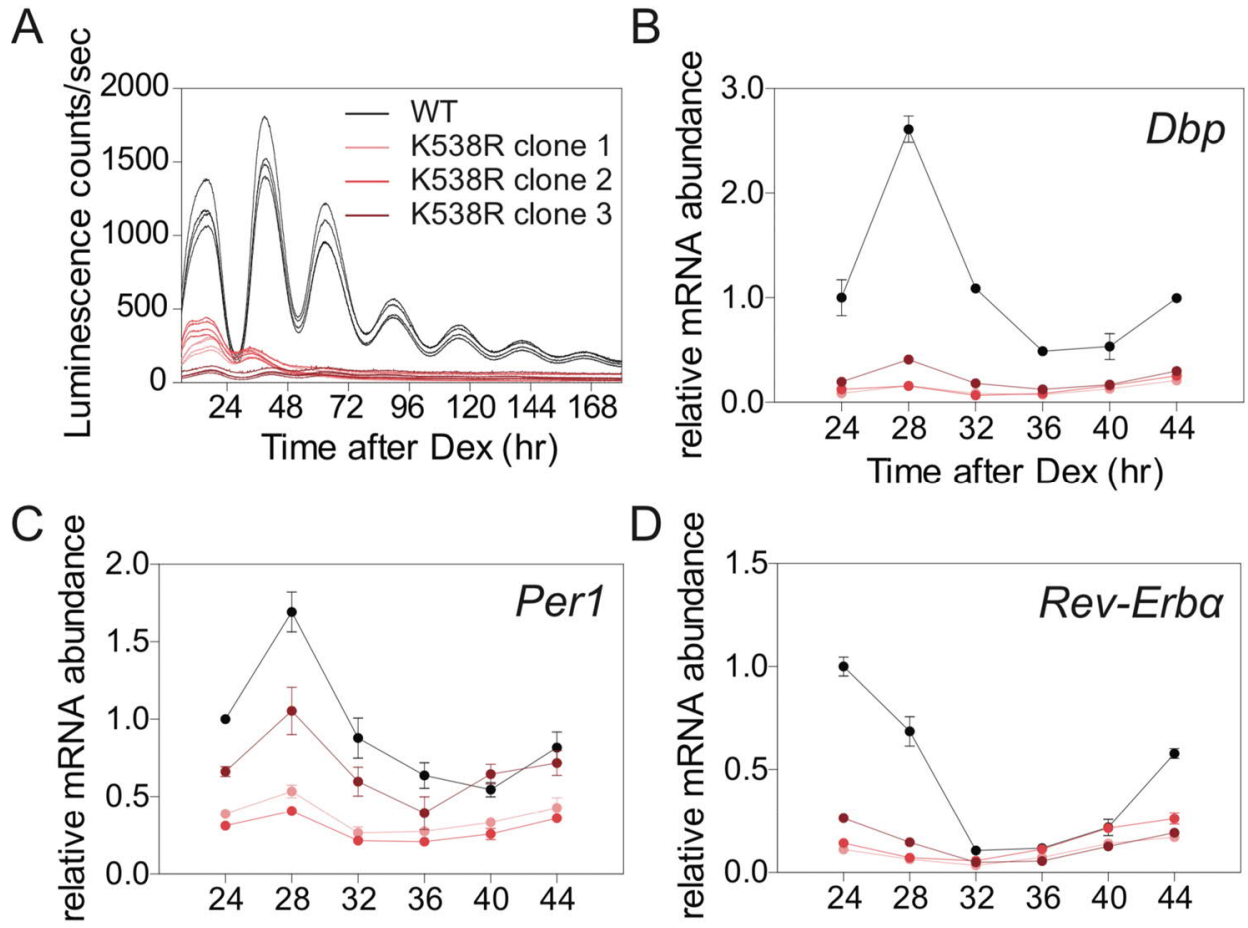

Figure 16: Lys538 Acetylation of BMAL1 Controls Clock Gene Expression.

(A) Bmal1-LUC bioluminescence tracings of synchronized wildtype fibroblasts and of three independent BMAL1 ${ }^{\mathrm{K} 538 \mathrm{R}}$ clones (n=4). (B - D) Dbp (B), Perl (C), and Rev-Erbo (D) mRNA expression analysis of wildtype or BMAL1 $\mathrm{K}^{538 \mathrm{R}}$ fibroblasts taken from the experiment shown in (A) $(n=3$, two-way ANOVA, see Table 12). All data are shown as mean \pm SEM.
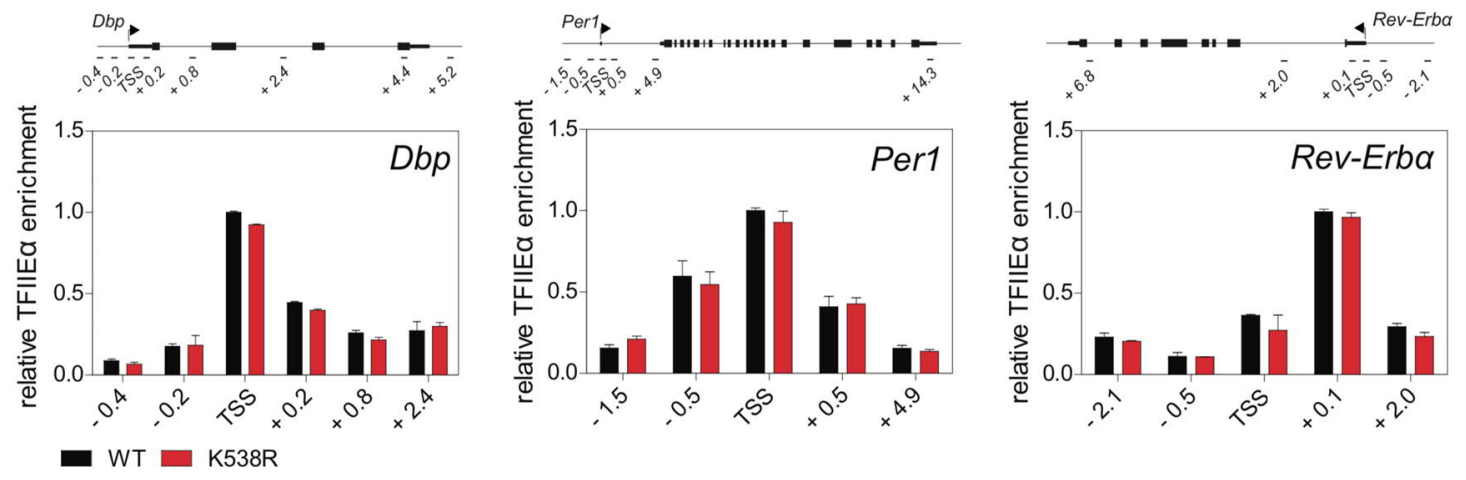

Figure 17: Acetylation of BMAL1 Is Unlikely to Affect Transcription Initiation.

ChIP analysis of TFIIE $\alpha$ binding to Dbp, Perl, and Rev-Erbo genes in wildtype and $\mathrm{BMAL1}^{\mathrm{K} 538 \mathrm{R}}$ synchronized fibroblasts. Data are shown as mean $\pm \operatorname{SEM}(\mathrm{n}=3)$. 


\subsection{TIP60 Acetylates BMAL1 at Lysine 538}

BMAL1 acetylation was reported to be carried out by CLOCK (Hirayama et al., 2007). However, in CLOCK-deficient fibroblasts, BMAL1 was acetylated at Lys538 to a similar extent as observed in wildtype cells (Figure 18A). CLOCK ${ }^{\text {mutA }}$ fails to acetylate histones H3 and H4 (Doi et al., 2006) and shows greatly diminished activity in the acetylation of BMAL1 (Hirayama et al., 2007). Both wildtype and mutant CLOCK showed a minimal increase of BMAL1 acetylation compared to a control sample (Figure 18B). Importantly, BMAL1 acetylation levels in the presence of CLOCK or CLOCK ${ }^{\text {mutA }}$ were indistinguishable. Together, these data suggest the existence of alternative acetyltransferases that catalyze BMAL1 acetylation. A candidate is TIP60 that co-purifies with CLOCK-BMAL1 (Doi et al., 2006) and acetylates a broad range of transcription factors (Ghobashi and Kamel, 2018; Judes et al., 2015). Co-transfection of BMAL1 with TIP60, but not enzymatically inactive TIP60 ${ }^{\mathrm{C} 369 \mathrm{~A} ; \mathrm{E} 403 \mathrm{Q}}$ (Yang et al., 2012), strongly enhanced Lys538 BMAL1 acetylation regardless of the presence of wildtype or mutant CLOCK (Figure 18B). Lys538 acetylation of BMAL1 by TIP60 occurred in a dosedependent manner and acetyltransferase-deficient TIP60 ${ }^{\mathrm{C} 369 \mathrm{~A} ; \mathrm{E} 403 \mathrm{Q}}$, unlike CLOCK $^{\text {mutA }}$, led to baseline acetylation levels of BMAL1 similar to mock transfected cells (Figure 18C). Lys538 appeared to be the predominant lysine acetylated by TIP60 as the signal obtained with an anti-pan-AcK antibody was strongly diminished in cells co-transfected with BMAL1 ${ }^{\text {K538R }}$ and TIP60 (Figure 18C). Recombinant TIP60 ${ }^{\mathrm{GST}}$ acetylated recombinant BMAL1 ${ }^{\mathrm{GST}}$ at Lys538 (Figure 18D). TIP60 is a nuclear, chromatinassociated protein (Figure 18E and F) and co-precipitated with wildtype and BMAL1 ${ }^{\text {K538R }}$ (Figure 18G) suggesting an interaction between the two proteins directly on the chromatin that is not influenced by acetylation of BMAL1. Both, BMAL1 and TIP60 ${ }^{\mathrm{V} 5}$ proteins bound to the E-boxes of the $D b p$ gene (Figure 18H) further supporting the idea that interaction between the two proteins is happening on the DNA. BMAL1 was also the only E-box associated clock protein (Koike et al., 2012) that was acetylated in a TIP60 activitydependent manner (Figure 19A). Although, PER2 was as well found to be acetylated (Asher et al., 2008), this acetylation was not dependent on TIP60 (Figure 19B). Neither CRY1, CRY2 nor CLOCK were acetylated (Figure 19A, C and D). Collectively, these data provide strong evidence that BMAL1 is a direct substrate of TIP60. 
A

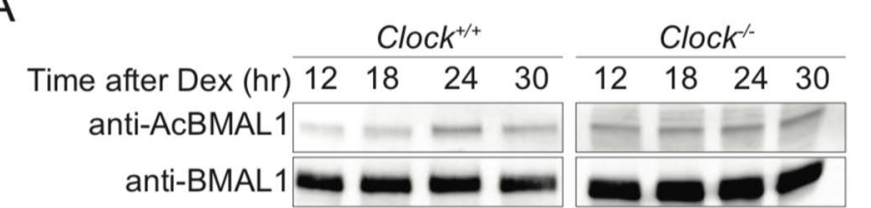

B

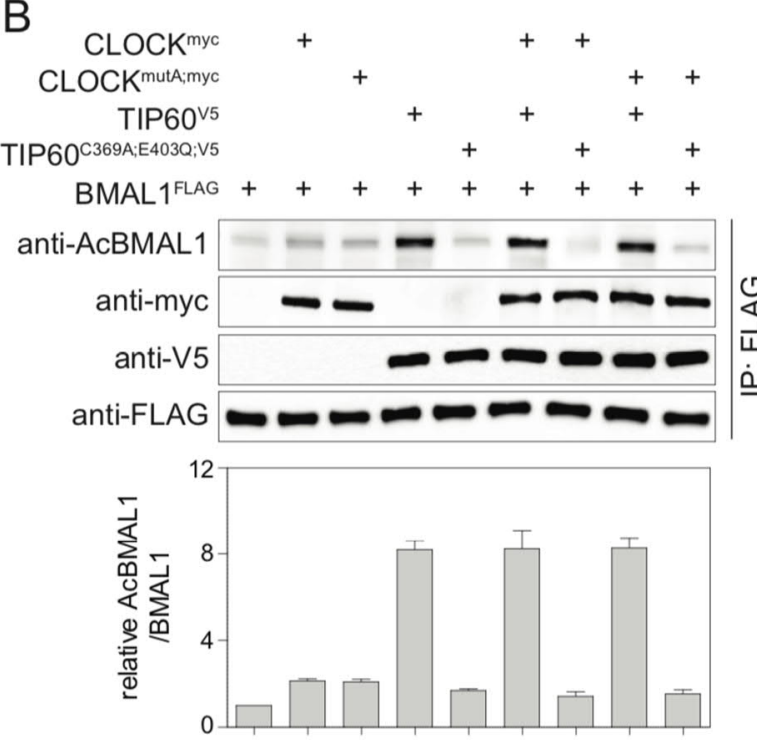

E

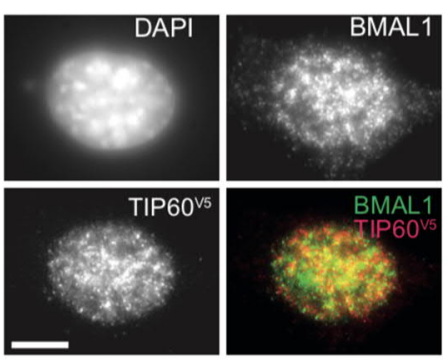

F

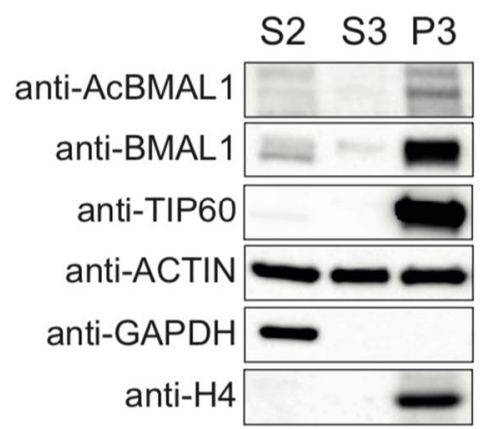

C

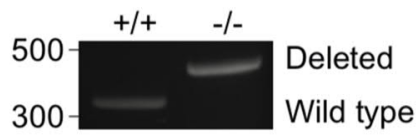

$$
\text { TIP60 }
$$

TIP60 $0^{\text {V }} \mathrm{ng}:$

$\begin{array}{llllll}1 & 2 & 3 & 4 & 5 & 6\end{array}$

$2505001000 \quad 1000$

T69A:E4030; 55 ng:

BMAL1 $^{\text {myc }}$ ng: 10001000100010001000

BMAL ${ }^{\text {K538R;myc }} \mathrm{ng}$ :

anti-AcBMAL1

anti-pan-AcK

anti-myc

anti-V5

anti-myc

anti-V5

1000
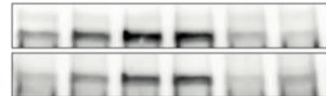

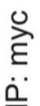

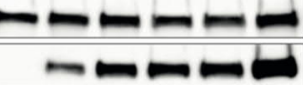

D

BMAL1GST
TIP60 $^{\text {GST }}++$
ACCOA

AcCoA

anti-pan-AcK

anti-AcBMAL1

anti-GST

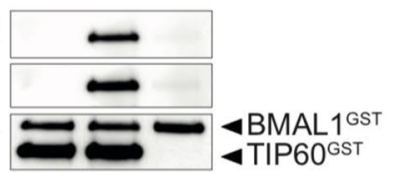

G

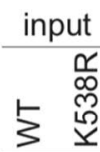

IP: BMAL1

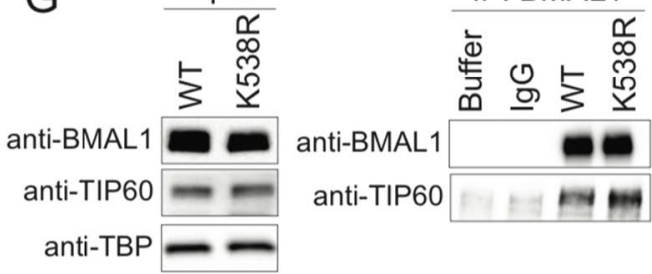

$\mathrm{H}$

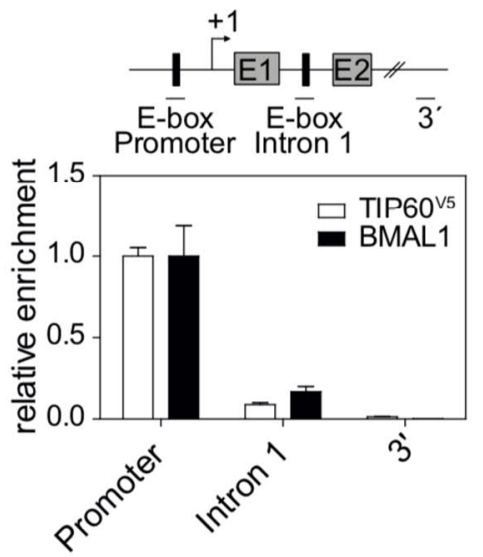

Figure 18: TIP60 Acetylates BMAL1.

(A) BMAL1 IP analysis of control and Clock-deficient MEF nuclear extracts over a 24hour time course. For both genotypes, equivalent amounts of BMAL1 were included as shown in the anti-BMAL1 probed Western blot. Lys538 acetylation of BMAL1 was detected using an anti-AcBMAL1 antibody (left). Wildtype and mutant cells were PCRgenotyped to confirm their genotype (right) (DeBruyne et al., 2006). (B and C)

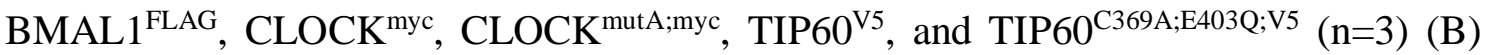
and BMAL1 ${ }^{\text {myc }}$, BMAL1 ${ }^{\text {K538R,myc }}$, TIP60 ${ }^{\mathrm{V} 5}$, and TIP60 ${ }^{\mathrm{C} 369 \mathrm{~A} ; \mathrm{E} 403 \mathrm{Q} ; \mathrm{V} 5}$ (C) were transiently 
overexpressed in HEK293T cells in the combinations indicated. Lysates were subjected to IPs and immunoblotted with antibodies indicated. (D) Recombinant BMAL1 ${ }^{\text {GST }}$, $\mathrm{TIP}^{\mathrm{GST}}$ and acetyl-CoA were incubated and the presence of acetylated BMAL1 was detected by immunoblotting using either a pan-acetyl-Lys or a Lys538-specific AcBMAL1 antibody. (E) Nuclear localization of TIP60 ${ }^{\mathrm{V} 5}$ and BMAL1. Tip60-1 MEFs stably expressing Tip60 $0^{V 5}$ were stained with an antibody against anti-V5 and anti-BMAL1 and counterstained with DAPI. Scale bar: $10 \mu \mathrm{m}$ (F) TIP60 and Lys538 acetylated BMAL1 colocalize to chromatin. Western blotting of cytoplasmic (S2), nucleoplasmic (S3) and chromatin (P3) protein fractions from Dex-synchronized MEFs (24 hours after Dex) using the antibodies indicated. H4 (chromatin) and GAPDH (cytoplasmic) antibodies were used to assess the purity of the fractions. (G) Immunoblot analysis of the interaction between endogenous TIP60 with BMAL1 or BMAL1 ${ }^{\text {K538R }}$. IPs from Dexsynchronized MEFs (24 hours after Dex) nuclear extracts were immunoblotted with the antibodies indicated. IgG and buffer (mock IP) served as negative controls. (H) ChIP analysis of Tip60 $0^{--}$; Tip60 ${ }^{V 5}$ MEFs using anti-V5 and anti-BMAL1 antibodies. The diagram shows the 5' region of the $D b p$ gene. E-boxes that bind CLOCK-BMAL1 at exons 1 (E1), exon 2 (E2), and transcription start sites are shown. Horizontal lines represent PCR-amplified genomic regions. The 3' UTR region was used as a control ( $\mathrm{n}=$ 3 ). All data are shown as mean \pm SEM.

A

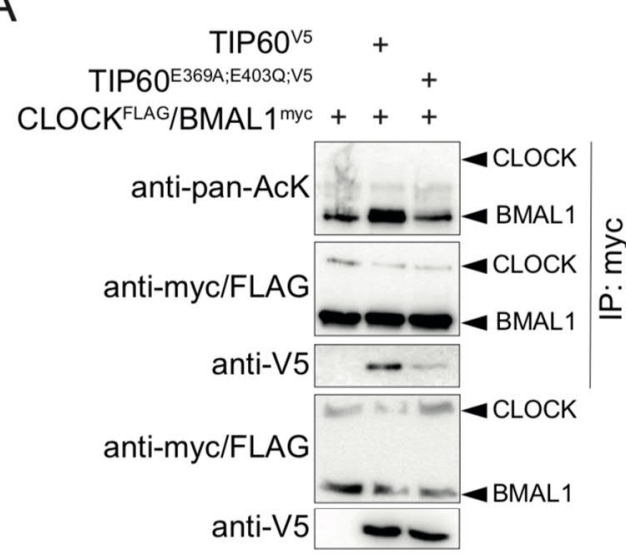

C

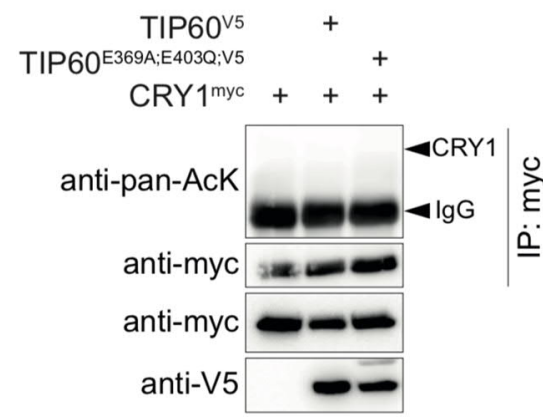

B

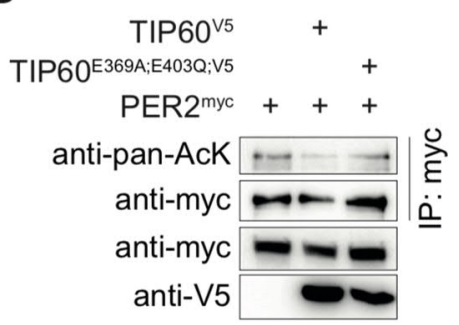

D

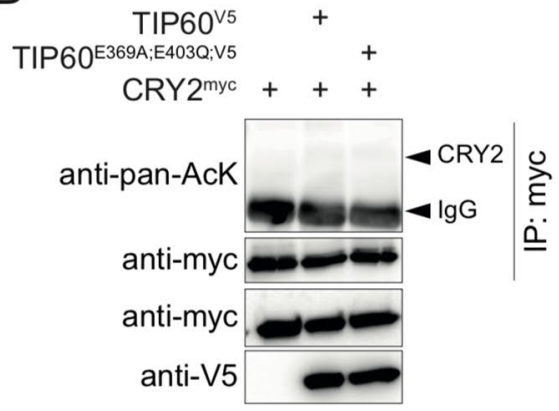

Figure 19: BMAL1 Is the Only Clock Protein Acetylated by TIP60.

(A - D) Screen for putative TIP60 substrates. TIP60 ${ }^{\mathrm{V} 5}$ or TIP60 ${ }^{\mathrm{C} 369 \mathrm{~A} ; \mathrm{E} 403 \mathrm{Q} ; \mathrm{V} 5}$ were coexpressed with putative TIP60 substrates $\mathrm{CLOCK}^{\text {myc }}$ and BMAL1 ${ }^{\text {myc }}$ (A), PER2 ${ }^{\text {myc }}$ (B), CRY1 ${ }^{\text {myc }}(C)$, and CRY2 ${ }^{\text {myc }}$ (D) in HEK293T cells. Acetylation of putative substrates was assessed using an anti-pan-AcK antibody. 


\subsection{TIP60 Is Essential for a Functional Circadian Clock in the Mouse}

This unexpected discovery of a new potential regulator of the circadian clock prompted our laboratory to generate conditional TIP60 mice to assess the in vivo function of this enzyme and provide fibroblasts that were used to study the molecular effect of TIP60 on the clock in vitro. Tip60fl/- mice carrying one conditional and one deleted allele of Tip60 (Figure 7) were mated to Syt10-CRE driver mice. This driver is strongly active in the postmitotic neurons of the SCN (Husse et al., 2011) circumventing cell lethality associated with TIP60-deficiency (Hu et al., 2009). All offspring carrying two copies of the knock-in Cre allele (Syt10 $\left.{ }^{\mathrm{Cre} / \mathrm{Cre}} \mathrm{Tip} 6 \mathrm{O}^{\mathrm{fl} / \mathrm{-}}\right)$ and half of the mice carrying one copy of the knock-in Cre allele ( $\mathrm{Syt} 10^{\mathrm{Cre} /+} \mathrm{Tip} 6 \mathrm{O}^{\mathrm{fl} / \text { - }}$ ) became immediately arrhythmic upon release into constant darkness (DD) (Figure 20A and B). The rest of the Syt $10^{\mathrm{Cre} /+}$ Tip $60^{\mathrm{fl} / \text { - mice }}$ showed either longer $(29 \%)$ or normal $(21 \%)$ free-running periods. Such a Cre dosage dependence was also observed with Syt10-CRE-mediated deletion of Bmall ${ }^{f l f l}$ and, therefore, characterizes this particular driver (Husse et al., 2011). Immunohistochemical staining for TIP60 in the SCN showed nuclear signal, which was absent in arrhythmic Syt10 ${ }^{\text {Cre/+ }}$ Tip60 $0^{\text {fl- }}$ mice (Figure 20C). Moreover, circadian expression patterns of Perl, Dbp, and Bmall in the SCN were severely dampened (Figure 20D) in line with the behavioral arrhythmicity observed in these animals. DAPI staining of SCN sections from Syt $10^{\mathrm{Cre} /+} \mathrm{Tip}^{\mathrm{O}} 6 \mathrm{O}^{+/+}$and arrhythmic Syt $10^{\mathrm{Cre} /+}$ Tip $60^{\mathrm{fl} / \mathrm{-}}$ mice showed no obvious changes in SCN morphology or in the number of SCN cells (control: $827 \pm 60$ cells per section; mutant: $850 \pm 68$ cells per section). In addition, no TUNEL signal, indicating apoptotic cells, could be detected in the SCN of TIP60-deficient animals (Figure 20E). Together, these experiments indicate that SCN cells were fully viable after deletion of Tip60.

The essential circadian function of TIP60 was also seen in MEFs. Immortalized MEFs were prepared from Tip60 fl/- mouse embryos and were transduced with a Bmall-LUC reporter. Confluent, i.e. non-dividing MEFs, were infected with AdCre to delete TIP60. Cell confluency is sufficient to induce cell cycle quiescence (Hayes et al., 2005), thus bypassing the role of TIP60 in cell cycle regulation (Sykes et al., 2006; Tang et al., 2006). In TIP60-deficient MEFs, Lys538 acetylation of endogenous BMAL1 was markedly reduced (Figure 21A and B), illustrating the crucial role of TIP60 in BMAL1 acetylation. When endogenous TIP60 was replaced by TIP60 ${ }^{\mathrm{C} 369 \mathrm{~A} ; \mathrm{E} 403 \mathrm{Q}}$ in the Bmall-LUC reporter cell line, luciferase rhythms and mRNA rhythms of endogenous $D b p$ were abolished 
A

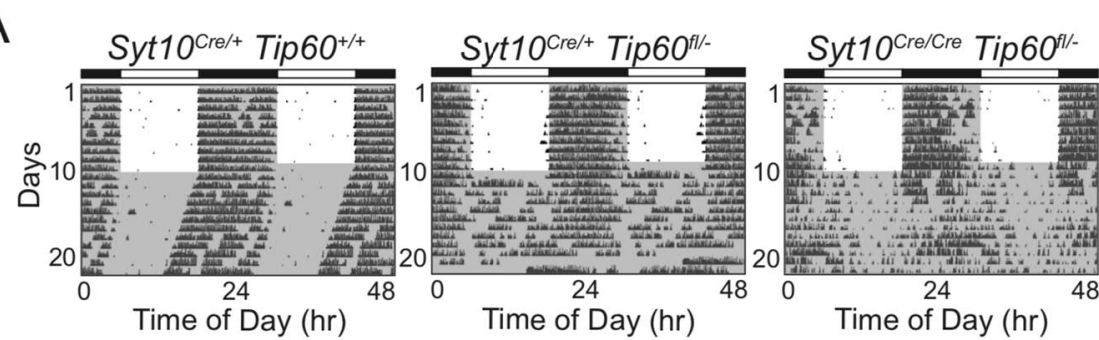

B

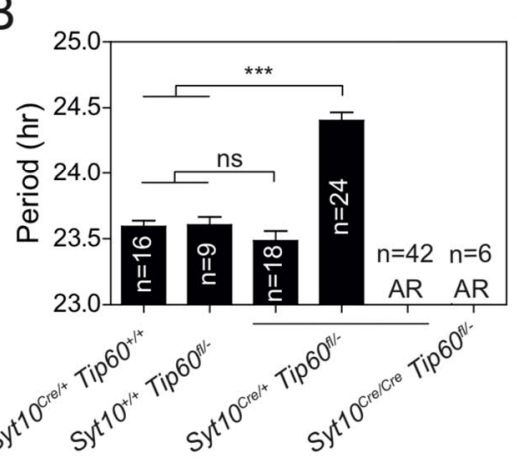

C

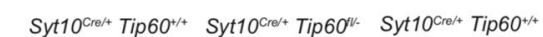
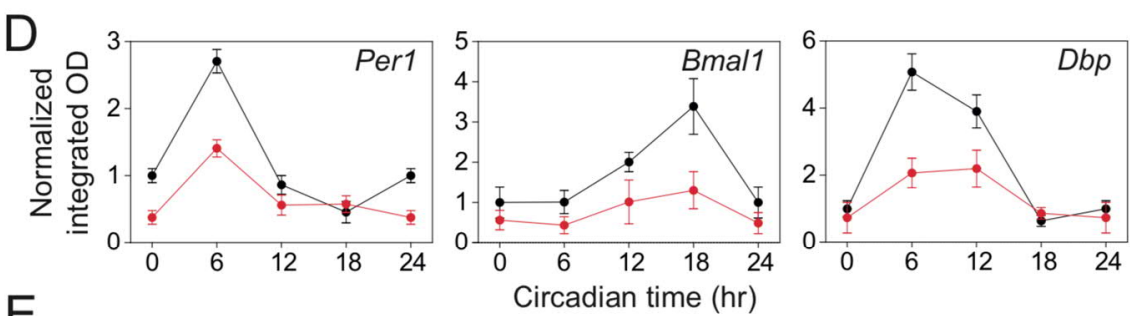

E

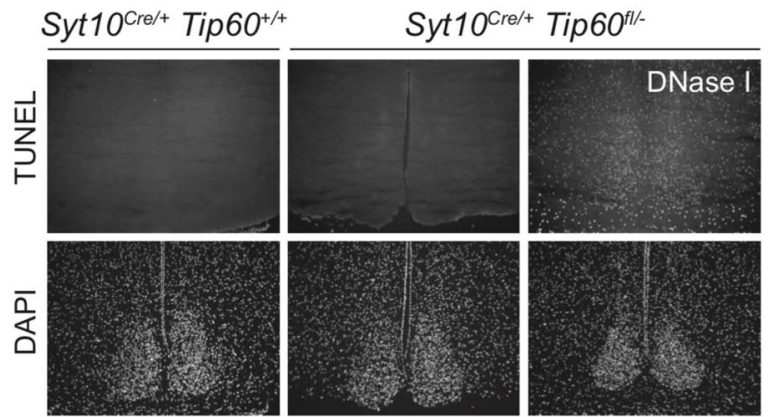

Figure 20: TIP60-deficiency Evokes a Circadian Phenotype in Mice and Disrupts Rhythmic Clock Gene Expression in the SCN.

(A) Double-plotted actograms of control $\left(\mathrm{Syt} 10^{\mathrm{Cr} /+} \mathrm{Tip} 60^{+/+}\right)$and mutant $\left(\mathrm{Syt} 10^{\mathrm{Cre} /+}\right.$ Tip60 $0^{f /-}$, Syt $10^{\mathrm{Cre} / \mathrm{Cre}}$ Tip $60^{f / /}$ ) mice under 12-hour/12-hour light-dark and constant darkness conditions. Grey shadings indicate dark phases. (B) Free-running periods determined by $\chi^{2}$ periodogram analysis. AR: arrhythmic $(* * * p<0.001$, one-way ANOVA with Bonferroni post-test). (C) TIP60-immunoreactivity (ir) in the SCN of

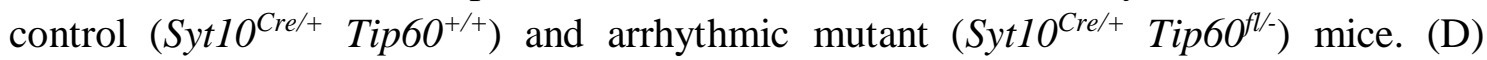
Densitometric quantification of clock gene mRNA expression from radioactive in situ hybridization analysis of control (Syt10 ${ }^{\mathrm{Cr} /+} \mathrm{Tip}^{+O^{+/+}}$; black) and mutant (Syt10 $\mathrm{Cre/+}$ Tip $60^{f / /}$; red) SCN sections ( $=3$; two-way ANOVA, see Table 13). (E) TUNEL immunoreactivity on brain sections of control (Syt10 $\left.{ }^{\mathrm{Cre} /+} \mathrm{Tip}^{+/+}\right)$, arrhythmic $\left(\right.$ Syt $10^{\mathrm{Cre} /+}$ Tip $\left.6 \mathrm{f}^{f / /-}\right)$ animals, and mutant $\left(\right.$ Syt $10^{\mathrm{Cre} /+}$ Tip $\left.60^{f / /-}\right)$ sections treated in situ with DNase I. 
A

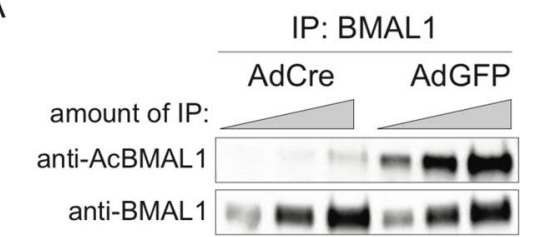

B

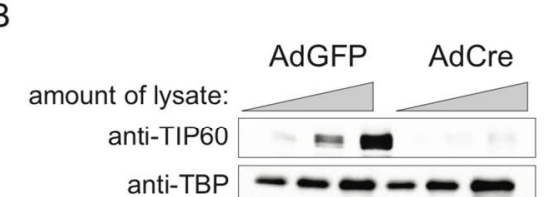

E

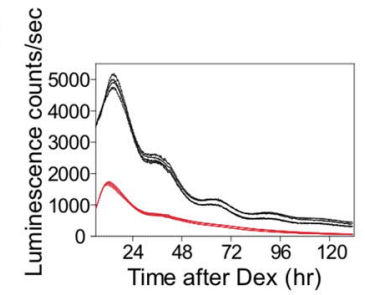

$\mathrm{G}$

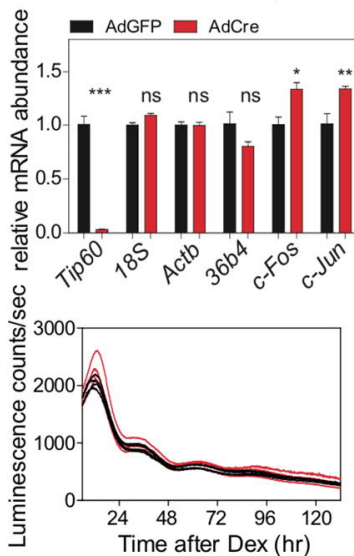

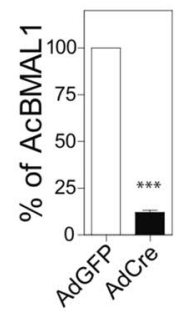

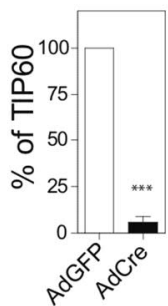

C

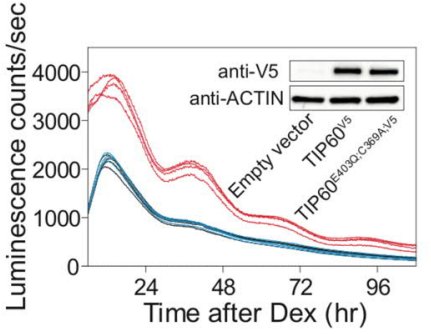

D

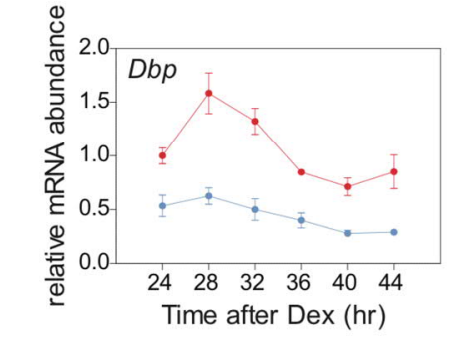

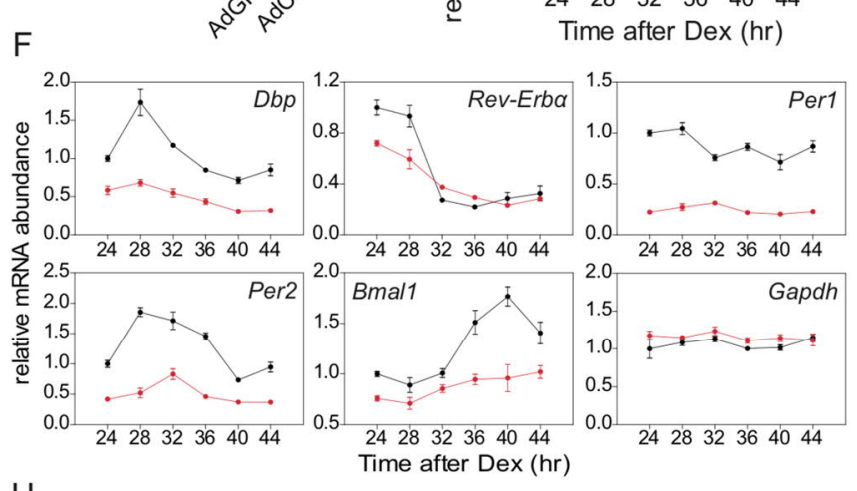

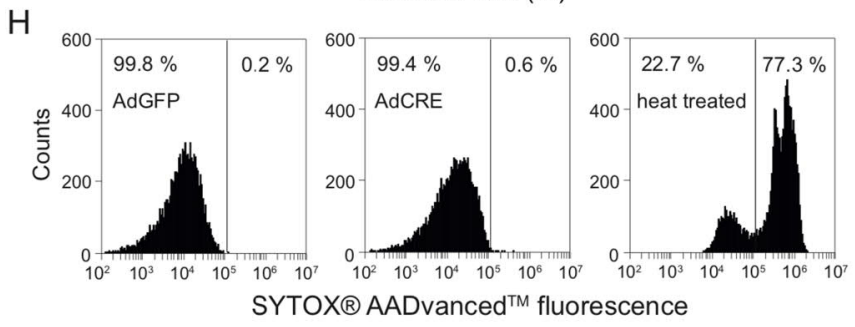

Figure 21: TIP60-deficiency Disrupts Rhythmic Clock Gene Expression in MEFs. (A and B) IPs from nuclear extracts (A) and nuclear extracts (B) from AdGFP (control) and AdCre (mutant) transduced unsynchronized Tip60 $0^{f /-}$ MEFs (two-fold dilutions) were immunoblotted with antibodies indicated and signal intensities were quantified and normalized (n=3). (C) Tip60 fl-; Bmall-LUC MEFs stably expressing TIP60 ${ }^{\mathrm{V} 5}$ (red), TIP60 ${ }^{\mathrm{C} 369 \mathrm{~A} ; \mathrm{E} 403 \mathrm{Q} ; \mathrm{V} 5}$ (blue) or empty vector (black, coinciding with the blue tracing) were transduced with AdCre and bioluminescence was recorded ( $\mathrm{n}=4)$. (D) $D b p$ expression profiles in TIP60 ${ }^{\mathrm{V} 5}$ and TIP60 ${ }^{\mathrm{C} 369 \mathrm{~A} ; \mathrm{E} 403 \mathrm{Q} ; \mathrm{V} 5}$ cells shown in $(\mathrm{C}) .(\mathrm{n}=3$; two-way ANOVA, see Table 14). (D) Bioluminescence recordings of synchronized Tip60 fl/- Bmal1-LUC MEFs transduced with AdGFP (black) or AdCre (red) $(n=4)$. (F) mRNA expression analysis of Dex-synchronized Tip60 $0^{f /-}$ MEFs transduced with either AdGFP (black) or AdCre (red) ( $\mathrm{n}=3$; two-way ANOVA, see Table 15). (G) mRNA expression of housekeeping and immediate early genes in Tip $60^{f /-}$ MEFs transduced with either AdGFP or AdCre, 24 hours after Dex synchronization $(\mathrm{n}=3$; $\mathrm{ns}>0.05$ and ***p $<0.001$, Student's t-test). (H) Flow cytometric analysis of AdGFP, AdCre transduced, and heattreated $\left(3 \mathrm{~min}, 95^{\circ} \mathrm{C}\right)$ confluent Tip $60^{f l-}$ MEFs. (I) Bioluminescence tracing of Dexsynchronized Bmall-LUC MEFs transduced with AdGFP (black) or AdCre (red) (n=4). All data are shown as mean \pm SEM. 
(Figure 21C and D). Deletion of Tip60 also resulted in a major dampening of the rhythmic expression of the luciferase reporter (Figure 21E) and exhibited a strongly reduced expression of endogenous clock genes (Figure 21F) but not of several housekeeping genes (Figure $21 \mathrm{~F}$ and G). Even the expression of $c$-Fos and $c$-Jun, two immediate-early genes, was only slightly augmented (Figure $21 \mathrm{G}$ ). Genetic manipulation of cells typically result in a strong induction of immediate-early genes (Zhou et al., 2007). The deletion of TIP60 in confluent MEFs did not affect cell viability (Figure $21 \mathrm{H}$ ) nor did adenoviral transduction itself alter the circadian rhythm of a luciferase reporter (Figure 21I). Thus, abrogation of TIP60 function has major effects on locomotor activity and the rhythmic expression of clock genes in the SCN in vivo and in postmitotic MEFs highlighting the key role of TIP60 in the circadian clock and clock gene transcription.

\subsection{TIP60 Controls Productive Elongation of Circadian Transcripts}

The severe circadian phenotype in the mouse (Figure 20), the strong changes of clock gene expression in TIP60-deficient SCN and MEFs (Figure 20 and Figure 21), the TIP60mediated acetylation of BMAL1 at Lys538 (Figure 18), and the abrogated interaction between BMAL1 ${ }^{\mathrm{K} 538 \mathrm{R}}$ with BRD4 and P-TEFb (Figure 15), all suggest that deletion of TIP60 should prevent BRD4-P-TEFb recruitment and RNA Pol II pause release. Indeed, in confluent TIP60-deficient Dex synchronized fibroblasts, BMAL1 was hypoacetylated (Figure 21 A and Figure 22A) and there was a major loss of interaction between BMAL1 and BRD4 (Figure 22A and B) as well as between BMAL1 and CDK9 (Figure 22C). ChIP experiments showed that enrichment of BRD4 and CDK9 at the TSS of Dbp, Perl, and Rev-Erba genes was strongly reduced and Ser2-phosphorylated RNA Pol II occupancy was diminished (Figure 22D). Of note, deletion of Tip60 did not affect TFIIE $\alpha$ recruitment to the TSS of Dbp, Perl, and Rev-Erb $\alpha$ genes (Figure 22E). This suggests that TIP60, like acetylation of BMAL1 (Figure 17), plays an important role in transcription elongation rather than initiation. 
A

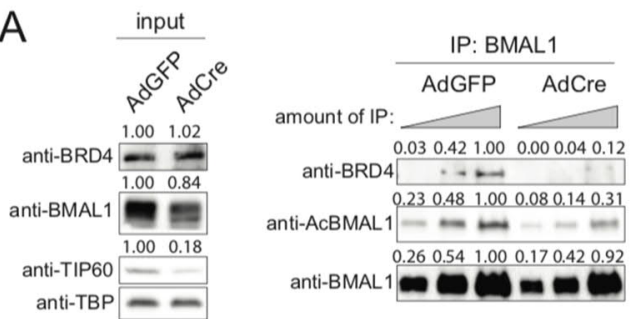

B

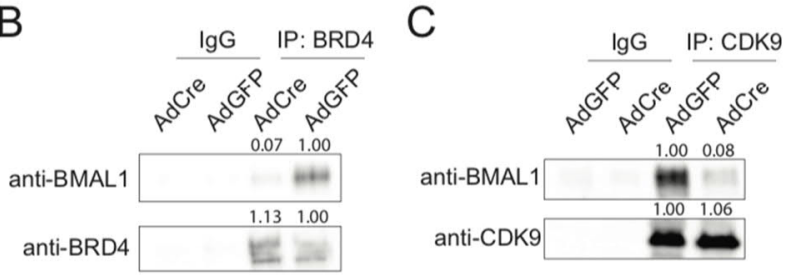

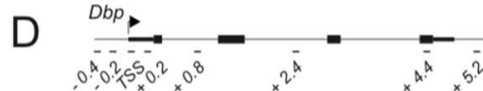
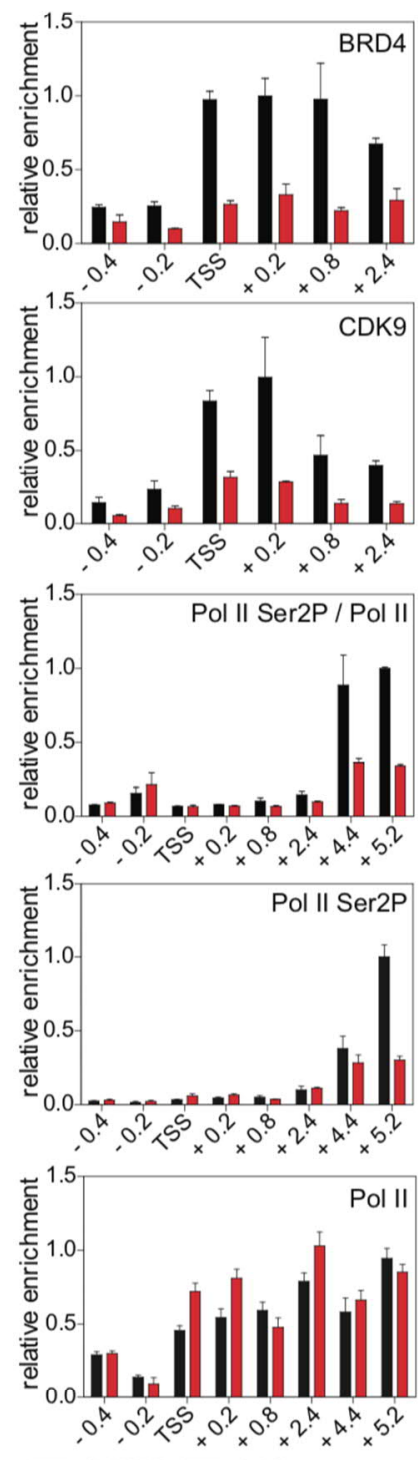

$E$

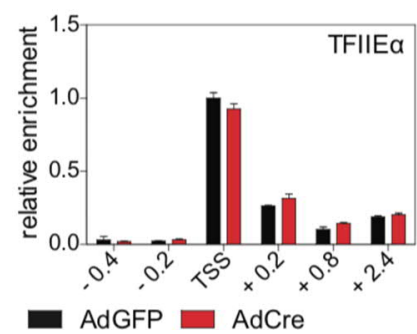

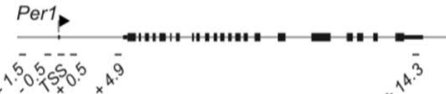
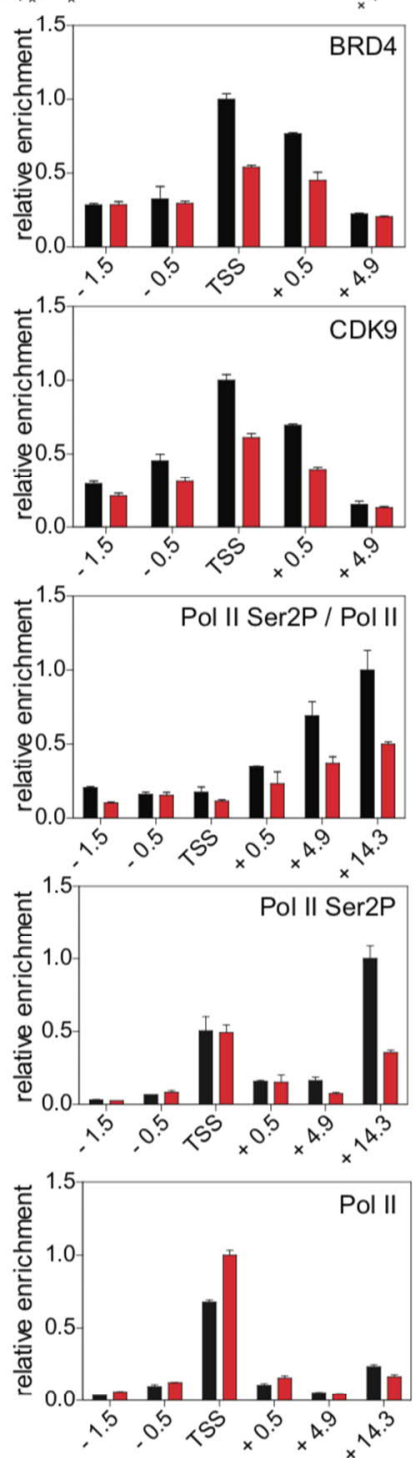

Rev-Erba
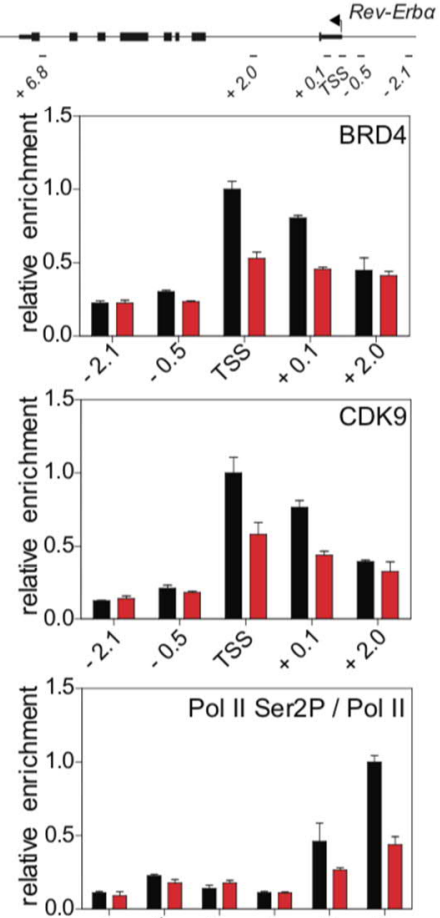

$2.0^{6} \times 5^{5} \times 0^{0} \times 2^{0} \times 6^{8}$
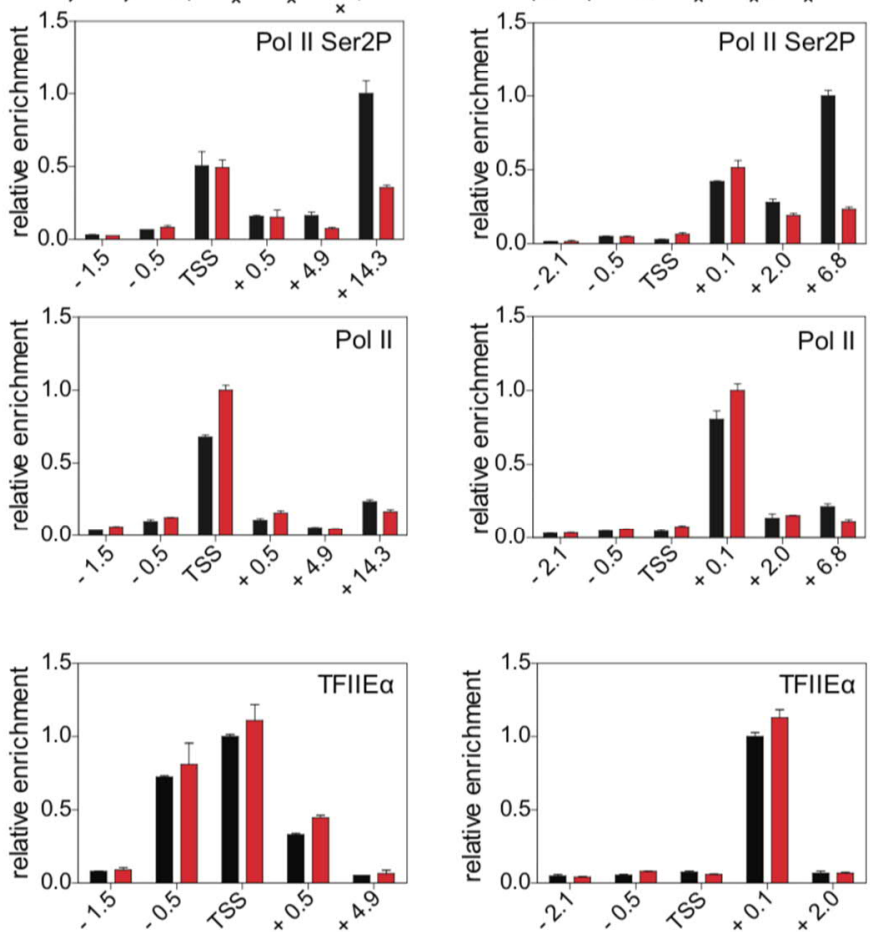

Figure 22: TIP60 Controls Productive Elongation.

(A) Interaction of BMAL1 with BRD4 in Tip60 ${ }^{f /-}$ MEFs transduced with either AdGFP (control) or AdCre (mutant). Immunoblots show results with increasing equivalents of 
IPs (two-fold dilutions) from nuclear extracts isolated from cells 24 hours after Dex synchronization. (B and C) BRD4 (B) or CDK9 (C) IPs from AdGFP or AdCre transduced Tip60 fl/- MEF nuclear extracts were immunoblotted with antibodies indicated. Analyses were carried out 24 hours after Dex synchronization. (D) ChIP analysis for BRD4, CDK9 and Ser2P-RNA Pol II (normalized to total RNA Pol II) in the promoter or the 3'-end of Dbp, Perl, and Rev-Erba genes for the cells shown in (A). (E) TFIIE $\alpha$ ChIP analysis of Dex-synchronized Tip60 $0^{f /-}$ MEFs transduced with either AdGFP or AdCre. All data are shown as mean \pm SEM $(n=3)$. Numerical values represent intensities of chemiluminescence signals of individual bands, normalized to wildtype and loading control for input samples.

\subsection{CRYs Oppose TIP60 and Repress Productive Elongation of Circadian Transcripts}

Collectively, the data presented so far provide evidence that TIP60-mediated acetylation of BMAL1 is an essential element of the positive limb of the circadian clock oscillator. TIP60-mediated acetylation of BMAL1 leads to the recruitment of BRD4 and of the pause release factor P-TEFb to clock gene TSSs. This, in turn, allows productive elongation of CLOCK-BMAL1-controlled genes. This positive limb of the circadian cycle is counteracted by a negative feedback mediated through PER and CRY proteins. This raises the question of whether and how these repressors could interfere with BRD4-P-TEFb recruitment and RNA Pol II pause release. One possibility is that nuclear accumulation of TIP60 is time-dependent, with a nadir at the end of the positive limb. Western blot analysis of nuclear lysates showed that this was not the case (Figure 24C). Alternatively, TIP60 could be displaced from the promoters by the negative regulators PERs and CRYs. CRY1 and CRY2 dynamically interact with the C-terminal transactivation domain of BMAL1 (Kiyohara et al., 2006; Sato et al., 2006; Xu et al., 2015) that comprises Lys538 that is acetylated by TIP60 and interacts with BRD4. To test this hypothesis, HEK293 cells were co-transfected with Bmall-myc, Tip60-V5, and with increasing amounts of either Cry1-flag or Cry2-V5. Both CRY1 and CRY2 out-competed TIP60 for binding to BMAL1 (Figure 23A and B). Furthermore, both CRY proteins markedly reduced acetylation of BMAL1 (Figure 23A and B). Binding competition and substrate acetylation were dose-dependent. PER2, on the other hand, did not affect the binding of TIP60 to BMAL1 and the acetylation status of the transcription factor (Figure 23C). CLOCKmediated acetylation of BMAL1 was also reported to facilitate recruitment of CRY1 to 
A

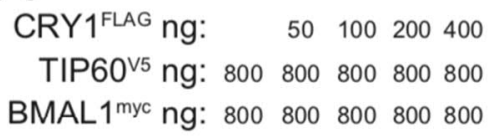

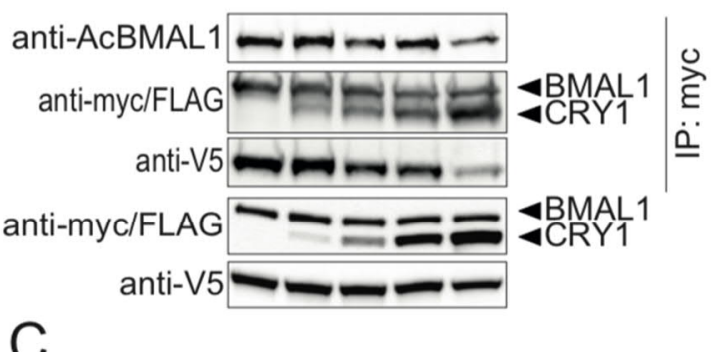

C

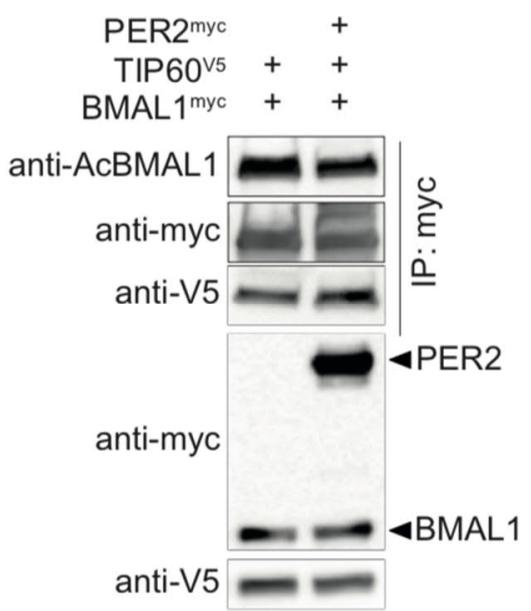

D

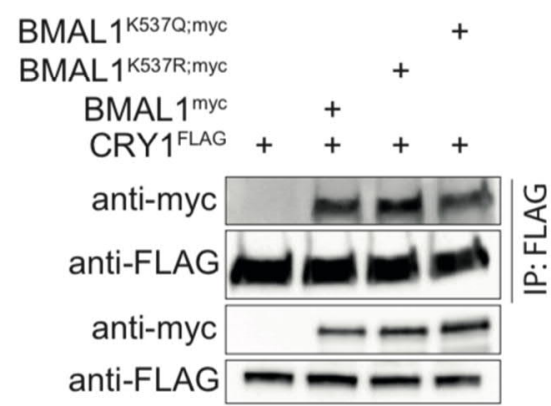

E

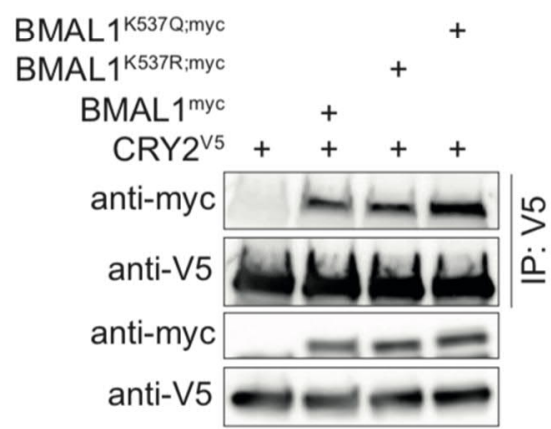

B

CRY2 ${ }^{\mathrm{V} 5} \mathrm{ng}: \quad 50 \quad 100200400$

TIP60 ${ }^{\mathrm{V}} \mathrm{ng}: 800800800800800$ BMAL1 ${ }^{\text {myc }}$ ng: 800800800800800

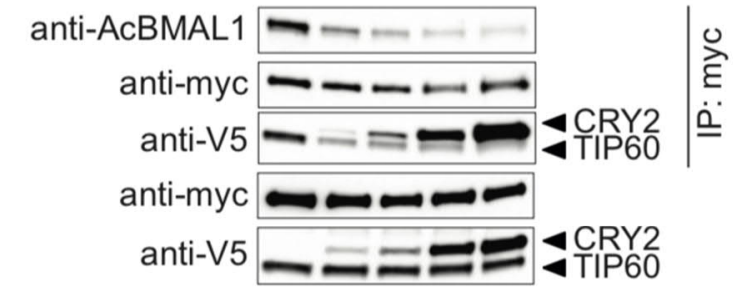

$\mathrm{F}$
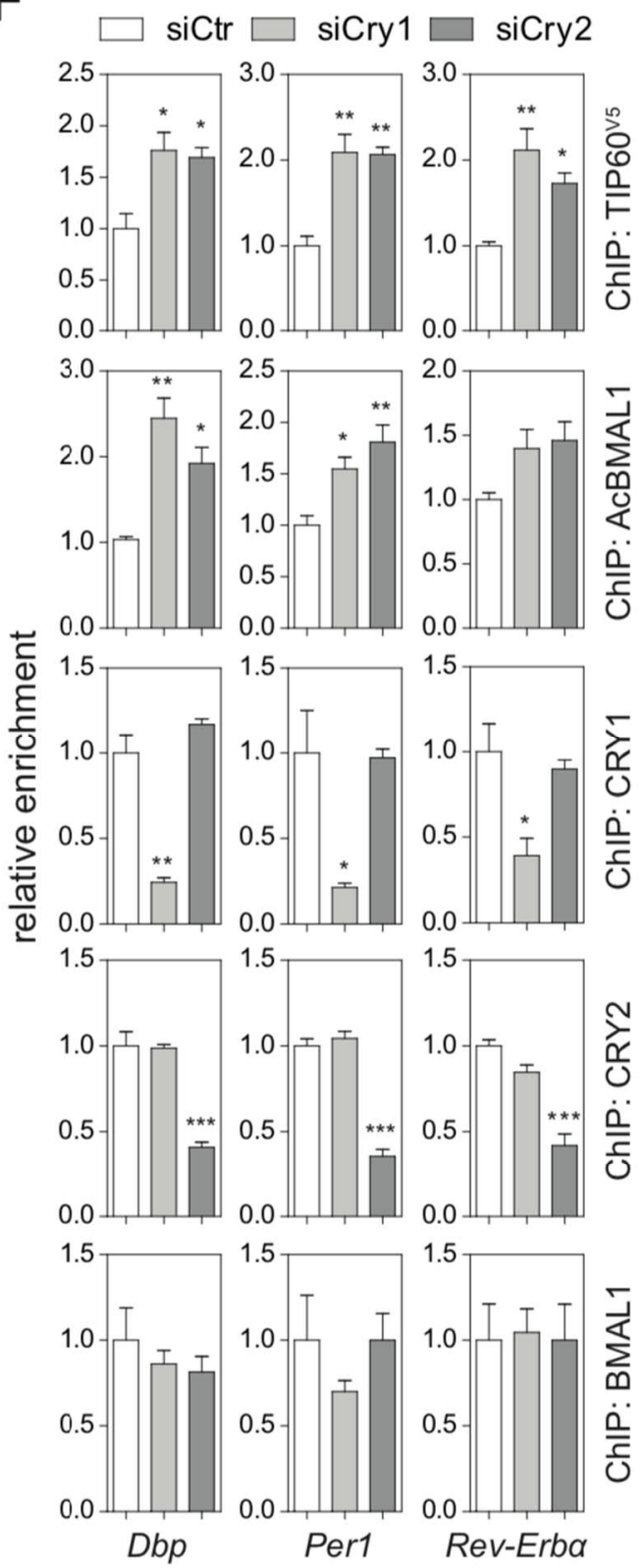

Figure 23: CRYs Compete with TIP60 for BMAL1 Binding.

(A - C) BMAL1 ${ }^{\text {myc }}$ IPs from transiently transfected HEK293T cells overexpressing $\mathrm{BMAL1}^{\mathrm{myc}}$ and TIP60 ${ }^{\mathrm{V} 5}$ together with increasing amounts of CRY1 ${ }^{\mathrm{FLAG}}$ (A) and CRY2 ${ }^{\mathrm{V} 5}$ (B) or steady amount of PER2 ${ }^{\text {myc }}(\mathrm{C})$. IPs were analyzed for the acetylation state of Lys538 of BMAL1 and binding of TIP60 ${ }^{\mathrm{V} 5}$ in response to CRY $1^{\text {flag }}, \mathrm{CRY} 2^{\mathrm{V} 5}$, or PER2 ${ }^{\mathrm{myc}}$. 
(D and E) Interaction of CRY1 ${ }^{\text {FLAG }}(\mathrm{E})$ or CRY2 ${ }^{\mathrm{V} 5}(\mathrm{~F})$ with BMAL1 $^{\text {myc }}$, BMAL1 $^{\mathrm{K} 538 \mathrm{R} ; \mathrm{myc}}$, and BMAL1 ${ }^{\mathrm{K} 538 \mathrm{Q} ; m y c}$ in transiently transfected HEK293T cells. Lysates were subjected to IPs and immunoblotted with antibodies indicated. (F) ChIP analysis for control, Cryl, and Cry 2 siRNA-depleted Tip60 ${ }^{-/}$; Tip60 ${ }^{V 5}$ MEFs at the promoters of Dbp, Perl, and $R e v-E r b \alpha$ genes for the proteins indicated. Data are shown as mean \pm SEM ( $=3 ; * \mathrm{p}<$ $0.05, * * \mathrm{p}<0.01$, and $* * * \mathrm{p}<0.001$, one-way ANOVA with Bonferroni post-test, relative to $\mathrm{siCtr})$.

the CLOCK-BMAL1 heterodimer, thereby promoting transcriptional repression (Hirayama et al., 2007). However, no difference in the interaction between CRY proteins with wildtype, non-acetylable (K538R), and acetyl-mimicking (K538Q) version of BMAL1 was detected (Figure 23D and E). Thus, CRY proteins seem to play a unique role in sequestering BMAL1 from the acetyltransferase TIP60, thereby regulating the acetylation status of BMAL1. To test whether this competition occurred directly on chromatin, CRY proteins were depleted by RNAi in MEFs in which endogenous TIP60 had been replaced by TIP60 ${ }^{\mathrm{V} 5}$. CRY depletion led to an increased occupancy of TIP60 55 and of acetylated BMAL1 at the promoters of Dbp, Perl, and Rev-Erba genes (Figure 23F). In CRY-deficient fibroblasts (CryDKO) the interaction between TIP60 and BMAL1 was markedly enhanced and acetylation of BMAL1 was strikingly upregulated (Figure 24A and C). Moreover, the interaction of BMAL1 with BRD4 was strongly augmented in the CryDKO fibroblasts (Figure 24A). CRY-mediated control of BMAL1 acetylation was not limited to fibroblasts but also occurred in the liver. In CRY-deficient liver samples, BMAL1 acetylation was strongly upregulated (Figure 25A). ChIP analysis revealed that acetylated BMAL1, BRD4, and CDK9 were highly abundant at the promoters of Dbp, Perl, and Rev-Erbo genes in CryDKO fibroblasts (Figure 24B) and Ser2-phosphorylated RNA Pol II was markedly increased (Figure 24B). A strong enrichment of acetylated BMAL1 at the promoters of Dbp, Perl, and Rev-Erba genes was also observed in CRY-deficient liver samples (Figure 25B). Consistent with previous work, Dbp, Perl, and Rev-Erb $\alpha$ mRNA levels were upregulated in CryDKO fibroblasts (Figure 24B) and CRY-deficient liver samples (Figure 25B) (Liu et al., 2007; Oishi et al., 2003). Taken together, these data support a general mechanism by which CRY proteins exert their inhibitory effect by preventing TIP60 from acetylating BMAL1, thereby repressing BRD4-P-TEFb recruitment and productive elongation. 
A

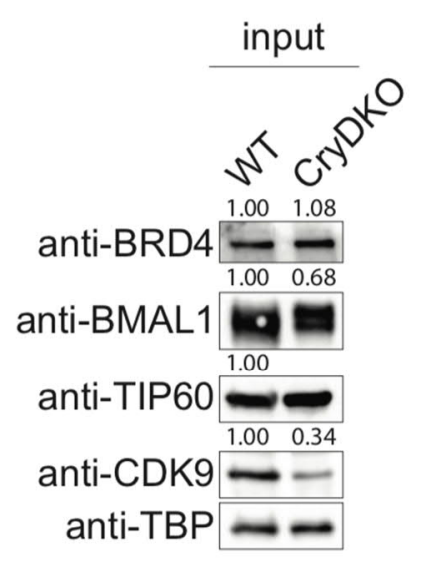

IP: BMAL1

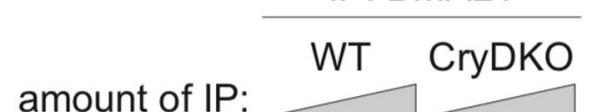

$1 . 0 0 \longdiv { 0 . 9 1 2 . 1 7 4 . 5 3 }$

\begin{tabular}{|c|c|c|}
\hline & 1.00 & 0.912 .174 .53 \\
\hline anti-BRD4 & & $\cdots-\infty$ \\
\hline $\begin{array}{l}1-11 \\
\end{array}$ & 0.411 .00 & $\begin{array}{r}1.28 .344 .86 \\
-7\end{array}$ \\
\hline & 0.180 .441 .00 & 1.111 .983 .72 \\
\hline & 0.210 .481 .00 & 0.621 .352 .76 \\
\hline
\end{tabular}

anti-AcBMAL1

$0.24 \quad 0.461 .00 \quad 0.22 \quad 0.521 .09$

anti-BMAL1 6 औ日

C

WT $\quad$ CryDKO

Time after Dex (hr) $24 \quad 30 \quad 36 \quad 42 \quad 24 \quad 30 \quad 36 \quad 42$

anti-AcBMAL1 $\because . \quad 10 \square$

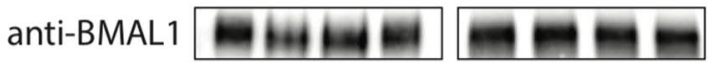

anti-TIP60

anti-BMAL1

anti-CRY1 $= \pm$

anti-CRY2 - - -

anti-TIP60 $--\longrightarrow-$

anti-TBP $=--\longrightarrow$

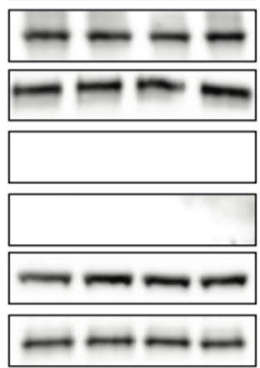

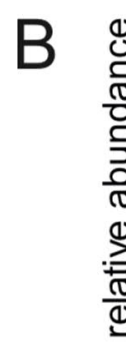
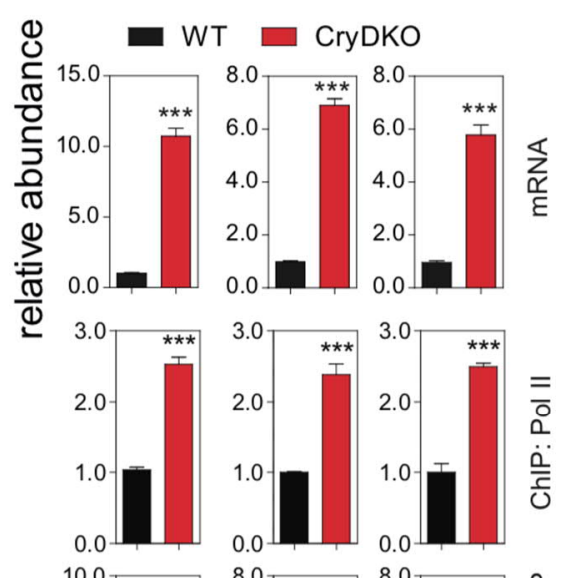

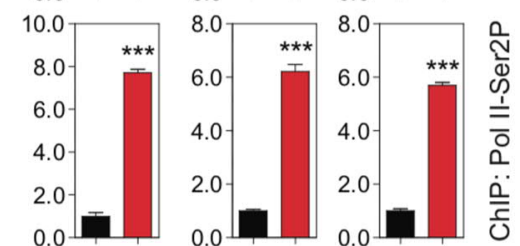
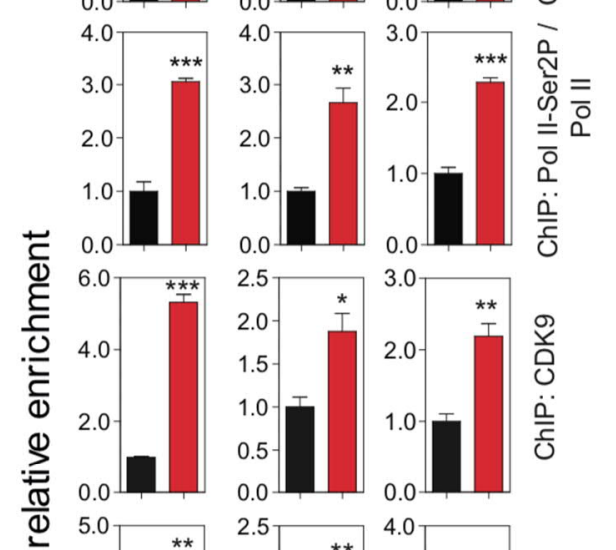

(1)
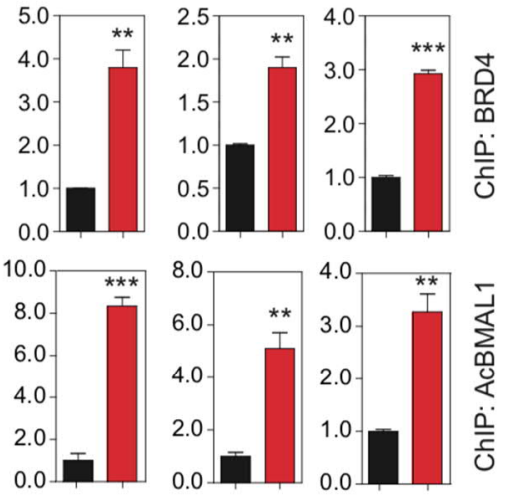

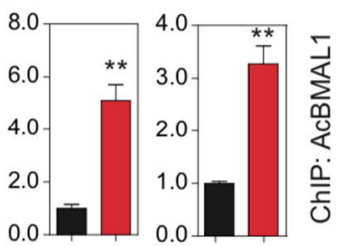
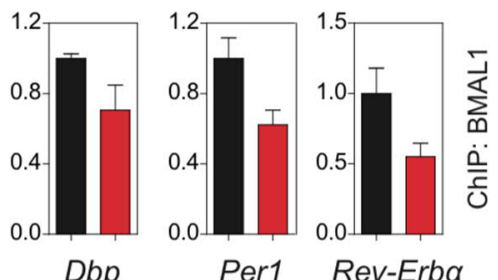

Figure 24: CRYs Control Transcription Elongation.

(A) Interaction of BMAL1 with BRD4 and TIP60 in control or CRY-deficient (CryDKO) fibroblasts. Immunoblots show results with increasing equivalents of IPs (two-fold dilutions) from nuclear extracts harvested from unsynchronized cells. Numerical values represent intensities of chemiluminescence signals of individual bands, normalized to wildtype and loading control for input samples. (B) ChIP (bottom) and mRNA (top) analysis of unsynchronized wildtype and CryDKO fibroblasts. ChIP shows enrichment 
of BMAL1, acetylated BMAL1, BRD4, CDK9, and Ser2P-RNA Pol II (normalized to total RNA Pol II) at the promoter or 3'-end (Ser2P-RNA Pol II and RNA Pol II) of Dbp, Perl, and Rev-Erba genes. Data are shown as mean \pm SEM $(\mathrm{n}=3 ; * * \mathrm{p}<0.01$, and ***p $<0.001$, Student's t-test). (C) Immunoblot analysis of wildtype and CryDKO fibroblasts nuclear extracts and BMAL1 IPs over a 24-hour time course with antibodies indicated.

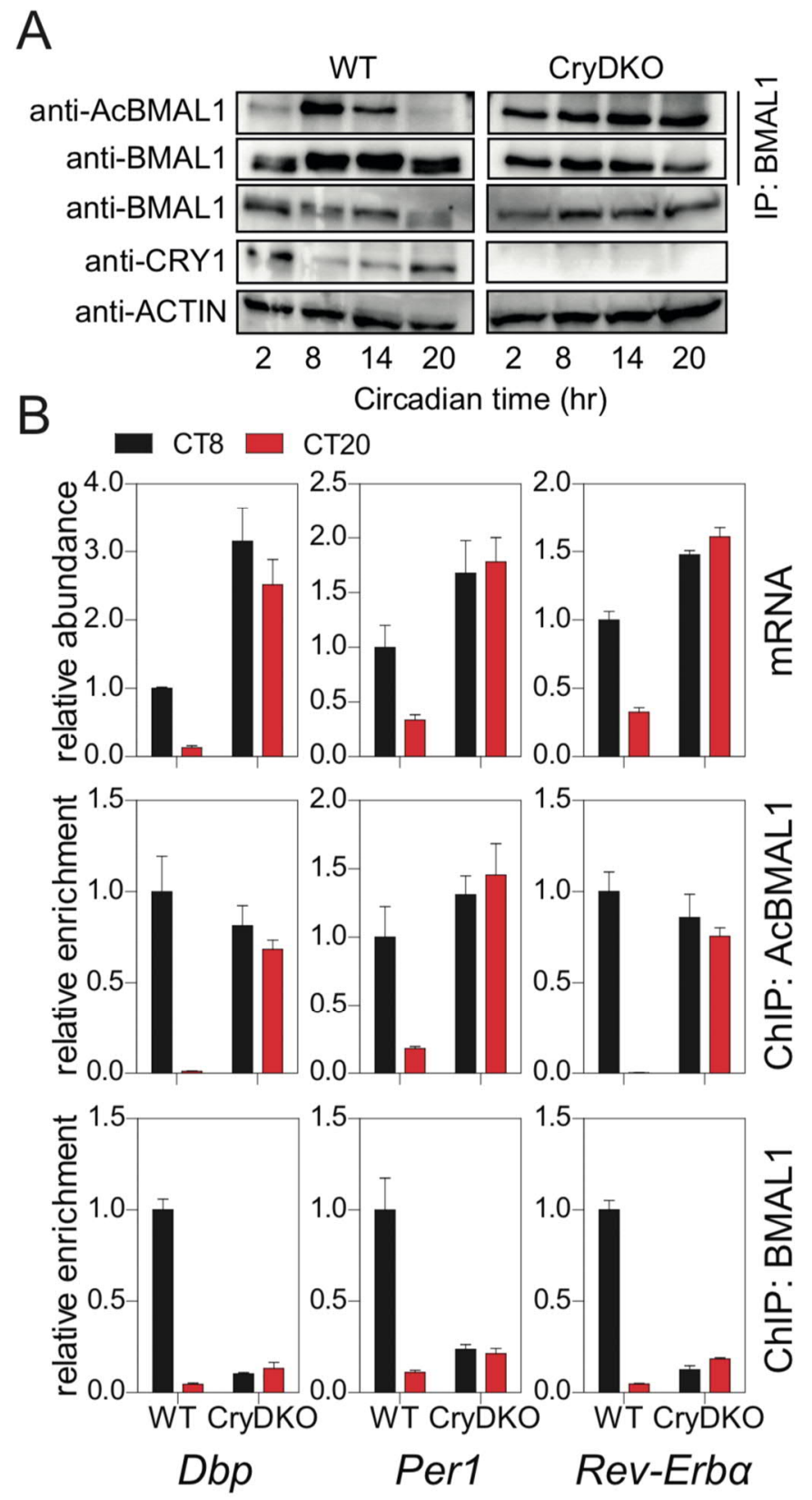

Figure 25: CRYs Control Lys538 Acetylation of BMAL1 in the Liver.

(A) Immunoblot analysis of wildtype and CRY-deficient liver nuclear extracts and BMAL1 IPs over a 24-hour time course with antibodies indicated. (B) ChIP (bottom) and mRNA (top) analysis of wildtype and CRY-deficient liver samples carried out at CT8 and CT20. ChIP shows enrichment of BMAL1 and acetylated BMAL1 at the promoter of Dbp, Perl, and Rev-Erb $\alpha$ genes. Data are shown as mean $\pm \operatorname{SEM}(\mathrm{n}=3)$. 


\subsection{Rhythmicity of Productive Elongation of Circadian Transcripts}

TIP60 can access BMAL1 at the promoters during the positive limb of the clock cycle, but when CRYs are abundant during the negative limb, this access is hindered. This interplay between positive and negative regulation, characteristic for the circadian cycle, should also be seen in temporal occupancy profiles of pause release indicators at the promoters of E-box-controlled clock genes. In wildtype fibroblasts, the peak of interaction between TIP60 and BMAL1 occurred approximately 24 hours after synchronization of the cells (Figure 24C) (activation phase). During this phase, the strongest enrichment of BMAL1 and of acetylated BMAL1 at the promoters of $D b p$, Perl, and Rev-Erba genes was observed (Figure 26A, green). The concentration of acetylated BMAL1 in nuclear extracts also showed its maximum by that time (Figure 26B). The occupancy of BRD4 also peaked at target gene promoters (Figure 26A) and Ser2 phosphorylation of RNA Pol II showed a peak (Figure 26A) at that time. Consequently, the abundance of Dbp, Perl, and Rev-Erb $\alpha$ mRNA peaked between 24$28 \mathrm{~h}$ (Figure 26A). A similar situation was also be observed in the wildtype liver. The peak of acetylated BMAL1 occurred at circadian time (CT) 8 (Figure 25A), a time period that falls into the activation phase (Koike et al., 2012). During that time, the strongest enrichment of BMAL1 and of acetylated BMAL1 at the promoters of $\mathrm{Dbp}, \mathrm{Perl}$, and Rev-Erb $\alpha$ genes was observed (Figure 27A) and expression of Dbp, Perl, and Rev-Erba showed its maximum (Figure 27A). Of note, CRY proteins peak later in the circadian day and thus cannot substantially interfere with BRD4 recruitment, RNA Pol II pause release and productive elongation in the fibroblasts (Figure 26A). The gradual rise of nuclear CRY proteins (Figure 26B) and of Dbp promoter associated CRY1 (Figure 26C) seen during the repression phase was accompanied by a reduction of the interaction of TIP60 with BMAL1 (Figure 24C), a decline of chromatin-bound acetylated BMAL1, a reduction of BRD4 recruitment to circadian promoters, a drop of Ser2-phosphorylated RNA Pol II and a dampening of E-box-controlled gene expression (Figure 26A, red). Such a CRY dependent reduction of BMAL1 acetylation was also observed in wildtype liver. The rise of nuclear (Figure 25A) and chromatin associated (Figure 27B) CRY1 protein seen during the repression phase was accompanied by a reduction of chromatin-bound acetylated BMAL1 (Figure 27A) and dampening of Dbp, Per1, and Rev-Erb $\alpha$ expression (Figure 27A). Thus, control of BMAL1 acetylation by CRY proteins appears to be a general mechanism of CRY-mediated repression that is not limited to fibroblasts. Taken together, the data show that TIP60-dependent BRD4 recruitment, RNA Pol II pause release, 
productive elongation, and the opposing effects of chromatin associated CRY proteins on transcription are precisely timed over the circadian cycle and in this way exert a temporal control over the circadian clock oscillator.
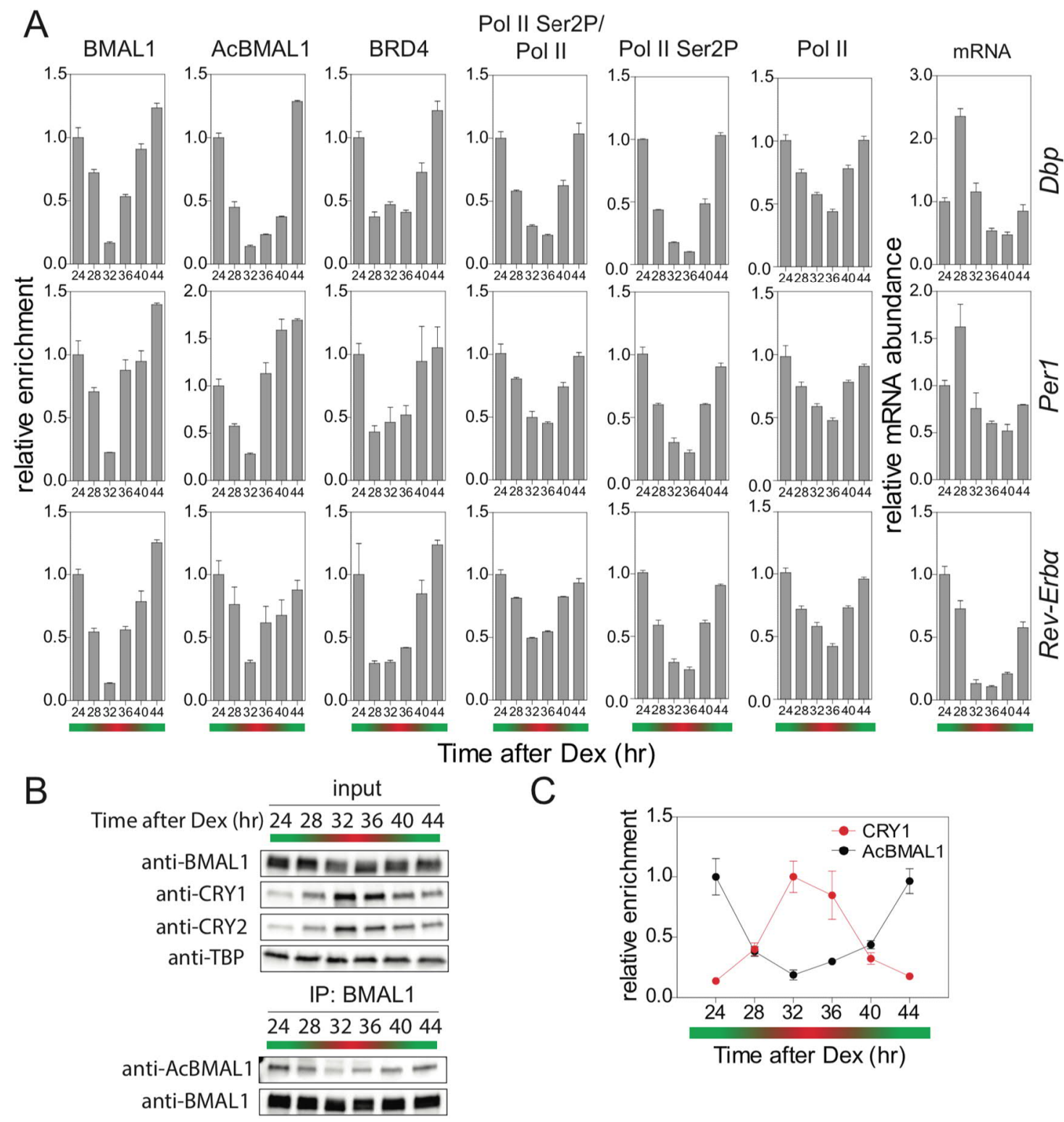

Time after Dex (hr)

Figure 26: Rhythmic Profile of Productive Elongation.

(A) ChIP profiles of Dex-synchronized MEFs over a course of 24 hours. ChIP analysis show time-of-day-dependent enrichment of BMAL1, acetylated BMAL1, BRD4, and Ser2P-RNA Pol II (normalized to total RNA Pol II) at the promoter or 3'-end (Ser2PRNA Pol II and RNA Pol II) of Dbp, Perl, and Rev-Erb $\alpha$ genes. The rightmost column reveals time-dependence of mRNA accumulation for these genes. (B) Immunoblot analysis with the indicated antibodies of Dex-synchronized fibroblast nuclear extracts and BMAL1 IPs. (C) ChIP analysis of acetylated BMAL1 and CRY1 for the Dbp promoter in Dex-synchronized MEFs over a course of 24 hours. All data are shown as mean \pm SEM $(\mathrm{n}=3)$. The color bars represent the activation (green) and repression (red) phases of the circadian cycle. 

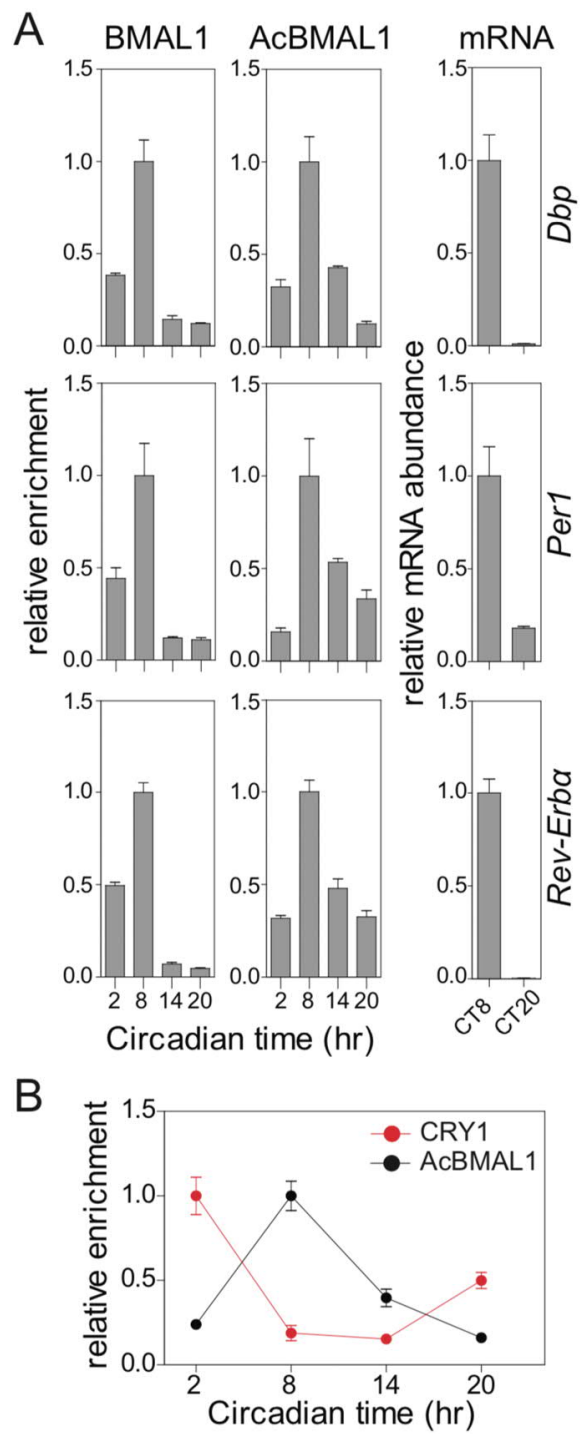

Figure 27: Rhythmic Profile of BMAL1 Acetylation in the Liver.

(A) Time-of-day-dependent enrichment of BMAL1 and acetylated BMAL1 at the promoter of Dbp, Perl, and Rev-Erba genes in the liver. The rightmost column reveals time-dependence of mRNA accumulation for these genes. (B) ChIP analysis of acetylated BMAL1 and CRY1 for the Dbp promoter in wildtype liver over a course of 24 hours. All data are shown as mean $\pm \operatorname{SEM}(n=3)$. 


\section{Chapter 4: Discussion}

Data from genome-wide ChIP studies (Koike et al., 2012; Menet et al., 2012; Rey et al., 2011) in particular, Koike et al. propose that at the onset of the circadian cycle, the repressor CRY1 is associated with the chromatin-bound CLOCK-BMAL1 located near RNA Pol II. In this complex RNA Pol II is bound to the clock gene promoters but is not competent to drive RNA synthesis. Upon degradation of CRY1, coactivators (e.g. p300) are recruited to the CLOCK-BMAL1 heterodimer complex, followed by transcription of circadian clock genes encoding CRY and PER repressor proteins. To ensure the precision of the circadian clock, transcription needs to be accurately timed and synchronous across a population of cells. Although promoter proximal pausing is a regular step of the transcription cycle, the release of this process can be a crucial regulatory step in transcriptional activation of certain gene classes. Thus, promoter proximal pausing and release into productive elongation offer a mechanism capable to accurately time transcription (Core and Adelman, 2019; Jonkers and Lis, 2015; Liu et al., 2015). Evidence for RNA Pol II pausing to occur at circadian promoters can be found in global run-on sequencing (GRO-Seq) analyses measuring transcriptionally engaged RNA Pol II during the circadian cycle (Fang et al, 2014). In this study, a sharp peak of RNA Pol II downstream of the TSS of the Rev-Erb $\alpha$ gene was detected during the repression state, raising the possibility that RNA Pol II is in a paused state at this particular promoter. Furthermore, genome-wide ChIP experiments (Koike et al., 2012) reveal a peak of RNA Pol II Ser5 phosphorylation at a time when the CLOCK-BMAL1-CRY1 complex is associated with circadian promoters near transcriptionally paused RNA Pol II. Such RNA Pol II Ser5 phosphorylation is a main indicator for promoter-proximal pausing (Harlen and Churchman, 2017). In addition, genome-wide and time-resolved quantitative analysis of RNA Pol II abundance around TSS regions and gene bodies show a peak of RNA Pol II binding near the TSS of clock genes at the same time when the CLOCK-BMAL1CRY1 complex orchestrates circadian promoters (Zhu et al., 2018). Thus, the RNA Pol II peaks at the TSS of clock genes, at the onset of the circadian cycle, likely represent paused RNA Pol II. A recent study using data from various published sources and mathematical modeling also suggests that, at least for strong circadian promoters, RNA Pol II release could be a critical regulatory step of circadian transcription (Westermark, 2016). The present work now provides both, experimental evidence and mechanistic 
insights into circadian regulation of RNA Pol II pause release and suggests that this process could be an important regulatory step of the mammalian circadian clock.

This study proposes that TIP60 acetylates BMAL1 on-chromatin which then triggers the recruitment of the co-activator BRD4 to the acetylated transcription factor BMAL1 and hence to clock gene promoters. BRD4 recruits the pause release factor P-TEFb, whose kinase subunit CDK9 phosphorylates Ser2 of RNA Pol II leading to a release of RNA Pol II from the paused state enabling productive elongation of $E$-box-containing clock genes (Figure 28). For this sequence of events multiple lines of evidence are provided. (1) In TIP60-deficient cells, Lys538 acetylation of BMAL1 was strongly diminished leading to abolishment of BRD4-P-TEFb recruitment to Dbp, Perl, and Rev-Erba genes and prevention of RNA Pol II pause release. (2) In BMAL1 ${ }^{\mathrm{K} 538 \mathrm{R}}$ cells, in which Lys538 was replaced by an arginine, even though TIP60 was still recruited to the mutated protein, the interaction between BMAL1 and BRD4-P-TEFb was abrogated, preventing RNA Pol II pause release and productive elongation. (3) Pharmacological inhibition of BRD4 abolished BRD4-P-TEFb recruitment to circadian promoters and consequently impaired RNA Pol II pause release of E-box-containing clock-controlled genes. (4) The dysregulation of the circadian rhythm of locomotor activity in the mouse and perturbation of circadian gene expression in the SCN and in MEFs provide strong genetic evidence

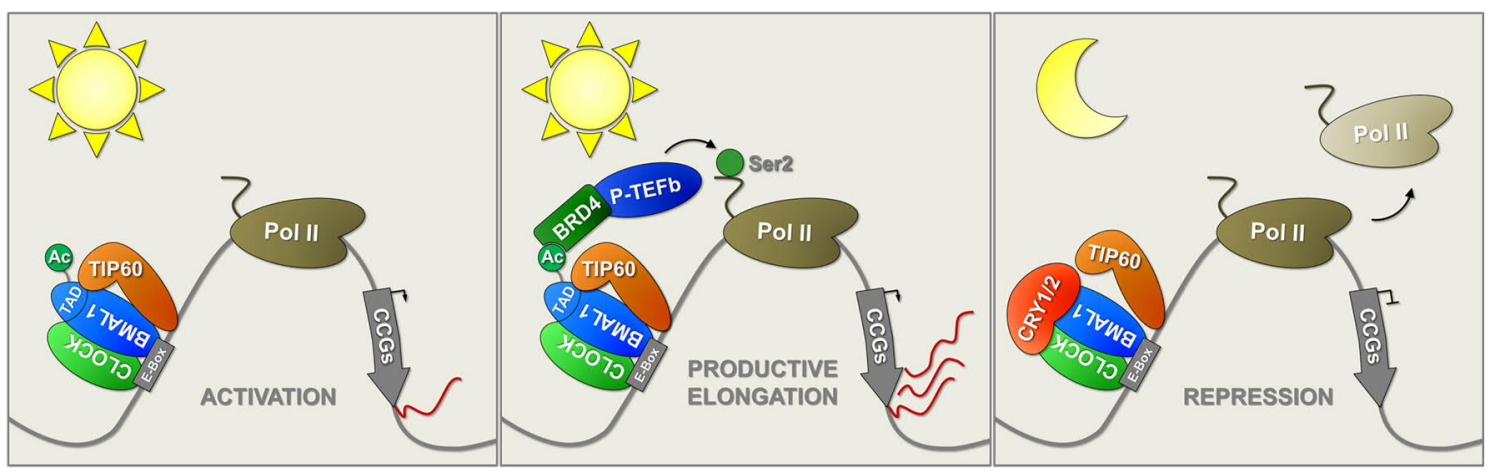

Figure 28: Model of RNA Pol II Pause Release Control at E-box-containing Clock Genes.

This study provides evidence that the positive and negative phases of the circadian oscillator are linked through TIP60. The circadian clock cycle is initiated by CLOCKBMAL1 binding to E-boxes of clock genes followed by a TIP60-mediated acetylation of chromatin bound BMAL1, which then triggers the recruitment of BRD4. BRD4 recruits P-TEFb, whose kinase subunit CDK9 phosphorylates Ser2 of RNA Pol II leading to a release of RNA Pol II from the paused state enabling productive elongation. During the repression phase, when CRY proteins are highly abundant at clock gene promoters, transcription elongation ceases, most likely by sequestering BMAL1 from TIP60 and prevention of TIP60-driven acetylation of BMAL1. 
for a key role of TIP60 in the circadian clock. Taken together, these lines of evidence suggest that rhythmic control of RNA Pol II pause release may be a main process involving TIP60. Furthermore, the study illustrates that RNA Pol II pause release is an additional regulatory step that contributes to the temporal control of the mammalian circadian clock.

The transcription cycle consists of several sequential steps that are regulated in response to cellular signals or cues (Core and Adelman, 2019). First of all, TSSs must be made accessible to the transcription machinery, which often requires cooperation between transcription factors and chromatin modifiers and remodelers. The evidence that this also applies to CLOCK-BMAL1 comes from a genome-wide ChIP study showing that the histone acetyltransferase p300/CBP has many common binding sites with the CLOCKBMAL1 heterodimer (Koike et al., 2012). Furthermore, CLOCK-BMAL1 was reported to interact with BRG1 (Brahma-related gene 1), the catalytic subunit of the mammalian SWI/SNF (SWItch/Sucrose Non-Fermentable) chromatin-remodeling complex (Kim et al., 2014). Although first studies using a computational approach in combination with genome-wide RNA Pol II ChIP data concluded that RNA Pol II pausing plays a negligible role in circadian transcription regulation (Le Martelot et al., 2012), accumulation of RNA Pol II observed at TSSs of clock-controlled genes clearly shows that the release from promoter proximal pausing is a rate limiting step in the transcription cycle of these genes. This is also supported by a recent computational study using a multitude of circadian genome-wide datasets showing that transcription initiation in combination with RNA Pol II pause release are essential for strong rhythmic clock gene expression (Westermark, 2016). Thus, CLOCK-BMAL1 plays a multifaceted role during transcription activation. It does not only regulate transcription initiation (Koike et al., 2012; Le Martelot et al., 2012) but also regulates RNA Pol II pause release after TIP60-mediated acetylation, as illustrated in this study. One advantage of the separate regulation of transcription initiation and RNA Pol II pause release is that signaling pathways can influence one or both steps, enabling the integration of conflicting or magnifying signals (Figure 29) (Core and Adelman, 2019). The result is a dynamic and precise control of gene expression that can be found in a variety of biological processes such as immune responses, hormone signaling, or early development (Core and Adelman, 2019). Binding of CLOCK-BMAL1 to the promoters of clock-controlled genes is therefore not sufficient to induce strong rhythmic gene expression and requires an addition signal in the form of the acetyltransferase TIP60. This could also explain why CLOCK-BMAL1 binding to the 
DNA not always results in rhythmic gene expression and shows divergent transcriptional output (Koike et al., 2012; Menet et al., 2012; Rey et al., 2011) and why nuclear amount of BMAL1 is lowest during the activation phase (Lee et al., 2001). Thus, not the mere presence of BMAL1 is essential for the transcriptional output of the transcription factor, but its correct posttranslational modification. This bimodal regulation of circadian transcription regulation could also allow the integration of environmental cues to entrain the system to the new zeitgeber such as light, temperature, and food. Thus, CLOCKBMAL1 not only appears to be involved in the initial stages of RNA Pol II recruitment and initiation (Koike et al., 2012; Le Martelot et al., 2012) but also controls RNA Pol II pause release to determine the ultimate transcriptional output of the clock-controlled genes.
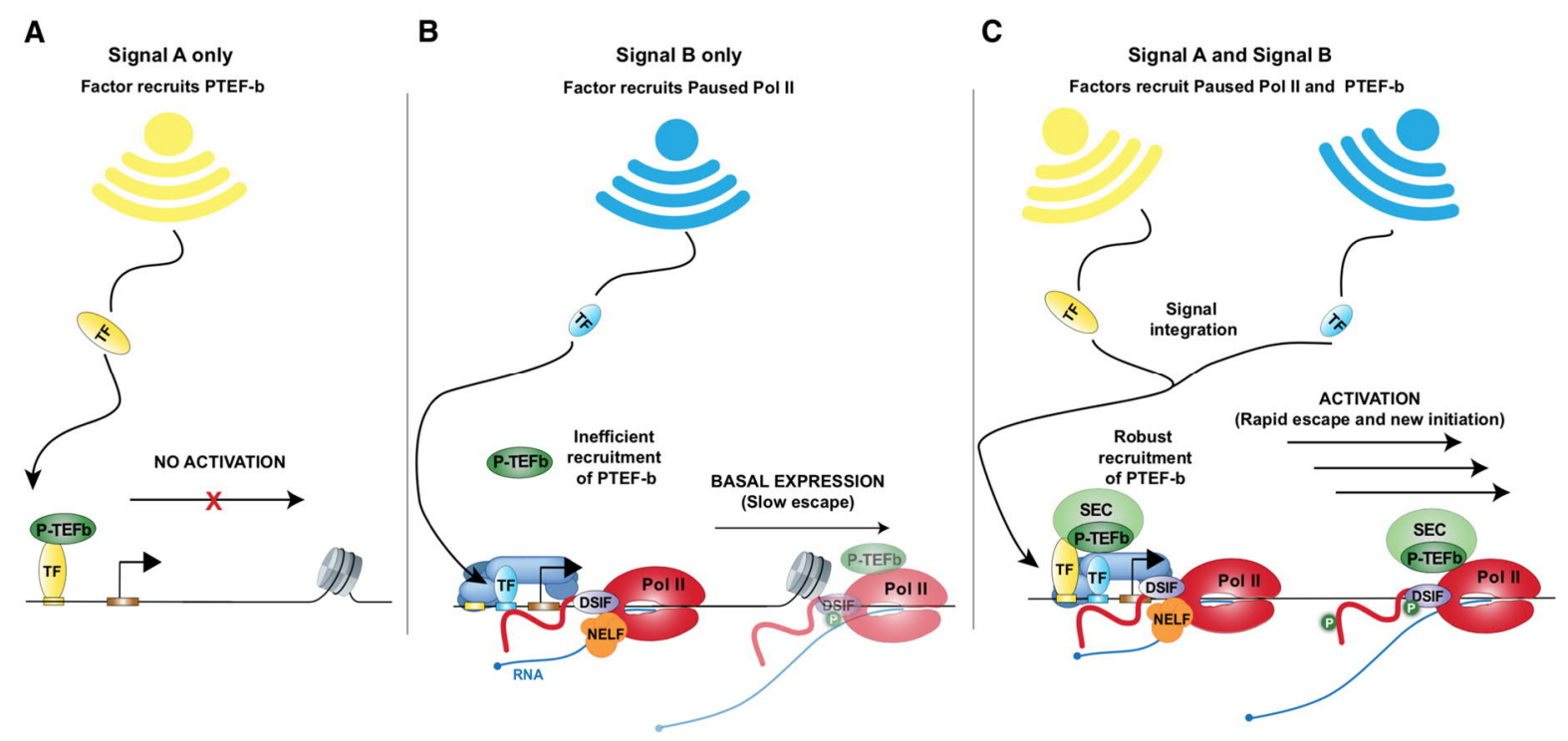

Figure 29: Transcription factors can control initiation and/or RNA Pol II pause release.

(A) Recruitment of P-TEFb by a TF is not sufficient to activate gene transcription. (B) TF-mediated stimulation of transcription initiation will result in low level of gene expression. (C) Stimulation of both initiation and RNA Pol II pause release are essential for strong and rapid gene activation. Figure adapted from Core and Adelman (2019).

TIP60 is evolutionarily highly conserved and is involved in the regulation of a series of cellular processes (see introduction). Thereby, TIP60 has mainly the role of an enzymatically active cofactor in larger multiprotein complexes (Jacquet et al., 2016; Judes et al., 2015). This study suggests a similar function of TIP60 within the circadian clock. Although TIP60 deficiency leads to a severe circadian phenotype, the mechanism that is proposed would classify TIP60 as a coactivator/cofactor and not as a core clock 
protein. Furthermore, the study leaves open the question of whether TIP60 is recruited alone or in a larger complex to CLOCK-BMAL1. TIP60 is an essential protein whose deletion results in early embryonic lethality at the blastocyst stage (Hu et al., 2009). In contrast, TIP60 acetyltransferase mutant mice, encoding amino acid substitutions in the active site, exhibit developmental defects later than TIP60 null mutant mice. This emphasizes that not all functions of the TIP60 complex rely on its acetyltransferase activity but the acetyltransferase-independent regulation of gene expression also plays an important role (Acharya et al., 2017). In addition to the acetyltransferase TIP60, the TIP60 complex harbors another subunit with chromatin remodeling activity. The p400 protein catalyzes ATP-dependent incorporation of histone variant H2A.Z or H3.3 into chromatin (Gévry et al., 2007; Mizuguchi et al., 2004; Pradhan et al., 2016). Both histone variants are often enriched around TSSs promoting recruitment of RNA Pol II and gene activation (Melters et al., 2015). CLOCK-BMAL1 binding at clock-controlled gene promoters strongly correlates with rhythmic changes in H2A.Z occupancy and BMAL1-deficency results in a severe loss of H2A.Z deposition at these sites (Menet et al., 2014). Thus, recruitment of TIP60 complex to E-box bound CLOCK-BMAL1 could lead to two distinct events, the acetylation of BMAL1 by TIP60 and the ATP-dependent incorporation of H2A.Z at the TSSs by p400. Consequently, the TIP60 complex could control both RNA Pol II recruitment to the TSSs of clock-controlled genes and the RNA Pol II pause release and productive elongation of these genes.

Although it has been proposed that acetylation of BMAL1 at Lys538 by CLOCK peaks within the repression phase and enables recruitment of CRY proteins (Hirayama et al., 2007) the data presented here do not support this claim. This study shows that TIP60mediated acetylation of BMAL1 occurs during the activation phase of the clock cycle, a phase when CRY proteins are low abundant. This is in line with the latest data from the Sassone-Corsi laboratory (Koronowski et al., 2019; Nakahata et al., 2008; Welz et al., 2019). Co-immunoprecipitation experiments did also not show any differences in the interaction of BMAL1 or BMAL1 ${ }^{\mathrm{K} 538 \mathrm{R}}$ with $\mathrm{CRY}$ proteins, arguing against the proposed model by Hirayama and co-workers (Hirayama et al., 2007). A recent study using a mouse model that reconstitutes only the hepatic clock in an otherwise clock-deficient animal (Koronowski et al., 2019) showed that peak of BMAL1 acetylation is phase advanced by approximately 4 hours compare to wildtype liver. Interestingly, recruitment of BMAL1 to the promoters of Dbp and Perl and the expression of these genes was also phase advanced, underlining the importance of BMAL1 acetylation during the activation phase. 
The finding of strong acetylation of Lys538 of BMAL1 in CLOCK-deficient fibroblasts and the absence of an effect on the BMAL1 acetylation status in a reaction with a catalytically inactive CLOCK mutant protein raises also some doubt about the efficiency of CLOCK as an acetyltransferase for BMAL1. A slight increase in BMAL1 acetylation after transfection of wildtype or acetyltransferase deficient CLOCK (Figure 18A) supports the idea that the formation of a CLOCK-BMAL1 complex favors the recruitment of acetyltransferases such as TIP60. Thus, the enhanced histone acetyltransferase activity of CLOCK after co-transfection with BMAL1 (Doi et al., 2006) might be the result of augmented TIP60 recruitment. It is noteworthy that CLOCK acetylates argininosuccinate synthase in the cytosol of U2OS cells (Lin et al., 2017). Since the experiments conducted in this study are aimed at the transcriptional regulation of the clock, they were carried out with nuclear extracts and do not address the cytosolic acetyltransferase activity of CLOCK.

Cells express a multitude of acetyltransferases including TIP60 and CBP/p300. $\mathrm{CBP} / \mathrm{p} 300$ was reported to directly bind and mediate the transactivation activity of BMAL1 (Etchegaray et al., 2003; Koike et al., 2012; Takahata et al., 2000). However, in fibroblasts, but also in the SCN, deletion of TIP60 resulted in a nearly complete loss of rhythmic expression of numerous circadian genes suggesting that in these tissues and for these genes TIP60 function is non-redundant. Although it has been reported that p300 acetylates BMAL1, it does this at Lys500 and not at Lys538 (Weinert et al., 2018). It cannot be ruled out that p300 and TIP60 could act cooperatively and promote the acetylation of BMAL1 (Xiao et al., 2014). However, recombinant TIP60 was able to acetylate BMAL1 in vitro, arguing that BMAL1 is a direct substrate of TIP60. The abundance of BRD4 at clock gene promoters was drastically reduced in BMAL1 ${ }^{\mathrm{K} 538 \mathrm{R}}$ cells, but it is likely that there are other substrates that are acetylated including histones H3 and H4 present at promoters of circadian genes (DiTacchio et al., 2011; Etchegaray et al., 2003; Koike et al., 2012; Ripperger and Schibler, 2006; Vollmers et al., 2012), to which BRD4 could also be recruited and affect the circadian clock.

Previous studies showed that CRY proteins directly interact with CLOCK-BMAL1 and are part of a larger Pol II-containing complex. The contacts between BMAL1 and CRY proteins map to the C-terminal transactivation domain of BMAL1 (Kiyohara et al., 2006; Sato et al., 2006; Xu et al., 2015) that also comprises Lys538 that is acetylated by TIP60. Thus, CRY proteins have the opportunity to prevent TIP60-mediated acetylation of BMAL1. That this is indeed the case in vivo, is illustrated in experiments with CRY- 
deficient and Cry1- and Cry2-depleted fibroblasts. In these cells, interaction between TIP60 and BMAL1 was evidently not inhibited, resulting in a persistent Lys538 acetylation of BMAL1 throughout the circadian cycle. Such a loss of CRY function massively boosted BRD4-P-TEFb recruitment to BMAL1 followed by RNA Pol II pause release and the productive elongation of E-box-containing circadian genes. Conversely, if CRY proteins were overexpressed, interaction between TIP60 and BMAL1 was prevented, BMAL1 was hypoacetylated, BRD4-P-TEFb was not recruited, and productive elongation was impaired. It thus appears that the negative feedback loop of the circadian cycle involves, at least in part, a CRY-mediated repression of acetylation of BMAL1 by TIP60 resulting in an obstruction of BRD4-P-TEFb recruitment and RNA Pol II pause release. This study proposes that positive and negative signals converge on the same molecular target (Figure 28).

The outstanding role of CRY1, within the molecular clock, is illustrated by the fact that CRY1 plays a more indispensable role for rhythmic clock gene transcription than CRY2 and seems to have a stronger repressor capacity for CLOCK-BMAL1-mediated transcription than its homolog (Khan et al., 2012; Rosensweig and Green, 2018). At a behavioral level, CRY1 and CRY2 single knockout mice also show distinct differences in period length (van der Horst et al., 1999; Vitaterna et al., 1999). While CRY1-deficient mice illustrate shorter period, surprisingly, CRY2-deficient mice show longer period under constant condition. Based on the prosed model, absence of CRY1 during the late repression phase would allow TIP60 earlier to form a complex with CLOCK-BMAL1 and thereby reduce the time of promoter proximal pausing and accelerate the clock. Loss of promoter proximal pausing and impaired synchronized expression of CLOK-BMAL1 controlled genes is probably also the reason why individual cells like fibroblast, SCN neurons, or tissue explants from CRY1-deficient mice lack stable rhythms (Liu et al., 2007; Wong et al., 2018). By contrast, in CRY2-deficient mice (a CRY1-driven clock), CRY1 is more abundant in the nucleus and shows extended expression reaching into the early circadian day (Stratmann et al., 2010). Thus, stabilization of CRY1 and prolongation of promoter proximal pausing allows it to delay the onset of CLOCK-BMAL1-driven transcription, thereby extending the limb of the negative feedback. Nuclear stabilization of CRY-proteins either through pharmacological treatment with a small molecule named KL001 (Hirota et al., 2012) or genetic manipulations (Foteinou et al., 2018; Godinho et al., 2007; Patke et al., 2017; Siepka et al., 2007) were previously reported to slow down the circadian clock likely by extending the pause duration time of RNA Pol II at E-box- 
driven clock genes. Tissue explants from CRY2-deficient mice show circadian rhythms that are more robust und stable compared to wildtype counterparts (Liu et al., 2007; Wong et al., 2018). An extension of promoter proximal pausing could lead to increased synchronous expression of CLOCK-BMAL1-target genes and thereby enhance the stability of the clock.

Binding of CRY-proteins to the genome is not restricted to CLOCK-BMAL1 binding sites and extends to thousands of additional sites containing DNA-binding motifs for nuclear receptors (Koike et al., 2012; Takahashi, 2017). CRYs were reported to broadly interact with nuclear receptors and repress their transcriptional activity (Kriebs et al., 2017; Lamia et al., 2011). Whether CRYs also fulfill their inhibitory function through sequestering the transactivation domains of the hormone receptors and thereby regulate RNA Pol II pause release requires further studies.

TIP60 acetylates BMAL1 during the activation phase, but it is not clear by which mechanism BMAL1 is deacetylated during the repression phase. One possibility is a SIRT1-dependent deacetylation of BMAL1 (Nakahata et al., 2008). Whereby recent studies suggest that not BMAL1 but PER2 could be the direct substrate of SIRT1 (Asher et al., 2008; Foteinou et al., 2018). This finding is consistent with the minor circadian phenotype reported for SIRT1-deficiency at the tissue level (Chang and Guarente, 2013; Wang et al., 2016). PER proteins recruit a wide variety of chromatin modifiers and remodelers to the promoters of clock-controlled genes (Brown et al., 2005; Duong and Weitz, 2014; Duong et al., 2011; Kim et al., 2014; Padmanabhan et al., 2012). One of the chromatin modifiers is HDAC1 (Duong et al., 2011; Kim et al., 2014), that could directly deacetylate BMAL1 during the early repression phase of the circadian cycle (Koike et al., 2012). Acetylated BMAL1 could also be displaced from circadian promoters by PER proteins in a CRY-dependent manner (Chiou et al., 2016; Ye et al., 2011, 2014). Alternatively, promoter-associated acetylated BMAL1 could be subject to rapid proteolytic turnover (Stratmann et al., 2012) obviating the need for a specific deacetylase. A vast majority of strongly rhythmic CLOCK-BMAL1-target genes harbor a highly conserved tandem E-box motif (E1-E2) that engages CLOCK-BMAL1 cooperatively and favors strong binding and very precise, phase-specific gene expression (Rey et al., 2011; Shimomura et al., 2013). Increased abundance of acetylated BMAL1 at the E1-E2 motifs would recruit more BRD4-P-TEFb and allow efficient and synchronized pause release of RNA Pol II. Dbp, Perl, and Rev-Erba belong to this class of genes. This suggests that regulation of RNA Pol II pause release could extend to the entire class of these E-box 
controlled clock genes. These genes are synchronously expressed and would benefit from a precisely timed RNA Pol II pause release process. It is not clear, however, whether BRD4-P-TEFb mediated RNA Pol II pause release applies for other types of clock genes. Temporal analyses of RNA Pol II pause release at the transcriptome-wide scale will address these issues.

Taken together, this study demonstrates that the TIP60-mediated acetylation of BMAL1 links the transcriptional activators CLOCK-BMAL1 and RNA Pol II-mediated transcription of clock-controlled genes. Acetylation of BMAL1 leads to recruitment of BRD4-P-TEFb to circadian promoters and allows RNA Pol II pause release and productive elongation. CRY proteins prevent TIP60-mediated acetylation and thereby repress BRD4-P-TEFb recruitment and productive elongation of E-box-containing circadian transcripts. Hence, the positive and negative control of RNA Pol II pause release are an important element of the circadian clockwork and could allow a precise and synchronous expression of core clock-controlled genes and in this way also contribute to robustness of the circadian clock. This added layer of regulation could also provide a temporal window to integrate signals and possibly regulate the magnitude of transcriptional output. Robust and synchronous expression are the hallmarks of genes involved in stimulus-controlled pathways, whose transcription is also often regulated through Pol II pause release (Jonkers and Lis, 2015; Liu et al., 2015; Siegal and Rushlow, 2012). Uncovering the bromodomain-containing co-activator BRD4 as a main regulator of the circadian clock could provide new therapeutic strategies to treat circadian clockrelated disorders (Wang and Filippakopoulos, 2015). 


\section{Chapter 5: Additional Data}

In fibroblasts, but also in the SCN, deletion of TIP60 resulted in a nearly complete loss of rhythmic expression of numerous circadian genes suggesting that in these systems and for these genes TIP60 function is non-redundant. To extend the understanding of TIP60mediated RNA Pol II pause release, liver tissue was decided to be used for subsequent analysis. The mouse liver is a relatively large, homogeneous tissue that is not only easily accessible but also harbors a functional circadian clock that controls liver-specific gene expression. Numerous genome-wide studies have used this organ to reveal the molecular functions of various clock components and their temporal orchestration (Cho et al., 2012; Fang et al., 2014; Feng et al., 2011; Koike et al., 2012; Rey et al., 2011).

The majority of the experiments in this study were performed with fibroblasts, leaving open the question of whether the proposed mechanism of TIP60-mediated and CRY counteracting control of RNA Pol II pause release also occurs in other tissues, such as the liver. Although this study could show that acetylation of BAML1 occurs during the activation phase and is negatively regulated by CRY proteins in the liver, it remains unclear whether BMAL1 acetylation triggers BRD4-P-TEFb recruitment to E-box containing clock genes. Another open question is whether TIP60 also acetylates BMAL1 in the liver. In order to shed some light on this question, Tip $60^{f l f l}$ mice were crossed to Alb-CRE driver mice. Tip60 $0^{f l f l}$ Alb-Cre mice were viable, fertile and showed no obvious abnormalities. The genome-wide transcriptional analysis of the liver of these animals will not only make it possible to answer the above question, but also to uncover whether TIP60-mediated control of RNA Pol II pause release applies for all E-box controlled clock genes or only to a separate class of strongly rhythmic, phase specific CLOCK-BMAL1target genes that contain multiple E-boxes such as Dbp, Perl, and Rev-Erbo. Another limitation of the current study was the insufficient mapping of TIP60 to the genome. To overcome this problem and to investigate the temporal genome-wide binding of TIP60 to DNA, LSL-Tip60-FLAG transgenic mice were generated (Figure 9). These mice were then first mated to Tip $60^{f l f l}$ mice and subsequently crossed to Alb-CRE driver mice. Tip60 flffl $; L S L-T i p 60-F L A G ; A l b-C r e$ mice are deficient for TIP60 in the liver and instead express a C-terminal FLAG-tagged version of TIP60 in this tissue. These mice were viable, fertile and showed no obvious abnormalities. Temporal genome-wide ChIP-seq analysis of TIP $60^{\text {FLAG }}$ will uncover direct TIP60 target genes and contribute to the understanding of the on-chromatin competition of TIP60 and CRY proteins for BMAL1. 


\section{Chapter 6: References}

Acharya, D., Hainer, S.J., Yoon, Y., Wang, F., Bach, I., Rivera-Pérez, J.A., and Fazzio, T.G. (2017). KAT-Independent Gene Regulation by Tip60 Promotes ESC Self-Renewal but Not Pluripotency. Cell Rep. 19, 671-679.

Adli, M., and Bernstein, B.E. (2011). Whole-genome chromatin profiling from limited numbers of cells using nano-ChIP-seq. Nat. Protoc. 6, 1656-1668.

Aryal, R.P., Kwak, P.B., Tamayo, A.G., Gebert, M., Chiu, P.-L., Walz, T., and Weitz, C.J. (2017). Macromolecular Assemblies of the Mammalian Circadian Clock. Mol. Cell 67, 770-782.e6.

Asher, G., Gatfield, D., Stratmann, M., Reinke, H., Dibner, C., Kreppel, F., Mostoslavsky, R., Alt, F.W., and Schibler, U. (2008). SIRT1 regulates circadian clock gene expression through PER2 deacetylation. Cell 134, 317-328.

Balsalobre, A., Marcacci, L., and Schibler, U. (2000). Multiple signaling pathways elicit circadian gene expression in cultured Rat-1 fibroblasts. Curr. Biol. 10, 1291-1294.

Bararia, D., Trivedi, A.K., Zada, A.A.P., Greif, P.A., Mulaw, M.A., Christopeit, M., Hiddemann, W., Bohlander, S.K., and Behre, G. (2008). Proteomic identification of the MYST domain histone acetyltransferase TIP60 (HTATIP) as a co-activator of the myeloid transcription factor C/EBP $\alpha$. Leukemia 22, 800-807.

Bass, J., and Takahashi, J.S. (2010). Circadian integration of metabolism and energetics. Science 330, 1349-1354.

Bedrosian, T.A., Fonken, L.K., and Nelson, R.J. (2015). Endocrine Effects of Circadian Disruption. Annu. Rev. Physiol. 78, 109-131.

Beersma, D.G.M., and Gordijn, M.C.M. (2007). Circadian control of the sleep-wake cycle. Physiol. Behav. 90, 190-195.

Berndsen, C.E., Albaugh, B.N., Tan, S., and Denu, J.M. (2007). Catalytic Mechanism of a MYST Family Histone Acetyltransferase. Biochemistry 46, 623-629.

Boettiger, A.N., and Levine, M. (2009). Synchronous and stochastic patterns of gene activation in the Drosophila embryo. Science 325, 471-473.

Brown, S.A., Ripperger, J., Kadener, S., Fleury-Olela, F., Vilbois, F., Rosbash, M., and Schibler, U. (2005). PERIOD1-associated proteins modulate the negative limb of the mammalian circadian oscillator. Science 308, 693-696.

Buhr, E.D., Yoo, S.-H., and Takahashi, J.S. (2010). Temperature as a universal resetting cue for mammalian circadian oscillators. Science 330, 379-385.

Bunger, M.K., Wilsbacher, L.D., Moran, S.M., Clendenin, C., Radcliffe, L.A., Hogenesch, J.B., Simon, M.C., Takahashi, J.S., and Bradfield, C.A. (2000). Mop3 Is an Essential Component of the Master Circadian Pacemaker in Mammals. Cell 103, 10091017.

Busino, L., Bassermann, F., Maiolica, A., Lee, C., Nolan, P.M., Godinho, S.I.H., Draetta, G.F., and Pagano, M. (2007). SCFFbx13 Controls the Oscillation of the Circadian Clock by Directing the Degradation of Cryptochrome Proteins. Science 316, 900-904.

Challet, E. (2019). The circadian regulation of food intake. Nat. Rev. Endocrinol. 15, 393-405. 
Chang, H.-C., and Guarente, L. (2013). SIRT1 mediates central circadian control in the SCN by a mechanism that decays with aging. Cell 153, 1448-1460.

Chao, S.H., and Price, D.H. (2001). Flavopiridol inactivates P-TEFb and blocks most RNA polymerase II transcription in vivo. J. Biol. Chem. 276, 31793-31799.

Chao, S.H., Fujinaga, K., Marion, J.E., Taube, R., Sausville, E.A., Senderowicz, A.M., Peterlin, B.M., and Price, D.H. (2000). Flavopiridol inhibits P-TEFb and blocks HIV-1 replication. J. Biol. Chem. 275, 28345-28348.

Charvet, C., Wissler, M., Brauns-Schubert, P., Wang, S.-J., Tang, Y., Sigloch, F.C., Mellert, H., Brandenburg, M., Lindner, S.E., Breit, B., et al. (2011). Phosphorylation of Tip60 by GSK-3 Determines the Induction of PUMA and Apoptosis by p53. Mol. Cell 42, 584-596.

Chen, Z., Yoo, S.-H., Park, Y.-S., Kim, K.-H., Wei, S., Buhr, E., Ye, Z.-Y., Pan, H.-L., and Takahashi, J.S. (2012). Identification of diverse modulators of central and peripheral circadian clocks by high-throughput chemical screening. Proc. Natl. Acad. Sci. 109, 101-106.

Chiou, Y., Yang, Y., Rashid, N., Ye, R., Selby, C.P., and Sancar, A. (2016).

Mammalian Period represses and de-represses transcription by displacing CLOCK-

BMAL1 from promoters in a Cryptochrome-dependent manner. Proc. Natl. Acad. Sci.

U. S. A. 113, E6072-E6079.

Cho, H., Zhao, X., Hatori, M., Yu, R.T., Barish, G.D., Lam, M.T., Chong, L., DiTacchio, L., Atkins, A.R., Glass, C.K., et al. (2012). Regulation of circadian behaviour and metabolism by REV-ERB- $\alpha$ and REV-ERB- $\beta$. Nature 485, 123-127. Clinckemalie, L., Vanderschueren, D., Boonen, S., and Claessens, F. (2012). The hinge region in androgen receptor control. Mol. Cell. Endocrinol. 358, 1-8.

Core, L., and Adelman, K. (2019). Promoter-proximal pausing of RNA polymerase II: a nexus of gene regulation. Genes Dev. 33, 960-982.

Curtis, A.M., Seo, S., Westgate, E.J., Rudic, R.D., Smyth, E.M., Chakravarti, D., FitzGerald, G.A., and McNamara, P. (2004). Histone acetyltransferase-dependent chromatin remodeling and the vascular clock. J. Biol. Chem. 279, 7091-7097.

Damiola, F., Le Minh, N., Preitner, N., Kornmann, B., Fleury-Olela, F., and Schibler, U. (2000). Restricted feeding uncouples circadian oscillators in peripheral tissues from the central pacemaker in the suprachiasmatic nucleus. Genes Dev. 14, 2950-2961. DeBruyne, J.P., Noton, E., Lambert, C.M., Maywood, E.S., Weaver, D.R., and Reppert, S.M. (2006). A Clock Shock: Mouse CLOCK Is Not Required for Circadian Oscillator Function. Neuron 50, 465-477.

DeBruyne, J.P., Weaver, D.R., and Reppert, S.M. (2007). Peripheral circadian oscillators require CLOCK. Curr. Biol. 17, R538-R539.

Decker, P. V, Yu, D. Y., Iizuka, M., Qiu, Q., and Smith, M.M. (2008). Catalytic-site mutations in the MYST family histone Acetyltransferase Esa1. Genetics 178, 12091220.

Dibner, C., Schibler, U., and Albrecht, U. (2010). The mammalian circadian timing system: organization and coordination of central and peripheral clocks. Annu. Rev. Physiol. 72, 517-549.

Dimauro, I., Pearson, T., Caporossi, D., and Jackson, M.J. (2012). A simple protocol for the subcellular fractionation of skeletal muscle cells and tissue. BMC Res. Notes 5, 513. 
DiTacchio, L., Le, H.D., Vollmers, C., Hatori, M., Witcher, M., Secombe, J., and Panda, S. (2011). Histone Lysine Demethylase JARID1a Activates CLOCK-BMAL1 and Influences the Circadian Clock. Science 333, 1881-1885.

Doi, M., Hirayama, J., and Sassone-Corsi, P. (2006). Circadian Regulator CLOCK Is a Histone Acetyltransferase. Cell 125, 497-508.

Doyon, Y., and Côté, J. (2004). The highly conserved and multifunctional NuA4 HAT complex. Curr. Opin. Genet. Dev. 14, 147-154.

Duong, H.A., and Weitz, C.J. (2014). Temporal orchestration of repressive chromatin modifiers by circadian clock Period complexes. Nat. Struct. Mol. Biol. 21, 126-132.

Duong, H.A., Robles, M.S., Knutti, D., and Weitz, C.J. (2011). A molecular mechanism for circadian clock negative feedback. Science 332, 1436-1439.

Durkin, M.E., Qian, X., Popescu, N.C., and Lowy, D.R. (2013). Isolation of Mouse Embryo Fibroblasts. Bio-Protocol 3, e908.

Eick, D., and Geyer, M. (2013). The RNA Polymerase II Carboxy-Terminal Domain (CTD) Code. Chem. Rev. 113, 8456-8490.

Eide, E.J., Woolf, M.F., Kang, H., Woolf, P., Hurst, W., Camacho, F., Vielhaber, E.L., Giovanni, A., and Virshup, D.M. (2005). Control of mammalian circadian rhythm by CKI $\varepsilon$-regulated proteasome-mediated PER2 degradation. Mol. Cell. Biol. 25, 27952807.

Etchegaray, J., Lee, C., Wade, P.A., and Reppert, S.M. (2003). Rhythmic histone acetylation underlies transcription in the mammalian circadian clock. Nature 421, 177182.

Fang, B., Everett, L.J., Jager, J., Briggs, E., Armour, S.M., Feng, D., Roy, A., GerhartHines, Z., Sun, Z., and Lazar, M.A. (2014). Circadian enhancers coordinate multiple phases of rhythmic gene transcription in vivo. Cell 159, 1140-1152.

Feng, D., Liu, T., Sun, Z., Bugge, A., Mullican, S.E., Alenghat, T., Liu, X.S., and Lazar, M.A. (2011). A circadian rhythm orchestrated by histone deacetylase 3 controls hepatic lipid metabolism. Science 331, 1315-1319.

Fisher, J.B., Horst, A., Wan, T., Kim, M.S., Auchampach, J., and Lough, J. (2016). Depletion of Tip60 from in vivo cardiomyocytes increases myocyte density, followed by cardiac dysfunction, myocyte fallout and lethality. PLoS One 11, e0164855.

Foteinou, P.T., Venkataraman, A., Francey, L.J., Anafi, R.C., Hogenesch, J.B., Doyle, F.J., and III (2018). Computational and experimental insights into the circadian effects of SIRT1. Proc. Natl. Acad. Sci. U. S. A. 115, 11643-11648.

Frank, S.R., Parisi, T., Taubert, S., Fernandez, P., Fuchs, M., Chan, H.-M., Livingston, D.M., and Amati, B. (2003). MYC recruits the TIP60 histone acetyltransferase complex to chromatin. EMBO Rep. 4, 575-580.

Gachon, F., Fonjallaz, P., Damiola, F., Gos, P., Kodama, T., Zakany, J., Duboule, D., Petit, B., Tafti, M., and Schibler, U. (2004). The loss of circadian PAR bZip transcription factors results in epilepsy. Genes Dev. 18, 1397-1412.

Gallego, M., and Virshup, D.M. (2007). Post-translational modifications regulate the ticking of the circadian clock. Nat. Rev. Mol. Cell Biol. 8, 139-148.

Gaucher, J., Montellier, E., and Sassone-Corsi, P. (2018). Molecular Cogs: Interplay between Circadian Clock and Cell Cycle. Trends Cell Biol. 28, 368-379.

Gauger, M.A., and Sancar, A. (2005). Cryptochrome, Circadian Cycle, Cell Cycle 
Checkpoints, and Cancer. Cancer Res. 65, 6828-6834.

Gekakis, N., Staknis, D., Nguyen, H.B., Davis, F.C., Wilsbacher, L.D., King, D.P., Takahashi, J.S., and Weitz, C.J. (1998). Role of the CLOCK protein in the mammalian circadian mechanism. Science 280, 1564-1569.

Gerstner, J.R., and Yin, J.C. (2010). Circadian rhythms and memory formation. Nat. Rev. Neurosci. 11, 577-588.

Gévry, N., Chan, H.M., Laflamme, L., Livingston, D.M., and Gaudreau, L. (2007). p21 transcription is regulated by differential localization of histone H2A.Z. Genes Dev. 21, 1869-1881.

Ghobashi, A.H., and Kamel, M.A. (2018). Tip60: updates. J. Appl. Genet. 59, 161-168. Gilchrist, D.A., Dos Santos, G., Fargo, D.C., Xie, B., Gao, Y., Li, L., and Adelman, K. (2010). Pausing of RNA Polymerase II Disrupts DNA-Specified Nucleosome Organization to Enable Precise Gene Regulation. Cell 143, 540-551.

Gilchrist, D.A., Fromm, G., dos Santos, G., Pham, L.N., Mcdaniel, I.E., Burkholder, A., Fargo, D.C., and Adelman, K. (2012). Regulating the regulators: the pervasive effects of Pol II pausing on stimulus-responsive gene networks. Genes Dev. 26, 933-944.

Godinho, S.I., Maywood, E.S., Shaw, L., Tucci, V., Barnard, A.R., Busino, L., Pagano, M., Kendall, R., Quwailid, M.M., Romero, M.R., et al. (2007). The after-hours mutant reveals a role for Fbxl3 in determining mammalian circadian period. Science 316, 897900.

Green, C.B., Takahashi, J.S., and Bass, J. (2008). The meter of metabolism. Cell 134, 728-742.

Guillaumond, F., Dardente, H., Giguère, V., and Cermakian, N. (2005). Differential Control of Bmal1 Circadian Transcription by REV-ERB and ROR Nuclear Receptors. J. Biol. Rhythms 20, 391-403.

Hampsey, M., Singh, B.N., Ansari, A., Lainé, J.-P., and Krishnamurthy, S. (2011). Control of eukaryotic gene expression: Gene loops and transcriptional memory. Adv. Enzyme Regul. 51, 118-125.

Harlen, K.M., and Churchman, L.S. (2017). The code and beyond: transcription regulation by the RNA polymerase II carboxy-terminal domain. Nat. Rev. Mol. Cell Biol. 18, 263-273.

Hayes, O., Ramos, B., Rodríguez, L.L., Aguilar, A., Badía, T., and Castro, F.O. (2005). Cell confluency is as efficient as serum starvation for inducing arrest in the G0/G1 phase of the cell cycle in granulosa and fibroblast cells of cattle. Anim. Reprod. Sci. 87, 181-192.

Henrich, V.C., Vogtli, M.E., Antoniewski, C., Spindler-Barth, M., Przibilla, S., Noureddine, M., and Lezzi, M. (2000). Widespread recombinase expression using FLPeR (flipper) mice. Genesis 28, 106-110.

Hirano, A., Yumimoto, K., Tsunematsu, R., Matsumoto, M., Oyama, M., Kozuka-Hata, H., Nakagawa, T., Lanjakornsiripan, D., Nakayama, K.I., and Fukada, Y. (2013). FBXL21 Regulates Oscillation of the Circadian Clock through Ubiquitination and Stabilization of Cryptochromes. Cell 152, 1106-1118.

Hirayama, J., Sahar, S., Grimaldi, B., Tamaru, T., Takamatsu, K., Nakahata, Y., and Sassone-Corsi, P. (2007). CLOCK-mediated acetylation of BMAL1 controls circadian function. Nature 450, 1086-1090. 
Hirota, T., Lewis, W.G., Liu, A.C., Lee, J.W., Schultz, P.G., and Kay, S.A. (2008). A chemical biology approach reveals period shortening of the mammalian circadian clock by specific inhibition of GSK-3. Proc. Natl. Acad. Sci. 105, 20746-20751.

Hirota, T., Lee, J.W., Lewis, W.G., Zhang, E.E., Breton, G., Liu, X., Garcia, M., Peters, E.C., Etchegaray, J.-P., Traver, D., et al. (2010). High-Throughput Chemical Screen Identifies a Novel Potent Modulator of Cellular Circadian Rhythms and Reveals CKI $\alpha$ as a Clock Regulatory Kinase. PLoS Biol. 8, e1000559.

Hirota, T., Lee, J.W., St John, P.C., Sawa, M., Iwaisako, K., Noguchi, T., Pongsawakul, P.Y., Sonntag, T., Welsh, D.K., Brenner, D.A., et al. (2012). Identification of small molecule activators of cryptochrome. Science 337, 1094-1097.

van der Horst, G.T.J., Muijtjens, M., Kobayashi, K., Takano, R., Kanno, S., Takao, M., de Wit, J., Verkerk, A., Eker, A.P., van Leenen, D., et al. (1999). Mammalian Cry1 and Cry2 are essential for maintenance of circadian rhythms. Nature 398, 627-630.

Hosoda, H., Kato, K., Asano, H., Ito, M., Kato, H., Iwamoto, T., Suzuki, A., Masushige, S., and Kida, S. (2009). CBP/p300 is a cell type-specific modulator of CLOCK/BMAL1-mediated transcription. Mol. Brain 2, 34.

Hu, Y., Fisher, J.B., Koprowski, S., McAllister, D., Kim, M.S., and Lough, J. (2009). Homozygous disruption of the Tip60 gene causes early embryonic lethality. Dev. Dyn. 238, 2912-2921.

Huang, B., Yang, X.-D., Zhou, M.-M., Ozato, K., and Chen, L.-F. (2009). Brd4 coactivates transcriptional activation of NF-kappaB via specific binding to acetylated RelA. Mol. Cell. Biol. 29, 1375-1387.

Husse, J., Zhou, X., Shostak, A., Oster, H., and Eichele, G. (2011). Synaptotagmin10Cre, a Driver to Disrupt Clock Genes in the SCN. J. Biol. Rhythms 26, 379-389. Isojima, Y., Nakajima, M., Ukai, H., Fujishima, H., Yamada, R.G., Masumoto, K., Kiuchi, R., Ishida, M., Ukai-Tadenuma, M., Minami, Y., et al. (2009).

CKIepsilon/delta-dependent phosphorylation is a temperature-insensitive, perioddetermining process in the mammalian circadian clock. Proc. Natl. Acad. Sci. U. S. A. 106, 15744-15749.

Jacquet, K., Fradet-Turcotte, A., Avvakumov, N., Lambert, J.-P., Roques, C., Pandita, R.K., Paquet, E., Herst, P., Gingras, A.-C., Pandita, T.K., et al. (2016). The TIP60 Complex Regulates Bivalent Chromatin Recognition by 53BP1 through Direct H4K20me Binding and H2AK15 Acetylation. Mol. Cell 62, 409-421.

Jonkers, I., and Lis, J.T. (2015). Getting up to speed with transcription elongation by RNA polymerase II. Nat. Rev. Mol. Cell Biol. 16, 167-177.

Jonkers, I., Kwak, H., and Lis, J.T. (2014). Genome-wide dynamics of Pol II elongation and its interplay with promoter proximal pausing, chromatin, and exons. Elife 3, e02407.

Jud, C., Schmutz, I., Hampp, G., Oster, H., and Albrecht, U. (2005). A guideline for analyzing circadian wheel-running behavior in rodents under different lighting conditions. Biol. Proced. Online 7, 101-116.

Judes, G., Rifaï, K., Ngollo, M., Daures, M., Bignon, Y.-J., Penault-Llorca, F., and Bernard-Gallon, D. (2015). A bivalent role of TIP60 histone acetyl transferase in human cancer. Epigenomics 7, 1351-1363.

Katada, S., and Sassone-Corsi, P. (2010). The histone methyltransferase MLL1 permits 
the oscillation of circadian gene expression. Nat. Struct. Mol. Biol. 17, 1414-1421. Khan, S.K., Xu, H., Ukai-Tadenuma, M., Burton, B., Wang, Y., Ueda, H.R., and Liu, A.C. (2012). Identification of a novel cryptochrome differentiating domain required for feedback repression in circadian clock function. J. Biol. Chem. 287, 25917-25926. Kim, J.-W., Jang, S.-M., Kim, C.-H., An, J.-H., Kang, E.-J., and Choi, K.-H. (2012). New molecular bridge between RelA/p65 and NF- $\kappa$ B target genes via histone acetyltransferase TIP60 cofactor. J. Biol. Chem. 287, 7780-7791.

Kim, J.Y., Kwak, P.B., Gebert, M., Duong, H.A., and Weitz, C.J. (2015). Purification and Analysis of PERIOD Protein Complexes of the Mammalian Circadian Clock. Methods Enzymol. 551, 197-210.

Kim, J.Y.Y., Kwak, P.B.B., and Weitz, C.J.J. (2014). Specificity in Circadian Clock Feedback from Targeted Reconstitution of the NuRD Corepressor. Mol. Cell 56, 738748.

King, D.P., Zhao, Y., Sangoram, A.M., Wilsbacher, L.D., Tanaka, M., Antoch, M.P., Steeves, T.D.., Vitaterna, M.H., Kornhauser, J.M., Lowrey, P.L., et al. (1997).

Positional Cloning of the Mouse Circadian Clock Gene. Cell 89, 641-653.

Kiyohara, Y.B., Tagao, S., Tamanini, F., Morita, A., Sugisawa, Y., Yasuda, M., Yamanaka, I., Ueda, H.R., van der Horst, G.T.J., Kondo, T., et al. (2006). The BMAL1 C terminus regulates the circadian transcription feedback loop. Proc. Natl. Acad. Sci. U. S. A. 103, 10074-10079.

Koike, N., Yoo, S.-H., Huang, H.-C., Kumar, V., Lee, C., Kim, T.-K., and Takahashi, J.S. (2012). Transcriptional architecture and chromatin landscape of the core circadian clock in mammals. Science 338, 349-354.

Korf, H.-W., and von Gall, C. (2013). Circadian Physiology. In Neuroscience in the 21st Century, (New York, NY: Springer New York), pp. 1813-1845.

Kornmann, B., Schaad, O., Reinke, H., Saini, C., and Schibler, U. (2007a). Regulation of circadian gene expression in liver by systemic signals and hepatocyte oscillators. Cold Spring Harb. Symp. Quant. Biol. 72, 319-330.

Kornmann, B., Schaad, O., Bujard, H., Takahashi, J.S., and Schibler, U. (2007b). System-Driven and Oscillator-Dependent Circadian Transcription in Mice with a Conditionally Active Liver Clock. PLoS Biol. 5, e34.

Koronowski, K.B., Kinouchi, K., Welz, P.-S., Smith, J.G., Zinna, V.M., Shi, J., Samad, M., Chen, S., Magnan, C.N., Kinchen, J.M., et al. (2019). Defining the Independence of the Liver Circadian Clock. Cell 177, 1448-1462.e14.

Kriebs, A., Jordan, S.D., Soto, E., Henriksson, E., Sandate, C.R., Vaughan, M.E., Chan, A.B., Duglan, D., Papp, S.J., Huber, A.-L., et al. (2017). Circadian repressors CRY1 and CRY2 broadly interact with nuclear receptors and modulate transcriptional activity. Proc. Natl. Acad. Sci. U. S. A. 114, 8776-8781.

Kume, K., Zylka, M.J., Sriram, S., Shearman, L.P., Weaver, D.R., Jin, X., Maywood, E.S., Hastings, M.H., and Reppert, S.M. (1999). mCRY1 and mCRY2 are essential components of the negative limb of the circadian clock feedback loop. Cell 98, 193205.

Kusch, T., Florens, L., MacDonald, W.H., Swanson, S.K., Glaser, R.L., Yates, J.R., Abmayr, S.M., Washburn, M.P., and Workman, J.L. (2004). Acetylation by Tip60 Is Required for Selective Histone Variant Exchange at DNA Lesions. Science 306, 2084 
2087.

Lagha, M., Bothma, J.P., Esposito, E., Ng, S., Stefanik, L., Tsui, C., Johnston, J., Chen, K., Gilmour, D.S., Zeitlinger, J., et al. (2013). Paused Pol II coordinates tissue morphogenesis in the drosophila embryo. Cell 153, 976-987.

Lamia, K.A., Sachdeva, U.M., DiTacchio, L., Williams, E.C., Alvarez, J.G., Egan, D.F., Vasquez, D.S., Juguilon, H., Panda, S., Shaw, R.J., et al. (2009). AMPK regulates the circadian clock by cryptochrome phosphorylation and degradation. Science 326, 437440.

Lamia, K.A., Papp, S.J., Yu, R.T., Barish, G.D., Uhlenhaut, N.H., Jonker, J.W., Downes, M., and Evans, R.M. (2011). Cryptochromes mediate rhythmic repression of the glucocorticoid receptor. Nature 480, 552-556.

Lande-Diner, L., Boyault, C., Kim, J.Y., and Weitz, C.J. (2013). A positive feedback loop links circadian clock factor CLOCK-BMAL1 to the basic transcriptional machinery. Proc. Natl. Acad. Sci. U. S. A. 110, 16021-16026.

Lee, C., Etchegaray, J.-P., Cagampang, F.R.A., Loudon, A.S.I., and Reppert, S.M. (2001). Posttranslational Mechanisms Regulate the Mammalian Circadian Clock. Cell 107, 855-867.

Lee, H., Chen, R., Lee, Y., Yoo, S., and Lee, C. (2009). Essential roles of CKI $\delta$ and CKIE in the mammalian circadian clock. Proc. Natl. Acad. Sci. 106, 21359-21364.

Lee, Y., Lee, J., Kwon, I., Nakajima, Y., Ohmiya, Y., Son, G.H., Lee, K.H., and Kim, K. (2010). Coactivation of the CLOCK-BMAL1 complex by CBP mediates resetting of the circadian clock. J. Cell Sci. 123, 3547-3557.

Lin, C., Garrett, A.S., de Kumar, B., Smith, E.R., Gogol, M., Seidel, C., Krumlauf, R., and Shilatifard, A. (2011). Dynamic transcriptional events in embryonic stem cells mediated by the super elongation complex (SEC). Genes Dev. 25, 1486-1498.

Lin, C.Y., Lovén, J., Rahl, P.B., Paranal, R.M., Burge, C.B., Bradner, J.E., Lee, T.I., and Young, R.A. (2012). Transcriptional amplification in tumor cells with elevated cMyc. Cell 151, 56-67.

Lin, R., Mo, Y., Zha, H., Qu, Z., Xie, P., Zhu, Z.-J., Xu, Y., Xiong, Y., and Guan, K.-L. (2017). CLOCK Acetylates ASS1 to Drive Circadian Rhythm of Ureagenesis. Mol. Cell 68, 198-209.e6.

Liu, A.C., Welsh, D.K., Ko, C.H., Tran, H.G., Zhang, E.E., Priest, A.A., Buhr, E.D., Singer, O., Meeker, K., Verma, I.M., et al. (2007). Intercellular coupling confers robustness against mutations in the SCN circadian clock network. Cell 129, 605-616. Liu, A.C., Tran, H.G., Zhang, E.E., Priest, A.A., Welsh, D.K., and Kay, S.A. (2008). Redundant function of REV-ERB $\alpha$ and $\beta$ and non-essential role for Bmall cycling in transcriptional regulation of intracellular circadian rhythms. PLoS Genet. 4, e1000023. Liu, X., Kraus, W.L., and Bai, X. (2015). Ready, pause, go: Regulation of RNA polymerase II pausing and release by cellular signaling pathways. Trends Biochem. Sci. 40, 516-525.

Le Martelot, G., Canella, D., Symul, L., Migliavacca, E., Gilardi, F., Liechti, R., Martin, O., Harshman, K., Delorenzi, M., Desvergne, B., et al. (2012). Genome-Wide RNA Polymerase II Profiles and RNA Accumulation Reveal Kinetics of Transcription and Associated Epigenetic Changes During Diurnal Cycles. PLoS Biol. 10, e1001442. Melters, D.P., Nye, J., Zhao, H., and Dalal, Y. (2015). Chromatin Dynamics in Vivo: A 
Game of Musical Chairs. Genes (Basel). 6, 751-776.

Méndez, J., and Stillman, B. (2000). Chromatin association of human origin recognition complex, cdc6, and minichromosome maintenance proteins during the cell cycle: assembly of prereplication complexes in late mitosis. Mol. Cell. Biol. 20, 8602-8612. Mendlewicz, J. (2009). Disruption of the Circadian Timing Systems. CNS Drugs 23, $15-26$.

Menet, J.S., Rodriguez, J., Abruzzi, K.C., and Rosbash, M. (2012). Nascent-Seq reveals novel features of mouse circadian transcriptional regulation. Elife 1, e00011.

Menet, J.S., Pescatore, S., and Rosbash, M. (2014). CLOCK:BMAL1 is a pioneer-like transcription factor. Genes Dev. 28, 8-13.

Mitsui, S., Yamaguchi, S., Matsuo, T., Ishida, Y., and Okamura, H. (2001).

Antagonistic role of E4BP4 and PAR proteins in the circadian oscillatory mechanism. Genes Dev. 15, 995-1006.

Mizuguchi, G., Shen, X., Landry, J., Wu, W.-H., Sen, S., and Wu, C. (2004). ATPdriven exchange of histone $\mathrm{H} 2 \mathrm{AZ}$ variant catalyzed by SWR1 chromatin remodeling complex. Science 303, 343-348.

Moore, R.Y., and Eichler, V.B. (1972). Loss of a circadian adrenal corticosterone rhythm following suprachiasmatic lesions in the rat. Brain Res. 42, 201-206.

Mure, L.S., Le, H.D., Benegiamo, G., Chang, M.W., Rios, L., Jillani, N., Ngotho, M., Kariuki, T., Dkhissi-Benyahya, O., Cooper, H.M., et al. (2018). Diurnal transcriptome atlas of a primate across major neural and peripheral tissues. Science (80-. ). 359, eaao0318.

Musiek, E.S., and Holtzman, D.M. (2016). Mechanisms linking circadian clocks, sleep, and neurodegeneration. Science 354, 1004-1008.

Nagoshi, E., Saini, C., Bauer, C., Laroche, T., Naef, F., and Schibler, U. (2004).

Circadian gene expression in individual fibroblasts: Cell-autonomous and self-sustained oscillators pass time to daughter cells. Cell 119, 693-705.

Nakahata, Y., Kaluzova, M., Grimaldi, B., Sahar, S., Hirayama, J., Chen, D., Guarente, L.P., and Sassone-Corsi, P. (2008). The NAD+-Dependent Deacetylase SIRT1

Modulates CLOCK-Mediated Chromatin Remodeling and Circadian Control. Cell 134, 329-340.

Nelson, J.D., Denisenko, O., and Bomsztyk, K. (2006). Protocol for the fast chromatin immunoprecipitation (ChIP) method. Nat. Protoc. 1, 179-185.

Oishi, K., Miyazaki, K., Kadota, K., Kikuno, R., Nagase, T., Atsumi, G., Ohkura, N., Azama, T., Mesaki, M., Yukimasa, S., et al. (2003). Genome-wide expression analysis of mouse liver reveals CLOCK-regulated circadian output genes. J. Biol. Chem. 278, 41519-41527.

Oster, H., Baeriswyl, S., van der Horst, G.T.J., and Albrecht, U. (2003). Loss of circadian rhythmicity in aging mPer1-/- mCry2-/- mutant mice. Genes Dev. 17, 13661379.

Oster, H., Damerow, S., Kiessling, S., Jakubcakova, V., Abraham, D., Tian, J., Hoffmann, M.W., and Eichele, G. (2006). The circadian rhythm of glucocorticoids is regulated by a gating mechanism residing in the adrenal cortical clock. Cell Metab. 4, 163-173.

Padmanabhan, K., Robles, M.S., Westerling, T., and Weitz, C.J. (2012). Feedback 
Regulation of Transcriptional Termination by the Mammalian Circadian Clock PERIOD Complex. Science 337, 599-602.

Panda, S. (2016). Circadian physiology of metabolism. Science 354, 317-322.

Papazyan, R., Zhang, Y., and Lazar, M.A. (2016). Genetic and epigenomic mechanisms of mammalian circadian transcription. Nat. Struct. Mol. Biol. 23, 1045-1052.

Partch, C.L., Shields, K.F., Thompson, C.L., Selby, C.P., and Sancar, A. (2006). Posttranslational regulation of the mammalian circadian clock by cryptochrome and protein phosphatase 5. Proc. Natl. Acad. Sci. U. S. A. 103, 10467-10472.

Patel, J.H., Du, Y., Ard, P.G., Phillips, C., Carella, B., Chen, C.-J., Rakowski, C., Chatterjee, C., Lieberman, P.M., Lane, W.S., et al. (2004). The c-MYC oncoprotein is a substrate of the acetyltransferases hGCN5/PCAF and TIP60. Mol. Cell. Biol. 24, 10826-10834.

Patke, A., Murphy, P.J., Onat, O.E., Krieger, A.C., Özçelik, T., Campbell, S.S., and Young, M.W. (2017). Mutation of the Human Circadian Clock Gene CRY1 in Familial Delayed Sleep Phase Disorder. Cell 169, 203-215.e13.

Pittendrigh, C.S. (1993). Temporal Organization: Reflections of a Darwinian ClockWatcher. Annu. Rev. Physiol. 55, 17-54.

Plautz, J.D., Kaneko, M., Hall, J.C., Kay, S.A., and Menaker, M. (1997). Independent Photoreceptive Circadian Clocks Throughout Drosophila. Science (80-. ). 278, 16321635.

Porrua, O., and Libri, D. (2015). Transcription termination and the control of the transcriptome: why, where and how to stop. Nat. Rev. Mol. Cell Biol. 16, 190-202. Postic, C., Shiota, M., Niswender, K.D., Jetton, T.L., Chen, Y., Moates, J.M., Shelton, K.D., Lindner, J., Cherrington, A.D., and Magnuson, M.A. (1999). Dual roles for glucokinase in glucose homeostasis as determined by liver and pancreatic beta cellspecific gene knock-outs using Cre recombinase. J. Biol. Chem. 274, 305-315.

Pradhan, S.K., Su, T., Yen, L., Jacquet, K., Huang, C., Côté, J., Kurdistani, S.K., and Carey, M.F. (2016). EP400 Deposits H3.3 into Promoters and Enhancers during Gene Activation. Mol. Cell 61, 27-38.

Preitner, N., Damiola, F., Luis-Lopez-Molina, Zakany, J., Duboule, D., Albrecht, U., and Schibler, U. (2002). The orphan nuclear receptor REV-ERB $\alpha$ controls circadian transcription within the positive limb of the mammalian circadian oscillator. Cell 110, 251-260.

Preußner, M., and Heyd, F. (2016). Post-transcriptional control of the mammalian circadian clock: implications for health and disease. Pflügers Arch. - Eur. J. Physiol. 468, 983-991.

Rahl, P.B., Lin, C.Y., Seila, A.C., Flynn, R.A., McCuine, S., Burge, C.B., Sharp, P.A., and Young, R.A. (2010). C-Myc regulates transcriptional pause release. Cell 141, 432445 .

Ramezani, A., and Hawley, R.G. (2002). Generation of HIV-1-based lentiviral vector particles. In Current Protocols in Molecular Biology, p. Unit 16.22.

Ravens, S., Yu, C., Ye, T., Stierle, M., and Tora, L. (2015). Tip60 complex binds to active Pol II promoters and a subset of enhancers and co-regulates the c-Myc network in mouse embryonic stem cells. Epigenetics Chromatin 8, 45.

Reddy, T.E., Pauli, F., Sprouse, R.O., Neff, N.F., Newberry, K.M., Garabedian, M.J., 
and Myers, R.M. (2009). Genomic determination of the glucocorticoid response reveals unexpected mechanisms of gene regulation. Genome Res. 19, 2163-2171.

Reddy, T.E., Gertz, J., Crawford, G.E., Garabedian, M.J., and Myers, R.M. (2012). The hypersensitive glucocorticoid response specifically regulates period 1 and expression of circadian genes. Mol. Cell. Biol. 32, 3756-3767.

Refinetti, R. (2015). Comparison of light, food, and temperature as environmental synchronizers of the circadian rhythm of activity in mice. J. Physiol. Sci. 65, 359-366.

Reid, K., and Zee, P. (2009). Circadian Rhythm Disorders. Semin. Neurol. 29, 393-405. Reischl, S., Vanselow, K., Westermark, P.O., Thierfelder, N., Maier, B., Herzel, H., and Kramer, A. (2007). Beta-TrCP1-mediated degradation of PERIOD2 is essential for circadian dynamics. J. Biol. Rhythms 22, 375-386.

Rey, G., Cesbron, F., Rougemont, J., Reinke, H., Brunner, M., and Naef, F. (2011). Genome-wide and phase-specific DNA-binding rhythms of BMAL1 control circadian output functions in mouse liver. PLoS Biol. 9, e1000595.

Ripperger, J.A., and Schibler, U. (2006). Rhythmic CLOCK-BMAL1 binding to multiple E-box motifs drives circadian Dbp transcription and chromatin transitions. Nat. Genet. 38, 369-374.

Rosensweig, C., and Green, C.B. (2018). Periodicity, repression, and the molecular architecture of the mammalian circadian clock. Eur. J. Neurosci. ejn.14254.

Sainsbury, S., Bernecky, C., and Cramer, P. (2015). Structural basis of transcription initiation by RNA polymerase II. Nat. Rev. Mol. Cell Biol. 16, 129-143.

Sato, T.K., Yamada, R.G., Ukai, H., Baggs, J.E., Miraglia, L.J., Kobayashi, T.J., Welsh, D.K., Kay, S.A., Ueda, H.R., and Hogenesch, J.B. (2006). Feedback repression is required for mammalian circadian clock function. Nat. Genet. 38, 312-319.

Scheiermann, C., Kunisaki, Y., and Frenette, P.S. (2013). Circadian control of the immune system. Nat. Rev. Immunol. 13, 190-198.

Schibler, U., Gotic, I., Saini, C., Gos, P., Curie, T., Emmenegger, Y., Sinturel, F., Gosselin, P., Gerber, A., Fleury-Olela, F., et al. (2015). Clock-Talk: Interactions between Central and Peripheral Circadian Oscillators in Mammals. Cold Spring Harb. Symp. Quant. Biol. 80, 223-232.

Schwenk, F., Baron, U., and Rajewsky, K. (1995). A cre-transgenic mouse strain for the ubiquitous deletion of loxP-flanked gene segments including deletion in germ cells. Nucleic Acids Res. 23, 5080-5081.

Shandilya, J., and Roberts, S.G.E. (2012). The transcription cycle in eukaryotes: From productive initiation to RNA polymerase II recycling. Biochim. Biophys. Acta - Gene Regul. Mech. 1819, 391-400.

Shearman, L.P., Sriram, S., Weaver, D.R., Maywood, E.S., Chaves, I., Zheng, B., Kume, K., Lee, C.C., van der Horst, G.T., Hastings, M.H., et al. (2000). Interacting molecular loops in the mammalian circadian clock. Science 288, 1013-1019.

Shi, J., and Vakoc, C.R. (2014). The Mechanisms behind the Therapeutic Activity of BET Bromodomain Inhibition. Mol. Cell 54, 72-736.

Shi, J., Wang, Y., Zeng, L., Wu, Y., Deng, J., Zhang, Q., Lin, Y., Li, J., Kang, T., Tao, M., et al. (2014). Disrupting the Interaction of BRD4 with Diacetylated Twist

Suppresses Tumorigenesis in Basal-like Breast Cancer. Cancer Cell 25, 210-225.

Shimomura, K., Kumar, V., Koike, N., Kim, T.K., Chong, J., Buhr, E.D., Whiteley, 
A.R., Low, S.S., Omura, C., Fenner, D., et al. (2013). Usf1, a suppressor of the circadian Clock mutant, reveals the nature of the DNA-binding of the CLOCK:BMAL1 complex in mice. Elife 2, e00426.

Shirogane, T., Jin, J., Ang, X.L., and Harper, J.W. (2005). SCFß-TRCP controls Clockdependent transcription via casein kinase 1-dependent degradation of the mammalian period-1 (Per1) protein. J. Biol. Chem. 280, 26863-26872.

Siegal, M.L., and Rushlow, C. (2012). Pausing on the Path to Robustness. Dev. Cell 22, 905-906.

Siepka, S.M., Yoo, S.H., Park, J., Song, W., Kumar, V., Hu, Y., Lee, C., and Takahashi, J.S. (2007). Circadian Mutant Overtime Reveals F-box Protein FBXL3 Regulation of Cryptochrome and Period Gene Expression. Cell 129, 1011-1023.

Sobel, J.A., Krier, I., Andersin, T., Raghav, S., Canella, D., Gilardi, F., Kalantzi, A.S., Rey, G., Weger, B., Gachon, F., et al. (2017). Transcriptional regulatory logic of the diurnal cycle in the mouse liver. PLOS Biol. 15, e2001069.

Spengler, M.L., Kuropatwinski, K.K., Schumer, M., and Antoch, M. (2009). A serine cluster mediates BMAL1-dependent CLOCK phosphorylation and degradation. Cell Cycle 8, 4138-4146.

Stephan, F.K., and Zucker, I. (1972). Circadian rhythms in drinking behavior and locomotor activity of rats are eliminated by hypothalamic lesions. Proc. Natl. Acad. Sci. U. S. A. 69, 1583-1586.

Storch, K.-F., Lipan, O., Leykin, I., Viswanathan, N., Davis, F.C., Wong, W.H., and Weitz, C.J. (2002). Extensive and divergent circadian gene expression in liver and heart. Nature 417, 78-83.

Stratmann, M., Stadler, F., Tamanini, F., van der Horst, G.T.J., and Ripperger, J.A. (2010). Flexible phase adjustment of circadian albumin D site-binding protein (Dbp) gene expression by CRYPTOCHROME1. Genes Dev. 24, 1317-1328.

Stratmann, M., Suter, D.M., Molina, N., Naef, F., and Schibler, U. (2012). Circadian Dbp Transcription Relies on Highly Dynamic BMAL1-CLOCK Interaction with E Boxes and Requires the Proteasome. Mol. Cell 48, 277-287.

Sykes, S.M., Mellert, H.S., Holbert, M.A., Li, K., Marmorstein, R., Lane, W.S., and McMahon, S.B. (2006). Acetylation of the p53 DNA-Binding Domain Regulates Apoptosis Induction. Mol. Cell 24, 841-851.

Takahashi, J.S. (2017). Transcriptional architecture of the mammalian circadian clock. Nat. Rev. Genet. 18, 164-179.

Takahata, S., Ozaki, T., Mimura, J., Kikuchi, Y., Sogawa, K., and Fujii-Kuriyama, Y. (2000). Transactivation mechanisms of mouse clock transcription factors, mClock and mArnt3. Genes to Cells 5, 739-747.

Takeda, Y., Jothi, R., Birault, V., and Jetten, A.M. (2012). ROR $\gamma$ directly regulates the circadian expression of clock genes and downstream targets in vivo. Nucleic Acids Res. 40, 8519-8535.

Tang, Y., Luo, J., Zhang, W., and Gu, W. (2006). Tip60-Dependent Acetylation of p53 Modulates the Decision between Cell-Cycle Arrest and Apoptosis. Mol. Cell 24, $827-$ 839.

Taubert, S., Gorrini, C., Frank, S.R., Parisi, T., Fuchs, M., Chan, H.-M., Livingston, D.M., and Amati, B. (2004). E2F-dependent histone acetylation and recruitment of the 
Tip60 acetyltransferase complex to chromatin in late G1. Mol. Cell. Biol. 24, 45464556.

Toh, K.L., Jones, C.R., He, Y., Eide, E.J., Hinz, W.A., Virshup, D.M., Ptácek, L.J., and $\mathrm{Fu}$, Y.H. (2001). An hPer2 phosphorylation site mutation in familial advanced sleep phase syndrome. Science 291, 1040-1043.

Ueda, H.R., Hayashi, S., Chen, W., Sano, M., Machida, M., Shigeyoshi, Y., Iino, M., and Hashimoto, S. (2005). System-level identification of transcriptional circuits underlying mammalian circadian clocks. Nat. Genet. 37, 187-192.

Ukai-Tadenuma, M., Yamada, R.G., Xu, H., Ripperger, J.A., Liu, A.C., and Ueda, H.R. (2011). Delay in Feedback Repression by Cryptochrome 1 Is Required for Circadian Clock Function. Cell 144, 268-281.

Valekunja, U.K., Edgar, R.S., Oklejewicz, M., van der Horst, G.T.J., O’Neill, J.S., Tamanini, F., Turner, D.J., and Reddy, A.B. (2013). Histone methyltransferase MLL3 contributes to genome-scale circadian transcription. Proc. Natl. Acad. Sci. U. S. A. 110, 1554-1559.

Verlande, A., and Masri, S. (2019). Circadian Clocks and Cancer: Timekeeping Governs Cellular Metabolism. Trends Endocrinol. Metab.

Vitaterna, M.H., Selby, C.P., Todo, T., Niwa, H., Thompson, C., Fruechte, E.M., Hitomi, K., Thresher, R.J., Ishikawa, T., Miyazaki, J., et al. (1999). Differential regulation of mammalian Period genes and circadian rhythmicity by cryptochromes 1 and 2. Proc. Natl. Acad. Sci. 96, 12114-12119.

Vollmers, C., Schmitz, R.J., Nathanson, J., Yeo, G., Ecker, J.R., and Panda, S. (2012).

Circadian oscillations of protein-coding and regulatory RNAs in a highly dynamic mammalian liver epigenome. Cell Metab. 16, 833-845.

Wang, C.Y., and Filippakopoulos, P. (2015). Beating the odds: BETs in disease. Trends Biochem. Sci. 40, 468-479.

Wang, L.-T., Wang, S.-N., Chiou, S.-S., Liu, K.-Y., Chai, C.-Y., Chiang, C.-M., Huang, S.-K., Yokoyama, K.K., and Hsu, S.-H. (2019). TIP60-dependent acetylation of the SPZ1-TWIST complex promotes epithelial-mesenchymal transition and metastasis in liver cancer. Oncogene 38, 518-532.

Wang, R.-H., Zhao, T., Cui, K., Hu, G., Chen, Q., Chen, W., Wang, X.-W., SotoGutierrez, A., Zhao, K., and Deng, C.-X. (2016). Negative reciprocal regulation between Sirt 1 and Per2 modulates the circadian clock and aging. Sci. Rep. 6, 28633. Welz, P.-S., Zinna, V.M., Symeonidi, A., Koronowski, K.B., Kinouchi, K., Smith, J.G., Guillén, I.M., Castellanos, A., Crainiciuc, G., Prats, N., et al. (2019). BMAL1-Driven Tissue Clocks Respond Independently to Light to Maintain Homeostasis. Cell 177, 1436-1447.e12.

Westermark, P.O. (2016). Linking Core Promoter Classes to Circadian Transcription. PLoS Genet. 12, e1006231.

Wong, J.C.Y., Smyllie, N.J., Banks, G.T., Pothecary, C.A., Barnard, A.R., Maywood, E.S., Jagannath, A., Hughes, S., van der Horst, G.T.J., MacLaren, R.E., et al. (2018). Differential roles for cryptochromes in the mammalian retinal clock. FASEB J. 32, 4302-4314.

Wu, D., and Rastinejad, F. (2017). Structural characterization of mammalian bHLHPAS transcription factors. Curr. Opin. Struct. Biol. 43, 1-9. 
Xiao, Y., Nagai, Y., Deng, G., Ohtani, T., Zhu, Z., Zhou, Z., Zhang, H., Ji, M.Q., Lough, J.W., Samanta, A., et al. (2014). Dynamic interactions between TIP60 and p300 regulate FOXP3 function through a structural switch defined by a single lysine on TIP60. Cell Rep. 7, 1471-1480.

Xu, H., Gustafson, C.L., Sammons, P.J., Khan, S.K., Parsley, N.C., Ramanathan, C., Lee, H.-W., Liu, A.C., and Partch, C.L. (2015). Cryptochrome 1 regulates the circadian clock through dynamic interactions with the BMAL1 C terminus. Nat. Struct. Mol. Biol. 22, 476-484.

Xu, Y., Padiath, Q.S., Shapiro, R.E., Jones, C.R., Wu, S.C., Saigoh, N., Saigoh, K., Ptáček, L.J., and Fu, Y.-H. (2005). Functional consequences of a CKI $\delta$ mutation causing familial advanced sleep phase syndrome. Nature 434, 640-644.

Yang, C., Wu, J., and Zheng, Y.G. (2012). Function of the active site lysine autoacetylation in Tip60 catalysis. PLoS One 7, e32886.

Ye, R., Selby, C.P., Ozturk, N., Annayev, Y., and Sancar, A. (2011). Biochemical analysis of the canonical model for the mammalian circadian clock. J. Biol. Chem. 286, 25891-25902.

Ye, R., Selby, C.P., Chiou, Y.Y., Ozkan-Dagliyan, I., Gaddameedhi, S., and Sancar, A. (2014). Dual modes of CLOCK:BMAL1 inhibition mediated by Cryptochrome and period proteins in the mammalian circadian clock. Genes Dev. 28, 1989-1998.

Yoo, S.-H., Mohawk, J.A., Siepka, S.M., Shan, Y., Huh, S.K., Hong, H.-K., Kornblum, I., Kumar, V., Koike, N., Xu, M., et al. (2013). Competing E3 ubiquitin ligases govern circadian periodicity by degradation of CRY in nucleus and cytoplasm. Cell 152, 10911105 .

Zeng, P.Y., Vakoc, C.R., Chen, Z.C., Blobel, G.A., and Berger, S.L. (2006). In vivo dual cross-linking for identification of indirect DNA-associated proteins by chromatin immunoprecipitation. Biotechniques 41, 694-698.

Zhang, R., Lahens, N.F., Ballance, H.I., Hughes, M.E., and Hogenesch, J.B. (2014). A circadian gene expression atlas in mammals: implications for biology and medicine.

Proc. Natl. Acad. Sci. U. S. A. 111, 16219-16224.

Zhou, H., Gao, J., Lu, Z.Y., Lu, L., Dai, W., and Xu, M. (2007). Role of c-Fos/JunD in protecting stress-induced cell death. Cell Prolif. 40, 431-444.

Zhu, J., Li, C., Gong, C., and Li, X. (2018). Regulation of Pol II Pausing Is Involved in Daily Gene Transcription in the Mouse Liver. J. Biol. Rhythms 33, 350-362.

Zou, Z., Huang, B., Wu, X., Zhang, H., Qi, J., Bradner, J., Nair, S., and Chen, L.-F. (2014). Brd4 maintains constitutively active NF- $\kappa$ B in cancer cells by binding to acetylated RelA. Oncogene 33, 2395-2404. 


\section{Chapter 7: Publication}

Petkau N, Budak H, Zhou X, Oster H, Eichele G. 2019. Acetylation of BMAL1 by TIP60 controls BRD4-P-TEFb recruitment to circadian promoters. Elife 8. 


\section{Acetylation of BMAL1 by TIP60 controls BRD4-P-TEFb recruitment to circadian promoters}

\author{
Nikolai Petkau, Harun Budak ${ }^{\dagger}$, Xunlei Zhou ${ }^{\ddagger}$, Henrik Oster ${ }^{\S}$, Gregor Eichele*
}

Department of Genes and Behavior, Max Planck Institute for Biophysical Chemistry, Göttingen, Germany

Abstract Many physiological processes exhibit circadian rhythms driven by cellular clocks composed of interlinked activating and repressing elements. To investigate temporal regulation in this molecular oscillator, we combined mouse genetic approaches and analyses of interactions of key circadian proteins with each other and with clock gene promoters. We show that transcriptional activators control BRD4-PTEFb recruitment to E-box-containing circadian promoters. During the activating phase of the circadian cycle, the lysine acetyltransferase TIP60 acetylates the transcriptional activator BMAL1 leading to recruitment of BRD4 and the pause release factor $\mathrm{P}-\mathrm{TEFb}$, followed by productive elongation of circadian transcripts. We propose that the control of BRD4-P-TEFb recruitment is a novel temporal checkpoint in the circadian clock cycle.

Present address: ${ }^{\dagger}$ Department of Molecular Biology and Genetics, Science Faculty, Atatürk University, Erzurum, Turkey; Institute of Anatomy and Cell Biology, University of Heidelberg, Heidelberg, Germany; Institute of Neurobiology,Center of Brain, Behavior and Metabolism, University of Lübeck, Lübeck, Germany

Competing interests: The authors declare that no competing interests exist.

Funding: See page 16

Received: 01 November 2018 Accepted: 10 July 2019 Published: 11 July 2019

Reviewing editor: Asifa Akhtar, Max Planck Institute for Immunobiology and Epigenetics, Germany

(a) Copyright Petkau et al. This article is distributed under the article is distib terms of the Creative Commons Attribution License, which permits unrestricted use and redistribution provided that the original author and source are credited.
DOI: https://doi.org/10.7554/eLife.43235.001

\section{Introduction}

Circadian clocks are genetically encoded, self-sustained molecular oscillators that drive biological rhythms with a period close to $24 \mathrm{hr}$ (Partch et al., 2014). They are found in most organisms and even in cultured tissues and cells (Balsalobre et al., 1998; Brown et al., 2012; Yoo et al., 2004). In mammals, these clocks are organized in a network controlled by a master pacemaker in the hypothalamic suprachiasmatic nucleus (SCN) (Dibner et al., 2010). Physiological processes such as energy metabolism, locomotion, hormone secretion, and cognitive functions are subject to circadian regulation (Bass and Takahashi, 2010; Gerstner and Yin, 2010; Panda, 2016). The influence of circadian rhythmicity is highlighted by the fact that in each tissue $~ 10 \%$ of the transcriptome is under the control of the circadian clock (Zhang et al., 2014) and that disruption of circadian rhythms is tied to the development of various diseases including metabolic disorders and cancer (Bedrosian et al., 2016; Musiek and Holtzman, 2016).

The molecular circadian oscillator consists of interlinked positive and negative feedback elements (Reppert and Weaver, 2002). Positive components are the heterodimers of CLOCK (circadian locomotor output cycles kaput) and BMAL1 (brain and muscle ARNT-like protein 1) that bind to E-box promoter elements of Per1-3 (Period), Cry1/2 (Cryptochome) and Rev-ErbA $\alpha / \beta$ (Nr1d1/2; nuclear receptor subfamily 1 , group $D$, members 1 and 2) that encode repressor proteins PER, CRY and NR1D (Takahashi, 2017).

Genome-wide analysis of core circadian regulators revealed temporally restricted chromatin associations of circadian activators and repressors in the course of the circadian day (Koike et al., 2012; Menet et al., 2012; Rey et al., 2011). It is proposed that at the onset of the circadian cycle repressor CRY1 is part of the chromatin-bound CLOCK-BMAL1-complex located near transcriptionally silent RNA polymerase II (Pol II). Upon degradation of CRY1, coactivators such as CBP/p300 are recruited followed by Per and Cry transcription. During the repression phase of the circadian cycle, newly synthesized CRY2 and PER proteins associate with CLOCK-BMAL1, thereby shutting down 
their own transcription (Koike et al., 2012). Recruitment of CRY to the CLOCK-BMAL1 heterodimer is facilitated by acetylation of BMAL1 at lysine 538 by the acetyltransferase activity attributed to CLOCK (Doi et al., 2006; Hirayama et al., 2007). BMAL1 acetylation peaks during the repression phase (Hirayama et al., 2007), although data by Nakakata and co-workers (Nakahata et al., 2008; Nakahata et al., 2009) suggest that the presence of acetylated BMAL1 extends into the activation phase.

An important issue that the above feedback mechanisms leave unresolved concerns the binding of CLOCK-BMAL1 heterodimers to E-box promoter elements that initiates Pol II-mediated transcription (Koike et al., 2012; Stratmann et al., 2012). Well-recognized, rate-limiting steps that regulate transcription are the recruitment of Pol II to promoters and Pol II release from promoter-proximal pause sites (Jonkers and Lis, 2015; Liu et al., 2015). Pause release of Pol II and the transition to productive elongation requires the activity of the P-TEFb (positive transcription elongation factor $\mathrm{b}$ ) complex, composed of T-type cyclins and CDK9 (cyclin-dependent kinase 9) (Jonkers and Lis, 2015; Liu et al., 2015). P-TEFb is recruited to promoters through interaction with specific transcription factors and other proteins like BRD4 (bromodomain-containing protein 4) and SEC (super elongation complex) (Jonkers and Lis, 2015; Liu et al., 2015; Luo et al., 2012; Shi and Vakoc, 2014). After such recruitment, P-TEFb phosphorylates the carboxy-terminal domain (CTD) of Pol II at Ser2 and additionally phosphorylates the pausing factors NELF (negative elongation factor) and DSIF (DRB sensitivity inducing factor). Upon phosphorylation, NELF is evicted from Pol II and DSIF becomes a positive elongation factor. Pol II pausing serves as a checkpoint allowing for rapid and synchronous expression of genes (Boettiger and Levine, 2009; Gilchrist et al., 2012; Lagha et al., 2013; Lin et al., 2011; Liu et al., 2015).

BRD4 is a member of the bromo-domain and extra terminal domain (BET) protein family which bind through their bromo-domains to acetylated lysines of histones and of transcription factors (Shi and Vakoc, 2014). Bound BRD4 recruits P-TEFb to promoter proximal paused Pol II (Jonkers and Lis, 2015; Liu et al., 2015). Small molecule inhibitors that block BRD4 (e.g. JQ1) from binding to acetylated lysines prevent productive elongation (Shi and Vakoc, 2014). Thus BRD4 protein is a key player in the regulation of productive transcription elongation. Therefore, acetyltransferases that acetylate BRD4 binding partners would play an important role in regulating Pol II pause release. The lysine acetyltransferase TIP60 (60 kDa Tat-interactive protein) is recruited to active promoters (Ravens et al., 2015) and plays a role in early steps of transcription elongation in mammals and flies (Kusch et al., 2014; Ravens et al., 2015; Shi et al., 2014). TIP60 is an essential protein (Hu et al., 2009).

Here we provide evidence that TIP60 is a critical component of the circadian clock. During the activation phase of the circadian cycle, TIP60 acetylates BMAL1, which triggers recruitment of the BRD4-P-TEFb complex to E-box-containing circadian promoters leading to Pol II pause release and productive elongation of circadian transcripts. These findings suggest that pause release is a critical regulatory step in the transcription of circadian clock regulated genes and that TIP60 plays a central role in this process.

\section{Results}

Inhibition of CDK9 and BRD4 abolishes circadian oscillations

Synchronized fibroblasts stably expressing the clock-driven luciferase reporter Bmal1-LUC (Nagoshi et al., 2004) show sustained reporter expression with a period of $\sim 26 \mathrm{hr}$. In the presence of flavopiridol (FP), a potent inhibitor of the CDK9 subunit of P-TEFb (Rahl et al., 2010), the amplitude of luciferase activity rhythms was suppressed in a dose dependent manner concomitant with an increased period length until, at high doses, the rhythm was fully lost (Figure 1A; Figure 1-figure supplement 1A). Treatment of fibroblasts with FP also strongly reduced endogenous mRNA expression levels of $\mathrm{Dbp}$ (D-site albumin promoter binding protein), Per1, and $\mathrm{Nr} 1 \mathrm{~d} 1$ (Figure 1-figure supplement 1C) that are direct CLOCK-BMAL1 targets (Stratmann et al., 2012). These findings suggest that circadian clock gene regulation not only occurs at the initiation of transcription (Le Martelot et al., 2012) but also at the level of Pol II pause release and productive elongation. Triazolothienodiazepine (JQ1) is a potent and selective inhibitor of BRD4 (Shi and Vakoc, 2014). JQ1 treatment of Bmal1-LUC reporter fibroblasts dampened luciferase activity rhythms in a dose- 

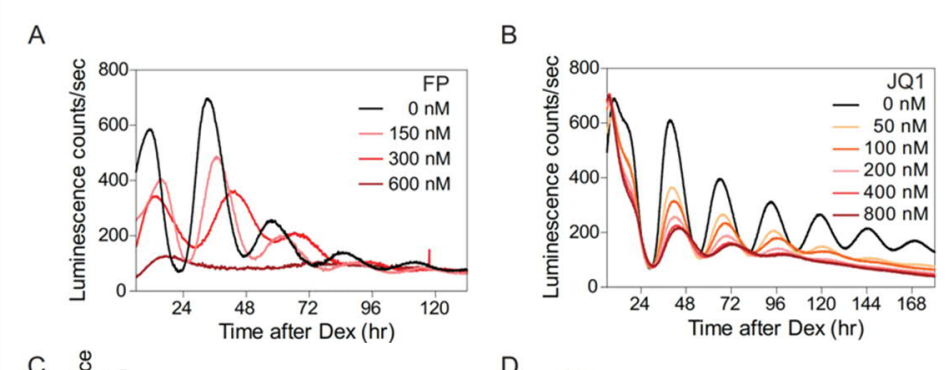
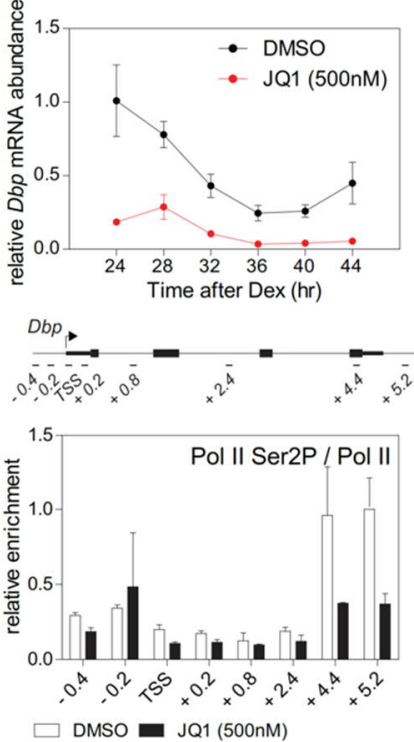

D $b p$
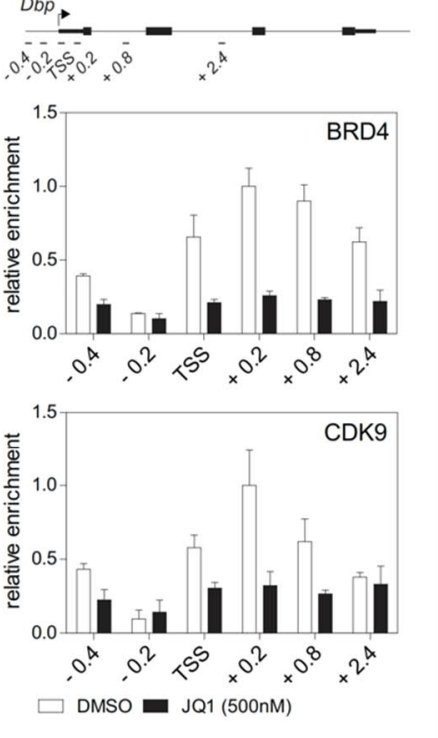

Figure 1. BRD4 controls clock gene expression. (A and B) Bioluminescence recordings of dexamethasone (Dex) synchronized Bmal1-LUC fibroblasts treated with increasing concentrations of flavoperidol (A) or JQ1 (B) $(n=4)$. (C) Dbp mRNA dampening in Dex-synchronized fibroblasts by $500 \mathrm{nM} \mathrm{JO1}(n=3$. two-way ANOVA see Supplementary file 1). (D and E) ChIP analysis of BRD4 and CDK9 (D) and Ser2-phosphorylated Pol II (normalized to total Pol II) (E) for the Dbp gene carried out $24 \mathrm{hr}$ after Dex synchronization of the cells $(n=3)$. Schematic diagrams show the genomic structure of the Dbp locus. Horizontal lines represent PCR-amplified genomic regions. All data are shown as mean \pm SD. Note that Dbp has several additional intronic Pol II pausing sites (Sobel et al., 2017). For validation of ChIP-grade quality of antibodies see Figure 1-figure supplement 2. DOl: https://doi.org/10.7554/eLife.43235.002

The following figure supplements are available for figure 1 :

Figure supplement 1. BRD4 controls expression of E-box-controlled clock genes.

DOl: https://doi.org/10.7554/eLife.43235.003

Figure supplement 2. Validation of antibodies used for ChIP studies either deficient cells or antibody isotype controls were used to validate the ChIP-grade quality of the antisera. DOI: https://doi.org/10.7554/eLife.43235.004 
dependent manner and evoked a lengthening of the period (Figure 1B; Figure 1-figure supplement 1B). JQ1 also resulted in a strong dampening of the rhythm of endogenous $D b p$ expression in these cells (Figure 1C). In the presence of JQ1, enrichment of BRD4 and CDK9 at the transcription start site (TSS) of Dbp was markedly reduced (Figure 1D). Ser2 phosphorylation of Pol II, at the 3'end of Dbp, was strongly diminished (Figure 1E; Figure 1-figure supplement 1D) suggesting that Pol II pause release had been suppressed (Harlen and Churchman, 2017). Reduced Dbp expression could be due to lower levels of BMAL1. To show that this is not the case, we constitutively expressed ectopic CMV promoter-driven Bmal1-myc. BRD4 inhibition by JQ1 strongly reduced endogenous expression levels of Dbp mRNA but had no effect on mRNA or protein levels of Bmal1-myc (Figure 1-figure supplement 1E). Furthermore, BRD4 inhibition neither affected endogenous mRNA nor protein levels of Bmal1 (Figure 1-figure supplement 1F) in Bmal1-LUC fibroblasts at $24 \mathrm{hr}$ after Dex synchronization. Finally, the binding of BMAL1 and of acetylated BMAL1 (ACBMAL1) to the Dbp promoter was not affected by BRD4 inhibition in these cells (Figure 1-figure supplement 1G). Together, our JQ1 inhibitor studies suggest that BRD4-P-TEFb is recruited to the E-box-containing clock-controlled gene Dbp which subsequently allows Pol II pause release and transcription elongation. Thus, for this circadian clock-controlled gene, Pol II pause release may serve as an important regulatory step of its transcription.

\section{Acetylation of BMAL1 at lysine 538 is required to initiate circadian productive transcription elongation}

BRD4 binds to acetylated lysines of transcription factors/histones at the promoter of paused genes (Shi and Vakoc, 2014). The observed strong effect of JQ1 on the cellular circadian rhythm raises the possibility that among BRD4 targets there are core clock components, such as BMAL1, which is acetylated at Lys538 (Hirayama et al., 2007) and could therefore provide a binding site for BRD4. We mutated this particular lysine to an arginine (BMAL1 ${ }^{\mathrm{K} 538 \mathrm{R}}$ ) using the CRISPR/Cas9 technique (Figure 2-figure supplement $1 \mathrm{~A}-1 \mathrm{C}$ ). BMAL1 ${ }^{\mathrm{K} 538 \mathrm{R}}$ protein was as stable as wildtype BMAL1 (Figure 5-figure supplement 1A), and subcellular localization and phosphorylation status of both forms were similar (Hirayama et al., 2007). We also found an unchanged enrichment of both BMAL1 variants at the promoters of the Dbp, Per1, and Nr1d1 genes (Figure 2-figure supplement 1C). Next, the interaction of BRD4 and CDK9 with BMAL1 was examined in control fibroblasts and fibroblasts containing engineered BMAL1 ${ }^{\mathrm{K} 538 R}$. Immunoprecipitation experiments showed that in BMAL1 ${ }^{\mathrm{K} 538 \mathrm{R}}$ expressing cells this interaction was markedly reduced (Figure $2 \mathrm{~A}$ ). When wildtype cells were JQ1-treated, this interaction was substantially dampened (Figure 1-figure supplement $1 \mathrm{H}$ ). ChIP experiments demonstrated that BMAL1 ${ }^{\mathrm{K} 538 \mathrm{R}}$ mutant cells showed a diminished recruitment of BRD4 and of the P-TEFb subunit CDK9 to the TSS of Dbp (Figure 2B), Per1, and Nr1d1 (Figure 2figure supplement 1E). In addition, enrichment of Ser2-phosphorylated Pol II was markedly reduced (Figure 2B; Figure 2-figure supplement 1E; Figure 2-figure supplement 2). Since both, control fibroblasts and BMAL1 ${ }^{\mathrm{K} 538 \mathrm{R}}$ mutant cells stably expressed a clock-driven luciferase reporter, we assessed the effect of the K538R mutation on luminescence rhythms. In BMAL1 ${ }^{\mathrm{K} 538 \mathrm{R}}$ cells virtually no rhythmic luciferase reporter expression was recorded for any of the three clones (Figure $2 \mathrm{C}$ ). In addition, in these cells there was a pronounced reduction of peak expression of endogenous Dbp (Figure 2D), Per1, and Nr1d1 mRNAs (Figure 2-figure supplement 1F). It is possible that BMAL1 acetylation could additionally promote transcription initiation. However, the occupancy of the general transcription factor TFIIE $\alpha$ (part of the Pol II transcription initiation complex; Sainsbury et al., 2015) at the TSS of Dbp, Per1, and Nr1d1 genes was not affected in mutant cells (Figure 2-figure supplement 1G). This favors the idea that transcription elongation, and not initiation, is the process that is primarily regulated by acetylation of BMAL1. Our data support a mechanism in which acetylated BMAL1 recruits BRD4-P and TEFb to E-box-containing circadian clock genes which then results in a release of Pol II from its paused state, thereby allowing productive elongation.

TIP60 is essential for a functional circadian clock in the mouse

BMAL1 acetylation is carried out by CLOCK (Hirayama et al., 2007). However, we found that in CLOCK-deficient fibroblasts BMAL1 was acetylated at Lys 538 to a similar extent as observed in wildtype cells (Figure 3A). CLOCK ${ }^{\text {mutA }}$ fails to acetylate histones H3 and H4 (Doi et al., 2006) and shows greatly diminished activity in the acetylation of BMAL1 (Hirayama et al., 2007). We found that both 


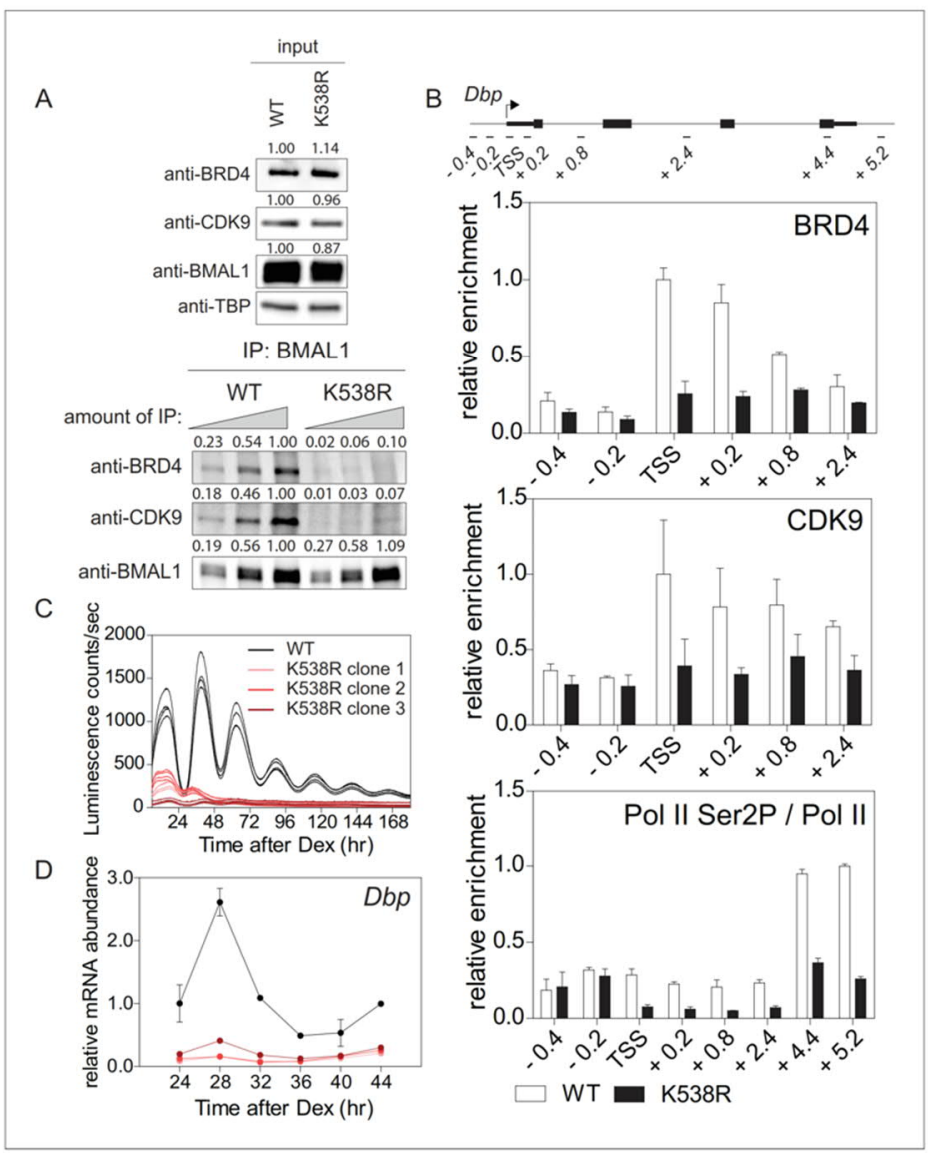

Figure 2. Lys538 acetylation of BMAL1 Is essential for transcription elongation. (A) Interaction of BMAL1 or BMAL1 ${ }^{\text {KS38R }}$ with BRD4 and CDK9 as shown by immunoblotting of nuclear extracts from synchronized fibroblast 24 hr after Dex treatment (two-fold dilutions). TBP (TATA-binding protein) was used as loading control. Numerical values represent intensities of chemiluminescence signals of individual bands, normalized to wildtype and loading control for input samples. (B) ChIP analysis of BRD4, CDK9 and Ser2P-Pol II (normalized to total Pol II) binding to the Dbp gene in wildtype and BMAL1 ${ }^{\mathrm{KS} 38 R}$ synchronized fibroblasts as shown in $(A)(n=3)$. (C) Bmal1-LUC bioluminescence tracings of synchronized wildtype fibroblasts and of three independent BMAL1 ${ }^{K 538 R}$ clones $(n=4)$. (D) Dbp mRNA expression analysis of wildtype or BMAL $1^{K 538 R}$ fibroblasts taken from the experiment shown in (C) $(n=3$, two-way ANOVA, see Supplementary file 1). All data are shown as mean \pm SD. DOI: https://doi.org/10.7554/eLife. 43235.005

The following figure supplements are available for figure 2:

Figure supplement 1. CRISPR/Cas9-mediated generation and characterization of BMAL ${ }^{\mathrm{KS} 38 R}$ mutant cells. DOl: https://doi.org/10.7554/eLife.43235.006

Figure supplement 2. Characterization of BMAL $1{ }^{1538 R}$ mutant cells Pol II and Ser2P-Pol II ChIP analysis of Dex synchronized wildtype or BMAL1 ${ }^{\mathrm{K} 338 R}$ fibroblasts $(n=3)$.

Figure 2 continued on next page 
CLOCK variants showed a minimal increase of BMAL1 acetylation compared to a control (Figure 3B). Importantly, BMAL1 acetylation levels in the presence of CLOCK or CLOCK ${ }^{\text {mutA }}$ were indistinguishable. Together, these data suggest the existence of alternative acetyltransferases that catalyze BMAL1 acetylation. A candidate is TIP60 that co-purifies with CLOCK-BMAL1 (Doi et al., 2006) and acetylates a broad range of transcription factors (Judes et al., 2015). Addition of TIP60, but not enzymatically inactive TIP60 ${ }^{\mathrm{C} 369 \mathrm{~A} ; E 403 \mathrm{O}}$ (Yang et al., 2012), strongly enhanced BMAL1 acetylation (Figure 3B).

This unexpected discovery of a new potential regulator of the circadian clock prompted us to generate conditional TIP60 mice to assess the in-vivo function of this enzyme and provide fibroblasts that we then used to study the molecular clock in vitro. Tip $60^{\mathrm{fl} /-}$ mice carrying one conditional and one deleted allele of Tip60 (Figure 4-figure supplement 1A and B) were mated to Syt10-CRE driver mice. This driver is strongly active in the postmitotic neurons of the SCN (Husse et al., 2011) circumventing cell lethality associated with TIP60-deficiency (Hu et al., 2009). All offspring carrying two copies of the knock-in Cre allele (Syt $10^{\mathrm{Cre} / \mathrm{Cre}} \mathrm{Tip} 6 \mathrm{f}^{\mathrm{fl} / \mathrm{N}}$ ) and half of the mice carrying one copy of the knock-in Cre allele (Syt10 ${ }^{\mathrm{Cre} /+} \mathrm{Tip} 60 \mathrm{fl} /$-) $)$ became immediately arrhythmic upon release into

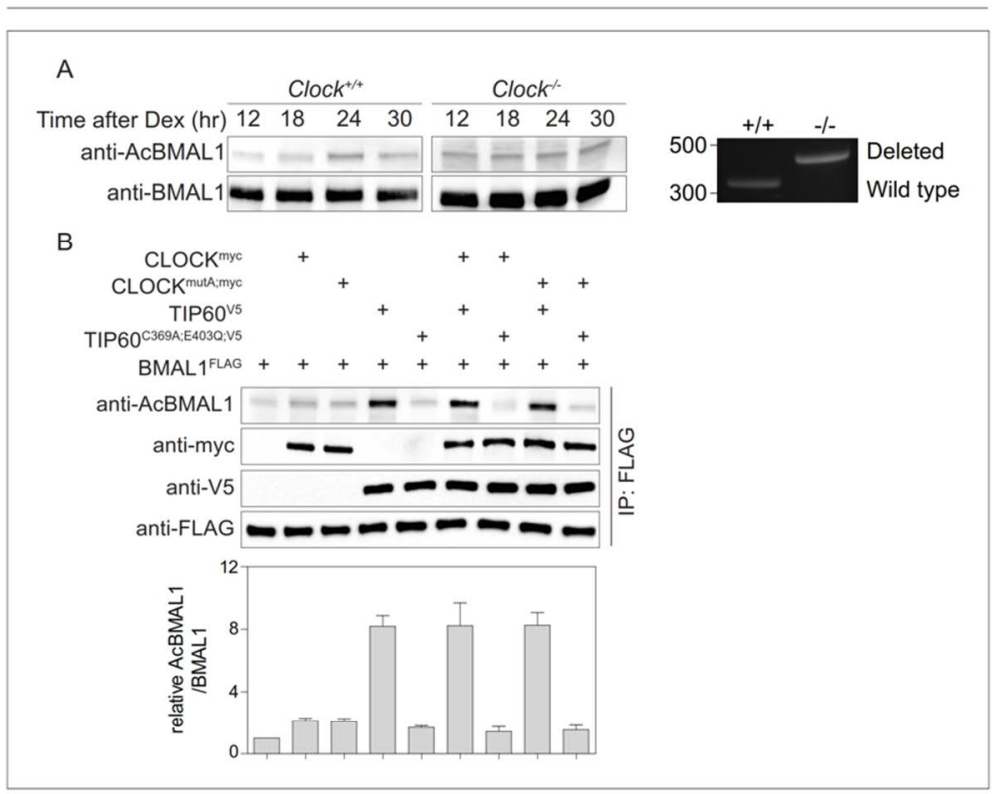

Figure 3. Acetylation of BMAL1 in CLOCK-deficient cells. (A) BMAL1 IP analysis of control and Clock-deficient MEF nuclear extracts over a $24 \mathrm{hr}$ time course. For both genotypes, equivalent amounts of BMAL1 were included as shown in the anti-BMAL1 probed western blot. Lys538 acetylation of BMAL1 was detected using an antiAcBMAL1 antibody (left). Wildtype and mutant cells were PCR-genotyped to confirm their genotype (right) (DebaL1 transiently overexpressed in HEK293T cells in the combinations indicated. Lysates were subjected to IPs and immunoblotted with antibodies indicated $(n=3)$. Data are shown as mean $\pm S D$.

DOI: https://doi.org/10.7554/eLife. 43235.008 


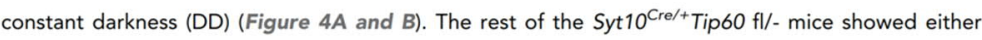
longer $(29 \%)$ or normal $(21 \%)$ free-running periods. Such a Cre dosage dependence was also observed with Syt10-CRE-mediated deletion of Bmal1 $1^{f / f l}$ and, therefore, characterizes this particular driver (Husse et al., 2011). Immunohistochemical staining for TIP60 in the SCN showed nuclear signal, which was absent in arrhythmic Syt $10^{\mathrm{Cre} /+} \mathrm{Tip} 60 \mathrm{fl} /$ - mice (Figure 4C). Moreover, circadian expression patterns of Per1, Dbp, and Bmal1 in the SCN were severely dampened (Figure 4D) in line with the behavioral arrhythmicity observed in these animals. Nuclear staining of SCN sections from Syt $10^{\mathrm{Cre} /+} \mathrm{Tip} 6 \mathrm{O}^{+/+}$and arrhythmic Syt $10^{\mathrm{Cre} /+} \mathrm{Tip} 60 \mathrm{fl} /$ - mice showed no obvious changes in SCN morphology or in the number of SCN cells (control: $827 \pm 60$ cells per section; mutant: $850 \pm 68$ cells per section). In addition, no TUNEL signal, indicating apoptotic cells, could be

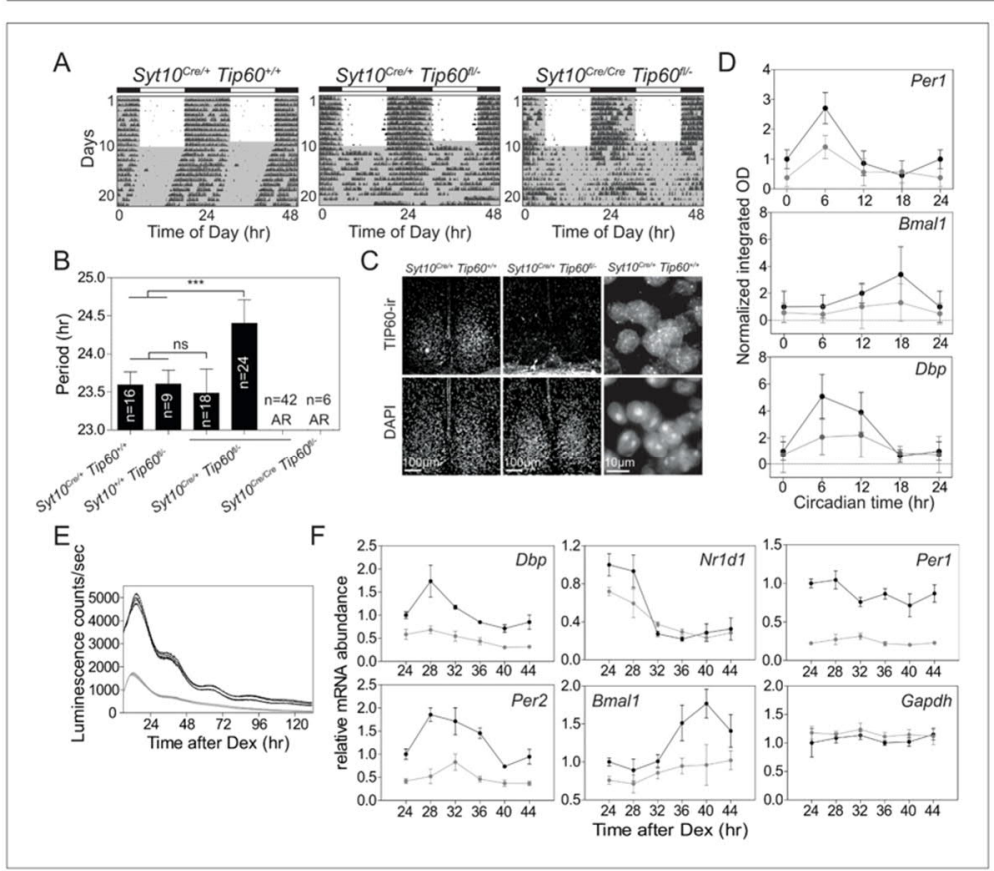

Figure 4. TIP60-deficiency evokes a circadian phenotype in mice and disrupts rhythmic clock gene expression in the SCN and in MEFs. (A) Doubleplotted actograms of control (Syt10 $\left.{ }^{\mathrm{Cre} /+} \mathrm{Tip} 6 \mathrm{O}^{+/+}\right)$and mutant $\left(\mathrm{Syt} 10^{\mathrm{Cre} /+} \mathrm{Tip} 60 \mathrm{fl} /\right.$-, Syt $10^{\mathrm{Cre} / \mathrm{Cre}} \mathrm{Tip} 60^{\mathrm{fl} / /}$ ) mice under $12 \mathrm{hr}: 12 \mathrm{hr}$ light-dark and constant darkness conditions. Gray shadings indicate dark phases. (B) Free-running periods determined by $\chi^{2}$ periodogram analysis. AR: arrhythmic $\left({ }^{\star \star \star} p<0.001\right.$, one-way ANOVA with Bonferroni post-test). (C) TIP60-immunoreactivity (ir) in the SCN of control (Syt10 ${ }^{\mathrm{Cre} /+} \mathrm{Tip}^{\mathrm{T}} \mathrm{O}^{+/ \hbar}$ ) and arrhythmic mutant $\left(\right.$ Syt10 $0^{\mathrm{Cr} / *} \mathrm{Tip} 6 \mathrm{fl} /-$ ) mice. (D) Densitometric quantification of clock gene mRNA expression from radioactive in situ hybridization analysis of control $\left(\right.$ Syt $10^{\mathrm{Cre} /+}$ Tip $60^{+/+}$; black) and mutant (Syt10 $0^{\mathrm{Cre} /+} \mathrm{Tip} 60 \mathrm{fl} /-;$ gray) SCN sections $(\mathrm{n}=3$; two-way ANOVA, see Supplementary file 1). (E)

Bioluminescence recordings of synchronized Tip60 $0^{f /-} ;$ Bmal1-LUC MEFs transduced with AdGFP (black) or AdCre (gray) $(n=4)$. (F) mRNA expression analysis of Dex synchronized Tip60 f// MEFs transduced with either AdGFP (black) or AdCre (gray) ( $n=3$; two-way ANOVA, see Supplementary file 1). All data are shown as mean $\pm \mathrm{SD}$.

DOI: https://doi.org/10.7554/eLife. 43235.009

The following figure supplement is available for figure 4 :

Figure supplement 1. Generation and validation of the Tip60-deficient mice and MEFs derived from such animals. DOI: https://doi.org/10.7554/eLife.43235.010 
detected in the SCN of TIP60-deficient animals (Figure 4-figure supplement 1C). Together, these experiments indicate that $\mathrm{SCN}$ cells were fully viable after deletion of Tip60.

The essential circadian function of TIP60 was also seen in MEFs. Immortalized MEFs were prepared from Tip $60^{f / /}$ mouse embryos and were transduced with a Bmal1-LUC reporter. Confluent, that is non-dividing, MEFs were infected with AdCre to delete TIP60. Cell confluency is sufficient to induce cell cycle quiescence (Hayes et al., 2005), thus bypassing the role of TIP60 in cell cycle regulation (Sykes et al., 2006; Tang et al., 2006). Deletion resulted in a major dampening of the rhythmic expression of the luciferase reporter and a significantly reduced expression of endogenous clock genes (Figure 4E and F), but not of several housekeeping genes (Figure 4F; Figure 4-figure supplement 1D). The deletion of TIP60 in confluent MEFs did not affect cell viability (Figure 4-figure supplement 1E) nor did adenoviral transduction itself alter the circadian rhythm of a luciferase reporter (Figure 4-figure supplement 1F). Thus, abrogation of TIP60 function has major effects on locomotor activity and the rhythmic expression of clock genes in the $\mathrm{SCN}$ in vivo and in postmitotic MEFs.

\section{TIP60 acetylates BMAL1 at lysine 538}

The severe circadian phenotype in the mouse, the strong changes of clock gene expression in TIP60deficient SCN and MEFs, and co-transfection experiments (Figure $3 B$ ) all suggest that BMAL1 could be a direct substrate of TIP60. Co-transfection experiments showed that Lys538 of BMAL1 was acetylated by TIP60 in a dose-dependent manner and that acetyltransferase-deficient TIP $60^{\text {C } 369 A ; E 4030}$ (Yang et al., 2012) led to baseline acetylation levels of BMAL1 similar to mock transfected cells (Figure 3B; Figure 5A). Lys538 appeared to be the predominant lysine acetylated by TIP60 as the signal obtained with an anti-pan-AcK antibody was strongly diminished in cells co-transfected with BMAL1 ${ }^{\mathrm{K} 538 \mathrm{R}}$ and TIP60 (Figure 5A). TIP60 co-precipitated with wildtype and BMAL1 ${ }^{\mathrm{K} 538 \mathrm{R}}$ suggesting an interaction between the two proteins (Figure 5A; Figure 5-figure supplement $1 \mathrm{~A}$ ). Acetylation of BMAL1 did not influence this interaction. Both, BMAL1 and TIP60 ${ }^{\mathrm{V} 5}$ proteins bound to the $E$ boxes of the Dbp gene (Figure 5-figure supplement 1B). Recombinant TIP60 GST acetylated recombinant BMAL1 ${ }^{\text {GST }}$ at Lys538 (Figure 5B) and, in TIP60-deficient MEFs, Lys538 acetylation of endogenous BMAL1 was markedly reduced (Figure $5 C$ ). When endogenous TIP60 was replaced by TIP60 C369A;E403Q in the Bmal1-LUC reporter cell line, luciferase rhythms and mRNA rhythms of endogenous Dbp were abolished (Figure $5 D$ and E). Collectively, these data provide strong evidence that BMAL1 is a substrate of TIP60.

\section{TIP60 controls productive elongation of circadian transcripts}

So far, we have shown that TIP60 acetylates BMAL1 at Lys538 and that abrogation of TIP60 function has major effects on the rhythmic expression of clock genes (Figure 4). BMAL1 ${ }^{\mathrm{K} 538 \mathrm{R}}$ failed to interact with BRD4 and P-TEFb (Figure 2A and B). Furthermore, Pol II Ser2 phosphorylation, an indicator of productive elongation, was markedly reduced in BMAL1 ${ }^{\mathrm{K} 538 \mathrm{R}}$ cells (Figure $2 \mathrm{~B}$, Figure 2-figure supplement 1E; Figure 2-figure supplement 2). It follows that deletion of TIP60 should abrogate BRD4-P-TEFb recruitment and Pol II pause release. Indeed, in confluent TIP60-deficient Dex synchronized fibroblasts, BMAL1 was hypoacetylated (Figures $5 \mathrm{C}$ and $6 \mathrm{~A}$ ) and there was a major loss of interaction between BMAL1 and BRD4 (Figure $6 A$ and $B$ ) as well as between BMAL1 and CDK9 (Figure 6C). ChIP experiments showed that enrichment of BRD4 and CDK9 at the TSS of Dbp, Per1, and $\mathrm{Nr} 1 \mathrm{~d} 1$ genes was strongly reduced and Ser2-phosphorylated Pol II occupancy was diminished (Figure 6D; Figure 6-figure supplement 1A and B).

Above we provided evidence that acetylation of BMAL1 plays a key role in transcription elongation rather than initiation (Figure 2-figure supplement 1G). Since TIP60 acetylates BMAL1, TIP60 deletion should not affect transcription initiation, which was indeed the case. Deletion of Tip60 did not affect TFIIE $\alpha$ recruitment to the TSS of Dbp, Per1, and Nr1d1 genes (Figure 6-figure supplement 1C).

\section{Rhythmicity of productive elongation of circadian transcripts}

Collectively, our experiments provide evidence that TIP60-mediated acetylation of BMAL1 is an essential element of the positive limb of the circadian clock oscillator. TIP60-mediated BMAL1 acetylation leads to the recruitment of BRD4 and of the pause release factor P-TEFb to clock gene TSSs. 


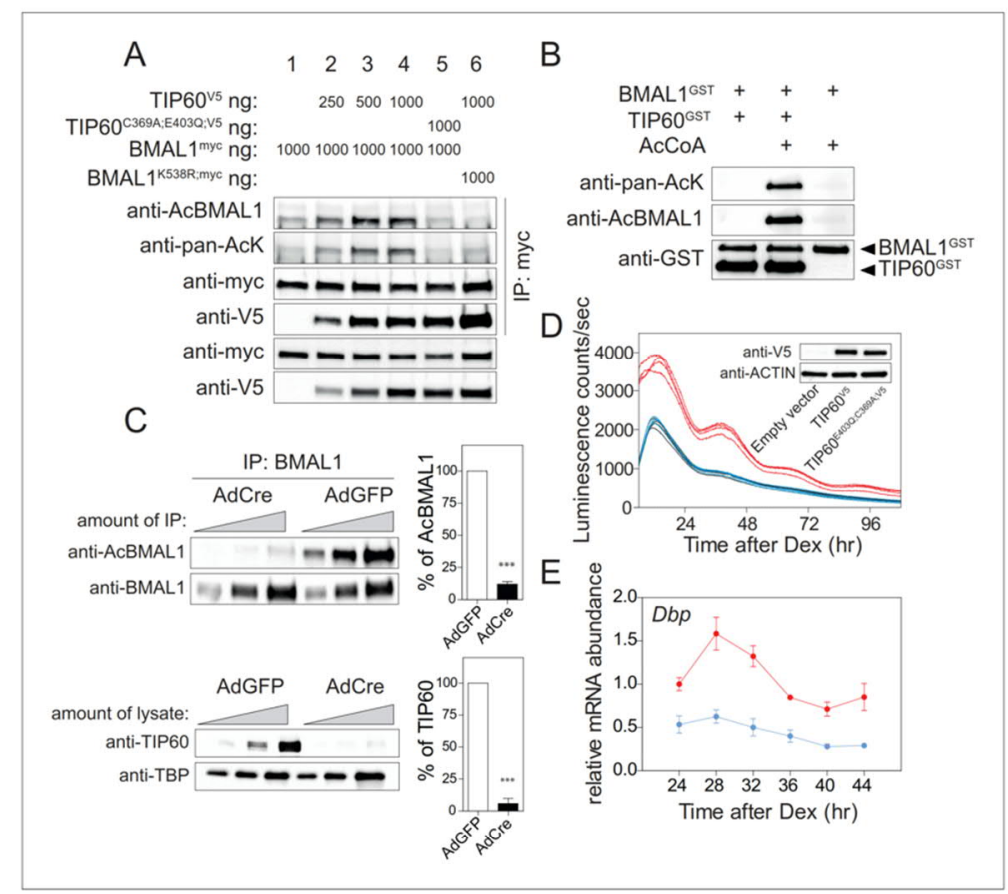

Figure 5. TIP60 acetylates BMAL1. (A) BMAL1 ${ }^{\text {myc }}, B M A L 1^{\mathrm{K} 538 R}, \mathrm{TIP}^{\mathrm{V}}$, and TIP60 ${ }^{\mathrm{C} 369 \mathrm{~A}: \mathrm{E} 403 \mathrm{Q}: \mathrm{V5}}$ were transiently overexpressed in HEK293T cells in the combinations indicated on top. Lysates were subjected to IPs using the antibodies indicated. (B) Recombinant BMAL1 ${ }^{\text {GST }}$, TIP60 ${ }^{\mathrm{GST}}$ and acetyl-COA were incubated and the presence of acetylated BMAL1 was detected by immunoblotting using either a pan-acetyl or a Lys538-specific AcBMAL1 antibody. (C) IPs (top) and nuclear extracts (bottom) from AdGFP (control) and AdCre (mutant) transduced unsynchronized Tip $60^{\sharp / /-}$ MEF (two-fold dilutions) were immunoblotted with antibodies indicated and signal intensities were quantified and normalized. Data are shown as mean of all relative values $\pm S D(n=3)$. (D) Tip $60^{f / /}$;

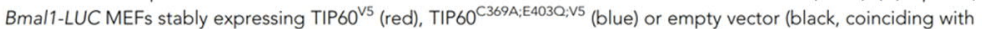
the blue tracing) were transduced with AdCre and bioluminescence was recorded $(n=4)$. (E) Dbp expression profiles in TIP60 ${ }^{\text {V5 }}$ and TIP60 $3669 A_{1}: 4030 ; \mathrm{VS}$ cells shown in (D). ( $n=3$; two-way ANOVA, see Supplementary file 1$)$. DOI: https://doi.org/10.7554/eLife. 43235.011

The following figure supplement is available for figure 5 :

Figure supplement 1. TIP60 interacts with BMAL1 on chromatin.

DOI: https://doi.org/10.7554/eLife.43235.012

This, in turn, allows productive elongation of CLOCK-BMAL1-controlled genes. This positive limb of the circadian cycle is counteracted by a negative feedback. This balance between positive and negative regulation, characteristic for the circadian cycle, should also be seen in temporal occupancy profiles of pause release factors at the promoters of E-box-controlled clock genes. Approximately $24 \mathrm{hr}$ after synchronization of the cells, we observed the strongest enrichment of BMAL1 and of acetylated BMAL1 at the promoters of Dbp, Per1, and Nr1d1 genes (Figure 7A, green). The concentration of acetylated BMAL1 in nuclear extracts also showed its maximum by that time (Figure 7B). In parallel, the occupancy of BRD4 peaked at target gene promoters (Figure 7A) and Ser2 phosphorylation of Pol II showed a maximum (Figure 7A; Figure 7-figure supplement 1). Consequently, the 


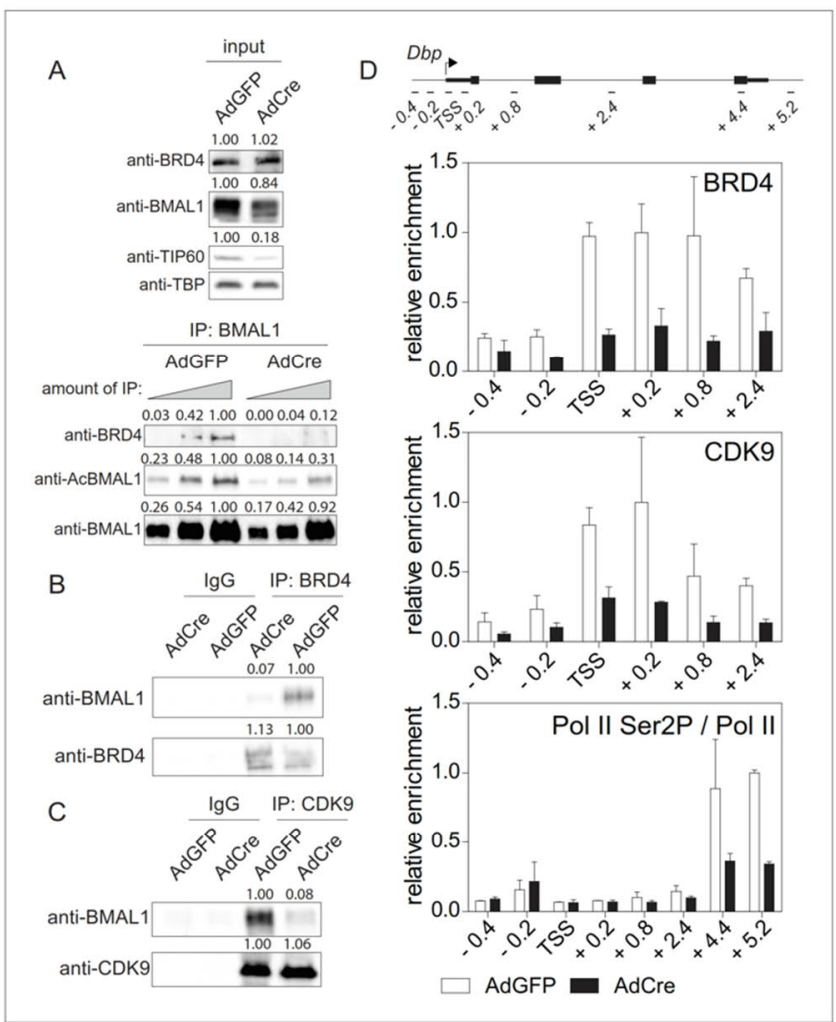

Figure 6. TIP60 controls productive elongation. (A) Interaction of BMAL1 with BRD4 in Tip60 ${ }^{f / /}$ MEFs transduced with either AdGFP (control) or AdCre (mutant). Immunoblots show results with increasing equivalents of IPs (twofold dilutions) from nuclear extracts isolated from cells $24 \mathrm{hr}$ after Dex synchronization. (B) BRD4 or (C) CDK9 IPs from AdGFP or AdCre transduced Tip $60^{\text {th/- }}$ MEF nuclear extracts were immunoblotted with antibodies indicated. Analyses were carried out $24 \mathrm{hr}$ after Dex synchronization. (D) ChIP analysis for BRD4, CDK9 and Ser2P-Pol II (normalized to total Pol II) in the promoter or the $3^{\prime}$-end of Dbp for the cells shown in (A). Data are shown as mean $\pm S D(n=3)$. Numerical values represent intensities of chemiluminescence signals of individual bands, normalized to wildtype and loading control for input samples.

DOI: https://doi.org/10.7554/eLife. 43235.013

The following figure supplement is available for figure 6 :

Figure supplement 1. TIP60 controls productive elongation of circadian genes.

DOl: https://doi.org/10.7554/eLife.43235.014

abundance of Dbp, Per1, and Nr1d1 mRNA peaked between 24 and 28 hr (Figure 7A). Taken together, our data show that TIP60-dependent BRD4 recruitment, Pol II pause release, and productive elongation are precisely timed over the circadian cycle and in this way exert a temporal control over the circadian clock oscillator. 
eLIFE

Chromosomes and Gene Expression | Neuroscience

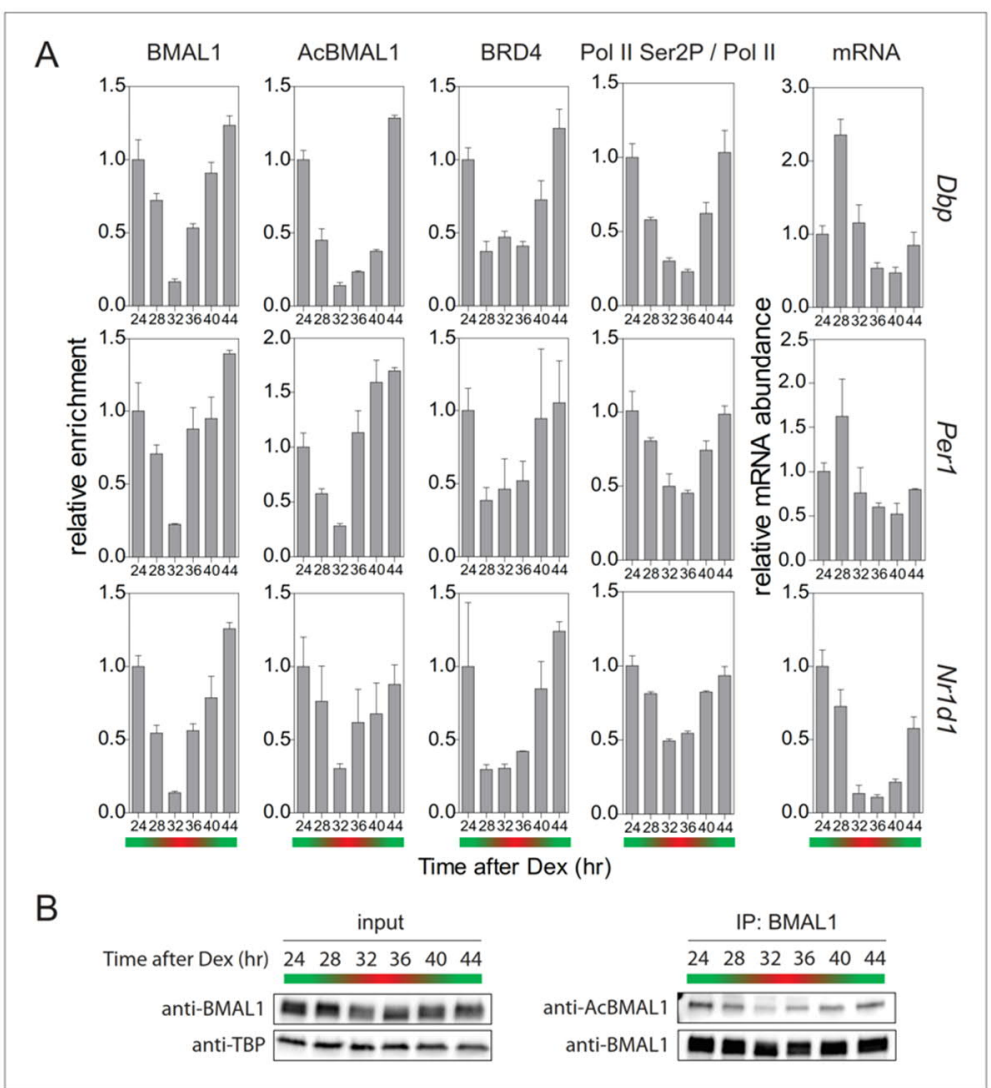

Figure 7. Rhythmic profile of productive elongation. (A) ChIP profiles of Dex-synchronized MEFs over a course of $24 \mathrm{hr}$. ChIP shows time-of-daydependent enrichment of BMAL1, acetylated BMAL1, BRD4, and Ser2P-Pol II (normalized to total Pol II) at the promoter or 3'-end (Ser2P-Pol II) of Dbp, Per1, and Nr1d1 genes. The rightmost column reveals time-dependence of mRNA accumulation for these genes. Data are shown as mean $\pm \operatorname{SD}(n=3)$, (B) Immunoblot analysis with the indicated antibodies of Dex-synchronized fibroblast nuclear extracts and BMAL1 IPs. The color bars represent the activation (green) and repression (red) phases of the circadian cycle. DOI: https://doi.org/10.7554/elife.43235.015

The following figure supplement is available for figure 7 :

Figure supplement 1. Rhythmic profile of Pol II and Ser2P-Pol II abundance ChIP profiles of Dex-synchronized MEFs over a course of $24 \mathrm{hr}$. DOl: https://doi.org/10.7554/eLife.43235.016

\section{Discussion}

Genome-wide ChIP studies (Koike et al., 2012; Menet et al., 2012; Rey et al., 2011) show that at the onset of the circadian cycle, the circadian repressor protein CRY1 is associated with chromatinbound CLOCK-BMAL1 located near Pol II. In this complex Pol II is bound to the clock gene promoters but is not competent to drive RNA synthesis. At this time in the circadian cycle Pol II is phosphorylated at Ser5 (Koike et al., 2012), which is indicative for promoter-proximal pausing and 
recruitment of the mRNA capping complex to Pol II (Harlen and Churchman, 2017). Evidence for Pol II pausing to occur at this time at circadian promoters can also be found in global run-on experiments measuring transcriptionally engaged Pol II during the circadian cycle (Fang et al., 2014). Strong enrichment of Pol II at the TSS of the Nr1d1 gene was observed at the onset of the circadian cycle, suggesting that Pol II is in a paused state. Genome-wide and circadian time-resolved quantitative analysis of Pol II abundance at TSSs and gene bodies show a peak of Pol II binding at TSSs, but not the gene bodies of clock genes, at the onset of the circadian cycle (Zhu et al., 2018). A circadian control of promoter proximal pausing and also of Pol II pause release would provide mechanisms capable to accurately time circadian transcription (Jonkers and Lis, 2015; Liu et al., 2015).

In the present work, we have addressed the mechanism of the circadian regulation of Pol II pause release with a focus on proteins that are known to mediate this process. We propose that pause release involves a TIP60-mediated on-chromatin acetylation of Lys538 of BMAL1. BMAL1 acetylation allows the recruitment of the co-activator BRD4 to AcBMAL1. BRD4, in turn, recruits the pause release factor P-TEFb, whose kinase subunit CDK9 then phosphorylates Ser2 of Pol II leading to a release of Pol II from the paused state enabling productive elongation of $E$-box-containing circadian genes (Figure 7A). In TIP60-deficient cells, Lys538 acetylation of BMAL1 was strongly diminished, BRD4-P-TEFb recruitment was reduced, and the expression of Dbp, Per1, and Nr1d1 genes was dampened. If acetylation of BMAL at Lys538 was prevented by a Lys to Arg substitution (BMAL $1^{\mathrm{K} 538 R}$ ), this mutant protein still interacted with TIP60, but recruitment of BRD4 and P-TEFb to the complex was reduced and Pol II pause release and productive elongation were diminished. The dysregulation of the circadian rhythm of locomotor activity in the mouse and perturbation of circadian gene expression in the SCN and in MEFs provide strong genetic evidence for a key role of TIP60 in the circadian clock. Our studies of TIP60-deficient MEFs suggest that rhythmic control of Pol II pause release may be a main process in which TIP60 is involved. Our studies thus implicate Pol II pause release as an additional regulatory step that contributes to the temporal control of the mammalian circadian clock. However, we cannot completely rule out the possibility that the loss of TIP60 could affect post-initiation event other than Pol II pause release and thereby control RNA processing or RNA stability (Core and Adelman, 2019). Kinetic analysis of all steps of the transcription cycle could address this issue.

TIP60 is evolutionarily highly conserved and is involved in the regulation of a series of cellular processes (see Introduction). Thereby, TIP60 has the role of an enzymatically active cofactor in larger multiprotein complexes (Jacquet et al., 2016; Judes et al., 2015). Our studies suggest a similar function of TIP60 within the circadian clock.

Our studies are based on three robustly rhythmic and clock phase-specific genes that harbor multiple CLOCK-BMAL1 binding sites such as the tandem E-box motif (E1-E2) (Paquet et al., 2008; Rey et al., 2011). There are many other strongly rhythmic, phase specific CLOCK-BMAL1-target genes that also harbor multiple E-boxes (Rey et al., 2011; Shimomura et al., 2013). This suggests that regulation by Pol II pause release could extend to the entire class of E-box controlled clock genes. These genes are synchronously expressed and would benefit from a precisely timed Pol II pause release process. It is not clear, however, whether BRD4-P-TEFb mediated Pol II pause release applies for other types of clock-controlled genes. Temporal analyses of Pol II pause release at the transcriptome-wide scale can address these issues.

We found that in fibroblasts, but also in the SCN, deletion of TIP60 resulted in a nearly complete loss of rhythmic expression of numerous circadian genes suggesting that in these tissues and for these genes TIP60 function is non-redundant with CBP/p300 acetyltransferase that also binds to circadian promoters. The strongest argument for BMAL1 being a substrate of TIP60 is that recombinant TIP60 directly acetylates recombinant BMAL1. Although p300 can acetylate BMAL1 it does so at lysine 500 (Weinert et al., 2018). The abundance of BRD4 at clock gene promoters was drastically reduced in BMAL1 ${ }^{\mathrm{K} 338 \mathrm{R}}$ cells, but it is likely that there are other acetylated substrates, such as histones H3 and H4 (Etchegaray et al., 2003; Koike et al., 2012; Ripperger and Schibler, 2006; Vollmers et al., 2012), to which BRD4 could also be recruited and affect the circadian clock.

It is proposed that CLOCK acetylates its heterodimeric partner BMAL1 at lysine 538 . This leads to recruitment of CRY protein and, thus, initiates the repression phase of the circadian cycle (Hirayama et al., 2007). We found that TIP60-mediated acetylation of BMAL1 occurs during the activation phase of the cycle, which is consistent with the data of Nakahata and co-workers (Nakahata et al., 2008; Nakahata et al., 2009). Our finding of strong acetylation of Lys538 of 
BMAL1 in CLOCK-deficient fibroblasts and the absence of an effect on the BMAL1 acetylation status in a reaction with a catalytically inactive CLOCK mutant protein raises some doubt about the efficiency of CLOCK as an acetyltransferase for BMAL1. It is noteworthy that CLOCK acetylates argininosuccinate synthase in the cytosol of U2OS cells (Lin et al., 2017). Since our experiments aimed at transcriptional regulation of the clock, they were carried out with nuclear extracts, they do not address cytosolic acetyltransferase activity of CLOCK.

TIP60 acetylates BMAL1 during the activation phase, but it is not clear by which mechanism BMAL1 is deacetylated during the repression phase. One possibility is a SIRT1-dependent deacetylation of BMAL1 (Nakahata et al., 2008). Alternatively, recruitment of histone deacetylases by PER to the CLOCK-BMAL1 heterodimer (Duong et al., 2011; Kim et al., 2014) would lead to deacetylation of BMAL1. Another possibility is that promoter-associated acetylated BMAL1 could be subject to rapid proteolytic turnover (Stratmann et al., 2012) obviating the need for a specific deacetylase.

Taken together, our study provides evidence that the positive limb of the circadian clock requires TIP60-mediated acetylation of BMAL1, at least for the genes studied here. This could allow a precise and synchronous expression of E-box-containing clock genes and in this way also contribute to robustness and precision of the circadian clock. Robust and synchronous expression are the hallmarks of genes involved in stimulus-controlled pathways, whose transcription is also often regulated through Pol II pause release (Jonkers and Lis, 2015; Liu et al., 2015; Sawarkar et al., 2012; Siegal and Rushlow, 2012).

\section{Materials and methods}

\section{Tip60 targeting vector and generation of experimental animals}

Bacteria (Strain EL350) and plasmids (pL451 and pL452) used for recombineering were obtained from the $\mathrm{NCl}$ (Biological Resources Branch). A $15.7 \mathrm{~kb}$ fragment of Tip60 genomic DNA was cloned from a BAC clone (bMQ-331N14, Sanger Institute) into a targeting vector carrying a Pol2-DTA cassette for negative selection. The first loxP site was inserted $637 \mathrm{bp}$ upstream of the Tip60 ATG. The second loxP site together with a FRT-flanked PGK-neo cassette was inserted 3,065 bp downstream of the ATG. The 5' end of the homologous arm of the targeting vector was $4.5 \mathrm{~kb}$ and the $3^{\prime}$ end of the homologous arm was $7.6 \mathrm{~kb}$. ES cell targeting and generation of Tip $60^{\mathrm{fl} / \mathrm{t}}$ founder mice was commissioned to PolyGene Transgenics (Switzerland). The FRT-flanked PGK-neo selection cassette was removed by crossing mice with a Flippase-expressing deleter line. Primers P1-P3 used for PCR genotyping of the Tip60 mice are listed in Supplementary file 2.

To generate animals for locomotor activity recording, Tip $60^{f / / f l}$ mice were crossed to a Syt $10 \mathrm{Cre}$ driver mouse line resulting in Cre-mediated deletion of TIP60 predominately in the SCN (Husse et al., 2011). To generate Tip60 $0^{f /-}$ mice for subsequent MEF isolation, Tip $60^{f / f l}$ mice were crossed to a ubiquitous CMVCre driver mouse line to generate $\mathrm{Tip} 6 \mathrm{O}^{+/} \pm$which were then used for breeding with Tip $60^{f / / f l}$ mice to obtain Tip $60^{f / /}$ offspring. For both types of studies, mice were previously backcrossed to a C57BL/6 background for at least 10 generations. Mouse handling was carried out in accordance with the German Law on Animal Welfare and was ethically approved and licensed by the Office of Consumer Protection and Food Safety of the State of Lower Saxony (license numbers 33.11.42502-04/072/07 and 33.9-42502-04-12/0719).

\section{Generation of Bmal1 ${ }^{K 538 R}$ mutant cells}

$B$ mal1 ${ }^{K 538}$ mutant cell lines were generated using CRISPR/Cas9-based genome editing. Fibroblasts stably expressing a clock-driven luciferase reporter (Bmal1-LUC) (Nagoshi et al., 2004) were cotransfected with an all-in-one plasmid pSpCas9(BB)-2A-Puro (PX459) harboring CAS9, puromycinresistance gene, and guide RNA (sgRNA), and a single-stranded oligodeoxynucleotide (ssODNs) template comprising the point mutation. The sgRNA was designed using the online tool (http:// crispr.mit.edu/). The target sequence of sgRNA was 5'-GGTCCTCCGTTCTTCCATTCGTA-3' (PAM sequence is underlined). Transfected cells were selected with puromycin $(1 \mu \mathrm{g} / \mathrm{ml})$ for two days and clonal cell lines were isolated by limiting dilution. Genomic DNA of these clones was extracted and subjected to sequencing. Primers used for screening are listed in Supplementary file 2. 
Plasmids

Bmal1, Tip60 ORFs were amplified by PCR and cloned into pcDNA3.1 vector containing either $\mathrm{C}$-terminal myc- or V5-tags. Single site mutations were introduced by using the QuikChange II Sitedirected Mutagenesis Kit (Agilent Technologies). Tip60 $0^{V 5}$ and Tip60 C369A:E403Q:V5 were cloned into pLenti PGK Hygro DEST vector. Bmal1-FLAG was from M.J. Rossner (LMU Munich). Myc-Clock and myc-Clock ${ }^{\text {mutA }}$ were from P. Sassone-Corsi. Lentiviral Bmal1-dLuc reporters were from S.A. Kay (USC Los Angeles), pBABE-puro SV40 LT (\#13970), pCL-Eco (\#12371), pMD2.G (\#12259), psPAX2 (\# 12260), pLenti PGK Hygro DEST (\#19066), and pSpCas9(BB)-2A-Puro (\# 48139) plasmids were from Addgene.

\section{RNA analysis by quantitative Real-Time PCR (qPCR)}

Total RNA was extracted using RNeasy Plus Mini Kit (Qiagen). cDNA was synthesized using Maxima $\mathrm{H}$ Minus First Strand cDNA Synthesis Kit (Thermo Fisher Scientific). Real-time PCR reactions were performed on a CFX96 Real-Time PCR Detection System (Bio-Rad) with IO SYBR Green Supermix (Bio-Rad). Eef1a or Gapdh expression was used for normalization. Primers were as described (Oster et al., 2006) except for Gapdh, TIP60, 18S, Actb, and 36b4 (Supplementary file 2).

\section{Radioactive in situ Hybridization}

Mice were entrained for about 2 weeks to a $12 \mathrm{hr}: 12 \mathrm{hr}$ light dark cycle and subsequently released into constant darkness. On the second day after lights-off mice were sacrificed at four time points and brains were collected and frozen in O.C.T. medium (Tissue-Tek). Templates for Dbp, Per1, and Bmal1 were as described (Oster et al., 2003; Oster et al., 2006). In situ hybridization with ${ }^{35} \mathrm{~S}$ labeled antisense RNA probes was carried out on frozen sections through the SCN. Relative quantification of expression strength was performed by densitometric analysis of autoradiograph films.

\section{Immunohistochemistry}

Cryo-sections through the SCN were fixed with $4 \%$ paraformaldehyde for $15 \mathrm{~min}$ at $4^{\circ} \mathrm{C}$, boiled several times in $10 \mathrm{mM}$ sodium citrate $(\mathrm{pH}$ 6.4) for $5 \mathrm{~min}$, and permeabilized for $15 \mathrm{~min}$ with $0.5 \%$ Triton-X-100. Sections were blocked with $10 \%$ FBS in PBS followed by incubation with anti-TIP60 antibody and a fluorescent secondary antibody. Nuclei were stained with DAPI.

\section{Cell culture}

Tip60 ${ }^{f / /}$ MEFs were isolated following standard procedures. MEFs, fibroblasts (NIH-3T3 Bmal1-LUC; Nagoshi et al., 2004), and HEK293T cells were cultured and passaged in standard medium (DMEM, high glucose, GlutaMAX supplement, pyruvate; $10 \%$ fetal bovine serum; $100 \mathrm{U} / \mathrm{ml}$ penicillin and 100 $\mu \mathrm{g} / \mathrm{ml}$ streptomycin [all Thermo Fisher Scientific]) at $37^{\circ} \mathrm{C}$ in a humidified incubator with $5 \% \mathrm{CO}_{2}$. MEFs and fibroblasts were synchronized by treatment with $100 \mathrm{nM}$ dexamethasone, a synthetic glucocorticoid that induces expression of Per1 (Reddy et al., 2009; Reddy et al., 2012). JQ1 (500 nM), Flavopiridol $(300 \mathrm{nM})$ or vehicle (final $0.05 \%$ DMSO) were added to standard medium after synchronization. All cells were tested using morphology and PCR-based approaches to confirm their identity. Cell lines were tested negative for mycoplasma (Sigma-Aldrich).

\section{Transfection and viral transduction}

HEK293T cells were transiently transfected using $1 \mathrm{mg} / \mathrm{ml}$ polyethylenimine solution (Sigma-Aldrich). After two days, cells were used for western blotting and immunoprecipitations (IPs). Tip $60^{\mathrm{f} / \mathrm{M}} \mathrm{MEFs}$ were immortalized with SV4O LT retrovirus. Immortalized MEFs were grown to confluence and transduced with AdGFP or AdCre adenoviruses (Vector Development Laboratory, Baylor College of Medicine, Houston, TX, USA) in standard medium containing $3 \%$ fetal bovine serum (low-serum) and 8 $\mu \mathrm{g} / \mathrm{ml}$ polybrene (Sigma-Aldrich). After two days, medium was changed to low-serum medium and cells were cultured for another 4-6 days. For lentiviral transduction (luciferase experiments) viruses were produced (Ramezani and Hawley, 2002) and concentrated using Lenti-X Concentrator (Clontech). 


\section{Preparation of cell and nuclear extracts}

Transiently transfected HEK293T cells were lysed in $50 \mathrm{mM}$ Tris- $\mathrm{HCl}, \mathrm{pH} 8.0 ; 150 \mathrm{mM} \mathrm{NaCl} ; 5 \mathrm{mM}$ EDTA; $15 \mathrm{mM} \mathrm{MgCl}$; $1 \%$ NP40; $1 \mathrm{mM}$ DTT; $10 \mathrm{mM} \mathrm{NaF} ; 10 \mathrm{mM}$ NAM; Complete EDTA-free (Roche) for $30 \mathrm{~min}$ on ice and lysate was cleared by centrifugation $\left(20,000 \times \mathrm{g}, 4^{\circ} \mathrm{C}, 10 \mathrm{~min}\right)$. Samples were boiled in SDS sample buffer or used for IP. Anti-Myc agarose beads (Sigma-Aldrich) were used to precipitate myc-tagged proteins. The precipitates were washed three times with lysis buffer and bound proteins eluted by denaturation in SDS sample buffer.

Nuclear extracts were prepared as described (Dimauro et al., 2012). Nuclei were lysed with 15 passages through an 18-gauge needle, sonicated using Bioruptor ( $30 \mathrm{~s}$ ON/30 s OFF) and cleared by centrifugation $\left(9,000 \mathrm{~g}, 4^{\circ} \mathrm{C}, 30 \mathrm{~min}\right)$. For western blotting samples were denatured in SDS sample buffer. For IPs samples were diluted 3-fold in dilution buffer (20 mM HEPES pH 7.9; $1.5 \mathrm{mM} \mathrm{MgCl}$; 0.2 mM EDTA; 20\% glycerol; $10 \mathrm{mM}$ NAM; Complete EDTA-free [Roche]) and incubated for $2 \mathrm{hr}$ at $4^{\circ} \mathrm{C}$ on a rotating wheel. Antibody was added and samples were put back on the rotating wheel. The next day, antibody-protein complexes were collected by Dynabead Protein $\mathrm{G}$ Beads (Thermo Fisher Scientific) and washed three times with wash buffer (20 mM HEPES pH 7.9; $1.5 \mathrm{mM} \mathrm{MgCl} ; 166 \mathrm{mM}$ $\mathrm{NaCl} ; 0.2$ mM EDTA; 20\% glycerol; 0,33\% Triton-X-100; 10 mM NAM; Complete EDTA-free [Roche]). Proteins were eluted by boiling in SDS sample buffer and analyzed by western blotting. For acetylated BMAL1, western blotting was first performed with ACBMAL1 antibody followed by a harsh stripping (Abcam) step and subsequent reaction with the pan BMAL1 antibody. Signal intensities were determined on an ImageQuant LAS4010 imager (General Electric) and quantified using ImageQuant software.

\section{Chromatin immunoprecipitation (ChIP)}

For BMAL1 (NB100-2288), acetylated BMAL1 (AB15396), BRD4 (A301-985A), CDK9 (sc-484 or sc13130), TFIIE $\alpha$ (ab28177), Pol II (MABI0601), and Ser2 phosphorylated Pol II (04-1571) ChIP analysis were performed as described (Lin et al., 2012) with minor modifications. Fibroblasts were crosslinked with $1 \%$ formaldehyde for $10 \mathrm{~min}$ at room temperature. The reaction was stopped by adding $125 \mathrm{mM}$ glycine and incubating for $5 \mathrm{~min}$. Cells were washed twice with PBS, scraped and frozen in liquid nitrogen. Dynabead Protein G Beads (Thermo Fisher Scientific) were blocked with $0.5 \%$ BSA $(w / v)$ in PBS and bound to the indicated antibodies. Crosslinked cells were lysed in lysis buffer one and washed with lysis buffer 2. Pellets were resuspended and sonicated in lysis buffer 3 (Bioruptor: $30 \mathrm{~s}$ ON/30 s OFF) to obtain chromatin fragments of 150-600 bp length for promoter analysis and $100-300$ bp for TSS analysis to allow a higher resolution. Sonicated lysates were cleared and incubated overnight at $4^{\circ} \mathrm{C}$ with beads bound with antibody for indicated factors. Beads were washed twice (CDK9, TFIIE, and Pol II Ser2P) or three times (BMAL1, AcBMAL1, Pol II, and BRD4) with lysis buffer 3 , once with lysis buffer 3 with $500 \mathrm{mM} \mathrm{NaCl}$, one time with $\mathrm{LiCl}$ wash buffer and one time with TE plus $50 \mathrm{mM} \mathrm{NaCl}$. DNA was eluted in elution buffer. The eluted complexes were de-crosslinked overnight and RNA and protein were digested using RNase A and Proteinase K. Immunoprecipitated DNA fragments were purified by phenol chloroform extraction and ethanol precipitation and used for GPCR analysis.

For TIP60 ${ }^{\sqrt{5}}$ ChIP assays were performed as described (Adli and Bernstein, 2011; Nelson et al., 2006) with minor modifications. For dual cross-linking Tip $60^{-1 /}$; Tip $60^{v 5} \mathrm{MEFs}$ were fixed with 1.5 $\mathrm{mM}$ ethylene glycol bis(succinimidylsuccinate) (EGS) (Pierce) for $30 \mathrm{~min}$ followed by $1 \%$ formaldehyde for another $15 \mathrm{~min}$. After quenching the reaction by adding glycine to a final concentration of $125 \mathrm{mM}$, cells were lysed in SDS lysis buffer on ice for $10 \mathrm{~min}$, with brief vortexing. Samples were diluted 10-fold in ChIP dilution buffer and sonicated (Bioruptor; $30 \mathrm{~s}$ ON/30 s OFF) to obtain chromatin fragments of 150-600 bp length. V5 (R96025) antibody was added to the chromatin solution and incubated overnight at $4^{\circ} \mathrm{C}$ on a rotating wheel. Immunoprecipitated chromatin complexes (Dynabead Protein G Beads) were washed twice with ice cold low-salt wash buffer, twice with ice cold lithium chloride wash buffer, and twice with ice cold TE buffer. $100 \mu 10 \%$ Chelex 100 Slurry (Bio Rad) was added directly to the washed beads, briefly vortexed, and boiled for 10 min. Input DNA samples were ethanol precipitated and washed with $70 \%$ ethanol. DNA pellets were dissolved in $100 \mu \mathrm{l} 10 \%$ Chelex 100 suspension and boiled for $10 \mathrm{~min}$. Samples were Proteinase K treated and boiled for $10 \mathrm{~min}$. Supernatant was transferred to a new tube and used for qPCR analysis. 
Primer pair that targets an unbound region was used as internal control (Duong et al., 2011) and fold enrichment was calculated using the $2(-\Delta \Delta C T)$ method. Experiments were performed with biological independent triplicates and data normalized to the mean value obtained from the locus with the strongest enrichment. Primer sequences for ChIP-qPCR are listed in Supplementary file 1.

\section{Bioluminescence recording}

After synchronization of cells with dexamethasone medium was changed to recording medium (DMEM with high glucose, without glutamine and phenol red; 2 mM GlutaMAX; $10 \mathrm{mM} \mathrm{HEPES;} \%$ FBS; $100 \mathrm{U} / \mathrm{ml}$ penicillin and $100 \mu \mathrm{g} / \mathrm{ml}$ streptomycin [all Thermo Fisher Scientific] and $0.1 \mathrm{mM}$ D-luciferin [Synchem]). Petri dishes were sealed with transparent plastic film and bioluminescence was recorded in a LumiCycle luminometer (Actimetrics).

For JQ1 and flavopiridol dose dependence experiments, NIH-3T3 Bmal1-LUC fibroblasts were plated on $35 \mathrm{~mm}$ dishes and cultured for 2 days to reach confluency. Cells were synchronized and medium was replaced with the recording medium containing various concentrations of JQ1, flavopiridol or vehicle (final $0.08 \%$ DMSO). For calculation of period, luminescence intensity, and damping rate parameters the curve fitting program LumiCycle (Actimetrics) was used and data obtained from the first day were excluded from analysis because of transient luminescence changes upon the medium change.

\section{In vitro acetylation assay}

Recombinant TIP60 (SignalChem) and BMAL1 (Abnova) proteins were mixed in acetyltransferase assay buffer ( $50 \mathrm{mM}$ Tris- $\mathrm{HCl}$, pH 8.0; $0.1 \mathrm{mM}$ EDTA; $0.4 \mathrm{mM}$ DTT; $25 \mathrm{ng} / \mu \mathrm{l}$ BSA; $5 \%$ glycerol) in the presence or absence of $10 \mu \mathrm{M}$ acetyl-CoA and incubated for $1 \mathrm{hr}$ at $30^{\circ} \mathrm{C}$. The reaction was stopped by denaturation in SDS sample buffer and proteins were analyzed by western blotting.

\section{Flow cytometry}

Cells were stained using the SYTOX AADvanced Dead Cell Stain Kit (Thermo Fisher Scientific) according to the manufacturer's instructions and samples were analyzed using the Accuri C6 Flow Cytometer (BD). To provide a positive control cells were boiled for $3 \mathrm{~min}$.

\section{Acknowledgements}

We thank Dr. JW Lough and Dr. B Amati for antibodies, Dr. MP Antoch for Clock ${ }^{-1}$ and Bmal1 $1-$ MEFs and Dr. SA Kay, Dr. P Sassone-Corsi and MJ Rossner for sharing vectors.

\section{Additional information}

\begin{tabular}{lll}
$\begin{array}{l}\text { Funding } \\
\text { Funder }\end{array}$ & Grant reference number & Author \\
\hline Volkswagen Foundation & Lichtenberg Fellowship & Henrik Oster \\
\hline Max-Planck-Gesellschaft & Open-access funding & Gregor Eichele \\
\hline
\end{tabular}

The funders had no role in study design, data collection and interpretation, or the decision to submit the work for publication.

Author contributions

Nikolai Petkau, Conceptualization, Data curation, Validation, Investigation, Visualization, Methodology, Writing-original draft, Writing-review and editing, Designed and supervised research, Prepared the manuscript with input from all authors; Harun Budak, Generated mouse model, Performed behavior analysis; Xunlei Zhou, Methodology, Generated mouse model; Henrik Oster, Data curation, Validation, Investigation, Methodology, Writing-original draft, Writingreview and editing; Gregor Eichele, Conceptualization, Resources, Supervision, Funding acquisition, Validation, Writing-original draft, Project administration, Writing-review and editing, Design and supervise research, Prepared the manuscript with input from all authors 
Author ORCIDs

Nikolai Petkau (i) https://orcid.org/0000-0001-9168-3473

Harun Budak (iD) https://orcid.org/0000-0002-7371-8959

Xunlei Zhou (i] https://orcid.org/0000-0002-9635-7068

Henrik Oster (i] https://orcid.org/0000-0002-1414-7068

Gregor Eichele (i] https://orcid.org/0000-0002-2863-9127

Ethics

Animal experimentation: Mouse handling was carried out in accordance with the German Law on Animal Welfare and was ethically approved and licensed by the Office of Consumer Protection and Food Safety of the State of Lower Saxony (license numbers 33.11.42502-04/072/07 and 33.9-4250204-12/0719).

Decision letter and Author response

Decision letter https://doi.org/10.7554/eLife. 43235.022

Author response https://doi.org/10.7554/eLife.43235.023

\section{Additional files}

Supplementary files

- Supplementary file 1. Two-way ANOVA statistical analysis.

DOl: https://doi.org/10.7554/elife.43235.017

- Supplementary file 2. Primer sequences.

DOI: https://doi.org/10.7554/eLife.43235.018

- Supplementary file 3. Key resources table.

DOI: https://doi.org/10.7554/eLife.43235.019

- Transparent reporting form

DOl: https://doi.org/10.7554/elife.43235.020

Data availability

All data generated or analysed during this study are included in the manuscript and supporting files.

\section{References}

Adli M, Bernstein BE. 2011. Whole-genome chromatin profiling from limited numbers of cells using nano-ChIP. seq. Nature Protocols 6:1656-1668. DOI: https://doi.org/10.1038/nprot.2011.402, PMID: 21959244 Balsalobre A, Damiola F, Schibler U. 1998. A serum shock induces circadian gene expression in mammalian tissue culture cells. Cell 93:929-937. DOI: https://doi.org/10.1016/S0092-8674(00)81199-X

Bass J, Takahashi JS. 2010. Circadian integration of metabolism and energetics. Science 330:1349-1354. DOI: https://doi.org/10.1126/science.1195027, PMID: 21127246

Bedrosian TA, Fonken LK, Nelson RJ. 2016. Endocrine effects of circadian disruption. Annual Review of Physiology 78:109-131. DOI: https://doi.org/10.1146/annurev-physiol-021115-105102, PMID: 26208951 Boettiger AN, Levine M. 2009. Synchronous and stochastic patterns of gene activation in the Drosophila Boettiger AN, Levine M. 2009. Synchronous and stochastic patterns of gene activation in the Drosop
embryo. Science 325:471-473. DOI: https://doi.org/10.1126/science.1173976, PMID: 19628867

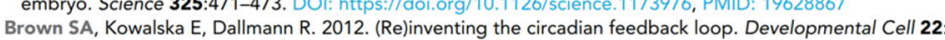
Brown SA, Kowalska E, Dallmann R. 2012. (Re)inventing the circadian feedback loop.
477-487. DOI: https://doi.org/10.1016/j.devcel.2012.02.007, PMID: 22421040

Core L, Adelman K. 2019. Promoter-proximal pausing of RNA polymerase II: a nexus of gene regulation. Genes Core L, Adelman K. 2019. Promoter-proximal pausing of RNA polymerase II: a nexus
\& Development. DOI: https://doi.org/10.1101/gad.325142.119, PMID: 31123063

\& Development. DOl: https://doi.org/10.1101/gad. 325142.119, PMID: 31123063
Debruyne JP, Noton E, Lambert CM, Maywood ES, Weaver DR, Reppert SM. 2006. A clock shock: mouse Debruyne JP, Noton E, Lambert CM, Maywood ES, Weaver DR, Reppert SM. 2006. A clock shock: mouse
CLOCK is not required for circadian oscillator function. Neuron 50:465-477. DOI: https://doi.org/10.1016/j. neuron.2006.03.041, PMID: 16675400

Dibner C, Schibler U, Albrecht U. 2010. The mammalian circadian timing system: organization and coordination of central and peripheral clocks. Annual Review of Physiology 72:517-549. DOI: https://doi.org/10.1146/ annurev-physiol-021909-135821, PMID: 20148687 
Dimauro I, Pearson T, Caporossi D, Jackson MJ. 2012. A simple protocol for the subcellular fractionation of skeletal muscle cells and tissue. BMC Research Notes 5:513. DOI: https://doi.org/10.1186/1756-0500-5-513, PMID: 22994964

Doi M, Hirayama J, Sassone-Corsi P. 2006. Circadian regulator CLOCK is a histone acetyltransferase. Cell 125: 497-508. DOI: https://doi.org/10.1016/j.cell.2006.03.033, PMID: 16678094

Duong HA, Robles MS, Knutti D, Weitz CJ. 2011. A molecular mechanism for circadian clock negative feedback. Science 332:1436-1439. DOI: https://doi.org/10.1126/science.1196766, PMID: 21680841

Etchegaray JP, Lee C, Wade PA, Reppert SM. 2003. Rhythmic histone acetylation underlies transcription in the mammalian circadian clock. Nature 421:177-182. DOI: https://doi.org/10.1038/nature01314, PMID: 12483227 Fang B, Everett LJ, Jager J, Briggs E, Armour SM, Feng D, Roy A, Gerhart-Hines Z, Sun Z, Lazar MA. 2014. Circadian enhancers coordinate multiple phases of rhythmic gene transcription in vivo. Cell 159:1140-1152. DOI: https://doi.org/10.1016/j.cell.2014.10.022, PMID: 25416951

Gerstner JR, Yin JC. 2010. Circadian rhythms and memory formation. Nature Reviews Neuroscience 11:577-588 DOI: https://doi.org/10.1038/nrn2881, PMID: 20648063

Gilchrist DA, Fromm G, dos Santos G, Pham LN, McDaniel IE, Burkholder A, Fargo DC, Adelman K. 2012. Regulating the regulators: the pervasive effects of pol II pausing on stimulus-responsive gene networks. Genes \& Development 26:933-944. DOI: https://doi.org/10.1101/gad 1877811112 PMID: 22549956

Harlen KM Churchman LS. 2017. The code and beyond: transcription regulation by the RNA polymerase II carboxy-terminal domain. Nature Reviews Molecular Cell Biology 18:263-273. DOl: https://doi.org/10.1038/ carboxy-terminal domain. Nature

Hayes O, Ramos B, Rodríguez LL, Aguilar A, Badia T, Castro FO. 2005. Cell confluency is as efficient as serum starvation for inducing arrest in the $\mathrm{G} 0 / \mathrm{G} 1$ phase of the cell cycle in granulosa and fibroblast cells of cattle. Animal Reproduction Science 87:181-192. DOI: https://doi.org/10.1016/j.anireprosci.2004.11.011, PMID: 15 911169

Hirayama J, Sahar S, Grimaldi B, Tamaru T, Takamatsu K, Nakahata Y, Sassone-Corsi P. 2007. CLOCK-mediated acetylation of BMAL1 controls circadian function. Nature 450:1086-1090. DOI: https://doi.org/10.1038, nature06394, PMID: 18075593

Hu Y, Fisher JB, Koprowski S, McAllister D, Kim MS, Lough J. 2009. Homozygous disruption of the Tip60 gene causes early embryonic lethality. Developmental Dynamics : An Official Publication of the American Association of Anatomists 238:2912-2921. DOl: https://doi.org/10.1002/dvdy.22110, PMID: 19842187

Husse J, Zhou X, Shostak A, Oster H, Eichele G. 2011. Synaptotagmin10-Cre, a driver to disrupt clock genes in the SCN. Journal of Biological Rhythms 26:379-389. DOI: https://doi.org/10.1177/0748730411415363, PMID: 21921292

Jacquet K, Fradet-Turcotte A, Avvakumov N, Lambert JP, Roques C, Pandita RK, Paquet E, Herst P, Gingras AC, Pandita TK, Legube G, Doyon Y, Durocher D, Côté J. 2016. The TIP60 complex regulates bivalent chromatin recognition by $53 \mathrm{bp} 1$ through direct H4K20 me binding and H2AK15 acetylation. Molecular Cell 62:409-421. recognition by 53bp1 through direct H4K20me binding and H2AK15

Jonkers I, Lis JT. 2015. Getting up to speed with transcription elongation by RNA polymerase II. Nature Reviews Jonkers I, Lis JT. 2015. Getting up to speed with transcription elongation by RNA polymerase
Molecular Cell Biology 16:167-177. DOI: https://doi.org/10.1038/nrm3953, PMID: 25693130

Judes G, Rifaï K, Ngollo M, Daures M, Bignon YJ, Penault-Llorca F, Bernard-Gallon D. 2015. A bivalent role of Judes G, Rifaï K, Ngollo M, Daures M, Bignon YJ, Penault-Llorca F, Bernard-Gallon D. 2015. A bivalent role of
TIP60 histone acetyl transferase in human Cancer. Epigenomics 7:1351-1363. DOI: https://doi. org/10.2217/epi.

Kim JY, Kwak PB, Weitz CJ. 2014. Specificity in circadian clock feedback from targeted reconstitution of the NuRD corepressor. Molecular Cell 56:738-748. DOI: https://doi.org/10.1016/j.molcel.2014.10.017, PMID: 25453762

Koike N, Yoo SH, Huang HC, Kumar V, Lee C, Kim TK, Takahashi JS. 2012. Transcriptional architecture and chromatin landscape of the core circadian clock in mammals. Science 338:349-354. DOI: https://doi.org/10. 1126/science.1226339, PMID: 22936566

Kusch T, Mei A, Nguyen C. 2014. Histone H3 lysine 4 trimethylation regulates cotranscriptional H2A variant exchange by Tip60 complexes to maximize gene expression. PNAS 111:4850-4855. DOl: https://doi.org/10. 1073/pnas.1320337111, PMID: 24639513

Lagha M, Bothma JP, Esposito E, Ng S, Stefanik L, Tsui C, Johnston J, Chen K, Gilmour DS, Zeitlinger J, Levine MS. 2013. Paused pol Il coordinates tissue morphogenesis in the Drosophila embryo. Cell 153:976-987. DOI: https://doi.org/10.1016/j.cell.2013.04.045, PMID: 23706736

Le Martelot G, Canella D, Symul L, Migliavacca E, Gilardi F, Liechti R, Martin O, Harshman K, Delorenzi M, Desvergne B, Herr W, Deplancke B, Schibler U, Rougemont J, Guex N, Hernandez N, Naef F, Cyclix Consortium. 2012. Genome-wide RNA polymerase II profiles and RNA accumulation reveal kinetics of Consortium.
transcription and associated epigenetic changes during diurnal cycles. PLOS Biology 10:e1001442. transcription and associated epigenetic changes during diurnal cycles,

DOI: https://doi.org/10.1371/journal.pbio.1001442, PMID: 23209382
Lin C, Garrett AS, De Kumar B, Smith ER, Gogol M, Seidel C, Krumlauf R, Shilatifard A. 2011. Dynamic transcriptional events in embryonic stem cells mediated by the super elongation complex (SEC). Genes \& Development 25:1486-1498. DOI: https://doi.org/10.1101/gad.2059211, PMID: 21764852

Lin CY, Lovén J, Rahl PB, Paranal RM, Burge CB, Bradner JE, Lee TI, Young RA. 2012. Transcriptional amplification in tumor cells with elevated c-Myc. Cell 151:56-67. DOl: https://doi.org/10.1016/j.cell.2012.08. 026, PMID: 23021215 
Lin R, Mo Y, Zha H, Qu Z, Xie P, Zhu ZJ, Xu Y, Xiong Y, Guan KL. 2017. CLOCK acetylates ASS1 to drive circadian Lin R, Mo Y, Zha H, Qu Z, Xie P, Zhu ZJ, Xu Y, Xiong Y, Guan KL. 2017. CLOCK acetylates ASS1 to drive circadian
rhythm of ureagenesis. Molecular Cell 68:198-209. DOl: https://doi.org/10.1016/j.molcel.2017.09.008, PMID: 2 3985504

Liu X, Kraus WL, Bai X. 2015. Ready, pause, go: regulation of RNA polymerase II pausing and release by cellular signaling pathways. Trends in Biochemical Sciences 40:516-525. DOI: https://doi.org/10.1016/j. tibs.2015.07. 03, PMID: 2625422

Luo Z, Lin C, Shilatifard A. 2012. The super elongation complex (SEC) family in transcriptional control. Nature Reviews Molecular Cell Biology 13:543-547. DOI: https://doi.org/10.1038/nrm3417, PMID: 22895430 Menet JS, Rodriguez J, Abruzzi KC, Rosbash M. 2012. Nascent-Seq reveals novel features of mouse circadian transcriptional regulation. eLife 1:e00011. DOI: https://doi.org/10.7554/eLife.00011, PMID: 23150795 Musiek ES, Holtzman DM. 2016. Mechanisms linking circadian clocks, sleep, and neurodegeneration. Science 354:1004-1008. DOI: https://doi.org/10.1126/science.aah4968, PMID: 27885006

Nagoshi E, Saini C, Bauer C, Laroche T, Naef F, Schibler U. 2004. Circadian gene expression in individual fibroblasts: cell-autonomous and self-sustained oscillators pass time to daughter cells. Cell 119:693-705. DOl: https://doi.org/10.1016/i.cell.2004.11.015, PMID: 15550250

Nakahata Y, Kaluzova M, Grimaldi B, Sahar S, Hirayama J, Chen D, Guarente LP, Sassone-Corsi P. 2008. The NAD+-dependent deacetylase SIRT1 modulates CLOCK-mediated chromatin remodeling and circadian control. Cell 134:329-340. DOI: https://doi.org/10.1016/icell. 2008.07.002, PMID: 18662547

Cell $134.329-340$. Nakahata Y, Sahar S, Astarita G, Kaluzova M, Sassone-Corsi P. 2009. Circadian control of the NAD+ salvage pathway by CLOCK-SIRT1. Science 324:654-657. DOI: https://doi.org/10.1126/science.1170803, PMID: 192

Nelson JD, Denisenko O, Bomsztyk K. 2006. Protocol for the fast chromatin immunoprecipitation (ChIP) method. Nature Protocols 1:179-185. DOI: https://doi.org/10.1038/nprot.2006.27, PMID: 17406230

Oster H, Baeriswyl S, Van Der Horst GT, Albrecht U. 2003. Loss of circadian rhythmicity in aging mPer1-1mCry2-/- mutant mice. Genes \& Development 17:1366-1379. DOl: https://doi.org/10.1101/gad.256103, PMID: 12782655

Oster H, Damerow S, Kiessling S, Jakubcakova V, Abraham D, Tian J, Hoffmann MW, Eichele G. 2006. The circadian rhythm of glucocorticoids is regulated by a gating mechanism residing in the adrenal cortical clock. Cell Metabolism 4:163-173. DOI: https://doi.org/10.1016/j.cmet.2006.07.002, PMID: 16890544 Panda S. 2016. Circadian physiology of metabolism. Science 354:1008-1015. DOl: https://doi.org/10.1126/ science.aah4967, PMID: 27885007

Paquet ER, Rey G, Naef F. 2008. Modeling an evolutionary conserved circadian cis-element. PLOS Computational Biology 4:e38. DOI: https://doi.org/10.1371/journal.pcbi.0040038, PMID: 18282089

Partch CL, Green CB, Takahashi JS. 2014. Molecular architecture of the mammalian circadian clock. Trends in Cell Biology 24:90-99. DOI: https:///doi.org/10.1016/j.tcb.2013.07.002, PMID: 23916625

Rahl PB, Lin CY, Seila AC, Flynn RA, McCuine S, Burge CB, Sharp PA, Young RA, 2010, c-Myc regulates

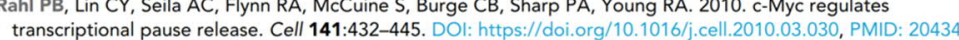
984

Ramezani A, Hawley RG. 2002. Generation of HIV-1-based lentiviral vector particles. Current Protocols in Ramezani A, Hawley RG. 2002. Generation of HIV-1-based lentiviral vector particles. Current Protoco
Molecular Biology 16. DOI: https://doi.org/10.1002/0471142727.mb1622s60, PMID: 18265303

Ravens S, Yu C, Ye T, Stierle M, Tora L. 2015. Tip60 complex binds to active pol II promoters and a subset of enhancers and co-regulates the c-Myc network in mouse embryonic stem cells. Epigenetics \& Chromatin 8:45 DOI: https://doi.org/10.1186/s13072-015-0039-z, PMID: 2655003

Reddy TE, Pauli F, Sprouse RO, Neff NF, Newberry KM, Garabedian MJ, Myers RM. 2009. Genomic determination of the glucocorticoid response reveals unexpected mechanisms of gene regulation. Genom Research 19:2163-2171. DOI: https://doi.org/10.1101/gr.097022.109, PMID: 19801529

Reddy TE, Gertz J, Crawford GE, Garabedian MJ, Myers RM. 2012. The hypersensitive glucocorticoid response specifically regulates period 1 and expression of circadian genes. Molecular and Cellular Biology 32:3756-3767. DOl: https://doi.org/10.1128/MCB.00062-12, PMID: 22801371

Reppert SM, Weaver DR. 2002. Coordination of circadian timing in mammals. Nature 418:935-941. DOI: https:// doi.org/10.1038/nature00965, PMID: 12198538

Rey G, Cesbron F, Rougemont J, Reinke H, Brunner M, Naef F. 2011. Genome-wide and phase-specific DNAbinding rhythms of BMAL1 control circadian output functions in mouse liver. PLOS Biology 9:e1000595. DOI: https://doi.org/10.1371/journal.pbio.1000595, PMID: 21364973

Ripperger JA, Schibler U. 2006. Rhythrnic CLOCK-BMAL1 binding to multiple E-box motifs drives circadian dbp Ripperger JA, Schibler U. 2006. Rhythrnic CLOCK-BMAL1 binding to multiple E-box motifs drives circadian db
transcription and chromatin transitions. Nature Genetics 38:369-374. DOl: https://doi.org/10.1038/ng1738, transcription and
PMID: 16474407

Sainsbury S, Bernecky C, Cramer P. 2015. Structural basis of transcription initiation by RNA polymerase II. Nature Reviews Molecular Cell Biology 16:129-143. DOI: https://doi.org/10.1038/nrm3952, PMID: 25693126 Sawarkar R, Sievers C, Paro R. 2012. Hsp90 globally targets paused RNA polymerase to regulate gene expression in response to environmental stimuli. Cell 149:807-818. DOl: https://doi.org/10.1016/j.cell.2012.02. 61, PMID: 22579285

Shi J, Wang Y, Zeng L, Wu Y, Deng J, Zhang $Q$, Lin Y, Li J, Kang T, Tao M, Rusinova E, Zhang G, Wang C, Zhu H, Yao J, Zeng YX, Evers BM, Zhou MM, Zhou BP. 2014. Disrupting the interaction of BRD4 with diacetylated twis suppresses tumorigenesis in basal-like breast Cancer. Cancer Cell 25:210-225. DOI: https://doi.org/10.1016/1. ccr.2014.01.028, PMID: 24525235 
Shi J, Vakoc CR. 2014. The mechanisms behind the therapeutic activity of BET bromodomain inhibition. Molecular Cell 54:728-736. DOl: https://doi.org/10.1016/j.molcel.2014.05.016, PMID: 24905006

Shimomura K, Kumar V, Koike N, Kim TK, Chong J, Buhr ED, Whiteley AR, Low SS, Omura C, Fenner D, Owens JR, Richards M, Yoo SH, Hong HK, Vitaterna MH, Bass J, Pletcher MT, Wiltshire T, Hogenesch J, Lowrey PL, et al. 2013. Usf1, a suppressor of the circadian clock mutant, reveals the nature of the DNA-binding of the CLOCK:bmal1 complex in mice. elife 2:e00426. DOI: https://doi.org/10.7554/eLife.00426, PMID: 23580255 Siegal ML, Rushlow C. 2012. Pausing on the path to robustness. Developmental Cell 22:905-906. DOI: https:// doi.org/10.1016/j.devcel.2012.04.020, PMID: 22595664

Sobel JA, Krier I, Andersin T, Raghav S, Canella D, Gilardi F, Kalantzi AS, Rey G, Weger B, Gachon F, Dal Peraro M, Hernandez N, Schibler U, Deplancke B, Naef F, CycliX consortium. 2017. Transcriptional regulatory logic of the diurnal cycle in the mouse liver. PLOS Biology 15:e2001069. DOI: https://doi.org/10.1371/journal.pbio. 2001069, PMID: 28414715

Stratmann M, Suter DM, Molina N, Naef F, Schibler U. 2012. Circadian dbp transcription relies on highly dynamic BMAL1-CLOCK interaction with E boxes and requires the proteasome. Molecular Cell 48.277-287. DOl: https://doi.org/10.1016/j.molcel.2012.08.012, PMID: 22981862

Sykes SM, Mellert HS, Holbert MA, Li K, Marmorstein R, Lane WS, McMahon SB. 2006. Acetylation of the p53 DNA-binding domain regulates apoptosis induction. Molecular Cell 24:841-851. DOI: https://doi.org/10.1016/ DNA-binding domain regulates apoptosis

Takahashi JS. 2017. Transcriptional architecture of the mammalian circadian clock. Nature Reviews Genetics 18 164-179. DOI: https://doi.org/10.1038/nrg.2016.150, PMID: 27990019

Tang Y, Luo J, Zhang W, Gu W. 2006. Tip60-dependent acetylation of $\mathrm{p} 53$ modulates the decision between cellcycle arrest and apoptosis. Molecular Cell 24:827-839. DOl: https://doi.org/10.1016/j.molcel.2006.11.021,

PMID: 17189186
Vollmers C, Schmitz RJ, Nathanson J, Yeo G, Ecker JR, Panda S. 2012. Circadian oscillations of protein-coding and regulatory RNAs in a highly dynamic mammalian liver epigenome. Cell Metabolism 16:833-845. DOI: https://doi.org/10.1016/j.cmet.2012.11.004, PMID: 23217262

Weinert BT, Narita T, Satpathy S, Srinivasan B, Hansen BK, Schölz C, Hamilton WB, Zucconi BE, Wang WW, Liu WR, Brickman JM, Kesicki EA, Lai A, Bromberg KD, Cole PA, Choudhary C. 2018. Time-Resolved analysis reveals rapid dynamics and broad scope of the CBP/p300 acetylome. Cell 174:231-244. DOI: https://doi.org/ 10.1016/j.cell.2018.04.033, PMID: 29804834

Yang C, Wu J, Zheng YG. 2012. Function of the active site lysine autoacetylation in Tip60 catalysis. PLOS ONE 7: e32886. DOI: https://doi.org/10.1371/journal.pone.0032886, PMID: 22470428

Yoo SH, Yamazaki S, Lowrey PL, Shimomura K, Ko CH, Buhr ED, Siepka SM, Hong HK, Oh WJ, Yoo OJ, Menaker M, Takahashi JS. 2004. PERIOD2::LUCIFERASE real-time reporting of circadian dynamics reveals persistent circadian oscillations in mouse peripheral tissues. PNAS 101:5339-5346. DOl: https://doi.org/10.1073/pnas circadian oscillations in mouse per
0308709101, PMID: 14963227

Zhang R, Lahens NF, Ballance HI, Hughes ME, Hogenesch JB. 2014. A circadian gene expression atlas in mammals: implications for biology and medicine. PNAS 111:16219-16224. DOl: https://doi.org/10.1073/pnas. 1408886111, PMID: 25349387

Zhu J, Li C, Gong C, Li X. 2018. Regulation of pol II pausing is involved in daily gene transcription in the mouse liver. Journal of Biological Rhythms 33:350-362. DOI: https://doi.org/10.1177/0748730418779526, PMID: 2 9845885 


\section{Chapter 8: Acknowledgments}

First, I would like thank my supervisor, Gregor Eichele, for his continuous support and advice during my $\mathrm{PhD}$. I appreciate the liberty with which I was allowed to work on this project and also his numerous ideas, suggestions and constructive criticisms. I am also grateful to my thesis committee member Henning Urlaub for his suggestions.

Sincere acknowledgements to the members of the extended examination board Prof. Ahmed Mansouri, Prof. André Fiala, Prof. Detlef Doenecke and Dr. Alex Faesen for accepting my invitation to join the committee.

Many thanks to all current and former members of the Gene and Behavior department for the collaborative and friendly working environment. Special thanks to Lars for his friendship and support especially in challenging times, to Martin, Anton, Vinodh, Inga, Insa, and Volodymyr for all the good memories and to Helena for her amicable support. I thank Henrik Oster for many valuable suggestions and his help. I also want to thank Stefanie for the organizational support.

I thank the animal care taker Ute Kunze for looking after the mice and Dirk Reuter and Stefanie Asper for help with genotyping. Thanks to Maren Brockmeyer and Frauke Grabbe for the brain sectioning.

I am very grateful to Tahereh, who supports me in everything I do and makes my life better, easier, and funnier.

Finally, I would like to thank my family for their unconditional support within all these years. 
